\title{
Aspects of the magnetosphere-stellar wind interaction of close-in extrasolar planets
}

Von der Fakultät für Physik und Geowissenschaften der Technischen Universität Carolo-Wilhelmina zu Braunschweig zur Erlangung des Grades eines Doktors der Naturwissenschaften

(Dr.rer.nat.) genehmigte Dissertation

von Jean-Mathias Grießmeier aus Kulmbach 


\section{Bibliografische Information Der Deutschen Bibliothek}

Die Deutsche Bibliothek verzeichnet diese Publikation in der Deutschen Nationalbibliografie; detaillierte bibliografische Daten sind im Internet über http: //dnb. ddb. de abrufbar.

1. Referent: Prof. Dr. rer. nat. U. Motschmann

2. Referent: Univ.-Prof. Mag. Dr. H. O. Rucker eingereicht am: 19. Dezember 2005

mündliche Prüfung (Disputation) am: 16. Februar 2006

Copyright (C) Copernicus GmbH 2006

ISBN 3-936586-49-7

Copernicus GmbH, Katlenburg-Lindau

Druck: Schaltungsdienst Lange, Berlin

Printed in Germany 


\section{Veröffentlichungen von Teilen der Arbeit}

Teilergebnisse aus dieser Arbeit wurden mit Genehmigung der Fakultät für Physik und Geowissenschaften, vertreten durch die Mentorin oder den Mentor der Arbeit, in folgenden Beiträgen vorab veröffentlicht:

Publikationen:

1. H. Lammer, H. I. M. Lichtenegger, Yu. N. Kulikov, J.-M. Grießmeier, N. Terada, N. V. Erkaev, H. K. Biernat, M. L. Khodachenko, I. Ribas, T. Penz, F. Selsis: $C M E$ activity of low mass $M$ stars as an important factor for the habitability of terrestrial exoplanets. Part II: CME induced ion pick up of Earth-like exoplanets in close-in habitable zones, Astrobiology, eingereicht (2006).

2. M. L. Khodachenko, I. Ribas, H. Lammer, J.-M. Grießmeier, M. Leitner, F. Selsis, C. Eiroa, A. Hanslmeier, H. K. Biernat, C. J. Farrugia, H. O. Rucker: CME activity of low mass $M$ stars as an important factor for the habitability of terrestrial exoplanets. Part I: CME impact on expected magnetospheres of Earth-like exoplanets in close-in habitable zones, Astrobiology, eingereicht (2006).

3. J.-M. Grießmeier, S. Preusse, M. Khodachenko, U. Motschmann, G. Mann, H. O. Rucker: Exoplanetary radio emission under different stellar wind conditions, Planet. Space Sci., eingereicht (2006).

4. M. L. Khodachenko, H. Lammer, H. I. M. Lichtenegger, D. Langmayr, N. V. Erkaev, J.-M. Grießmeier, M. Leitner, T. Penz, H. K. Biernat, U. Motschmann, H. O. Rucker: Mass loss of "Hot Jupiters" - Implications for CoRoT discoveries. Part I: The importance of magnetospheric protection of a planet against ion loss caused by Coronal Mass Ejections, Planet. Space Sci., eingereicht (2006).

5. D. Breuer, J.-M. Grießmeier, U. Motschmann, M. Khodachenko, C. Kolb, J. Parnell, T. Spohn, A. Stadelmann: Constraints on planetary habitability, in Report of the Terrestrial Exoplanet Science Advisory Team, (Hrsg.) M. Fridlund und Helmut Lammer, ESA-SP, im Druck (2006).

6. J.-M. Grießmeier, U. Motschmann, M. Khodachenko, H. O. Rucker: The influence of stellar coronal mass ejections on exoplanetary radio emission, in Planetary Radio Emissions VI, (Hrsg.) W. S. Kurth, G. Mann und H. O. Rucker, Austrian Academy of Sciences Press, Vienna, 571 (2006). 
7. J.-M. Grießmeier, U. Motschmann, K.-H. Glassmeier, G. Mann, H. O. Rucker: The potential of exoplanetary radio emissions as an observation method, in Tenth Anniversary of 51 Peg-b : Status of and Prospects for hot Jupiter studies, (Hrsg.) L. Arnold, F. Bouchy und C. Moutou, Platypus Press, 259 (2006).

8. J.-M. Grießmeier, A. Stadelmann, H. Lammer, N. Belisheva, U. Motschmann: The impact of galactic cosmic rays on extrasolar Earth-like planets in close-in habitable zones, in Proc. 39th ESLAB Symposium, (Hrsg.) F. Favata und A. Gimenez, ESA SP 588, 305 (2006).

9. M. L. Khodachenko, J.-M. Grießmeier, I. Ribas, H. Lammer, F. Selsis, M. Leitner, T. Penz, A. Hanslmeier, H. K. Biernat, H. O. Rucker: Habitability of the Earthlike exoplanets under the action of host stars intensive CME activity, in Proc. 39th ESLAB Symposium, (Hrsg.) F. Favata und A. Gimenez, ESA SP 588, 279 (2006).

10. H. Lammer, E. Chassefière, Yu. N. Kulikov, F. Leblanc, H. I. M. Lichtenegger, J.-M. Grießmeier, M. Khodachenko, D. Stam, C. Sotin, I. Ribas, F. Selsis, F. Allard, I. Mingalev, O. Mingalev, H. Rauer,J. L. Grenfell, D. Langmayr, G. Jaritz, S. Endler, G. Wuchterl, S. Barabash, H. Gunell, R. Lundin, H. K. Biernat, H. O. Rucker, F. Westall, A. Brack, S. J. Bauer, A. Hanslmeier, P. Odert, M. Leitzinger, P. Wurz, E. Lohinger, R. Dvorak, W. W. Weiss, W. von Bloh, S. Franck, T. Penz, A. Stadelmann, U. Motschmann, N. K. Belisheva, A. Bérces, A. Léger, C. S. Cockell, J. Parnell, I. L. Arshukova, N. V. Erkaev, A. A. Konovalenko, E. Kallio, G. Horneck, T. Guillot, A. Morbidelli, E. Bois, P. Barge, M. Deleuil, C. Moutou, F. Forget, B. Érdi, A. Hatzes, E. Szuszkiewicz, M. Fridlund: Towards real comparative planetology: Synergies between solar system science and the DARWIN mission, in Proc. 39th ESLAB Symposium, (Hrsg.) F. Favata und A. Gimenez, ESA SP 588, 233 (2006).

11. N. V. Erkaev, T. Penz, H. Lammer, H. I. M. Lichtenegger, H. K. Biernat, P. Wurz, J.-M. Grießmeier, W. W. Weiss: Plasma and magnetic field parameters in the vicinity of short-periodic giant exoplanets, Astrophys. J. Suppl. Ser. 157, 396 (2005).

12. J.-M. Grießmeier, U. Motschmann, G. Mann, H. O. Rucker: The influence of stellar wind conditions on the detectability of planetary radio emissions, Astron. Astrophys. 437, 717 (2005).

13. J.-M. Grießmeier, A. Stadelmann, U. Motschmann, N. K. Belisheva, H. Lammer, H. K. Biernat: Cosmic ray impact on extrasolar Earth-like planets in close-in habitable zones, Astrobiology 5, 587 (2005).

14. G. Jaritz, S. Endler, D. Langmayr, H. Lammer, J.-M. Grießmeier, N. V. Erkaev, H. K. Biernat: Roche lobe effects on expanded upper atmospheres of short-periodic giant planets, Astron. Astrophys. 439, 771 (2005).

15. A. S. Lipatov, U. Motschmann, T. Bagdonat, J.-M. Grießmeier: The interaction of the stellar wind with an extrasolar planet - 3D hybrid and drift-kinetic simulation, Planet. Space. Sci. 53, 423-432 (2005). 
16. H. Lammer, I. Ribas, J.-M. Grießmeier, T. Penz, A. Hanslmeier, H. K. Biernat: A brief history of the solar radiation and particle flux evolution, Hvar Obs. Bull. 28, 139 (2004).

17. J.-M. Grießmeier, A. Stadelmann, T. Penz, H. Lammer, F. Selsis, I. Ribas, E. F. Guinan, U. Motschmann, H. K. Biernat, W. W. Weiss: The effect of tidal locking on the magnetospheric and atmospheric evolution of "Hot Jupiters", Astron. Astrophys. 425, 753 - 762 (2004).

Tagungsbeiträge:

1. J.-M. Grießmeier, A. Stadelmann, H. Lammer, N. Belisheva, U. Motschmann: The impact of galactic cosmic rays through the magnetospheres of different extrasolar planets, Vortrag, 5th European Workshop on Astrobiology, Budapest (2005).

2. J.-M. Grießmeier, U. Motschmann, K.-H. Glassmeier, G. Mann , H. Rucker: The potential of exoplanetary radio emission as an observation method, Vortrag, Tenth Anniversary of 51 Peg-b: status of and prospects for hot Jupiter studies, An international colloquium, Observatoire de Haute Provence (2005).

3. J.-M. Grießmeier, U. Motschmann, K.-H. Glassmeier, G. Mann, H. Rucker: The influence of stellar system age on exoplanetary radio emission, Vortrag, EGU General Assembly, Wien (2005).

4. J.-M. Grießmeier, S. Preusse, U. Motschmann, M. Khodachenko, H. O. Rucker: Exoplanetary radio emissions under different stellar wind conditions, Vortrag, Workshop Planetary Radio Emission VI, Graz (2005).

5. J.-M. Grießmeier, A. Stadelmann, H. Lammer, N. Belisheva, U. Motschmann: The impact of galactic cosmic rays on extrasolar Earth-like planets in close-in habitable zones, Poster, ESLAB Symposium "Trends in Space Science and Cosmic Vision 2020”, ESTEC, Noordwijk (2005).

6. J.-M. Grießmeier, A. Stadelmann, S. Preusse, H. Lammer, T. Penz, F. Selsis, U. Motschmann: Extrasolar magnetospheres under different stellar wind conditions, Vortrag, 3. Workshop "Planetenbildung: Das Sonnensystem und extrasolare Planeten", Münster (2004).

7. J.-M. Grießmeier, A. Stadelmann, H. Lammer, F. Selsis, U. Motschmann: Tidal locking and its influence on planetary habitability, Poster, Bioastronomy 2004, Reykjavik (2004).

8. T. Penz, H. Lammer, J.-M. Grießmeier, U. V. Amerstorfer, F. Selsis, I. Ribas, H. K. Biernat: Atmospheric evolution of non-magnetized terrestrial exoplanets, Poster, Bioastronomy 2004, Reykjavik (2004).

9. J.-M. Grießmeier, A. Stadelmann, H. Lammer, U. Motschmann: Tidal locking and its influence on exomagnetospheres, Vortrag, Planet Workshop "Close-in exoplanets: the star-planet connection" of the Corot week 6, Orsay (2004). 
10. D. F. Vogl, T. Penz, J.-M. Grießmeier, H. Lammer, N. V. Erkaev. G. F. Jaritz, M. G. Therany, H. K. Biernat, A. Hanslmeier, W. W. Weiss: Are there bow shocks around short period extrasolar gas giants?, Poster, EGU General Assembly, Nizza (2004).

11. J.-M. Grießmeier, U. Motschmann, K.-H. Glassmeier, G. Mann, H. Rucker: Discrimination of exoplanetary and stellar radio flux for different stellar wind conditions, Vortrag, EGU General Assembly, Nizza (2004).

12. J.-M. Grießmeier, U. Motschmann, A. Stadelmann, T. Penz, H. Lammer: Der Einfluß gebundener Rotation auf Magnetosphären und Atmosphären von Exoplaneten, Vortrag, Tagung der Arbeitsgemeinschaft Extraterrestrische Forschung, Kiel (2004).

13. D. F. Vogl, T. Penz, J-M. Grießmeier, H. Lammer, N. V. Erkaev, G. F. Jaritz, M. G. Therany, H. K. Biernat, A. Hanslmeier, W. W. Weiss: Plasma and magnetic field parameters at bow shocks of short period extrasolar gas giants, Poster, 27th Annual Seminar "Physics of Auroral Phenomena" in Apatity, Russia (2004).

14. J.-M. Grießmeier, U. Motschmann, G. Mann: Discrimination of exoplanetary and stellar radio flux, Poster, Corot week 5, Berlin (2003).

15. T. Penz, A. Stadelmann, H. Lammer, J.-M. Grießmeier, F. Selsis, H. K. Biernat, A. Hanslmeier: The influence of the interior structure of Uranus-type extrasolar planets on the stellar wind interaction, Poster, Corot week 5, Berlin (2003).

16. D. F. Vogl, T. Penz, H. Lammer, J.-M. Grießmeier, H. K. Biernat, G. J. Jaritz, M. G. Therany, N. V. Erkaev, A. Hanslmeier, W. W. Weiss: Plasma and field parameters across the bow shock of Jupiter-type exoplanets in the vicinity of their host star, Poster, Corot week 5, Berlin (2003).

17. T. Penz, J.-M. Griessmeier, A. Stadelmann, H. Lammer, H. I. M. Lichtenegger, F. Selsis, I. Ribas, H. K. Biernat, W. W. Weiss: Magnetosphere-stellar wind interaction of "Hot Jupiters", Poster, Corot week 4, Marseille (2003).

18. J.-M. Grießmeier, U. Motschmann, K.-H. Glassmeier: Exomagnetospheres and their interaction with the stellar wind, Vortrag, EGS-AGU-EUG Joint Assembly, Nizza (2003).

19. J.-M. Grießmeier, U. Motschmann, K.-H. Glaßmeier: Exomagnetosphären und ihre Wechselwirkung mit dem Sternenwind, Vortrag, Tagung der Arbeitsgemeinschaft Extraterrestrische Forschung, Jena (2003). 


\section{Contents}

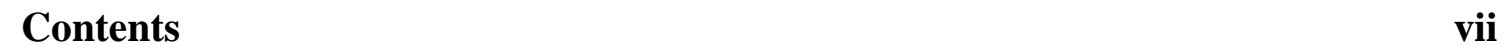

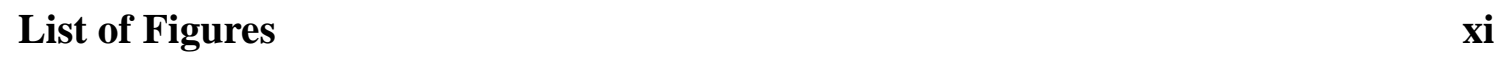

List of Tables $\quad$ xiii

\begin{tabular}{|l|l|l|l}
\hline List of symbols and constants & xv
\end{tabular}

\begin{tabular}{ll}
\hline Abstract & xix
\end{tabular}

\begin{tabular}{lll}
\hline 1 & Introduction & 1
\end{tabular}

\begin{tabular}{|lll}
2 & Extrasolar planets: An overview & 5
\end{tabular}

2.1 Historical development . . . . . . . . . . . . . . . . . 5

2.2 Definitions . . . . . . . . . . . . . . . . . 6

2.2.1 Extrasolar planets: working definition of the IAU . . . . . . . . 6

2.2.2 $\quad$ Extrasolar planets: alternative definition of G. Basri . . . . . . . . 7

$2.2 .3 \quad$ Extrasolar planets: definition used in this work $\ldots . . . . .77$

2.2 .4 Hot Jupiters . . . . . . . . . . . . . . . . . . . . . . . . . . . . . . . .

2.2.5 Terrestrial Exoplanets . . . . . . . . . . . . . 8

2.3 The habitable zone . . . . . . . . . . . . . . . . 8

2.4 Overview of current observation methods . . . . . . . . . . . . . 11

2.5 Chromospheric heating . . . . . . . . . . . . . . . . . . . . 14

2.6 Planetary parameters $\ldots \ldots \ldots \ldots \ldots$. . . . . . . . . . . . . . . . . . . . . . . . . .

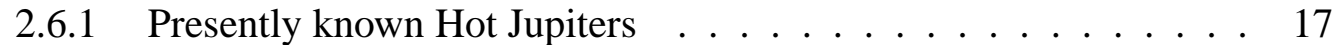

$2.6 .2 \quad$ Parameters for Hot Jupiters . . . . . . . . . . . . . . . . . . 18

2.6 .3 Presently known terrestrial exoplanets . . . . . . . . . . . . . 20

2.6 .4 Parameters for terrestrial exoplanets . . . . . . . . . . . . 20

3 Tidal interaction 23

3.1 Tidal locking . . . . . . . . . . . . . . . . . . 25

3.1.1 Tidal locking timescale . . . . . . . . . . . . . . . 26

3.1.2 Imperfect tidal locking . . . . . . . . . . . . . . . . . 28

3.1.3 Parameters for gas giants . . . . . . . . . . . . . . . . . . . . . . . . . . 29

3.1.3.1 Structure parameter $\alpha \ldots \ldots$. . . . . . . . . . . . . . . . . . 29

3.1.3.2 Tidal dissipation factor $Q_{\mathrm{p}}^{\prime} \ldots \ldots \ldots \ldots$

3.1.3.3 Initial rotation rate $\omega_{\mathrm{i}} \ldots \ldots \ldots \ldots$ 
3.1.3.4 Final rotation rate $\omega_{\mathrm{f}} \ldots \ldots \ldots \ldots \ldots$

3.1.3.5 Overview of the parameters . . . . . . . . . . . 31

3.1 .4 Results for gas giants . . . . . . . . . . . . . . . . 31

3.1 .5 Parameters for terrestrial planets . . . . . . . . . . . . . . 34

3.1.5.1 Structure parameter $\alpha \ldots \ldots \ldots 34$

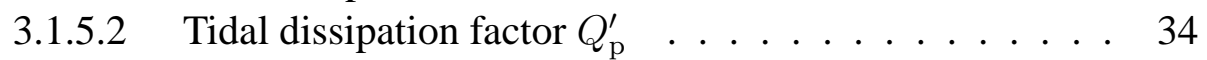

3.1.5.3 Initial rotation rate $\omega_{\mathrm{i}} \ldots \ldots \ldots \ldots \ldots$

$3.1 .5 .4 \quad$ Final rotation rate $\omega_{\mathrm{f}} \ldots \ldots \ldots \ldots \ldots . \ldots . \ldots 35$

3.1 .5 .5 Overview of the parameters . . . . . . . . . . . . 36

3.1 .6 Results for terrestrial planets . . . . . . . . . . . . . 36

3.2 Orbital circularisation . . . . . . . . . . . . . . . . . . 40

3.3 Obliquity damping . . . . . . . . . . . . . . . . . . . . 42

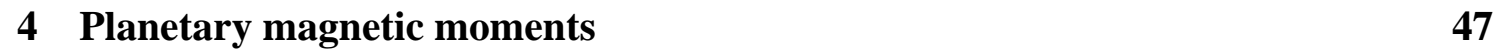

4.1 Magnetic moment scaling laws . . . . . . . . . . . . . . . . . . . . . 48

4.1 .1 Blackett's law . . . . . . . . . . . . . . . . . . . . . . . . 50

4.1 .2 Busse's geostrophic scaling law . . . . . . . . . . . . . 50

$4.1 .3 \quad$ Scale analysis by Jacobs $\ldots \ldots \ldots$. . . . . . . . . . . . . 51

4.1 .4 Stevenson's scaling based on heat flow . . . . . . . . . . . . 51

$4.1 .5 \quad$ Scaling law of Curtis \& Ness . . . . . . . . . . . . . . . . . . . 52

4.1 .6 Mizutani's scaling law . . . . . . . . . . . . . . . . 53

4.1 .7 Sano's scaling law $\ldots \ldots \ldots \ldots$. . . . . . . . . . . . . 54

4.1 .8 Scaling law based on the Elsasser Number. . . . . . . . . . . . . . 54

4.1 .9 Overview over the scaling laws . . . . . . . . . . . . . . . . 55

4.2 Limits of the scaling law concept . . . . . . . . . . . . . . . 56

4.3 Input parameters for gas giants $\ldots \ldots \ldots \ldots$. . . . . . . . . . 58

4.3 .1 The hydrostatic model . . . . . . . . . . . . . . . . 59

4.3 .2 Size of the dynamo region $r_{\mathrm{c}} \ldots \ldots \ldots 62$

4.3 .3 Density of the dynamo region $\rho_{\mathrm{c}} \ldots \ldots \ldots$. . . . . . . . . . . . . . . 67

4.3.4 Planetary rotation rate $\omega \ldots \ldots$. . . . . . . . . . . . . 69

4.3 .5 Conductivity within the dynamo region $\sigma \ldots \ldots$. . . . . . . 70

4.3 .6 Known planetary parameters . . . . . . . . . . . . . . 70

4.4 Scaling results for gas giants . . . . . . . . . . . . . . . . . 71

4.5 Input parameters for terrestrial planets $\ldots \ldots \ldots \ldots 76$

4.5 .1 Planetary models . . . . . . . . . . . . . . . 76

4.5.2 Size of the dynamo region $r_{\mathrm{c}} \ldots \ldots \ldots \ldots \ldots$

$4.5 .3 \quad$ Density of the dynamo region $\rho_{\mathrm{c}} \ldots \ldots \ldots \ldots 77$

$4.5 .4 \quad$ Planetary rotation rate $\omega \ldots \ldots . \ldots 77$

4.5.5 Conductivity within the dynamo region $\sigma \ldots \ldots 78$

4.5 .6 Planetary structure . . . . . . . . . . . . . . . . . 78

4.6 Scaling results for terrestrial planets $\ldots \ldots \ldots \ldots$

5 Formation of magnetospheres by stellar winds 85

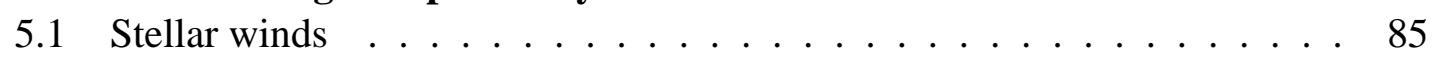

5.1 .1 Radial dependence . . . . . . . . . . . . . . . 86

5.1.1.1 Stellar wind model of Parker . . . . . . . . . . 86 
5.1.1.2 Stellar wind model of Weber and Davis . . . . . . . . 87

5.1 .2 Long term time dependence . . . . . . . . . . . . . . . . . . 89

5.1 .3 Influence of the orbital velocity . . . . . . . . . . . . . . . . . . 91

5.1 .4 Resulting stellar wind parameters . . . . . . . . . . . . . . . 93

5.2 Stellar coronal mass ejections . . . . . . . . . . . . . . . . 95

5.2 .1 Density, velocity and temperature . . . . . . . . . . . . . . . . 95

5.2 .2 Occurrence rate . . . . . . . . . . . . . . . . . . . . 97

5.2 .3 Comparison to stellar wind parameters . . . . . . . . . . . . . 98

5.3 Planetary magnetospheres $\ldots \ldots \ldots$. . . . . . . . . . . . . . 98

$5.3 .1 \quad$ Magnetospheric model . . . . . . . . . . . . . . . . . . . . . . 100

5.3 .2 Pressure equilibrium . . . . . . . . . . . . . . . . . 102

5.3.2.1 $\quad$ Stellar wind kinetic pressure . . . . . . . . . . . . 104

5.3.2.2 Stellar wind magnetic pressure . . . . . . . . . . . . 104

5.3 .2 .3 Stellar wind thermal pressure . . . . . . . . . . . . 105

5.3 .2 .4 Planetary magnetic pressure . . . . . . . . . . . . . . 106

5.3.2.5 Planetary plasma thermal pressure . . . . . . . . . 107

5.3 .2 .6 Pressure balance . . . . . . . . . . . . . . 107

5.3 .3 Size of the magnetosphere of gas giants . . . . . . . . . . . 108

5.3 .4 Size of the magnetosphere of terrestrial planets . . . . . . . . 111

\begin{tabular}{|lll}
\hline 6 & Nonthermal radio emission from the magnetospheres of Hot Jupiters & 117
\end{tabular}

6.1 Planetary radio emission . . . . . . . . . . . . . . . . 118

6.1 .1 Planetary radio emission in the solar system . . . . . . . . . . 118

6.1 .2 Model of exoplanetary radio emission . . . . . . . . . . . . . . 120

6.1 .3 Influence of the stellar system age . . . . . . . . . . . . . . . . 126

6.1 .4 Influence of stellar CMEs . . . . . . . . . . . . . . . . . . . . 129

6.2 Solar and stellar radio emission . . . . . . . . . . . . . . . . . . 132

6.2 .1 Solar radio emission . . . . . . . . . . . . . . . . . . . . . . . . . . . 132

6.2 .2 Stellar radio emission . . . . . . . . . . . . . . . . . . . . 134

6.2 .3 Comparison of solar, stellar and exoplanetary radio fluxes . . . . 135

6.3 Observation of exoplanetary radio emission . . . . . . . . . . . . 138

6.3 .1 Observational attempts . . . . . . . . . . . . . . . . . 138

6.3 .2 Estimated radio flux . . . . . . . . . . . . . . . . . 140

\begin{tabular}{|lll}
\hline & Protection of terrestrial exoplanets against galactic cosmic rays & $\mathbf{1 4 3}$
\end{tabular}

$7.1 \quad$ Galactic cosmic rays . . . . . . . . . . . . . . . . . . . . . . 144

7.2 Cosmic ray calculation . . . . . . . . . . . . . . . . . . 144

7.2.1 Calculation of particle trajectories . . . . . . . . . . . . . 144

7.2 .2 Cosmic ray impact area . . . . . . . . . . . . . . . . . . . 146

$7.2 .3 \quad$ Cosmic ray energy spectrum . . . . . . . . . . . . . . . 146

7.3 Cosmic rays in exomagnetospheres . . . . . . . . . . . . . . . . 147

7.3.1 Impact of cosmic rays on Earth-like exoplanets . . . . . . . . . 147

7.3 .2 Influence of tidal locking . . . . . . . . . . . . . . . . . . . . . . . . 148

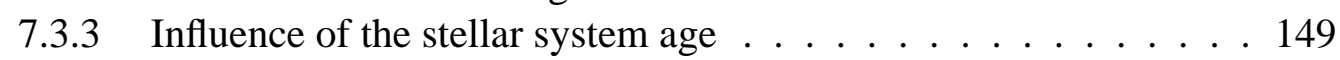

7.3 .4 Influence of the type of planet . . . . . . . . . . . . . . . . . . . 149

7.4 Implications for habitability . . . . . . . . . . . . . . 150 
\begin{tabular}{lll}
\hline & Conclusions & 157
\end{tabular}

\begin{tabular}{lr}
\hline Bibliography & 159
\end{tabular} 


\section{List of Figures}

$1.1 \quad$ Interaction of exomagnetospheres with the stellar wind . . . . . . . . 2

2.1 Habitable zone . . . . . . . . . . . . . . . . . . . 10

3.1 Tidal interaction (schematic) . . . . . . . . . . . . . . 26

3.2 Tidal locking regimes (Hot Jupiters) $\ldots \ldots \ldots$. . . . . . . . . . . 32

3.3 Tidal locking regimes (HD 209458b) . . . . . . . . . . . . . . . . . . . . . . . . 33

3.4 Tidal locking regimes $(\tau$ Bootes $b$, heavy model $) \quad \ldots \ldots . \ldots . \ldots 33$

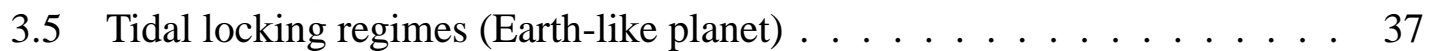

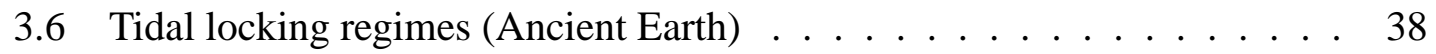

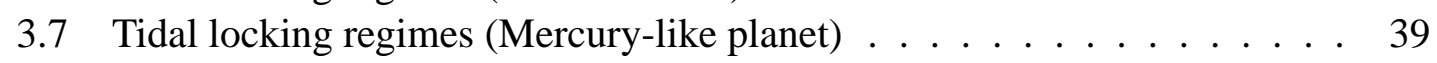

3.8 Tidal locking regimes (Large Earth) $\ldots \ldots \ldots$. . . . . . . . . . . . . 39

3.9 Tidal locking regimes (Ocean Planet) $\ldots \ldots \ldots$. . . . . . . . . . . 40

3.10 Orbital circularisation timescale (Jupiter and Earth) . . . . . . . . . . . . 43

3.11 Orbital circularisation timescale (Mercury) . . . . . . . . . . . . . . . . 44

$4.1 \quad$ Density of the planetary interior $\ldots \ldots \ldots$. . . . . . . . . 61

4.2 Size of the dynamo region: limit of the scaling approximation . . . . . . 64

4.3 Size of the dynamo region: limit of the hydrostatic approximation . . . . 65

$4.4 \quad$ Average density of the planetary core . . . . . . . . . . . . . 68

$4.5 \quad$ Magnetic moment (Jupiter-like) $\ldots \ldots \ldots$. . . . . . . . . . . 74

4.6 Magnetic moment (HD 209458b) . . . . . . . . . . . . . . . . . . . . . 75

4.7 Magnetic moment $(\tau$ Bootes b, heavy model $) \ldots \ldots$. . . . . . . . . 75

4.8 Magnetic moment (Earth-like planet) . . . . . . . . . . . . . . . . 81

4.9 Magnetic moment (Mercury-like planet) . . . . . . . . . . . . . . . . 82

4.10 Magnetic moment (Large Earth) . . . . . . . . . . . . . . . . . . . . . . . . 82

4.11 Magnetic moment (Ocean Planet) . . . . . . . . . . . . . . . . 83

$5.1 \quad$ Solar wind velocity and density $\ldots \ldots \ldots$. . . . . . . . . . . 88

5.2 Time evolution of stellar wind parameters . . . . . . . . . . . . . 92

$5.3 \quad$ Stellar wind velocity and density . . . . . . . . . . . . . . . . . . 99

5.4 Geometry of the magnetosphere (schematic) . . . . . . . . . . . . . . . . 101

5.5 Magnetospheric magnetic field . . . . . . . . . . . . . . . . . . . 103

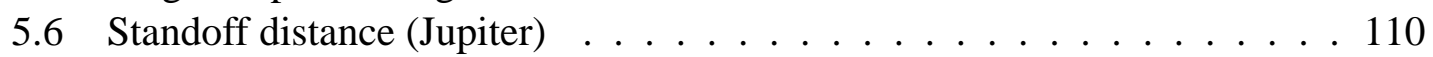

5.7 Standoff distance (HD 209458b) $\ldots \ldots$. . . . . . . . . . . . . . . . . 110

5.8 Standoff distance $(\tau$ Bootes b, heavy model $)$. . . . . . . . . . . . . . . . 111

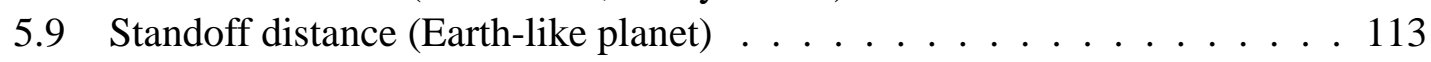




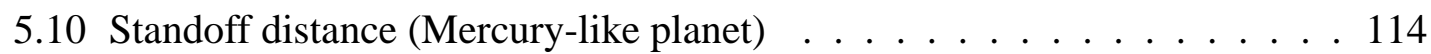

5.11 Standoff distance (Large Earth) . . . . . . . . . . . . . . . . . . . . . . 114

5.12 Standoff distance $($ Ocean Planet $)$. . . . . . . . . . . . . . . . . 115

6.1 Jupiter's radio emission . . . . . . . . . . . . . . . . . . . . . . . . . . . 119

6.2 Exoplanetary radio emission (stellar system age) . . . . . . . . . . . . 128

6.3 Exoplanetary radio emission (stellar coronal mass ejections) . . . . . . . 131

6.4 Solar radio emission $\ldots \ldots \ldots \ldots . \ldots \ldots$

6.5 Stellar and planetary radio emission (comparison) . . . . . . . . . . . . . 137

6.6 Exoplanetary radio emission (observational attempts) . . . . . . . . . . . 141

7.1 Starting configuration of cosmic ray particles (schematic) . . . . . . . . 145

7.2 Particle impact region . . . . . . . . . . . . . . . . . . . . 146

7.3 Impact area: exoplanet around a K/M star . . . . . . . . . . . . . . . . . 152

7.4 Energy spectrum: exoplanet around a K/M star . . . . . . . . . . . . . 152

7.5 Impact area: influence of tidal locking . . . . . . . . . . . . . . 153

7.6 Energy spectrum: influence of tidal locking . . . . . . . . . . . . . . 153

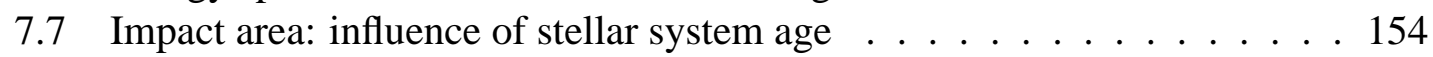

7.8 Energy spectrum: influence of stellar system age . . . . . . . . . . . . 154

7.9 Impact area: influence of planetary type . . . . . . . . . . . . . 155

7.10 Energy spectrum: influence of planetary type . . . . . . . . . . 155 


\section{List of Tables}

$1 \quad$ Frequently used mathematical symbols and physical constants. . . . . . xv

2.1 Planetary observation methods . . . . . . . . . . . . . . . . 15

2.2 Planetary and stellar parameters (gas giants) . . . . . . . . . . . . . . . . . 21

2.3 Planetary and stellar parameters (terrestrial planets) . . . . . . . . . . 22

3.1 Tidal locking: input parameters (Hot Jupiters) . . . . . . . . . . . . . . . 31

3.2 Tidal locking: input parameters (terrestrial planets) . . . . . . . . . . . 36

4.1 Size of the dynamo region: comparison . . . . . . . . . . . . . 66

4.2 Density in the dynamo region: comparison . . . . . . . . . . . . . . . . 69

4.3 Magnetic moment scaling: input parameters (gas giants) . . . . . . . . 71

4.4 Magnetic moment scaling: results (gas giants) . . . . . . . . . . . . . 72

4.5 Magnetic moment scaling: planetary rotation rates (terrestrial planets) . . 78

$4.6 \quad$ Magnetic moment scaling: input parameters (terrestrial planets) . . . . . 79

$4.7 \quad$ Magnetic moment scaling: results (terrestrial planets) $\ldots . . . . . . .880$

$5.1 \quad$ Stellar wind parameters $(\mathrm{G} \mathrm{star}) \quad \ldots \ldots \ldots$. . . . . . . . . . . . . . . . . . . . . . . . . . . 94

5.2 Stellar wind parameters (K star) $\ldots \ldots \ldots$. . . . . . . . . . 95

5.3 Parameters typical for strong CME . . . . . . . . . . . . . . . . . 100

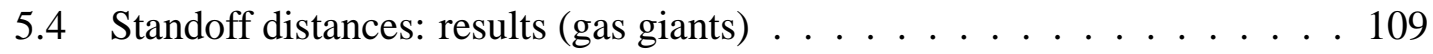

5.5 Standoff distances: results (terrestrial planets) . . . . . . . . . . . . . . . 112

$6.1 \quad$ Jupiter's total radio power . . . . . . . . . . . . . . . . . . . . . . . 122

6.2 Planetary radio flux (comparison of planets) . . . . . . . . . . . . . . . . . 124

6.3 Planetary radio flux (time dependence) . . . . . . . . . . . . . . . . . 127

6.4 Planetary radio flux (coronal mass ejections) . . . . . . . . . . . . . . . 130

6.5 Past observational attempts $\ldots \ldots \ldots$. . . . . . . . . . . . . . . . 139

7.1 Magnetospheric parameters for cosmic ray calculation . . . . . . . . . 148 


\section{List of symbols and constants}

Table 1: Frequently used mathematical symbols and physical constants.

\begin{tabular}{|c|c|c|}
\hline Symbol & Significance & Unit \\
\hline $\mathrm{AU}$ & astronomical unit $\left(1.49597870 \cdot 10^{11} \mathrm{~m}\right)$ & $\mathrm{m}$ \\
\hline$B$ & magnetic field & $\mathrm{nT}, \mathrm{T}$ \\
\hline$B_{\mathrm{cf}}$ & magnetic field due to Chapman-Ferraro currents & $\mathrm{nT}, \mathrm{T}$ \\
\hline$B_{\mathrm{p}}$ & magnetic field due to planetary magnetic dipole moment & $\mathrm{nT}, \mathrm{T}$ \\
\hline$B_{\mathrm{p}}^{\max }$ & planetary magnetic field at polar cloud tops & $\mathrm{nT}, \mathrm{T}$ \\
\hline$d$ & semi-major axis of planetary orbit & $\mathrm{AU}$ \\
\hline$e$ & orbital eccentricity & 1 \\
\hline$e$ & elementary charge $\left(1.60217733 \cdot 10^{-19} \mathrm{C}\right)$ & $\mathrm{C}$ \\
\hline$E$ & heat flux through a planet & $\mathrm{J} \mathrm{m}^{-2} \mathrm{~s}^{-1}$ \\
\hline$f$ & frequency of radio emission & $\mathrm{Hz}, \mathrm{MHz}$ \\
\hline$f_{0}$ & magnetospheric form factor & 1 \\
\hline$f_{\mathrm{c}}^{\max }$ & maximum frequency of planetary radio emission & $\mathrm{Hz}, \mathrm{MHz}$ \\
\hline$f_{\mathrm{CME}}$ & CME generation frequency & 1/day \\
\hline$f_{\mathrm{CME}}^{\mathrm{c}}$ & critical CME generation frequency for continuous influence & 1/day \\
\hline$f_{\text {impact }}$ & CME impact frequency & 1/day \\
\hline$g$ & gravitational acceleration & $\mathrm{m} / \mathrm{s}^{2}$ \\
\hline$G$ & constant of gravitation $\left(6.6725985 \cdot 10^{-11} \mathrm{Nm}^{2} \mathrm{~kg}^{-2}\right)$ & $\mathrm{Nm}^{2} \mathrm{~kg}^{-2}$ \\
\hline Gyr & billion years $\left(10^{9} \mathrm{yr}\right)$ & $\mathrm{yr}$ \\
\hline$i$ & inclination of planetary orbit & $\circ$ \\
\hline$I_{a}$ & cosmic ray differential spectral intensity & $\left(\mathrm{m}^{2} \mathrm{srs} \mathrm{MeV}\right)^{-1}$ \\
\hline$k_{2, \star}$ & stellar Love number & 1 \\
\hline$k_{2, \mathrm{p}}$ & planetary Love number & 1 \\
\hline$k_{\mathrm{B}}$ & Boltzmann's constant $\left(1.380658 \cdot 10^{-23} \mathrm{~J} / \mathrm{K}\right)$ & $\mathrm{J} / \mathrm{K}$ \\
\hline$L_{\odot}$ & solar luminosity & $\mathrm{W}$ \\
\hline$L_{\star}$ & stellar luminosity & $\mathrm{W}$ \\
\hline$m$ & proton rest mass $\left(1.660540210 \cdot 10^{-27} \mathrm{~kg}\right)$ & $\mathrm{kg}$ \\
\hline$m_{\mathrm{e}}$ & electron rest mass $\left(9.1093897 \cdot 10^{-31} \mathrm{~kg}\right)$ & $\mathrm{kg}$ \\
\hline
\end{tabular}




\begin{tabular}{|c|c|c|}
\hline \multicolumn{3}{|c|}{ continued from previous page } \\
\hline Symbol & Significance & Unit \\
\hline $\mathcal{M}$ & planetary magnetic moment & $\mathrm{Am}^{2}$ \\
\hline $\mathcal{M}_{\mathrm{dr}}$ & planetary magnetic moment (planet with doubled rotation) & $\mathrm{Am}^{2}$ \\
\hline $\mathcal{M}_{\mathrm{E}}$ & magnetic moment of the Earth $\left(7.91 \cdot 10^{22} \mathrm{Am}^{2}\right)$ & $\mathrm{Am}^{2}$ \\
\hline $\mathcal{M}_{\mathrm{J}}$ & magnetic moment of Jupiter $\left(1.56 \cdot 10^{27} \mathrm{Am}^{2}\right)$ & $\mathrm{Am}^{2}$ \\
\hline $\mathcal{M}_{\text {rr }}$ & planetary magnetic moment (rapidly rotating planet) & $\mathrm{Am}^{2}$ \\
\hline$M_{\odot}$ & solar mass $\left(1.98911 \cdot 10^{30} \mathrm{~kg}\right)$ & $\mathrm{kg}$ \\
\hline$M_{\star}$ & stellar mass & $\mathrm{kg}$ \\
\hline$M_{\mathrm{E}}$ & mass of the Earth $\left(6.0 \cdot 10^{24} \mathrm{~kg}\right)$ & $\mathrm{kg}$ \\
\hline$M_{\mathrm{J}}$ & mass of Jupiter $\left(1.9 \cdot 10^{27} \mathrm{~kg}\right)$ & $\mathrm{kg}$ \\
\hline$M_{\mathrm{p}}$ & planetary mass & $\mathrm{kg}$ \\
\hline$M_{r}$ & mass contained within sphere of radius $r$ & $\mathrm{~kg}$ \\
\hline Myr & million years $\left(10^{6} \mathrm{yr}\right)$ & $\mathrm{yr}$ \\
\hline$n$ & stellar wind number density & $\mathrm{m}^{-3}$ \\
\hline$n_{\mathrm{J}}$ & stellar wind number density at Jupiter's orbit & $\mathrm{m}^{-3}$ \\
\hline$n_{\mathrm{CME}}^{\mathrm{s}}$ & number density of strong CME & $\mathrm{m}^{-3}$ \\
\hline$n_{\mathrm{CME}}^{\mathrm{w}}$ & number density of weak CME & $\mathrm{m}^{-3}$ \\
\hline$p$ & pressure & $\mathrm{Nm}^{-2}$ \\
\hline$p_{\text {imf }}$ & stellar wind magnetic pressure & $\mathrm{Nm}^{-2}$ \\
\hline$p_{\mathrm{m}}$ & planetary magnetic pressure & $\mathrm{Nm}^{-2}$ \\
\hline$p_{\mathrm{sw}}$ & stellar wind kinetic pressure & $\mathrm{Nm}^{-2}$ \\
\hline$p_{\text {th }}$ & stellar wind thermal pressure & $\mathrm{Nm}^{-2}$ \\
\hline$p_{\mathrm{th}, \mathrm{m}}$ & planetary thermal pressure & $\mathrm{Nm}^{-2}$ \\
\hline$P_{\text {input }}$ & power input into magnetosphere & W \\
\hline$P_{\text {rad }}$ & total power of planetary radio emission & $\mathrm{W}$ \\
\hline$P_{\text {rad,CME }}$ & total power of planetary radio emission due to $\mathrm{CME}$ & $\mathrm{W}$ \\
\hline$P_{\mathrm{rad}, \mathrm{CME}}^{\mathrm{s}}$ & total power of planetary radio emission due to strong CME & $\mathrm{W}$ \\
\hline$P_{\text {rad,J }}$ & total power of Jupiter's radio emission & $\mathrm{W}$ \\
\hline$P_{\text {rot }}$ & stellar rotation period & s, days \\
\hline $\mathrm{pc}$ & $\operatorname{parsec}\left(3.08568 \cdot 10^{16} \mathrm{~m}\right)$ & $\mathrm{m}$ \\
\hline$Q_{\star}^{\prime}$ & modified tidal dissipation factor of the star & 1 \\
\hline$Q_{\mathrm{p}}$ & tidal dissipation factor of the planet & 1 \\
\hline$Q_{\mathrm{p}}^{\prime}$ & modified tidal dissipation factor of the planet & 1 \\
\hline$r$ & radial position & $\mathrm{m}$ \\
\hline$r_{\mathrm{c}, \mathrm{E}}$ & size of the dynamo region for the Earth & $\mathrm{m}$ \\
\hline$r_{\mathrm{c}, \mathrm{J}}$ & size of the dynamo region for Jupiter & $\mathrm{m}$ \\
\hline$r_{\mathrm{c}}$ & size of the dynamo region & $\mathrm{m}$ \\
\hline$r_{\text {crit }}$ & critical radius of the Parker stellar wind model & $\mathrm{m}$ \\
\hline
\end{tabular}




\begin{tabular}{|c|c|c|}
\hline \multicolumn{3}{|c|}{ continued from previous page } \\
\hline Symbol & Significance & Unit \\
\hline$R_{\odot}$ & solar radius $\left(6.96 \cdot 10^{8} \mathrm{~m}\right)$ & $\mathrm{m}$ \\
\hline$R_{\star}$ & stellar radius & $\mathrm{m}$ \\
\hline$R_{\mathrm{E}}$ & radius of the Earth $\left(6371 \cdot 10^{3} \mathrm{~m}\right)$ & $\mathrm{m}$ \\
\hline$R_{\mathrm{J}}$ & radius of Jupiter $\left(71492 \cdot 10^{3} \mathrm{~m}\right)$ & $\mathrm{m}$ \\
\hline$R_{\mathrm{mag}}$ & magnetic Reynolds number & 1 \\
\hline$R_{\mathrm{p}}$ & planetary radius & $\mathrm{m}$ \\
\hline$R_{\mathrm{s}}$ & substellar standoff distance of planetary magnetosphere & $\mathrm{m}$ \\
\hline$R_{\mathrm{s}, \mathrm{J}}$ & substellar standoff distance of Jupiter's magnetosphere & $\mathrm{m}$ \\
\hline$R_{\mathrm{s}}^{0.7}$ & standoff distance (stellar wind of a 0.7 Gyr star) & $\mathrm{m}$ \\
\hline$R_{\mathrm{s}}^{1.0}$ & standoff distance (stellar wind of a $1.0 \mathrm{Gyr}$ star) & $\mathrm{m}$ \\
\hline$R_{\mathrm{s}}^{4.6}$ & standoff distance (stellar wind of a 4.6 Gyr star) & $\mathrm{m}$ \\
\hline$R_{\mathrm{s}}^{\mathrm{CME}}$ & standoff distance (stellar CME) & $\mathrm{m}$ \\
\hline$R_{\mathrm{M}}$ & radius of planetary magnetosphere & $\mathrm{m}$ \\
\hline$s$ & stellar distance & pc \\
\hline$S_{\odot}$ & solar energy flux (1360 W) & $\mathrm{W} / \mathrm{m}^{2}$ \\
\hline$S_{\star}$ & stellar energy flux & $\mathrm{W} / \mathrm{m}^{2}$ \\
\hline$S_{\text {eff,min }}$ & minimum $S_{\star} / S_{\odot}$ for habitable zone & 1 \\
\hline$S_{\text {eff,max }}$ & maximum $S_{\star} / S_{\odot}$ for habitable zone & 1 \\
\hline$t$ & time & yr, Myr, Gyr \\
\hline$t_{\star}$ & stellar age & yr, Myr, Gyr \\
\hline$T$ & temperature & $\mathrm{K}, \mathrm{MK}$ \\
\hline$T_{\text {corona }}$ & corona temperature & $\mathrm{K}, \mathrm{MK}$ \\
\hline$T_{\mathrm{CME}}$ & plasma temperature of a $\mathrm{CME}$ & $\mathrm{K}, \mathrm{MK}$ \\
\hline$v$ & stellar wind velocity & $\mathrm{m} / \mathrm{s}$ \\
\hline$v_{\text {conv }}$ & convection velocity & $\mathrm{m} / \mathrm{s}$ \\
\hline$v_{\mathrm{CME}}$ & CME velocity & $\mathrm{m} / \mathrm{s}$ \\
\hline$v_{\text {crit }}$ & critical velocity of the Parker stellar wind model & $\mathrm{m} / \mathrm{s}$ \\
\hline$v_{\text {eff }}$ & effective velocity of stellar wind relative to planet & $\mathrm{m} / \mathrm{s}$ \\
\hline$v_{\text {eff, CME }}$ & effective velocity of CME relative to planet & $\mathrm{m} / \mathrm{s}$ \\
\hline$v_{\text {eff, } \mathrm{J}}$ & effective velocity of stellar wind relative to Jupiter & $\mathrm{m} / \mathrm{s}$ \\
\hline$v_{\text {orbit }}$ & planetary orbital velocity & $\mathrm{m} / \mathrm{s}$ \\
\hline $\mathrm{yr}$ & year $\left(3.14 \cdot 10^{7} \mathrm{~s}\right)$ & year \\
\hline$\alpha$ & structure parameter (mass distribution within a planet) & 1 \\
\hline$\delta_{\mathrm{p}}$ & angular size of planet as seen from the star & $\circ$ \\
\hline$\Delta_{\mathrm{CME}}$ & angular size of CME & $\circ$ \\
\hline$\Delta f$ & frequency bandwidth of planetary radio emission & $\mathrm{Hz}, \mathrm{MHz}$ \\
\hline$\Theta$ & range of stellar latitude where CMEs are produced & $\circ$ \\
\hline
\end{tabular}


List of symbols and constants

\begin{tabular}{|c|c|c|}
\hline \multicolumn{3}{|c|}{ continued from previous page } \\
\hline Symbol & Significance & Unit \\
\hline$\kappa$ & polytropic index & 1 \\
\hline$\lambda=r / R_{\mathrm{p}}$ & fractional radius & 1 \\
\hline$\mu_{0}$ & vacuum permeability $\left(4 \pi \cdot 10^{-7} \mathrm{Vs} / \mathrm{Am}\right)$ & $\mathrm{Vs} / \mathrm{Am}$ \\
\hline$\rho$ & mass density & $\mathrm{kg} / \mathrm{m}^{3}$ \\
\hline $\bar{\rho}$ & average mass density & $\mathrm{kg} / \mathrm{m}^{3}$ \\
\hline$\rho_{\mathrm{c}}$ & mass density in the dynamo region & $\mathrm{kg} / \mathrm{m}^{3}$ \\
\hline$\rho_{\mathrm{c}, \mathrm{E}}$ & mass density in the dynamo region for the Earth & $\mathrm{kg} / \mathrm{m}^{3}$ \\
\hline$\rho_{\mathrm{c}, \mathrm{J}}$ & mass density in the dynamo region for Jupiter & $\mathrm{kg} / \mathrm{m}^{3}$ \\
\hline$\rho_{\text {center }}$ & mass density at the planetary centre & $\mathrm{kg} / \mathrm{m}^{3}$ \\
\hline$\rho_{\text {transition }}$ & mass density required for phase transition & $\mathrm{kg} / \mathrm{m}^{3}$ \\
\hline$\sigma$ & electrical conductivity & $\Omega^{-1} \mathrm{~m}^{-1}$ \\
\hline$\sigma_{\mathrm{E}}$ & electrical conductivity of the Earth & $\Omega^{-1} \mathrm{~m}^{-1}$ \\
\hline$\sigma_{\mathrm{J}}$ & electrical conductivity of Jupiter & $\Omega^{-1} \mathrm{~m}^{-1}$ \\
\hline$\tau$ & time constant for stellar wind evolution $\left(2.56 \cdot 10^{7} \mathrm{yr}\right)$ & $\mathrm{yr}$ \\
\hline$\tau_{\text {circ }}$ & timescale for tidal circularisation & yr, Myr, Gyr \\
\hline$\tau_{\mathrm{CME}}$ & duration of a CME & s, hours \\
\hline$\tau_{\text {sync }}$ & timescale for tidal locking & yr, Myr, Gyr \\
\hline$\phi_{\mathrm{X}}$ & stellar X-ray flux & $\mathrm{W} / \mathrm{m}^{2}$ \\
\hline$\Phi$ & flux of planetary radio emission & $\mathrm{Jy}, \mathrm{W} \mathrm{m}{ }^{-2} \mathrm{~Hz}^{-1}$ \\
\hline$\Phi_{\mathrm{AU}}$ & flux of planetary radio emission at 1 AU distance & $\mathrm{Jy}, \mathrm{W} \mathrm{m} \mathrm{m}^{-2} \mathrm{~Hz}^{-1}$ \\
\hline$\Phi_{s}$ & flux of planetary radio emission at distance $s$ & $\mathrm{Jy}, \mathrm{W} \mathrm{m}{ }^{-2} \mathrm{~Hz}^{-1}$ \\
\hline$\Phi_{\mathrm{CME}}^{\mathrm{s}}$ & flux of planetary radio emission due to strong CME & $\mathrm{Jy}, \mathrm{W} \mathrm{m}{ }^{-2} \mathrm{~Hz}^{-1}$ \\
\hline$\omega$ & angular frequency of planetary rotation & $\mathrm{s}^{-1}$ \\
\hline$\omega_{\mathrm{E}}$ & rotational angular frequency of the Earth $\left(7.27 \cdot 10^{-5} \mathrm{~s}^{-1}\right)$ & $\mathrm{s}^{-1}$ \\
\hline$\omega_{\mathrm{J}}$ & rotational angular frequency of Jupiter $\left(1.77 \cdot 10^{-4} \mathrm{~s}^{-1}\right)$ & $\mathrm{s}^{-1}$ \\
\hline$\omega_{\star}^{\text {crit }}$ & stellar rotation at which stellar tides become important & $\mathrm{s}^{-1}$ \\
\hline$\omega_{\mathrm{f}}$ & final angular frequency of rotation & $\mathrm{s}^{-1}$ \\
\hline$\omega_{\mathrm{i}}$ & initial angular frequency of rotation & $\mathrm{s}^{-1}$ \\
\hline$\omega_{\text {orbit }}$ & orbital angular frequency & $\mathrm{s}^{-1}$ \\
\hline$\Omega$ & solid angle of radio emission & 1 \\
\hline
\end{tabular}




\section{Abstract}

Since 1995, more than 150 extrasolar planets were detected, of which a considerable fraction orbit their host star at very close distances. Gas giants with orbital distances below 0.1 AU are called "Hot Jupiters". Current detection techniques are not sensitive enough for the detection of Earth-like planets, but such planets are expected at similar orbital positions. For all these so-called close-in extrasolar planets, the interaction between the stellar wind and the planetary magnetosphere is expected to be very different from the situation known from the solar system. Important differences arising from the close substellar distances include a low stellar wind velocity, a high stellar wind density and strong tidal interaction between the planet and the star. This interaction is shown to lead, for example, to a synchronisation of the planetary rotation with its orbit ("tidal locking"). Taking these points into account, planetary magnetic moments are estimated and sizes of planetary magnetospheres are derived. Two different effects resulting from the magnetospheric interaction are studied in detail. (a) Characteristics of radio emission from the magnetospheres of "Hot Jupiters" are discussed. It is shown that the frequency range and the sensitivity of current radio detectors are not sufficient to detect exoplanetary radio emission. With planned improvements of the existing instrumentation and with the construction of new radio arrays, the detection of exoplanetary radio emission will be possible in the near future. (b) The flux of galactic cosmic rays to the atmospheres of terrestrial exoplanets in close orbits around $\mathrm{M}$ stars is studied. Different types of planets are shown to be weakly protected against cosmic rays, which is likely to have implications for planetary habitability. This should be taken into account when selecting targets for the search for biosignatures in the spectra of terrestrial exoplanets. 


\title{
1 Introduction
}

There cannot be more worlds than one.

Aristotle, 384-322 BC

\author{
There are infinite worlds both like \\ and unlike this world of ours. \\ Epicurus, 341-270 BC
}

The discovery of a planet around the star 51 Pegasi (Mayor and Queloz 1995) marks the beginning of a new era for planetary sciences, in which planets around other stars have finally become accessible to observations. In the last ten years, the number of known extrasolar planets (or "exoplanets") has rapidly grown to 170 planets (as of 26 November 2005). With improved instrumentation, many more detections are expected in the near future.

Many extrasolar planets have properties which surprised those who expected them to be similar to the planets of the solar system. For example, many planets were found in highly eccentric orbits (Halbwachs et al. 2005). Furthermore, many Jupiter-mass planets have orbital distances of 0.05 astronomical units or less 11 (Udry et al. 2003). This was especially surprising because the existence of such objects cannot be explained within the standard theory of planet formation (Glanz 1997).

The small orbital distance has important consequences for the planetary nature and evolution. The following three effects directly result from the small orbital distance: First, the increased flux of stellar light strongly heats the planet. Second, the stellar wind density at the planetary orbit is considerably higher than at larger distances. Third, the small orbital distance gives rise to strong tidal interaction between the star and the planet. Each of these primary effects leads to additional, secondary effects. For example, it is known that the intense stellar irradiation of the planet slows down the planetary contraction, leading to a larger radius when compared to a more distant planet of the same age (Burrows et al. 2000, Guillot and Showman 2002). The strong heating also leads to a highly increased atmospheric loss (Vidal-Madjar et al.|2003, Lammer et al.|2003). The high stellar wind density is responsible for a high energy flux into planetary magnetospheres. Finally, tidal interaction results in a modification of the planetary orbital characteristics (orbital distance, eccentricity and inclination). It may also change the planetary rotation period, the most extreme case being that where the planetary rotation period is synchronised with the orbital period.

\footnotetext{
${ }^{1}$ The mean distance between the Earth and the Sun is approximately equal to one astronomical unit (1 AU).
} 
Several of these effects are relevant for the interaction of the planetary magnetosphere with the ambient stellar wind. The importance of these effects has to be evaluated carefully: Does planetary evaporation modify the shape of the magnetosphere? How strong is the compression of the magnetosphere by the dense stellar wind? How does tidal interaction modify the planetary magnetic field?

On the other hand, the resulting changes in the configuration of the planetary magnetosphere are important for different fields of exoplanet studies, including planetary evolution and the interpretation of current observations. In addition, potential new observation techniques are based on such interaction. More specifically, one might raise the following questions: Under what circumstances does the magnetosphere protect the atmosphere from the stellar wind? If not, how strongly does this influence the atmospheric escape? How strong is the magnetospheric radio emission for close-in planets? Does the magnetosphere still shield the planetary atmosphere against high energy particles?

It is the aim of this work to provide answers to some of the above questions. The quantities influencing the interaction of the stellar wind with planetary magnetospheres as well as effects resulting from it are discussed. Fig. 1.1 schematically shows the interrelation between different effects addressed within this work.

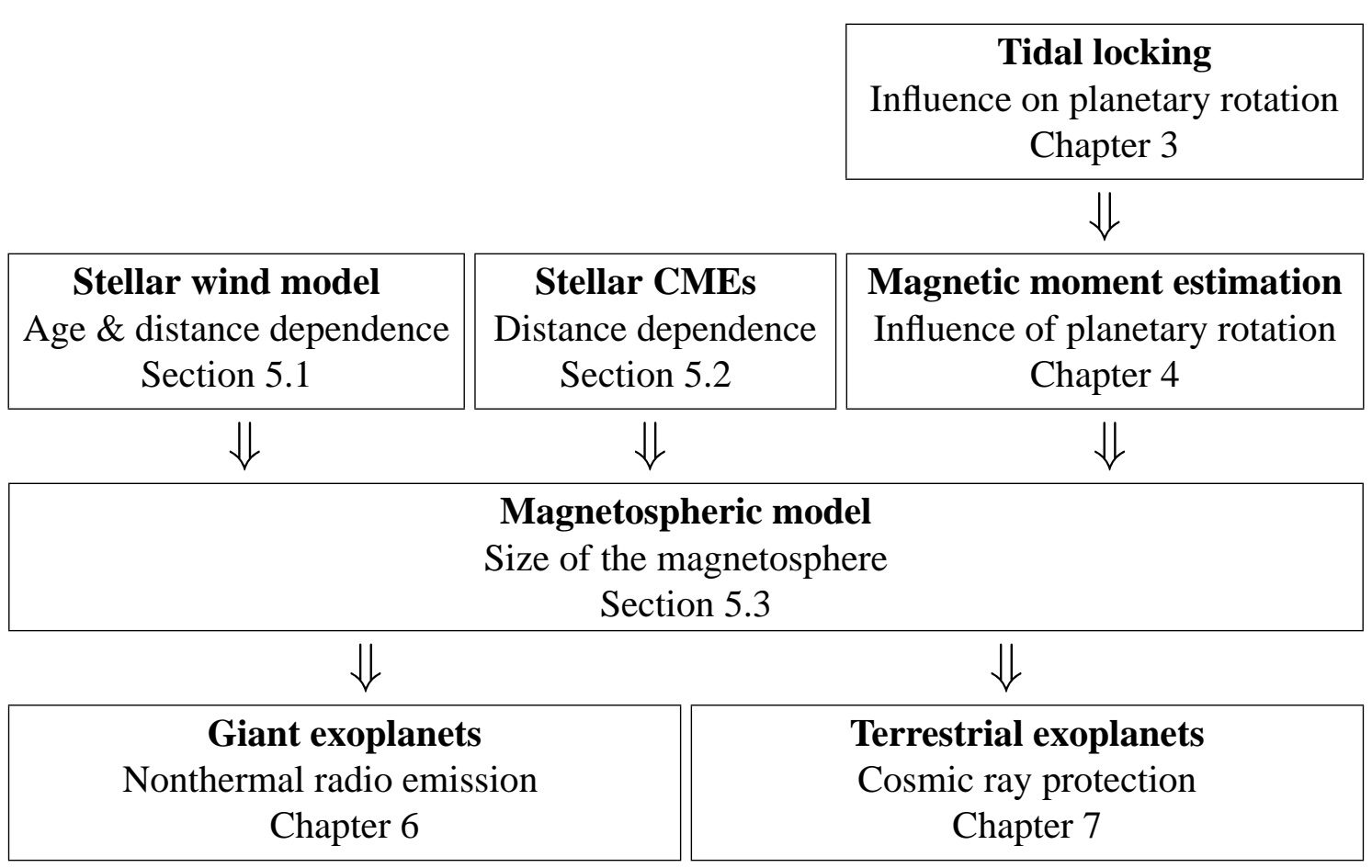

Figure 1.1: Aspects of the interaction of exomagnetospheres with the stellar wind for close-in extrasolar planets.

To familiarise the reader with the background of this work, a short introduction on extrasolar planets is given in Chapter 2. Important concepts are introduced, and the planetary and stellar parameters required in the following sections are presented.

Different aspects of the tidal interaction between the planet and the star are examined in Chapter 3. It is studied how tidal interaction influences the planetary rotation rate 
(Section 3.1), the orbital eccentricity (Section 3.2) and the inclination of the planetary spin axis with respect to the orbit normal (Section 3.3).

In Chapter 4, the influence of tidal interaction on the planetary magnetic moment is discussed. This is done separately for two classes of extrasolar planets, namely for gaseous giant planets and for rocky, terrestrial planets. Different analytical scaling laws for the planetary magnetic moment are compared and evaluated. In the case of gaseous giant planets, the quantities of the planetary core required for the calculation of the magnetic moment are obtained from a hydrostatic planetary model, whereas for terrestrial planets published models of the planetary interior are used.

The magnetosphere is shaped by the interplay between the planetary magnetic field and the stellar wind. For this reason, the stellar wind conditions prevailing at close orbital distances are studied in Section 5.1. In addition, the role of stellar CMEs (as analogues to solar coronal mass ejections) is analysed in Section 5.2. From these input parameters, the size and shape of the corresponding planetary magnetospheres can be obtained. Using a potential field model, the magnetosphere is described in Section 5.3, which thus stands at the centre of this work (see Figure 1.1). With the magnetosphere thus determined, it is possible to estimate the strength of different magnetospheric effects in the following chapters.

One of these results concerns the strength of magnetospheric radio emission from gaseous giant exoplanets. From the solar system, it is known that all strongly magnetised planets are sources of nonthermal radio emission. Different studies suggest that, because of the enhanced stellar wind density, the radio emission from close-in exoplanets may be considerably stronger than that of the planets of the solar system (Farrell et al. 1999, Zarka et al. 2001). This is discussed in detail in Chapter 6. The range of expected emission frequencies and the radio flux received at Earth are calculated within the frame of an analytical model (Section 6.1). The resulting values are compared to the corresponding stellar radio emission (Section 6.2) and to the detection limits of current and planned detectors (Section 6.3).

Although no Earth-sized exoplanets have been detected yet, such a detection is likely to occur in the near future. The Earth's magnetosphere is known to be important for the protection of the planetary surface against the impact of high-energy cosmic ray particles. With the influence of tidal locking on the planetary magnetic moment mentioned above, the question arises whether the same degree of protection can be expected for Earth-like extrasolar planets. This question is studied in Chapter 7. After a short introduction on cosmic rays (Section 7.1), the numerical model used for the calculation of cosmic ray trajectories is outlined (Section 7.2). In Section 7.3, results are given for different planets, studying the influence of tidal locking, stellar system age, and planetary size.

Finally, Chapter 8 concludes by summarising the results obtained within this work. 


\title{
2 Extrasolar planets: An overview
}

\author{
It is not unreasonable that a planet might exist at a distance \\ of 1/50 astronomical unit, or about 3,000,000 km. \\ O. Struve, Observatory $72,199,1952$
}

The search for extrasolar planets can be amazingly rich in surprises. [With] the rather unexpected orbital parameters of 51 Peg b, searches begin to reveal the extraordinary diversity of possible planetary formation sites.

M. Mayor and D. Queloz, Nature 378, 355, 1995

Usually, the discovery of a planet around the star 51 Pegasi (Mayor and Queloz 1995) is considered to be the starting point for the rapidly growing field of extrasolar planet science. Over the past ten years, more than 150 extrasolar planets have been discovered and new observation methods have been developed. Different theories and models have been suggested, some of which were later disproved. Others have been confirmed, and many more have not yet been tested by observations. It is beyond the scope of this work to summarise all knowledge on exoplanets. However, some background information on extrasolar planets is required to embed this work in the right context. For this reason, different areas of exoplanet study are briefly presented in the remainder of this chapter. This includes the history of exoplanet detection (Section 2.1) and some useful definitions (Section 2.2). In Section 2.3, the concept of the habitable zone is introduced, and in Section 2.4 all methods successfully employed to observe extrasolar planets are compared. One method, namely the observation of enhanced chromospheric emission by the star, is discussed in more detail in Section 2.5. Finally, the planetary and stellar parameters which are required in later sections are presented in Section 2.6. Those readers already familiar with extrasolar planets may wish to skip Chapter 2 and continue directly with Chapter 3 .

\subsection{Historical development}

Long before observational methods capable of the detection of other planets became available, mankind had started to speculate about the existence of such objects. Opinions ranged from "There are infinite worlds both like and unlike this world of ours" (Epicurus, 341-270 BC) to "There cannot be more worlds than one" (Aristotle, 384-322 BC), which demonstrates the differences between the extreme positions (Perryman 2000). Shortly before the year 1600, Giordano Bruno and Thomas Digges suggested the existence of planets in orbits around other stars (Trimble 2004). As knowledge on the solar system 
increased, the expectation that planets could also exist around other stars became more common.

In the 20th century, advances in instrumentation finally allowed to begin the search for planets outside the solar system. The first announced detections-like that of a planet around Barnard's star in the year 1963-were later rejected (see, e.g. Lissauer|2002, Trimble 2004), but serve as a proof of the interest taken in that subject.

The first true exoplanet candidate which was detected is HD 114762b. It was discovered in the year 1988 as a binary object to the star HD 114762 (Latham et al.|1989). At that time, it was classified as a "probable brown dwarf". The uncertainty in this formulation results from the fact that only the lower limit of its mass is known. If the true mass of HD $114762 \mathrm{~b}$ is close to this lower limit, it is a planet, while a higher mass would make it a brown dwarf (a more precise definition is given in Section 2.2). According to the terminology used today, the object is a "planetary candidate".

The first objects outside the solar system for which the observations clearly indicated a planetary mass were found around the pulsar PSR 1257+12, which itself had been discovered in 1990 (Wolszczan and Frail 1992). Whether these objects should be called planets or not is a matter of definition (see Section 2.2). In any case, this discovery observationally confirmed the existence of stellar systems radically different from the solar system.

In the year 1995, Mayor and Queloz (1995) found a planet with a minimum mass of half of that of Jupiter at an orbital distance of 0.05 AU around the star 51 Pegasi. With this discovery, the question whether planets around Sun-like stars exist outside the solar system is finally settled.

With this question being answered, many new questions have arisen.

\subsection{Definitions}

Surprisingly, the term "Extrasolar Planet" is not well defined. While it is clear that "extrasolar" denotes an object outside the solar system, at least two different definitions for "planet" are in use. For this reason, certain objects are classified as extrasolar planets in some publications, while in others they are not. To shed some light on the differences, the most common definitions are presented here. Also, two subclasses of planets frequently referred to in this work are defined below.

\subsubsection{Extrasolar planets: working definition of the IAU}

Rather than giving a definitive, detailed definition of the term "planet", the Working Group on Extrasolar Planets (WGEP) of the International Astronomical Union (IAU) developed the following working definitions?

1. "Objects with true masses below the limiting mass for thermonuclear fusion of deuterium (currently calculated to be 13 Jupiter masses for objects of solar metallicity) that orbit stars or stellar remnants are 'planets' (no matter how they formed).

${ }^{1}$ See http://www.ciw.edu/IAU/div3/wgesp/definition.html 
The minimum mass/size required for an extrasolar object to be considered a planet should be the same as that used in our Solar System."

2. "Substellar objects with true masses above the limiting mass for thermonuclear fusion of deuterium are 'brown dwarfs', no matter how they formed nor where they are located."

3. "Free-floating objects in young star clusters with masses below the limiting mass for thermonuclear fusion of deuterium are not 'planets', but are 'sub-brown dwarfs' (or whatever name is most appropriate)."

This definition of "planet" includes pulsar planets (i.e. planets around pulsars), and excludes free floaters. Also, objects orbiting brown dwarfs are not called planets; neither are brown dwarfs orbiting stars. The mass range for brown dwarfs is usually given by $13 M_{\mathrm{J}} \leq M_{\mathrm{bd}} \leq 80 M_{\mathrm{J}}$ (where $M_{\mathrm{J}}$ is the mass of Jupiter). Lighter objects are called either planets or sub-brown dwarfs, and heavier objects are stars.

\subsubsection{Extrasolar planets: alternative definition of G. Basri}

An alternative set of definitions was suggested by G. Basri?

1. A fusor is an object that achieves core fusion during its lifetime.

2. A planemo (short for "planetary mass object") is an object that is spherical due to its own gravity, and that is never capable of core fusion.

3. A planet is a planemo which is formed in orbit around a fusor.

This definition of "planet" excludes pulsar planets and free floaters (except for free floaters for which there is evidence that they were ejected from a planetary system). Objects orbiting brown dwarfs are called planets, but brown dwarfs orbiting stars are not. The advantages and disadvantages of this definition are described by Basri and Brown (2006).

\subsubsection{Extrasolar planets: definition used in this work}

Within this work, objects with masses below the critical mass for nuclear fusion of deuterium in orbits around main sequence stars are studied. According to both definitions given above, such objects qualify as planets. At the same time, this excludes planets around stellar remnants like pulsars, planets around non-main sequence stars, and objects around brown dwarfs. The reason for this limitation is that most currently known exoplanets were found around main sequence stars. An extension of this work to planets around other objects is possible, but it would involve additional and highly different fields of (astro)physics.

\footnotetext{
${ }^{2}$ See e.g. http://astron.berkeley.edu/ basri/defineplanet/Mercury.htm and http://astron.berkeley.edu/ basri/defineplanet/what saplanet.htm
} 


\subsubsection{Hot Jupiters}

Not very long after the discovery of the extrasolar planet around the star 51 Pegasi by Mayor and Queloz (1995), the denomination "Hot Jupiter" was introduced to describe the subclass of gaseous giant planets at small orbital distances (Schilling 1996). Although no precise boundary exists, a planet is usually considered to be a Hot Jupiter when its mass is considerably larger than that of Saturn $\left(M_{\mathrm{S}}=0.3 M_{\mathrm{J}}\right)$ and its orbital distance is smaller than 0.1 AU. Because of the extremely small orbital distances, such planets are heated to very high temperatures ( $\gtrsim 1000 \mathrm{~K}$ ), and the evolution of a Hot Jupiter may differ significantly from that of a comparable planet at larger orbital distances (Collier Cameron 2002). Besides the strong stellar irradiation, one of the special features of these planets is their strong tidal interaction with their host star (see Section 3). This is the reason why these planets are usually treated as a special and separate class of planets.

In this work, Hot Jupiters constitute one of the two planetary classes which are discussed in detail. Hereafter, a planet is considered to be a Hot Jupiter if it is (a) predominantly gaseous in composition, (b) has an orbital distance of less than $0.1 \mathrm{AU}$ and (c) is heavier than Saturn.

\subsubsection{Terrestrial Exoplanets}

Another class of extrasolar planets of special interest for this work is that of "Terrestrial Exoplanets". This term denotes planets similar to the terrestrial planets of the solar system (Mercury, Venus, Earth, Mars). For classification as "terrestrial", not the mass of a planet, but its composition is decisive: terrestrial planets mainly consist of rocks (as opposed to planets mainly composed of gases or ices). Note that in this work especially terrestrial exoplanets around low mass stars ( $\mathrm{K}$ and $\mathrm{M}$ type) are of interest, because for these stars the "habitable zone" (i.e. the range of orbital radii for which liquid water can exist on the planetary surface, see Section 2.3 is located much closer to the star than for solar mass stars, making the detection of potentially habitable planets easier to achieve.

\subsection{The habitable zone}

One of the concepts frequently referred to in this work is that of the so-called "habitable zone". It was introduced to describe whether a planet may or may not be potentially habitable for life.

The question whether other life-bearing planets may exist is one of the oldest questions of mankind. While the search for Earth-sized extrasolar planets is still beyond the capabilities of the currently available instrumentation (see Section 2.6.3), important advances are to be expected in the near future, e.g. with ESA's DARWIN or NASA's TPF-C mission. At the same time, ideas are being developed how planets with an atmosphere favourable for the evolution of life could be observationally discriminated (Kasting 1997, Selsis et al. 2002, Selsis 2004).

To direct such search programs towards planets where life is possible, the concept of the "habitable zone" (frequently abbreviated as HZ) is used. The habitable zone around a star is usually defined as the region in which liquid water can exist on the planetary 
surface. According to the atmospheric model of Kasting et al. (1993), for an Earth-like planet around a Sun-like star, this is the case when

$$
S_{\text {eff,min }} \leq \frac{S_{\star}}{S_{\odot}} \leq S_{\text {eff,max }}
$$

Here, $S_{\star}$ and $S_{\odot}=1360 \mathrm{Wm}^{-2}$ denote the stellar energy flux at the location of the planet, and the solar energy flux at Earth, respectively. $S_{\text {eff,min }}$ and $S_{\text {eff,max }}$ are dimensionless values which give the minimum and maximum allowed energy flux relative to the flux currently reaching the Earth's location. These numbers depend on the spectral type (and thus on the mass) of the star. For the Sun, Kasting et al. (1993) obtain $S_{\text {eff,min }}=0.84$ and $S_{\text {eff,max }}=1.67$. For a stellar flux below the limit imposed by eq. (2.1), the surface temperature drops below $273 \mathrm{~K}$ even for the case where the partial pressure of $\mathrm{CO}_{2}$ is chosen such that the greenhouse effect is maximised ("maximum greenhouse" limit of Kasting et al. 1993). For temperatures below $273 \mathrm{~K}$, water is not liquid anymore? For a smaller $\mathrm{CO}_{2}$ pressure the emitted infrared flux is larger because of the less efficient greenhouse effect, and for a larger $\mathrm{CO}_{2}$ pressure the absorbed stellar flux decreases because of increased Rayleigh scattering. If the stellar flux exceeds the limit given by eq. (2.1), the planetary oceans are completely evaporated because the atmosphere becomes optically thick at infrared wavelengths, which in turn limits the infrared flux from the planet to space ("runaway greenhouse" limit of Kasting et al. 1993). Because the energy flux depends on the stellar luminosity $L_{\star}$ as

$$
S_{\star}=\frac{L_{\star}}{4 \pi d^{2}},
$$

equation (2.1) can be rewritten in terms of the orbital distance $d$. Thus, the limits of the habitable zone are determined by

$$
d_{0} \sqrt{\frac{1}{S_{\text {eff,max }}\left(M_{\star}\right)} \frac{L_{\odot}}{L_{\star}}} \leq d \leq d \sqrt{\frac{1}{S_{\text {eff,min }}\left(M_{\star}\right)} \frac{L_{\odot}}{L_{\star}}}
$$

$L_{\odot}$ and $d_{0}$ are the solar luminosity and the Earth's orbital distance (1 AU), respectively.

These results can be applied to stars of different stellar classes. In this case, both the stellar luminosity and the different stellar spectrum have to be taken into account: low mass stars are cooler, and thus emit a larger portion of their energy in the red part of the visible and the near infrared. In this wavelength range, the diffuse reflection by Rayleigh scattering is much less efficient $\left(\propto \lambda^{-4}\right)$ than in the visible. In addition, the atmospheric constituents $\mathrm{H}_{2} \mathrm{O}$ and $\mathrm{CO}_{2}$ have much stronger absorption coefficients in this wavelength range. Thus, most of the incident energy is deposited on the planet (i.e. the bond albedo is lower than for a Sun-like star). This is the reason why a smaller value of $S_{\text {eff }}$ is sufficient to reach the runaway greenhouse or the maximum greenhouse limit. Correspondingly, for very low mass stars, the limits of the habitable zone are at larger orbital distances when the spectral shape of the stellar emission is taken into account than when this effect is neglected. Figure 2.1 shows the size of the habitable zone obtained in this way (Kasting et al. 1993).

\footnotetext{
${ }^{3}$ In the range of allowed parameters, the melting temperature of water depends only weakly on pressure.
} 


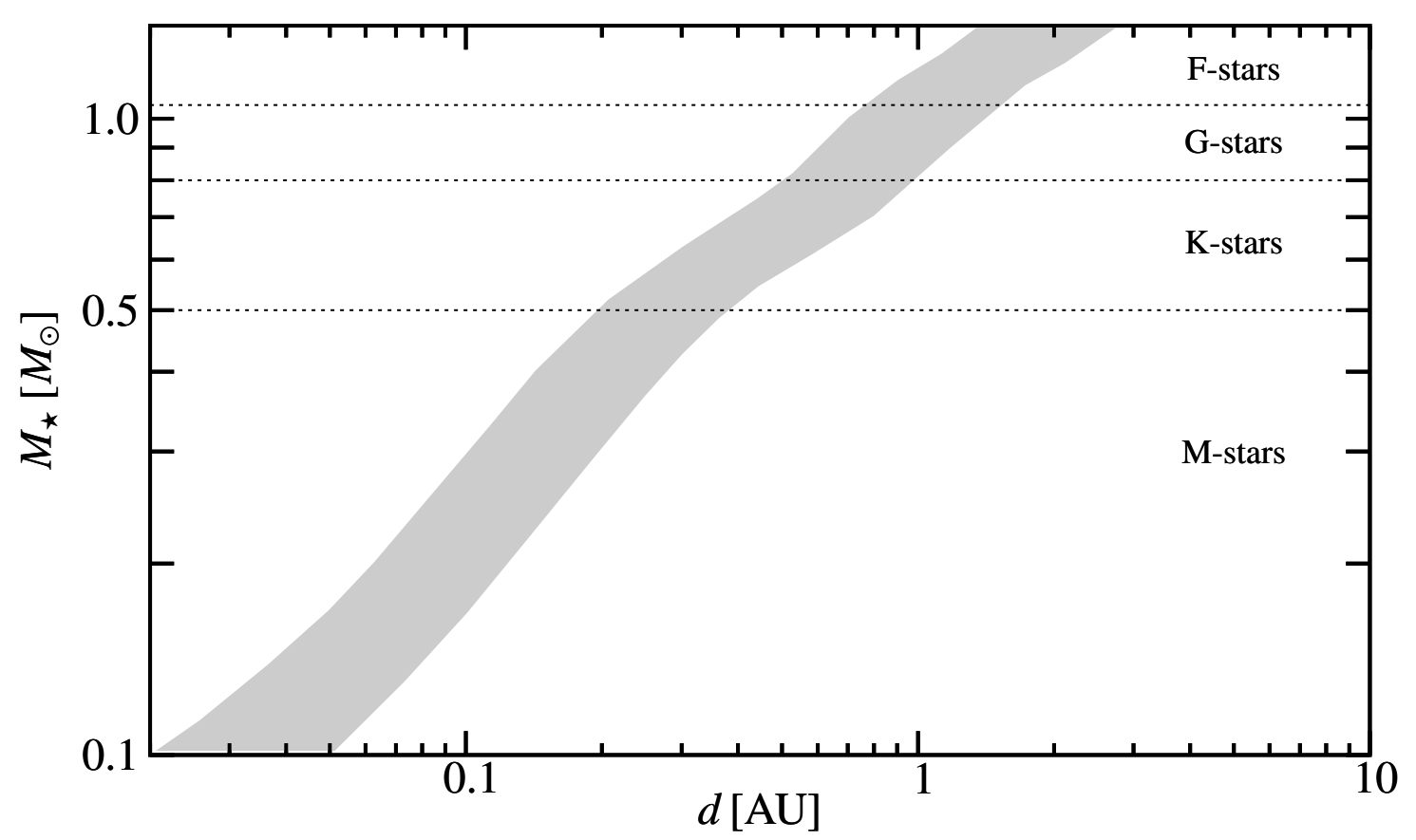

Figure 2.1: Habitable zone with $\tau_{\mathrm{h}}=0$ according to Kasting et al. (1993).

The stellar luminosity $L_{\star}$ and the critical effective fluxes $S_{\text {eff,min }}$ and $S_{\text {eff,max }}$ are not only a function of stellar mass, but also of stellar age. Therefore the location of the habitable zone depends on the stellar age, too. For this reason, different types of habitable zones exist. Kasting et al. (1993) use the concept of the continuously habitable zone (CHZ). It is defined as the zone which is habitable starting at the moment when the star reaches the Zero-Age Main-Sequence. Habitability is required to last at least a certain time $\tau_{\mathrm{h}}$. Thus, in principle, the width of the continuously habitable zone depends on $\tau_{\mathrm{h}}$. For low-mass stars $\left(M_{\star}<0.5 M_{\odot}\right.$, where $M_{\odot}$ denotes the mass of the Sun), however, it turns out that the width of the continuously habitable zone is practically independent of $\tau_{\mathrm{h}}$ as long as $\tau_{\mathrm{h}} \leq 5 \mathrm{Gyr}$ (Kasting et al. 1993, Figures 14 and 15). Stars with larger stellar masses than those shown in Fig. 2.1 $\left(M_{\star}>1.4 M_{\odot}\right)$ have a main sequence lifetime of $t_{\mathrm{ms}}<3 \mathrm{Gyr}$ and are of minor importance concerning habitability.

Note that, strictly speaking, the size and location of the habitable zone does not only depend on the size of the star, but also on the planetary atmospheric composition (Kasting et al. 1993, Joshi et al. 1997), the planetary mass and radius (Kasting et al. 1993, Joshi et al. 1997), the growth rate of the continental area (Franck et al.|2000) and the total continental coverage of the planet (Franck et al.|2003). Furthermore, planetary habitability is probably also influenced by biological activity on the planet (Kasting et al. 1993). Also, the concept of the habitable zone is limited to life as we know it; hypothetical life based on other elements than carbon does not require an environment with liquid water (Bains 2004).

The presence of liquid water is not the only condition necessary for life. Additional factors may be required to make a planet habitable, but are usually not included in the definition of the habitable zone. These factors include (but are not limited to): 
- Obliquity variations: The importance of small obliquity variations with regard to habitability is still debated. One the one hand, some researchers assume that small enough obliquity variations are required for habitability (Atobe et al. 2004); on the other hand, others claim that high obliquity variations are not critical with regard to planetary habitability (Williams and Kasting 1997).

- When the planetary system includes several planets, a dynamically stable orbit is required for long-term habitability.

- A stable atmosphere. Possible causes for the loss of the planetary atmosphere include hydrodynamic escape and ion pick-up. The latter effect is especially relevant for weakly magnetised planets (Khodachenko et al. 2006, Lammer et al. 2006).

- Low enough flux of cosmic rays (Grießmeier et al.|2005b, 2006d). This is especially relevant for weakly magnetised planets.

Because of their relatively small mass, low luminosity and large abundance $(\approx 70 \%$ of the stars in the solar neighbourhood), M dwarfs are frequently suggested as targets for the search for habitable planets. While such planets would be synchronously rotating, the resulting surface temperatures do not exclude planetary habitability (Heath et al. 1999). However, for such planets small magnetic moments are expected, which pose additional constraints to habitability. One of these effects, namely the increased flux of galactic cosmic rays, is described in detail in Section 7 .

\subsection{Overview of current observation methods}

In this section, the different methods currently used to obtain information on extrasolar planets are briefly described. Some methods were used to detect previously unknown planets; with others, such planets were further analysed. The amount of information obtained by various methods strongly differs. While the planetary orbital period can be determined by any of the following methods, some yield a wealth of additional information. To date, the following observation techniques were successfully employed in extrasolar planet studies:

- Pulsar pulse timing: As was described in Section 2.1, the first extrasolar planets were detected around a pulsar, which itself had been detected in 1990. Precise measurements of the radio pulses observed from the millisecond pulsar PSR 1257+12 showed periodic deviations from a constant pulse period (Wolszczan and Frail 1992). This was explained by the presence of planetary companions which lead to a periodic motion of the pulsar around the centre of mass of the planetary system. The varying velocity of the emitter relative to the observer leads to a minute Doppler-shift in the pulse length.

This method is limited to pulsar planets, which are usually regarded as somewhat exotic objects. Most exoplanet research deals with planets around main sequence stars, for which pulse timing it not applicable. 
- Radial velocity: Most of the currently known extrasolar planets were detected with the radial velocity method. Similarly to the pulsar pulse method, it relies on the periodic motion of the star around the common centre of mass. This motion leads to a slight Doppler-shift of the stellar spectral lines. In 1995, the discovery of the planet 51 Pegasi b was achieved using this method (Mayor and Queloz 1995). This discovery is usually viewed as the first detection of a planet around a main sequence star, see Section 2.1.

The radial velocity method requires strong stellar spectral lines, and is especially sensitive towards heavy planets at small orbital distances. Because the inclination $i$ of the planetary orbit with respect to the observer is unknown, this method can only provide information on the projected mass of the planet $M_{\mathrm{p}} \sin i$, plus the orbital radius and eccentricity. On the other hand, the method can be applied to a relatively large number of targets, which currently makes it the most successful tool in the search for extrasolar planets.

- Astrometry: Stars with planetary companions are moving around the centre of mass of the planetary system. The radial velocity method described above measures the component of this motion in the direction towards (or away from) the observer. For close enough stars, the components perpendicular to this axis can be analysed by high precision measurements of the stellar position on the plane of the sky. This was first successfully applied by Benedict et al. (2002).

Because it is possible to observe two velocity components of the stellar motion, astrometry allows to determine the planetary mass without the $\sin i$ ambiguity known from radial velocity measurements (Perryman and Heinaut 2005). Astrometry is currently limited to nearby, low-mass stars with heavy planets, so that only a small number of detections can be expected for the near future. After 2011, the ESA mission Gaia is expected to detect many more ( $\gtrsim 10000)$ planets using astrometry (Mignard 2005).

- Planetary transit: More information can be obtained on the planet when a planetary transit can be deduced from a periodic reduction of the stellar intensity (typically of the order of $1 \%$ ). Because this is only possible for a special viewing geometry $\left(i \approx 90^{\circ}\right)$, only few planetary transits are currently known. This is also one of the reasons why, despite the fact that the transit method has been suggested for a long time (Struve 1952, Rosenblatt 1971), the first transit was observed in the year 1999 (Burrows and Angel 1999, Henry et al.2000, Charbonneau et al.2000)

For transiting planets, the inclination is strongly constrained, so that, in combination with radial velocity measurements, the real mass of the planet $M_{\mathrm{p}}$ can be calculated. In addition, the depth of the transit (i.e. the ratio of observed flux during and out of transit) yields information on the planetary radius. At the same time, the special geometry is the main disadvantage of this technique, because it strongly reduces the number of available targets.

- Transit spectroscopy: During a planetary transit, the reduction of the stellar flux is observed to be enhanced for some spectral lines. At such frequencies, the stellar light is not only blocked by the planetary body, but additional absorption is taking 
place in the planetary atmosphere or exosphere. In this way, the atmosphere of HD 209458b was shown to extend over several planetary radii and to contain $\mathrm{Na}$ (Charbonneau et al.2002), H (Vidal-Madjar et al.2003), as well as C and O (VidalMadjar et al. 2004). Other atmospheric components like $\mathrm{He}, \mathrm{Li}, \mathrm{Fe}$ and $\mathrm{Ca}$ were searched for, but were not yet detected (Moutou et al. 2003, Narita et al. 2005).

The detection of planetary constituents is required for models concerning the planetary composition and atmosphere. Similarly, non-detections provide important upper limits.

- Secondary transit: The observed flux does not only decrease during a primary planetary transit (see above), but also during secondary transits (i.e. when the planet is passing behind the star instead of in front). The reason is that the planetary black-body emission is then blocked by the star. The non-detection of a signal at a characteristic frequency of methane near $3.6 \mu \mathrm{m}$ during the first attempt to observe secondary eclipses helped to constrain atmospheric models (Richardson et al. 2003). More recently, a reduction in the infrared flux of the order of $0.25 \%$ was observed for the planets HD 209458b (Deming et al. 2005) and TrES-1b (Charbonneau et al. 2005).

Single-frequency measurements of the infrared flux allow to determine the effective temperature of the planet; with multi-frequency observations, the infrared part of the planetary spectrum could be analysed. With the currently available data it is already possible to show that the reradiation of the absorbed stellar flux is more likely to occur over the entire planet rather than over one hemisphere only. In addition, first constraints for models of the planetary atmosphere of TrES-1b were derived from the infrared flux at $4.5 \mu \mathrm{m}$ and at $8.0 \mu \mathrm{m}$ (Fortney et al. 2005).

- Microlensing: Gravitational lensing is one of the applications of general relativity theory. When a massive object (the "lensing object") is placed between a distant light source and an observer, the light is bent around the lensing object. This changes the shape of the distant light source; in the case of a point source, the measured intensity is modified by the presence of the lensing object. This effect can be used for the detection of extrasolar planets when the lensing object is a foreground star with a planetary companion. As this star is passing through the line of sight to the distant light source, the asymmetry in the mass distribution caused by the planet leads to additional fine structure of the observed light curve (Mao and Paczyński 1991). In 2003, the object OGLE 2003-BLG-235/MOA 2003-BLG-53 was detected using this method. This is the first confirmed detection of an extrasolar planet using microlensing (Bond et al. 2004).

In principle, a lot of information is hidden in the microlensing light curve, including the planetary mass and its orbital distance. However, one should note that different combinations of stellar and planetary parameters can lead to almost identical lightcurves. Together with the expected stellar variability, it is usually not possible to deduce unequivocal information (Gaudi and Han 2004). In addition, measurements during a microlensing event are not repeatable after the lensing event is terminated. For this reason, microlensing should, whenever possible, not be used alone, but should be combined with other methods. 
- Direct observation: In a few special cases, direct observations of stellar companions were possible using adaptive optics. The first observation was announced by Chauvin et al. (2004), who were able to distinguish the central object and a distant (55 AU) companion on CCD images. Later observations (Chauvin et al. 2005a) showed that both objects were moving across the sky together, which confirmed that the secondary object was not a background star, but a companion. The observations were performed in the infrared, where the contrast between the star and the planet is more favourable (less stellar flux and higher planetary flux than, for example, in the visible).

So far, no direct observations exist for extrasolar planets as defined by the IAU (see Section 2.2.1). In some cases, the central object is a brown dwarf (Chauvin et al. 2004), in other cases, the secondary object is too heavy to be classified as a planet (Chauvin et al.2005b). However, the direct observation of extrasolar planets seems to be merely a question of time. The method is limited to large and hot (i.e. young) planets at large orbital distances around small stars.

- Other potential methods: Besides the methods already used today, a certain number of other methods have been suggested. It is likely that some of these will contribute to the increase of knowledge about extrasolar planets in the future. The list of potential future methods includes

- the spectroscopic detection of stellar light reflected by the planetary atmosphere,

- the photometric detection of stellar light reflected by the planetary atmosphere,

- infrared observation of the planetary system, in which the stellar light is suppressed by interferometry (as planned for the DARWIN mission),

- observations of the planetary system, in which the stellar light is removed by a coronograph (as planned for the TPC-C mission),

- the analysis of enhanced stellar chromospheric emission caused by a close-in planet (which is discussed in Section 2.5)

- and the detection of planetary radio emission (which is discussed in more detail in Chapter 6).

All currently successful observation methods are summarised in Table 2.1. More detailed overviews on different methods are given by Perryman (2000) and Charbonneau (2004). The limits of current and future ground- and space-based observations are described in the report by the ESA-ESO Working Group on Extra-Solar Planets (Perryman and Heinaut 2005). Information on the different ongoing projects can also be found at the Extrasolar Planets Encyclopedia at http: / /www. obspm. fr/encycl/searches.html.

\subsection{Chromospheric heating}

This section describes several additional effects which may be used to obtain information on extrasolar planets. These effects all rely on the heating of the stellar chromosphere 


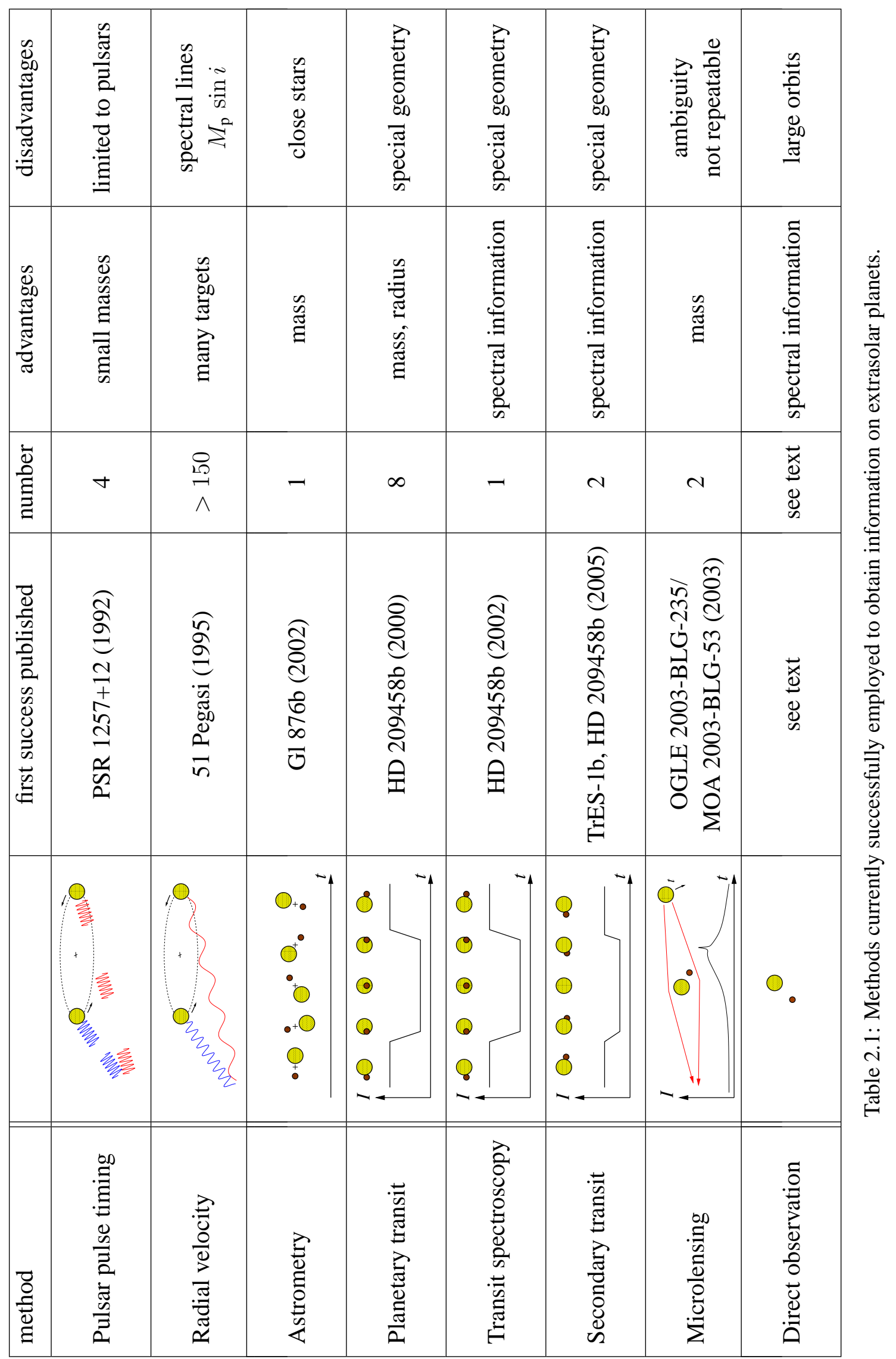


by the interaction of a close-in planet with its host star. This heating enhances the stellar chromospheric emission in different frequency bands, making this interaction accessible to observation and analysis. The status of these observation methods lies somewhere between "potential future method" and "currently employed method". Also, the measurement of such stellar activity is sometimes interpreted as a tentative proof for the existence of planetary magnetic fields, which would be highly relevant for this work. In this section, this interpretation is analysed critically.

Because of the close proximity of Hot Jupiters to their host stars, different modes of interaction may lead to observable effects in the stellar chromosphere. The acceleration of the stellar rotation due to the strong tidal interaction with the planet may lead to increased dynamo field generation and magnetic heating (Saar and Cuntz 2001). Cuntz et al. (2000) suggest that both the creation of a large tidal bulge on the star as well as magnetic interaction between the star and the planet may enhance the activity in the outer stellar regions (i.e. chromosphere, transition region and corona). For tidal interaction (which could lead to enhanced generation of either acoustic or magnetic energy by increasing the turbulent velocity), the relative effect depends on the orbital radius as $d^{-3}$, whereas for magnetic interaction the amplitude of the effect varies as $d^{-2}$. Another major difference between the two models is the number of maxima of the chromospheric emission during one planetary orbit: For tidal interaction, two maxima are expected, but for magnetic interaction, only one maximum should be observable (Cuntz and Shkolnik 2002).

First studies of observational data used one line of the Ca II infrared triplet at $8662 \AA$. Seven planet-hosting stars were observed, but no identification of a planetary signature in the stellar emission was possible (Saar and Cuntz 2001). Subsequent observations in the optical resonance lines of $\mathrm{Ca}$ II $\mathrm{H}$ and $\mathrm{K}$ at 3933 and $3968 \AA$ were more successful and showed signs of an increase of the chromospheric emission of about 1-2\% caused by planets around the stars HD 179949 (Shkolnik et al. 2003, 2004) and $v$ And (Shkolnik et al. 2005). The observations indicate one maximum per planetary orbit, a "Hot Spot" in the stellar chromosphere which precedes the calculated planetary passage (the subplanetary point) by 0.17 and 0.47 in phase, corresponding to a lead angle of $60^{\circ}$ and $169^{\circ}$, respectively. The existence of a non-zero lead angle seems reasonable, because the field lines between the star and the planet are not be straight, but bent.

The exact mechanism responsible for the observed chromospheric heating is not yet determined. Two main models were suggested. In the first model, both the star and the planet are magnetised. Depending on the orientation of the magnetic fields, reconnection might occur between stellar and planetary fieldlines (Ip et al. 2004), thereby creating hot plasma. In analogy to solar flare events (Ip et al. 2004, and references therein) or to the interaction of Jupiter with Io (Shkolnik et al. 2005), the plasma then travels along the magnetic field lines down to the footpoints, constituting an additional source of heat in the stellar chromosphere. If this scenario is true, these observations would constitute the first observational indication for extrasolar magnetospheres. This mechanism would also be consistent with that suggested for the strongest known stellar flares ${ }^{4}$. In the second

\footnotetext{
${ }^{4}$ Some very large flares (up to $10^{7}$ times more energetic than the largest solar flare) were observed for normal F and G stars on, or very close to, the main sequence (Schaefer et al. 2000). Rubenstein and Schaefer (2000) suggest that these "superflares" are caused by magnetic interaction between the star and a (yet undetected) magnetised Hot Jupiter. However, so far only nine of these transient extreme events were detected (Schaefer et al.2000), and firm conclusions are not yet possible.
} 
model, a non-magnetised planet could act as a unipolar inductor like Io around Jupiter, and still cause reconnection (Saar et al. 2004). While the observations seem to indicate that the source of the emission is close to the stellar surface, this would also be possible for a unipolar inductor (Saar et al. 2004). In a more detailed version of this model, the unipolar inductor is replaced by an Alfvén-wing model. The lead angles observed by Shkolnik et al. (2003) and Shkolnik et al. (2005) were recently explained with such a model using realistic stellar wind parameters obtained from the stellar wind model by Weber and Davis (Preusse 2006). This indicates that a magnetised planet is not required to describe the present data.

As long as it is not possible to distinguish between both scenarios (reconnection between star and magnetised planet vs. plasma flow along Alfvén-wings), enhanced chromospheric emission cannot be regarded as a proof for exoplanetary magnetospheres.

\subsection{Planetary parameters}

Since 1995, more than 150 exoplanets were detected 5 , many of them in close orbits around $\mathrm{G}$ and $\mathrm{K}$ stars. In this section, relevant information on the state of detections concerning Hot Jupiters and terrestrial exoplanets is summarised, and some planetary parameters are provided for later use.

\subsubsection{Presently known Hot Jupiters}

Currently, 40 planets with orbital distances of less than 0.1 AU are known. Of these, 11 have minimum masses below $0.3 M_{\mathrm{J}}$. The remaining 29 planets are potential Hot Jupiters. Unfortunately, only for a few planets both mass and radius are known, which are both needed to obtain information on the planetary structure. The mass is required to determine whether the stellar companion is a planet or a brown dwarf, see Section 2.2. For a given planetary mass, the radius is determined by its composition. The largest planetary radius can be achieved by gas spheres composed of hydrogen. For other gases (e.g. helium), the maximum radius is strongly reduced (see Section 4.3.1). For planets composed of ices or rocks, the radius (for a given planetary mass) is again considerably smaller (Guillot et al. 1996).

For the planets HD 209458b, OGLE-TR-10b, OGLE-TR-56b, OGLE-TR-111b, OGLE-TR-113b, OGLE-TR-132b and TrES-1b, the measurements of the planetary radii show hydrogen to be the main constituent. Thus, at least seven "Hot Jupiters" (as defined in Section 2.2.4) are known today 6 .

For all other planets, the radius is not known. However, while gaseous giants of approximately one Jupiter mass are consistent with various planet formation scenarios, the existence of Jupiter-mass terrestrial planets seems unlikely. For this reason, usually all Jupiter-mass planets in close orbits are denoted as Hot Jupiters. Nevertheless, this

\footnotetext{
${ }^{5}$ Presently, 170 planets are known (26 November 2005). The current state can be found e.g. at the Extrasolar Planets Encyclopaedia at http: / /www.obspm.fr/encycl/encycl.html

6 The transiting planet around HD 149026 is not considered as a Hot Jupiter, because it is substantially enriched in heavy elements. Sato et al. (2005) show that the majority of the planetary mass is probably concentrated within a heavy core.
} 
work will (with one exception, namely $\tau$ Bootes) focus on the "confirmed" Hot Jupiters mentioned above, because the planetary radius is required for the calculation of the tidal locking timescale as well as for the models used to estimate the planetary magnetic dipole moment.

\subsubsection{Parameters for Hot Jupiters}

For the calculation of the tidal locking timescale (Section 3.1.3), for the estimation of the planetary magnetic moment (Section 4.3), for the evaluation of the planetary stellar wind environment (Section 5.1.4), and for the calculation of the radio flux (Section 6.1) of Hot Jupiters (as defined in Section 2.2.4), various stellar and planetary values are required. The required stellar parameters include: the stellar radius $R_{\star}$, the stellar mass $M_{\star}$, the stellar age $t_{\star}$, and the distance $s$ to the solar system. As for the planetary parameters, the following values are needed: the planetary radius $R_{\mathrm{p}}$, the planetary mass $M_{\mathrm{p}}$, the orbital frequency $\omega_{\text {orbit }}$ and the radius $d$ of the planetary orbit. In this section, numbers are given for the stellar and planetary parameters.

Of the planetary values, the orbital frequency $\omega_{\text {orbit }}$ is the easiest one to measure. It can be obtained by various techniques: by radial velocity measurement, astrometric observation, transit detection, and observation in the infrared during a secondary transit. All these methods yield periodic signals, from which the orbital frequency can be determined very accurately. Once the orbital frequency (and the stellar mass) is known, the orbital radius $d$ can be calculated from Kepler's third law. For the mass $M_{\mathrm{p}}$, projection effects due to the unknown inclination $i$ of the planetary system limit most observation methods. For radial velocity measurements, for example, only the product $M_{\mathrm{p}} \sin (i)$ can be determined. Usually, two complementary methods have to be employed to obtain the planetary mass $M_{\mathrm{p}}$. For example, if the inclination is known from detected transits, it is possible to calculate the planetary mass from radial velocity data. Similarly, astrometric observations can be combined with radial velocity measurements. The planetary radius $R_{\mathrm{p}}$ is even more difficult to obtain. Currently, it can only be determined from planetary transits, where the depth of the dip in the light curve can be used to estimate the relative radii of the star and the plane ${ }^{7}$. See Section 2.4 for a comparison of the different observation methods.

In this work, only Hot Jupiters for which the radius is either known from transit observations or is reasonably well constrained by theoretical models ( $\tau$ Bootes b) are treated. This restriction is applied because information on the planetary radius is required in the following sections. For such planets, the observational data are summarised in Table 2.2. They were obtained in the following way:

- For HD 209458 (for which the first planetary transit was observed), the existence of a planetary companion was known from radial velocity measurements when the first transit observations were reported by Henry et al. (2000) and Charbonneau et al. (2000). From these measurements, the composition of an extrasolar planet could be deduced for the first time. It was shown that the planet HD 209458b is

${ }^{7}$ The observed "transit radius" is not exactly identical to the standard 1 bar radius (Burrows et al. 2003, 2004a). The differences, however, are of the order of about 5-10\% (depending on the mass of the planet). For this reason, the radii obtained from transit observations will be taken to be equal to the planetary radii in this work. 
a gas giant with hydrogen as its main constituent (Burrows et al. 2000). Here, the more recent planetary data of Cody and Sasselov (2002) are adopted.

- For OGLE-TR-10b, for a long time, it was not clear whether the observed photometric signal was caused by a planet. Accordingly, early publications spoke of a "possible exoplanet" (Konacki et al. 2003b, Bouchy et al. 2005). Recent observations, however, were able to confirm the planetary nature of the companion to OGLE-TR-10 (Konacki et al. 2005). In this work, the parameters of Konacki et al. (2005) are used. These values are based on a combination of additional observations with the observational data of Bouchy et al. (2005).

- For OGLE-TR-56b (the first planet detected by transits), transits were first reported by Konacki et al. (2003a). Here, the planetary values from Bouchy et al. (2005) are used.

- The values for OGLE-TR-111b are taken from Pont et al.(2004). It is the exoplanet with the lowest mass discussed in this work.

- Transits of the planet OGLE-TR-113b were first analysed by Bouchy et al. (2004) and Konacki et al. (2004). Combining the data of these publications, Konacki et al. (2005) derived the planetary parameters with higher accuracy. These improved values are adopted in this work.

- For OGLE-TR-132b, a planetary transit was first announced by Bouchy et al. (2004). Here, the values found by the follow-up observation (Moutou et al.2004) are taken.

- TrES-1b is the first transiting planet detected by a multisite transiting planet survey (Alonso et al. 2004). Using improved estimates of the stellar parameters, the transit data were reanalysed and the planetary parameters were calculated with higher precision by Sozzetti et al. (2004). The latter values are used in this work.

- The inclination of the orbit of the planet around $\tau$ Bootes relative to an observer in the solar system does not allow the detection of planetary transits. For this reason, only the lower limit of the mass $M_{\mathrm{p}}$ of $\tau$ Bootes $\mathrm{b}$ is known from radial velocity measurements $\left(M_{\mathrm{p}} \sin i=4.38 M_{\mathrm{J}}\right.$, Leigh et al. 2003), and the radius is presently not accessible to measurements. Theoretical models by Burrows et al. (2000) yield an upper limit for the radius. This upper limit is $1.58 R_{\mathrm{J}}$ for a planetary mass of $7 M_{\mathrm{J}}$ and $1.48 R_{\mathrm{J}}$ for $10 M_{\mathrm{J}}$. The most probable radius seems to be $1.2 R_{\mathrm{J}}$ (Leigh et al. 2003). Because of this uncertainty, three different models are considered for $\tau$ Bootes b:

- a relatively "light" planet $\left(M_{\mathrm{p}}=4.4 M_{\mathrm{J}}\right.$ and $\left.R_{\mathrm{p}}=1.2 R_{\mathrm{J}}\right)$,

- a "medium" planet $\left(M_{\mathrm{p}}=7.0 M_{\mathrm{J}}\right.$ and $\left.R_{\mathrm{p}}=1.58 R_{\mathrm{J}}\right)$,

- and a "heavy" planet $\left(M_{\mathrm{p}}=10.0 M_{\mathrm{J}}\right.$ and $\left.R_{\mathrm{p}}=1.48 R_{\mathrm{J}}\right)$.

Table 2.2 lists the values of the observed planetary and stellar parameters. The stellar radius $R_{\star}$ and mass $M_{\star}$ are given in units of the solar values $R_{\odot}$ and $M_{\odot}$. The stellar ages are given in $\operatorname{Gyr}\left(1 \mathrm{Gyr}=10^{9}\right.$ years). Note, however, that the determined stellar ages are 
model dependent, and different models usually yield different stellar ages. For this reason, uncertainties of 50\% are not uncommon (Saffe et al. 2005). The distance of the stellar system $s$, when known, is given in pc. The planetary radius $R_{\mathrm{p}}$ and mass $M_{\mathrm{p}}$ are given in units of the respective values of Jupiter (denoted by subscript J), with $R_{\mathrm{J}}=71492 \mathrm{~km}$ (Cain et al. 1995) and $M_{\mathrm{J}}=1.9 \cdot 10^{27} \mathrm{~kg}$ (Raith 1997). Another important quantity is the planetary rotation rate $\omega$. Later, it will be shown that for close-in planets, tidal locking may occur under certain circumstances. In this case, the planetary rotation frequency $\omega$ is given by the orbital frequency $\omega_{\text {orbit }}$. For this reason, Table 2.2 contains the orbital frequency of the planets, normalised to Jupiter's rotation frequency $\omega_{\mathrm{J}}=1.77 \cdot 10^{-4} \mathrm{~s}^{-1}$ (Raith 1997). For Jupiter and Saturn, the planetary rotation rate $\omega$ is given directly by observations. Finally, the orbital distance $d$ is given in AU.

\subsubsection{Presently known terrestrial exoplanets}

While currently over 150 exoplanets are known, Earth-like planets (as defined in Section 2.2.5) outside the solar system are not yet accessible to current detection techniques. The smallest planet detected until recently has a projected mass of $14 M_{\mathrm{E}}$, where $M_{\mathrm{E}}$ is the mass of the Earth (Santos et al. 2004). It orbits the star $\mu$ Arae (HD 160691), but until now its composition could not be determined. Recently, the detection of a planet with $\sim 7.5 M_{\mathrm{E}}$ orbiting the M star GJ 876 was announced by Rivera et al. (2005). Neither a small gas planet, nor a rocky planet can be ruled out for these planets (an icy planet is excluded because of its small orbital distance and the resulting high equilibrium temperature). However, this situation is expected to improve in the near future, when the detection of terrestrial exoplanets will be possible with the transit missions CoRoT (CNES, launch scheduled for 2006) and Kepler (NASA, launch scheduled for 2007). According to Bordé et al. (2003), CoRoT is sensitive enough to allow the detection of exoplanets within the habitable zone of $\mathrm{K}$ and $\mathrm{M}$ dwarfs under the condition that their radius is at least twice that of the Earth. The detection of Earth-size exoplanets orbiting solar-like stars will be possible with Kepler (Jenkins 2002). With the simultaneous measurement of the planetary mass and radius provided by transit detections, it will be possible to deduce constraints for the planetary structure. Additional detections can be expected from the ESA mission Gaia (launch planned in 2011) which will combine precise astrometric measurements with photometric observations (Mignard 2005). Further analysis will be possible with missions like DARWIN (Fridlund 2004), for which launch is planned in 2015 (Perryman and Heinaut 2005).

\subsubsection{Parameters for terrestrial exoplanets}

For terrestrial exoplanets (as defined in Section 2.2.5), the planetary mass $M_{\mathrm{p}}$ and radius $R_{\mathrm{p}}$ are required for later calculations (i.e. the estimation of the tidal locking timescale in Section 3.1.5, and the evaluation of the planetary magnetic moment in Section 4.5). In this section, these parameters are presented.

Within this work, only terrestrial planets for which structure models exist will be studied, treating the orbital distance of the planet and the mass of its host star as free parameters. The following model planets will be analysed: 


\begin{tabular}{|c|c|c|c|c|c|c|c|c|c|c|c|c|}
\hline$\checkmark$ 导 & 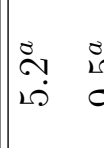 & ip & $\begin{array}{l}\stackrel{i}{i p} \\
\stackrel{i n}{0} \\
0\end{array}$ & $\begin{array}{l}\stackrel{0}{0} \\
\stackrel{H}{*} \\
\stackrel{0}{0}\end{array}$ & $\begin{array}{l}\tilde{i} / \tilde{N} \\
\stackrel{N}{N} \\
\stackrel{0}{0}\end{array}$ & 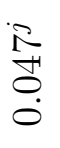 & 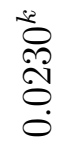 & 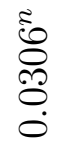 & $\begin{array}{l}\text { के } \\
\text { ळे } \\
\stackrel{0}{0} \\
0\end{array}$ & 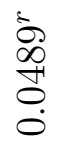 & \begin{tabular}{l}
$\delta$ \\
\multirow{\infty}{\infty}{} \\
$\stackrel{+}{0}$ \\
$\dot{0}$
\end{tabular} & 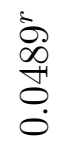 \\
\hline $\begin{array}{cc}5 \\
3 \\
3 \\
3\end{array}$ & 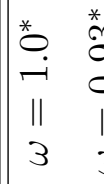 & $\begin{array}{l}n_{0}^{*} \\
\vdots \\
0 \\
0 \\
11 \\
3\end{array}$ & $\stackrel{ن}{\sim}$ & $\stackrel{\stackrel{m}{\longrightarrow}}{\ddot{0}}$ & ڤे & ?. & 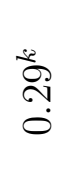 & 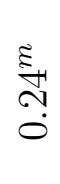 & $\stackrel{2}{\overrightarrow{4}}$ & $\stackrel{\grave{a}}{\stackrel{0}{0}}$ & సેّ & 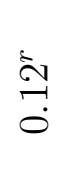 \\
\hline$\Sigma \Xi$ & 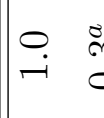 & 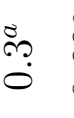 & $\begin{array}{l}\ddot{g} \\
\ddot{0}\end{array}$ & $\begin{array}{l}\tilde{N} \\
\stackrel{1}{2} \\
0\end{array}$ & $\stackrel{\infty}{\stackrel{\infty}{-}}$ & कृ & 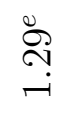 & 芯 & $\begin{array}{l}0 \\
\stackrel{0}{0} \\
0 \\
0\end{array}$ & $\stackrel{\infty}{+}$ & $\stackrel{\infty}{\infty}$ & $\stackrel{\infty}{\circ}$ \\
\hline$\approx \vec{\Xi}$ & $\stackrel{0}{-}$ & $\begin{array}{l}\mathscr{H}_{1} \\
\infty \\
0 \\
0\end{array}$ & $\stackrel{\stackrel{\leftrightarrow}{\sharp}}{\rightarrow}$ & $\stackrel{\stackrel{\sim}{\sim}}{\sim}$ & ํํำ & - & 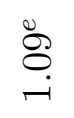 & 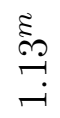 & 范 & $\stackrel{\text { ڤn }}{-}$ & $\begin{array}{l}\infty \\
2 \\
10 \\
-1 \\
-1\end{array}$ & $\stackrel{\infty}{\stackrel{\infty}{+}}$ \\
\hline 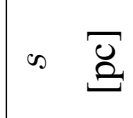 & ' & 1 & 華 & & $\begin{array}{l}50 \\
8 \\
8 \\
10 \\
\end{array}$ & & $\underset{\infty}{\vec{p}}$ & 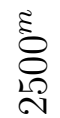 & 总 & ف․ & : & 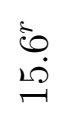 \\
\hline$+^{*}$ 胥 & 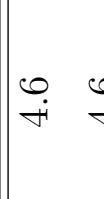 & $\begin{array}{l}0 \\
\dot{\forall}\end{array}$ & 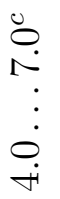 & & $\begin{array}{l}8 \\
0 \\
\dot{H} \\
+1 \\
0 \\
\dot{\infty}\end{array}$ & & & 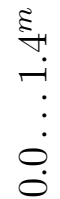 & 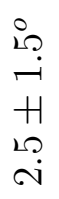 & $\begin{array}{l}0 \\
0 \\
0 \\
H \\
0 \\
0 \\
\dot{H}\end{array}$ & $\begin{array}{l}0 \\
0 \\
0 \\
0 \\
H \\
0 \\
0 \\
-1\end{array}$ & $\begin{array}{l}0 \\
0 \\
0 \\
0 \\
H \\
0 \\
0\end{array}$ \\
\hline$\stackrel{\Xi}{*}^{\Xi}$ & $\stackrel{0}{\circ}$ & $\stackrel{0}{-}$ & نُ & $\stackrel{\mathscr{0}}{\circ}$ & 总 & $\begin{array}{l}\text { î̀ } \\
\infty \\
0 \\
0\end{array}$ & 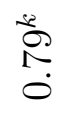 & 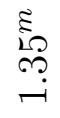 & $\begin{array}{l}\stackrel{8}{\infty} \\
\infty \\
0\end{array}$ & 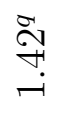 & 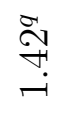 & $\stackrel{\stackrel{\sim}{7}}{\underset{\sim}{\sim}}$ \\
\hline$\underbrace{*} \underset{0}{\square}$ & 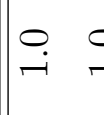 & $\stackrel{0}{-}$ & $\begin{array}{l}\stackrel{0}{\infty} \\
\stackrel{\sim}{ت}\end{array}$ & $\stackrel{8}{\stackrel{-}{0}}$ & ન્ન & $\begin{array}{l}10 \\
\infty \\
0 \\
0\end{array}$ & $\begin{array}{l}\infty \\
\infty \\
1 \\
0 \\
0\end{array}$ & 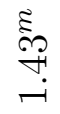 & $\begin{array}{l}\stackrel{\infty}{\infty} \\
\dot{\infty} \\
\dot{0}\end{array}$ & $\stackrel{\infty}{\stackrel{\infty}{+}}$ & $\stackrel{\infty}{\stackrel{\infty}{+}}$ & $\stackrel{\infty}{\stackrel{\infty}{+}}$ \\
\hline 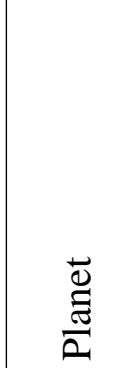 & 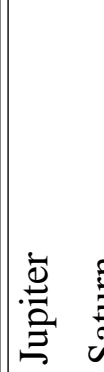 & 苐 & 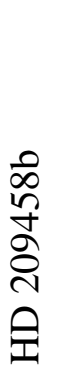 & 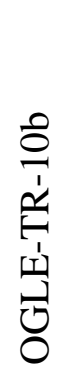 & 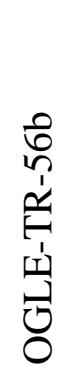 & 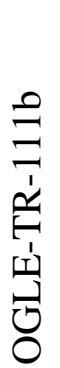 & 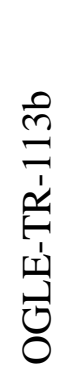 & 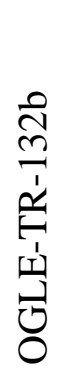 & 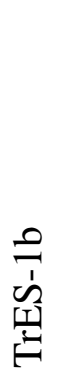 & 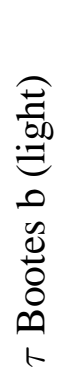 & 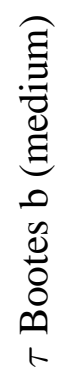 & 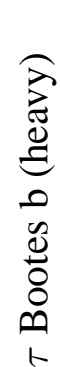 \\
\hline
\end{tabular}

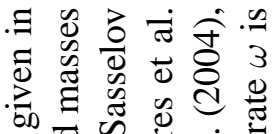

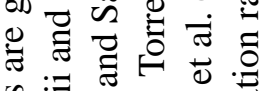

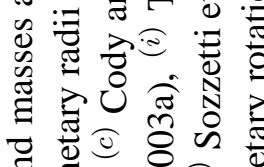
च : $\frac{\pi}{2}$ 久ू

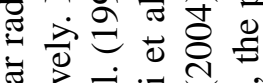

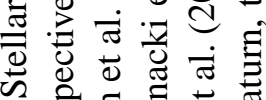
它芯

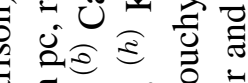

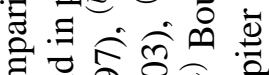

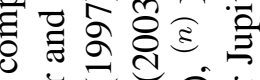

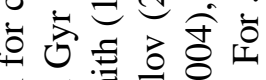

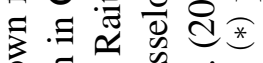
की

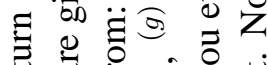
密

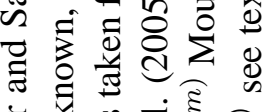
के

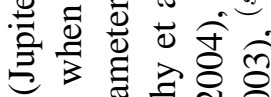

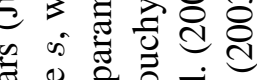

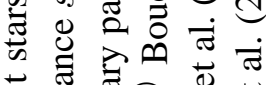

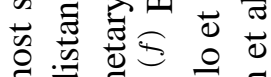

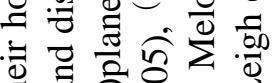
ఖ ज 证希

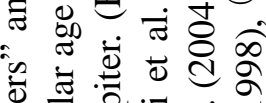

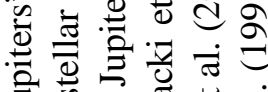

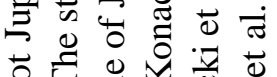

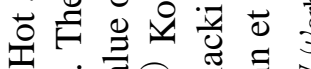

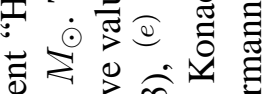

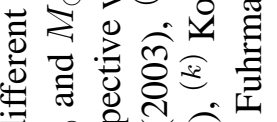
t)

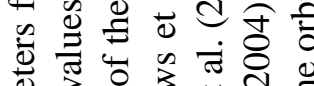

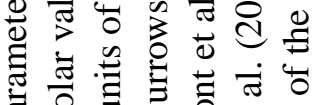
预 ن i

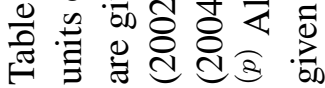


- First, exact Earth analogues will be studied in environments different from that of the Earth. The required parameters of the planet Earth are taken from Cain et al. (1995).

- Within the same frame, smaller planets will be studied. The planet Mercury will serve as an example for small, terrestrial planets. Again, the parameters are taken from Cain et al. (1995).

- As an example for larger planets, a 6 Earth-mass planet (composed of $2 M_{\mathrm{E}}$ metals and $4 M_{\mathrm{E}}$ silicates) is studied. A simple model for the internal structure of such a planet was presented by Léger et al. (2004), in which the silicates form a thick mantle above the metallic core.

- Léger et al. (2004) suggested the existence of so-called "Ocean planets", i.e. large terrestrial planets with a large mass fraction of water $\left(6 M_{\mathrm{E}}, 1 M_{\mathrm{E}}\right.$ of which are metals, $2 M_{\mathrm{E}}$ silicates, and $3 M_{\mathrm{E}}$ ices). The internal structure consists of the metallic core, the silicate mantle, a thick layer of ice, and a liquid water ocean (with a depth of 40 to $133 \mathrm{~km}$, depending, for example, on the surface temperature). Such a planet would be especially interesting because of its larger radius, which makes detection easier.

Table 2.3 lists the planetary parameters for these model planets. Since no terrestrial exoplanets have been detected yet, the parameters cannot be taken from observations. Instead, reasonable parameters have to be assumed. It is especially interesting to study terrestrial exoplanets within the habitable zone (as defined in Section 2.3) of $\mathrm{K}$ and $\mathrm{M}$ stars. For this reason, a stellar mass of $M_{\star}=0.5 M_{\odot}$ is chosen, which corresponds to the border between $\mathrm{K}$ and $\mathrm{M}$ stars. The radius of such a star is $R_{\star}=0.46 R_{\odot}$ (calculated according to Tout et al. 1996). The planetary mass $M_{\mathrm{p}}$ and radius $R_{\mathrm{p}}$ are taken from planetary models. They are given in units of the corresponding value of Earth (denoted by subscript E), with $R_{\mathrm{E}}=6371 \mathrm{~km}\left(\right.$ Cain et al. 1995) and $M_{\mathrm{E}}=6.0 \cdot 10^{24} \mathrm{~kg}$ (Raith 1997), respectively. An orbital distance of $d=0.2 \mathrm{AU}$ is chosen, so that the planets are located within the habitable zone.

\begin{tabular}{|l||c|c||c|c|c|}
\hline Planet & $R_{\star}\left[R_{\odot}\right]$ & $M_{\star}\left[M_{\odot}\right]$ & $R_{\mathrm{p}}\left[R_{\mathrm{E}}\right]$ & $M_{\mathrm{p}}\left[R_{\mathrm{E}}\right]$ & $d[\mathrm{AU}]$ \\
\hline \hline Earth & $0.46^{a}$ & $0.5^{a}$ & 1.0 & 1.0 & $0.2^{a}$ \\
Mercury & $0.46^{a}$ & $0.5^{a}$ & $0.38^{b}$ & $0.055^{c}$ & $0.2^{a}$ \\
Large Earth & $0.46^{a}$ & $0.5^{a}$ & $1.63^{d}$ & $6.0^{d}$ & $0.2^{a}$ \\
Ocean Planet & $0.46^{a}$ & $0.5^{a}$ & $2.0^{d}$ & $6.0^{d}$ & $0.2^{a}$ \\
\hline
\end{tabular}

Table 2.3: Parameters for different terrestrial planets. All values are given in units normalised to Earth. Sources of the parameters: ${ }^{(a)}$ see text, ${ }^{(b)}$ Cain et al. (1995), ${ }^{(c)}$ Raith (1997), ${ }^{(d)}$ Léger et al. (2004). 


\title{
3 Tidal interaction
}

\author{
As far as we can determine, peanut butter \\ has no effect on the rotation of the earth. \\ George August, Ph.D., et al., JIR 39, 22, 1994
}

Planets in an orbit around a central star are subject to tides. These tides arise because of the finite extension of the planet in the inhomogeneous gravitational field of the central body. In other words, tides are caused by the gravitational gradient across the planet. Similarly, the planet induces tides on the central star, which act back on the planet. Because of the strong dependence of the gravitational gradient on the orbital distance, planets in close-in orbits experience a much stronger tidal interaction than the planets of the solar system. This leads to a large variety of effects, most of which are of no importance for the solar system planets. In this respect, at least the following effects should be mentioned:

- The continuous action of tides is known to reduce the planetary rotation rate. In the solar system, the spin rates of Mercury and Venus are substantially retarded by the Sun (Goldreich and Soter 1966). This effect is also important for all of the closer moons in the solar system (Table 4-3 in Hubbard 1984, Peale 1999, Table 1). It is frequently referred to as "despin", "spin-down", or "tidal locking", because it eventually results in a synchronous rotation (i.e. the time for one rotation and one orbit around the central body become identical). Shortly after the discovery of the first extrasolar planets, the timescale for the despin of close-in planets $(d \lesssim 0.1 \mathrm{AU})$ was studied (Guillot et al. 1996, Marcy et al. 1997), finding that for such planets the timescale for this effect is remarkably short. For example, for a hypothetical Jupiter-like planet orbiting a solar twin at $0.05 \mathrm{AU}$, the synchronisation timescale is approximately $2 \mathrm{Myr}$ (Seager and Hui 2002). This effect is discussed in detail in Section 3.1 .

- Similarly to the evolution of the orbital eccentricity of satellites in the solar system (MacDonald 1964, Goldreich and Soter 1966, Peale|1999), the eccentricity of closein extrasolar planets is strongly affected by tidal interaction. This effect (sometimes called "orbital circularisation" or "eccentricity damping") will be discussed in Section 3.2. The timescale for orbital circularisation is typically much longer than the timescale for synchronous rotation defined above ${ }^{1}$ (Marcy et al. 1997, and Section 3.2).

\footnotetext{
${ }^{1}$ Also note that Mercury is tidally locked in a 3:2 spin-orbit resonance, but has a highly eccentric orbit ( $e=0.206$, see Hubbard 1984).
} 
- The planetary obliquity is defined as the angle between the planetary rotation axis and the vector normal to the orbital plane. In the solar system, the Earth's obliquity (presently $23.45^{\circ}$ ) is increasing at a very small rate because of the tides induced by the Sun ${ }^{2}$ (MacDonald 1964). For close-in planets, however, tidal interaction decreases the obliquity. For planets in circular orbits, the timescale for obliquity damping is identical to the timescale for synchronous rotation defined above (Peale 1999). This effect is described in more detail in Section 3.3 .

- In analogy to the Earth-Moon system, where tidal interaction changes the inclination of the lunar orbit (MacDonald 1964), the inclination of an exoplanetary orbit around its star is influenced by tidal interaction. However, this is a slow process, and the timescale for reaching coplanarity (i.e. the coincidence of the planetary orbital plane with the stellar equatorial plane) is larger than the timescale for synchronous rotation defined above (Seager and Hui|2002, and references therein).

- When tides are moving in the planetary rest frame, the periodic distortion of the planet is accompanied by dissipation of energy (Hubbard 1984, Chapter 4). This produces an additional term in the planetary energy balance. While this tidal heating is negligibly small for the terrestrial planets of the solar system, it is the dominating contribution in the heat balance of Jupiter's close-in moon Io (Hubbard 1984. Chapter 8). At the same time, the dissipation of tidal energy is almost certainly responsible for the active volcanism on Io (Peale 1999). Bodenheimer et al. (2003) examined the influence of tidal heating on the evolution of the radii of close-in extrasolar planets. They found that, for this effect to be effective over long periods of time, an additional planetary companion is required to continuously induce an orbital eccentricity. Not only the energy gained by circularising an eccentric orbit, but also the energy gain connected to the planetary spin-down can strongly heat up a planet. However, this additional energy source is only active until the planet achieves synchronous rotation. For HD 209458b, Showman and Guillot (2002) estimate that the thermal pulse associated with the initial spin-down of the planet could affect the planetary radius for a limited period of time.

- In a similar way to the "tidal locking" of a planet (see above), tidal effects can modify the stellar rotation (Marcy et al. 1997). Through this effect, the stellar rotation is increased in the case of a slowly rotating star (when compared to the planetary orbital frequency). In the case of a rapidly rotating star, the stellar rotation is spun down. Pätzold et al. (2004) showed that the effect of tides on the stellar rotation can be considerable, increasing the stellar rotation rate by a factor of a few in some cases. For a correct treatment of the evolution of the stellar rotation rate, magnetic braking of the star by its magnetised wind has to be taken into account (Dobbs-Dixon et al.|2004).

- The change of the stellar rotation rate also has consequences for the planet: Because of the conservation of angular momentum, a change of the stellar rotation by tidal

${ }^{2}$ This situation is, of course, complicated by the Earth-Moon interaction (MacDonald 1964). Close-in extrasolar planets $(d \lesssim 0.05 \mathrm{AU})$, however, can only have satellites of very low masses $\left(\lesssim 10^{-4}\right.$ Earth masses, i.e. $\lesssim 10^{-2}$ times the mass of the Moon, for a solar-mass star of $4.6 \mathrm{Gyr}$ age, see Barnes and O'Brien 2002), so that this effect is negligible here. 
effects directly implies a change of the planetary orbital angular momentum. In this way, the evolution of the stellar rotation and of the planetary semi-major axis are coupled. Therefore, the planetary semi-major axis decreases, until the planet finally reaches the Roche limit defined as the distance below which the tidal force of the star becomes larger than the self-gravitation of the planet. According to Murray and Dermott (1999), $d_{\text {Roche }}=2.46 R_{\star}$. Pätzold and Rauer (2002) showed that for very small orbital distances $(\lesssim 0.05 \mathrm{AU})$, the Roche limit is reached within a timescale of less than $1 \mathrm{Gyr}$ (see, e.g. Figure 2 of that work). This result does not change appreciably for planets with eccentric orbits (Jiang et al.|2003). Pätzold et al. (2004) studied the importance of this effect for the presently known transiting planet, finding time scales for the spiralling of the planet into the central star of the order of 1 Gyr.

- Finally, tidal interaction is also expected to locally heat the star close to the subplanetary point (Cuntz et al. 2000). Different searches were conducted to detect this effect, but so far no local increase of stellar activity due to tidal interaction could be identified. These searches (along with the search for a signature of magnetic interaction) are briefly described in Section 2.5 .

In this work, the first three points of this list are carefully analysed for close-in exoplanets. At this point it should be noted that a planet may escape the evolution to synchronous rotation, to a circular orbit, or to zero obliquity in the presence of other nearby planets (Laskar and Robutel 1993, Seager and Hui 2002, Atobe et al. 2004). As most exoplanetary host stars have only one known planet, this work is restricted to the case of a single planet in an orbit around a single star is treated.

The aim of this section is threefold: Section 3.1 discusses which close-in extrasolar planets can be expected to be tidally locked. This is important with respect to the strength of the planetary magnetic moment. Section 3.2 studies whether the planets are likely to be in circular orbits, which is relevant, for example, to determine the stellar wind characteristics at the planetary location. Section 3.3 analyses the orientation of the planetary rotation axis, which relates to the orientation of the planetary magnetic moment.

\subsection{Tidal locking}

In this Section, the despinning of the planetary rotation through tidal interaction is discussed. Figure 3.1 schematically illustrates this effect for a planet on a close-in circular orbit: First, the tidal force of the star (i.e. the gradient of the stellar gravitation across the planetary diameter) creates two tidal bulges on the planet. For a rapidly rotating planet (i.e. with short rotation period relative to the orbital period), the tidal bulges are displaced from the line connecting the stellar and planetary centre. Hence, the stellar gravitation exerts a torque upon the tidal bulges. Because the tidal bulges are coupled to the planet by dissipative processes, this reduces the planetary rotation rate. After a certain time, an equilibrium state is reached, in which the planetary rotation period equals the time for one full orbit around the star. Then, it is always the same side of the planet which is facing the star (similar to the Earth-Moon system). In this case, the tidal bulge is exactly on the line connecting star and planet, and tidal interaction ceases to influence the planetary rotation. 


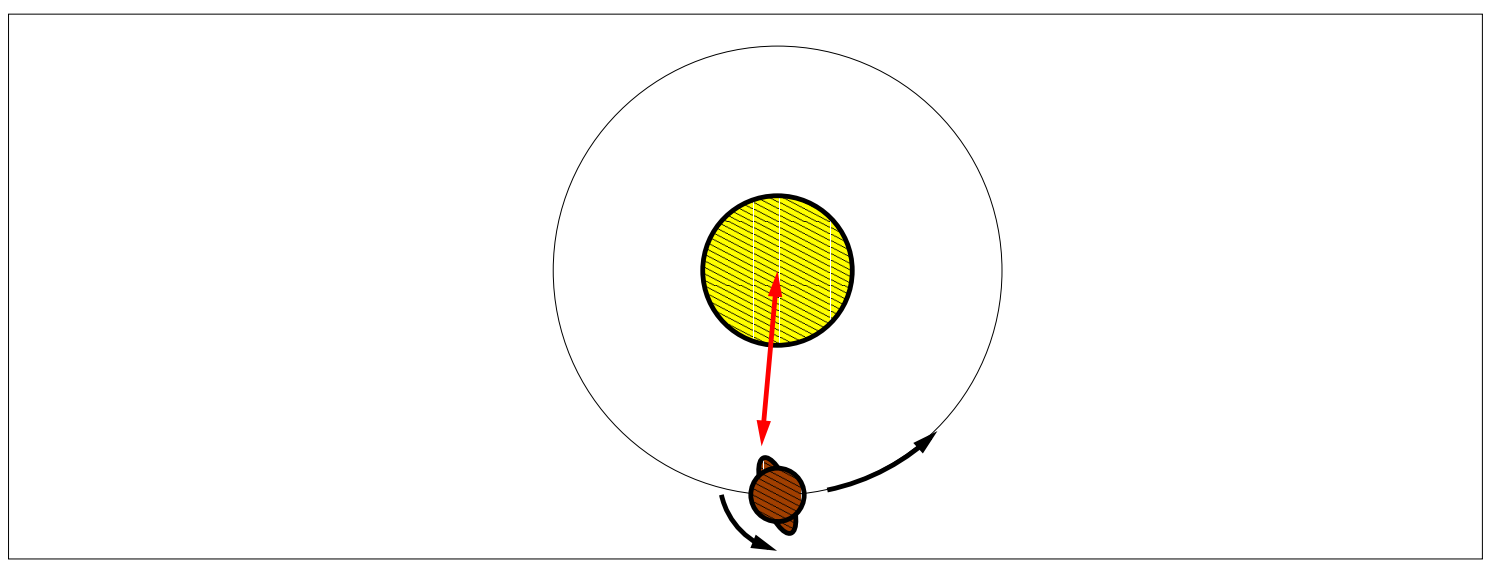

Figure 3.1: Tidal interaction (schematic view). See text for description.

The equations required to calculate the timescale for this process are given in Section 3.1.1. These are then applied to extrasolar gas giants (Sections 3.1.3 and 3.1.4) and to terrestrial exoplanets (Sections 3.1.5 and 3.1.6.

\subsubsection{Tidal locking timescale}

Because of tidal interaction, the planetary rotation gradually slows down from its initial value $\omega_{\mathrm{i}}$ until it reaches the final value $\omega_{\mathrm{f}}$ after tidal locking is completed. In the following, the tidal locking timescale for reaching $\omega_{\mathrm{f}}$ is calculated under the following simplifying assumptions: prograde orbit, spin parallel to orbit (i.e. zero obliquity), and zero eccentricity (Murray and Dermott 1999, Chapter 4). In first order approximation, the frequency $\omega_{\mathrm{f}}$ is equal to the orbital frequency $\omega_{\text {orbit }}$, which is simply given by Kepler's third law:

$$
\omega_{\text {orbit }}=\sqrt{\frac{M_{\star} G}{d^{3}}}
$$

where $M_{\star}$ denotes the mass of the central star, $G$ is the constant of gravitation, and $d$ is the semi-major axis of the planetary orbit.

The rate of change of the planetary rotation velocity $\omega$ for a planet with a mass of $M_{\mathrm{p}}$ and radius of $R_{\mathrm{p}}$ is given by (Goldreich and Soter 1966, Murray and Dermott 1999):

$$
\frac{d \omega}{d t}=\frac{9}{4} \frac{1}{\alpha Q_{\mathrm{p}}^{\prime}}\left(\frac{G M_{\mathrm{p}}}{R_{\mathrm{p}}^{3}}\right)\left(\frac{M_{\star}}{M_{\mathrm{p}}}\right)^{2}\left(\frac{R_{\mathrm{p}}}{d}\right)^{6},
$$

where the constant $\alpha$ depends on the internal mass distribution within the planet ${ }^{3}$. It is defined by $\alpha=I /\left(M_{\mathrm{p}} R_{\mathrm{p}}^{2}\right)$, where $I$ is the planetary moment of inertia. For a sphere of homogeneous density, $\alpha$ is equal to $2 / 5$, so that for planets $\alpha \leq 2 / 5$. $Q_{\mathrm{p}}^{\prime}$ is the modified $Q$-value of the planet. It is defined by

$$
Q_{\mathrm{p}}^{\prime}=Q_{\mathrm{p}}\left(1+\frac{19 \mu}{2 g \rho R_{\mathrm{p}}}\right)
$$

\footnotetext{
${ }^{3}$ Note that in many publications "factors of order unity" are omitted, i.e. $\frac{4}{9} \alpha$ is neglected.
} 
where $\mu$ denotes the planetary rigidity, $g$ is the planetary surface gravity, and $\rho$ is the density within the tidal bulge (which can approximately be set equal to the mean density of the planet, Goldreich and Soter 1966). $Q_{\mathrm{p}}$ is the planetary tidal dissipation factor (the larger it is, the smaller is the tidal dissipation), defined by (MacDonald 1964, Goldreich and Soter 1966):

$$
Q_{\mathrm{p}}^{-1}=\frac{1}{2 \pi E_{0}} \oint \frac{d E}{d t} d t
$$

Here, $E_{0}$ is the maximum energy stored in the tidal distortion, and the integral over $d E / d t$, the energy dissipation rate, is the energy lost during one complete cycle. For large planets, in which self-gravitation far exceeds the rigidity, the contribution of the latter to the restoring force against tidal deformation can be neglected, and one finds $Q_{\mathrm{p}}^{\prime} \approx Q_{\mathrm{p}}$ (Goldreich and Soter 1966). For much smaller bodies, the correction due to rigidity can reach very high values (up to a factor of $10^{7}$ for the Martian satellite Phobos, see Hubbard 1984, Chapter 4). An alternative notation is given by (Murray and Dermott 1999)

$$
Q_{\mathrm{p}}^{\prime}=\frac{3 Q_{\mathrm{p}}}{2 k_{2, \mathrm{p}}}
$$

where $k_{2, \mathrm{p}}$ is the Love number of the planet.

The time scale for tidal locking is obtained by a comparison of the planetary angular velocity and its rate of change:

$$
\tau_{\text {sync }}:=\frac{\omega_{\mathrm{i}}-\omega_{\mathrm{f}}}{\dot{\omega}}
$$

A planet with angular velocity $\omega_{\mathrm{i}}$ at $t=0$ (i.e. after formation) will gradually lose angular momentum, until the angular velocity reaches $\omega_{\mathrm{f}}$ at $t=\tau_{\text {sync }}$. Insertion of eq. (3.2) into eq. (3.6) yields the following expression for $\tau_{\text {sync }}$ :

$$
\tau_{\text {sync }} \approx \frac{4}{9} \alpha Q_{\mathrm{p}}^{\prime}\left(\frac{R_{\mathrm{p}}^{3}}{G M_{\mathrm{p}}}\right)\left(\omega_{\mathrm{i}}-\omega_{\mathrm{f}}\right)\left(\frac{M_{\mathrm{p}}}{M_{\star}}\right)^{2}\left(\frac{d}{R_{\mathrm{p}}}\right)^{6} .
$$

The different dependencies in equation (3.7) are:

- Because of the more efficient dissipation of energy (i.e. lower value of $Q_{\mathrm{p}}^{\prime}$ ), tidal locking occurs faster for a terrestrial planet than for a gaseous giant.

- A larger value of $\omega_{\mathrm{i}}$ (or smaller value of $\omega_{\mathrm{f}}$ ) indicates that more time is required to lose the corresponding angular momentum.

- The larger the mass of the star, the stronger is the tidal bulge it can induce in the planet. Also, the gravitational pull on the tidal bulge depends on the stellar mass. A large planetary mass, on the other hand, reduces the height of the tidal bulge and thus diminishes the tidal interaction.

- The importance of this effect strongly depends on the distance $\left(\tau_{\text {sync }} \propto d^{6}\right)$. Thus, a planet in a close-in orbit ( $d \lesssim 0.1 \mathrm{AU})$ around its central star is subject to strong tidal interaction, leading to gravitational locking on a very short timescale. 
Finally, it should be noted that for planets in eccentric orbits, tidal interaction does not lead to the synchronisation of the planetary rotation with the orbital period. Instead, the rotation period also depends on the orbital eccentricity. At the same time, the timescale to reach this equilibrium rotation rate is reduced (Laskar and Correia 2004). In this work, however, the focus lies on planets in circular orbits, see Section 3.2.

\subsubsection{Imperfect tidal locking}

To be exact, the planets should not be expected to be precisely tidally locked. The thermal atmospheric tides resulting from stellar heating can drive planets away from synchronous rotation (Showman and Guillot 2002, Correia et al. 2003, Laskar and Correia|2004). This mechanism works as follows (Correia and Laskar 2003a): The planetary atmosphere is heated above the substellar point, leading to pressure gradients. A new equilibrium can only be reached by a redistribution of the atmospheric mass (i.e. by a density increase in the low-temperature regions). The pressure redistribution can be represented as a superposition of a (weak) diurnal tide and a stronger semidiurnal tide (Correia and Laskar 2003a, Figure 3). The gravitational torque on the atmosphere changes the atmospheric rotation, which, depending on the coupling between the planetary interior and its atmosphere, may or may not influence the planetary rotation rate 4 . Such thermal atmospheric tides are also the cause for the slight retrograde rotation of the planet Venus in the solar system (MacDonald 1964, Correia and Laskar 2001, Correia et al. 2003, Correia and Laskar 2003a), where the present state is characterised by an equilibrium between gravitational and thermal atmospheric tidal torques (Correia et al. 2003).

Showman and Guillot (2002) estimated the deviation from synchronous rotation resulting from atmospheric tides for a typical Hot Jupiter. As an upper limit, it was found that the error introduced for $\omega_{\mathrm{f}}$ by this effect could be of the same order of magnitude as the value given by eq. (3.1). For this reason, $\omega_{\mathrm{f}}$ should be considered to be exact only up to a factor of two.

A similar situation holds for terrestrial planets with dense atmospheres. The example of Venus shows that for such a case, the effect is clearly important. Correia and Laskar (2001) and Correia et al. (2003) found that in the presence of planetary perturbations synchronous rotation is not stable for a planet with a dense atmosphere, like Venus. On the other hand, if Earth-like atmospheres are considered, the deviation should be much smaller, and for terrestrial exoplanets without an atmosphere it vanishes. Because no better estimation exists, the maximum (tidally locked) rotation rate is assumed to be twice the value given by eq. (3.1).

In this work, the importance of such asynchronous rotation is studied by comparing results obtained using $\omega_{\mathrm{f}}=\omega_{\text {orbit }}$, with $\omega_{\text {orbit }}$ from eq. (3.1), and results found when using twice that value (i.e. $\omega_{\mathrm{f}}=2 \omega_{\text {orbit }}$ ). For the tidal locking timescale, the opposite limit (where thermal atmospheric tides slow down the planetary rotation instead of increasing it) will also be considered, i.e. the rotation rate $\omega_{\mathrm{f}}$ of a tidally locked planet is assumed to lie in the range $0 \leq \omega_{\mathrm{f}} \leq 2 \omega_{\text {orbit }}$ with $\omega_{\text {orbit }}$ given by eq. (3.1). The influence this

\footnotetext{
${ }^{4}$ The picture is further complicated by the fact that the nonuniform distribution of atmospheric mass deforms the planet and creates a "pressure bulge", which in turn gravitationally interacts with the star and with the atmospheric mass distribution. Also, the atmospheric mass interacts with the tidal bulge of the planet (Correia and Laskar|2003a).
} 
has on the timescale for tidal locking $\tau_{\text {sync }}$ will be discussed in Section 3.1 .3 for gaseous giant exoplanets, and in Section 3.1.5 for terrestrial exoplanets. Similarly, the influence of imperfect tidal locking on the expected magnetic moment of such planets is studied in Section 4.4 and Section 4.6 .

\subsubsection{Parameters for gas giants}

In this section, the parameters $\left(\alpha, Q_{\mathrm{p}}^{\prime}, \omega_{\mathrm{i}}\right.$ and $\left.\omega_{\mathrm{f}}\right)$ required to calculate the timescale for tidal locking of Hot Jupiters (as defined in Section 2.2.4) are discussed. The calculation itself will be presented in Section 3.1.4. The parameters used are listed in Table 3.1

\subsubsection{Structure parameter $\alpha$}

For large gaseous planets, the equation of state can be approximated by a polytrope of index $\kappa=1$ (see Section 4.3). In that case, the structure parameter $\alpha$ (defined by $\alpha=$ $\left.I / M_{\mathrm{p}} R_{\mathrm{p}}^{2}\right)$ is found to be given by $\alpha=0.26$ (Gu et al. 2003$)$.

\subsubsection{Tidal dissipation factor $Q_{\mathrm{p}}^{\prime}$}

As noted before, for planets with masses of the order of one Jupiter mass, one finds $Q_{\mathrm{p}}^{\prime} \approx Q_{\mathrm{p}}\left(\right.$ Goldreich and Soter 1966). More precisely, $k_{2, \mathrm{p}}$ has a value of $k_{2, \mathrm{p}} \approx 0.5$ for Jupiter (Murray and Dermott 1999, Laskar and Correia 2004) and $k_{2, \mathrm{p}} \approx 0.3$ for Saturn (Peale 1999, Laskar and Correia 2004). Because the focus lies on Jupiter-like planets, a value of $k_{2, \mathrm{p}}=0.5$ will be used in this work. With eq. (3.5), this results in $Q_{\mathrm{p}}^{\prime} \approx 3 Q_{\mathrm{p}}$.

In principle, the value of $Q_{\mathrm{p}}$ is not only a function of the mass, structure and composition of the respective planet, but it can also depend both on the frequency and the amplitude of the tidal forcing (MacDonald 1964, Ogilvie and Lin 2004). While for solid bodies the frequency dependency of $Q_{\mathrm{p}}$ is usually negligible (see Section 3.1.5.2 for discussion), for gaseous giant planets, a correct treatment should include the atmospheric response to the tidal potential (Peale 1999). In fact, the response of the planet to tidal forcing should be separated into a frequency-independent "equilibrium tide" (i.e. the reaction of a homogeneous and initially spherical body constantly adjusting its shape to maintain quasi-hydrostatic equilibrium) and the additional, frequency dependent contribution of the "dynamical tide" (Ogilvie and Lin 2004). The dynamical tide constitutes a correction due to the (resonant) excitation of waves (Lubow et al. 1997, Ogilvie and Lin 2004). In the following, only the contribution of the "equilibrium tide" is considered. It is the conventional procedure to use this simplification (Hubbard 1984, Gu et al. 2003, Dobbs-Dixon et al. 2004), which is necessary for a fast calculation of the spindown timescale $\tau_{\text {sync }}$.

For Jupiter, the lower limit for the value of the tidal dissipation factor $Q_{\mathrm{p}}$ is much better understood than the upper limit. The lower limit was obtained from the observed orbits of the inner satellites. Through the action of tides, the orbits of the satellites expand. From the current orbital distance and the assumed age of the planet-satellite system, an upper limit can be placed on the strength of the planet-satellite interaction, leading to the minimum allowed value, $Q_{\mathrm{p} \text {,min }}$ (corresponding to maximum tidal interaction). For values smaller than this $Q_{\mathrm{p}, \mathrm{min}}$, the satellites would have been located at the planetary surface 4.6 
Gyr ago (Goldreich and Soter 1966, Hubbard 1984, Peale 1999). The upper limit, $Q_{\mathrm{p}, \max }$, can be determined, for example, from the eccentricity of Io, which is nearly the equilibrium value (Peale 1999). For Jupiter, this leads to the following range of allowed values: $6.6 \cdot 10^{4} \lesssim Q_{\mathrm{p}} \lesssim 2.0 \cdot 10^{6}$ (Peale 1999). Several estimations of the turbulent dissipation within Jupiter yield $Q_{\mathrm{p}}$-values larger than this upper limit, while other theories predict values consistent with this upper limit (Marcy et al. 1997, Peale 1999, and references therein). This demonstrates that the origin of the value of $Q_{\mathrm{p}}$ is not well understood even for Jupiter (Marcy et al. 1997).

Extrasolar giant planets are subject to strongly different conditions, and it is difficult to constrain $Q_{\mathrm{p}}$. Typically, Hot Jupiters are assumed to behave similarly to Jupiter, and values in the range of $Q_{\mathrm{p}}^{\prime} \approx 1.0 \cdot 10^{5} \ldots 1.0 \cdot 10^{6}$ are used ${ }^{5}$ (Guillot et al. 1996, Seager and Hui 2002, Showman and Guillot 2002, Gu et al. 2003, Dobbs-Dixon et al. 2004).

It should be noted that the value of $Q_{\mathrm{p}}$ is not necessarily constant with time, as the size of the convective region within Jupiter may have changed during gradual cooling of the planet (Peale 1999). Similarly, the size of the convective region may be different for strongly insulated Hot Jupiters. For a synchronised Hot Jupiter, Ogilvie and Lin (2004) find that $Q_{\mathrm{p}}$ is larger by a factor of 50 when compared to Jupiter (namely $\approx 50 \cdot 10^{5}$ ). This results from the slow planetary rotation. In this section, however, the aim is to find the time necessary to reach this state, so that this additional increase is not required. For the effect of orbital circularisation (discussed in Section 3.2), the increased value of $Q_{\mathrm{p}}$ could increase the timescale $\tau_{\text {circ }} \propto Q_{\mathrm{p}}^{\prime}$ by the same factor.

In view of the uncertainties involved, in this work the value of $Q_{\mathrm{p}}^{\prime}$ will be taken to be $10^{6}$ when determining the area in parameter space where tidal locking occurs quickly $\left(\tau_{\text {sync }} \leq 100 \mathrm{Myr}\right)$, whereas $Q_{\mathrm{p}}^{\prime}=10^{5}$ will be used when determining the region where the tidal locking timescale is long ( $\tau_{\text {sync }} \geq 10 \mathrm{Gyr}$ ). Thus, the area of "potentially locked" planets (see below) is increased. As far as the orbital circularisation is concerned, this procedure yields a value of $Q_{\mathrm{p}}$ close to that of Ogilvie and Lin (2004) for the slowly rotating regime.

\subsubsection{Initial rotation rate $\omega_{\mathrm{i}}$}

Unfortunately, $\omega_{\mathrm{i}}$ is not well constrained by planetary formation theories. The relation between the planetary angular momentum density and planetary mass observed in the solar system (MacDonald 1964) suggests a primordial rotation period of the order of 10 hours (Hubbard 1984, Chapter 4). In the following, the initial rotation rate of a gaseous giant planet is assumed to be equal to the current rotation rate of Jupiter, i.e. $\omega_{i}=\omega_{\mathrm{J}}$ with $\omega_{\mathrm{J}}=1.77 \cdot 10^{-4} \mathrm{~s}^{-1}$ (Raith 1997).

\subsubsection{Final rotation rate $\omega_{\mathrm{f}}$}

For the systems discussed here, the synchronous rotation rate $\omega_{\text {orbit }}$ given by Kepler's law, eq. (3.1), is of the same order of magnitude as the initial rotation rate $\omega_{\mathrm{i}}$. The maximum

\footnotetext{
${ }^{5}$ In some of these publications, the notation is different, and the symbol $Q_{\mathrm{p}}$ is used instead of $Q_{\mathrm{p}}^{\prime}$.
} 
value of $\omega_{\text {orbit }}$ used is estimated in the following way:

$$
\omega_{\text {orbit }}^{\max }=\sqrt{\frac{M_{\star}^{\max } G}{d_{\text {min }}^{3}}} .
$$

For the giant planets discussed in this work, the extreme values are $M_{\star}^{\max }=1.5 M_{\odot}$ and $d_{\min }=0.02 \mathrm{AU}$, so that $\omega_{\text {orbit }}$ is limited by

$$
\omega_{\text {orbit }} \leq \omega_{\text {orbit }}^{\max }=8.6 \cdot 10^{-5} \mathrm{~s}^{-1},
$$

corresponding to a period of 20.2 hours. If one compares this to the typical initial rotation rates of 10 hours, it is not clear whether the contribution of $\omega_{\mathrm{f}}$ can be neglected in eq. (3.7). For this reason, it will be included. According to Section 3.1.2, the value of $\omega_{\mathrm{f}}$ lies in the range $0 \leq \omega_{\mathrm{f}} \leq 2 \omega_{\text {orbit }}$, where $\omega_{\text {orbit }}$ is given by eq. (3.1). Here, $\omega_{\mathrm{f}}=2 \omega_{\text {orbit }}$ is used when calculating the outer boundary of the "potentially tidally locked" region (see below), and $\omega_{\mathrm{f}}=0$ is used for the inner boundary of this region. This procedure increases the size of the "potentially tidally locked" region.

\subsubsection{Overview of the parameters}

All parameters used for the calculation of the tidal locking time scale for Hot Jupiters can be found in Table 3.1. Because several of the parameters are not known precisely, two different sets of parameters are used to find the upper and lower boundary of the region in parameter space for which tidal locking is possible, but not certain, see Section 3.1.4. The planetary mass $M_{\mathrm{p}}$ and radius $R_{\mathrm{p}}$ are given in units normalised to Jupiter (denoted by subscript J), with $R_{\mathrm{J}}=71492 \mathrm{~km}$ (Cain et al. 1995) and $M_{\mathrm{J}}=1.9 \cdot 10^{27} \mathrm{~kg}$ (Raith 1997).

\begin{tabular}{|l||c|c|c|c|c|c|}
\hline Planet & $\alpha$ & $Q_{\mathrm{p}}^{\prime}$ & $R_{\mathrm{p}}\left[R_{\mathrm{J}}\right]$ & $M_{\mathrm{p}}\left[M_{\mathrm{J}}\right]$ & $\omega_{\mathrm{i}}\left[\omega_{\mathrm{J}}\right]$ & $\omega_{\mathrm{f}}$ \\
\hline \hline Jupiter & $0.26^{a}$ & $10^{5} \ldots 10^{6}$ & 1.0 & 1.0 & 1.0 & $0 \ldots 2 \omega_{\text {orbit }}$ \\
HD 209458b & $0.26^{a}$ & $10^{5} \ldots 10^{6}$ & $1.42^{b}$ & $0.69^{b}$ & 1.0 & $0 \ldots 2 \omega_{\text {orbit }}$ \\
$\tau$ Bootes (heavy) & $0.26^{a}$ & $10^{5} \ldots 10^{6}$ & $1.48^{c}$ & $10.0^{c}$ & 1.0 & $0 \ldots 2 \omega_{\text {orbit }}$ \\
\hline
\end{tabular}

Table 3.1: Parameters for the calculation of the tidal locking timescale for Hot Jupiters. Planetary masses and radii are given in units normalised to Jupiter. Sources of the parameters: ${ }^{(a)}$ Gu et al. (2003), ${ }^{(b)}$ Cody and Sasselov (2002), ${ }^{(c)}$ see Section 2.6.2.

\subsubsection{Results for gas giants}

In this section, eq. (3.7) is used to calculate the timescale for tidal locking for Hot Jupiters (as defined in Section 2.2.4). It will be shown that most of the observed Hot Jupiters can safely be assumed to be tidally locked. To classify the planets, three different categories are defined:

- Tidally locked planets: These are the planets for which the tidal locking timescale is lower or equal to $100 \mathrm{Myr}\left(\tau_{\text {sync }} \leq 100 \mathrm{Myr}\right)$. 


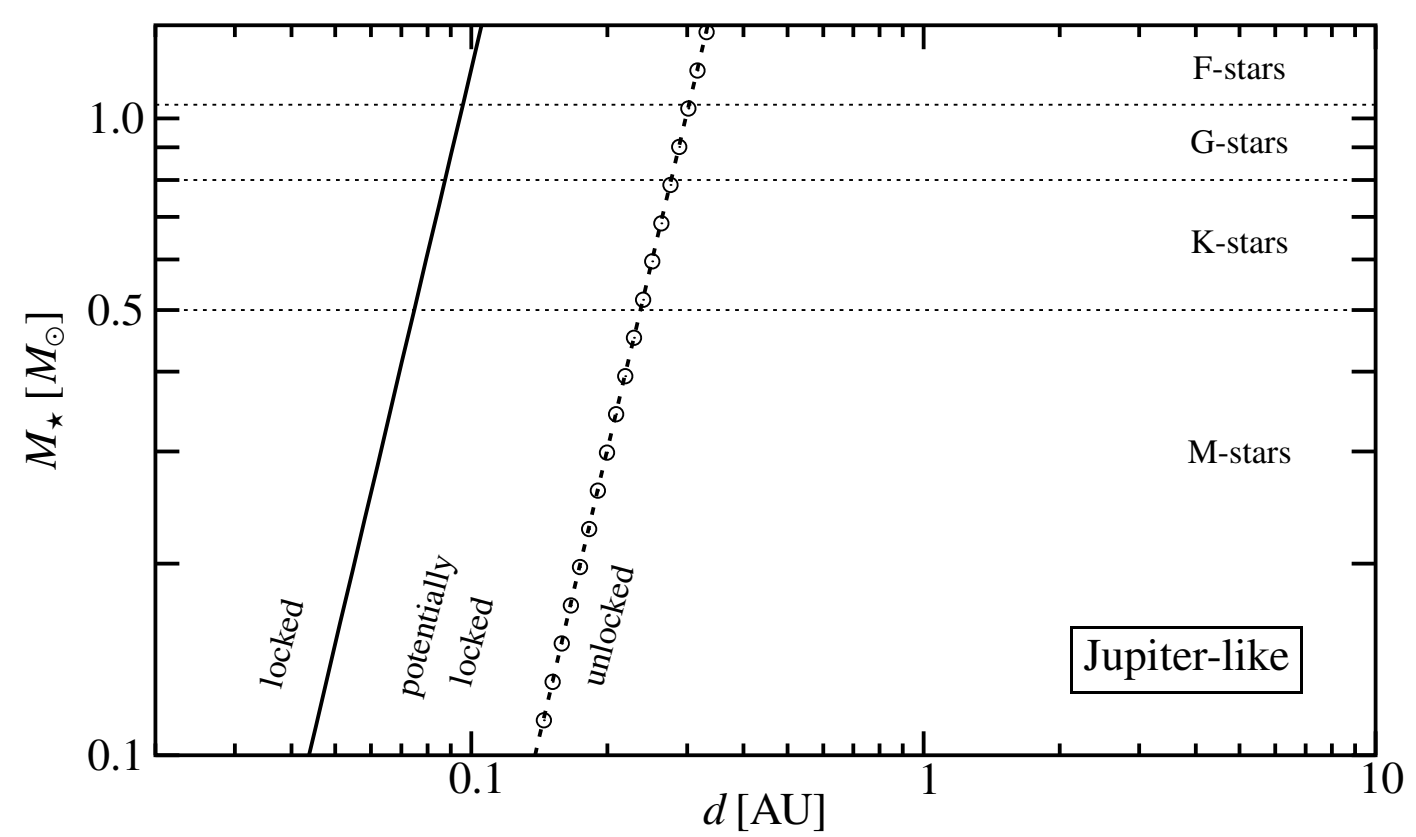

Figure 3.2: Tidal locking regimes for a Hot Jupiter as a function of orbital radius $d$ and mass $M_{\star}$ (or spectral type) of the host star. Solid line: boundary between tidally locked and potentially tidally locked planets. Dashed line, circles: boundary between potentially tidally locked and tidally unlocked planets, neglecting (dashed line) and including (circles) a nonzero value of $\omega_{\mathrm{f}}$.

- Potentially tidally locked planets: For these planets, the tidal locking timescale lies between $100 \mathrm{Myr}$ and $10 \mathrm{Gyr}\left(100 \mathrm{Myr} \leq \tau_{\text {sync }} \leq 10 \mathrm{Gyr}\right)$.

- Unlocked planets: This class contains all planets with $\tau_{\text {sync }} \geq 10 \mathrm{Gyr}$.

The upper and lower boundaries of the potentially locked region are determined by the conditions $\tau_{\text {sync }}=100 \mathrm{Myr}$ and $\tau_{\text {sync }}=10 \mathrm{Gyr}$, respectively. To take into account additional uncertainties, the lower boundary is calculated with $Q_{\mathrm{p}}^{\prime}=10^{6}$ and $\omega_{\mathrm{f}}=0$, whereas the upper boundary is calculated with $Q_{\mathrm{p}}^{\prime}=10^{5}$ and $\omega_{\mathrm{f}}=2 \omega_{\text {orbit }}$, see Table 3.1. This increases the area of the "potentially locked" region.

To visualise these different classes of planets, the planetary parameters $Q_{\mathrm{p}}, R_{\mathrm{p}}, M_{\mathrm{p}}$ and $\omega_{\mathrm{i}}$ are held constant (i.e. the planet is kept unchanged), while the semi-major axis $d$ and the stellar mass $M_{\star}$ are varied. In Fig. 3.2, the different regimes can be seen: The left part of the diagram (from the left border of the diagram to the solid line) contains the points in the $d-M_{\star}$-parameter space where tidal locking occurs very quickly $\left(\tau_{\text {sync }} \leq 0.1 \mathrm{Gyr}\right)$. All planets discovered at such orbital distances will be synchronously rotating. For all planets to the right of the dashed line, the synchronisation timescale is very long ( $\tau_{\text {sync }} \geq 10$ Gyr). Any planet observed in this regime will not have lost much of its initial angular momentum. The region between the solid and the dashed line corresponds to systems in which an observed planet may or may not be tidally locked ( $\left.0.1 \mathrm{Gyr} \leq \tau_{\text {sync }} \leq 10 \mathrm{Gyr}\right)$.

From Fig. 3.2, it also becomes clear that the influence of $\omega_{\mathrm{f}}$ on eq. (3.7) is negligible as far as the upper boundary of the potentially locked regime is concerned (i.e. the circles lie on the dashed line). The reason is that this boundary is located at distances $d_{\min } \geq 0.1$ 


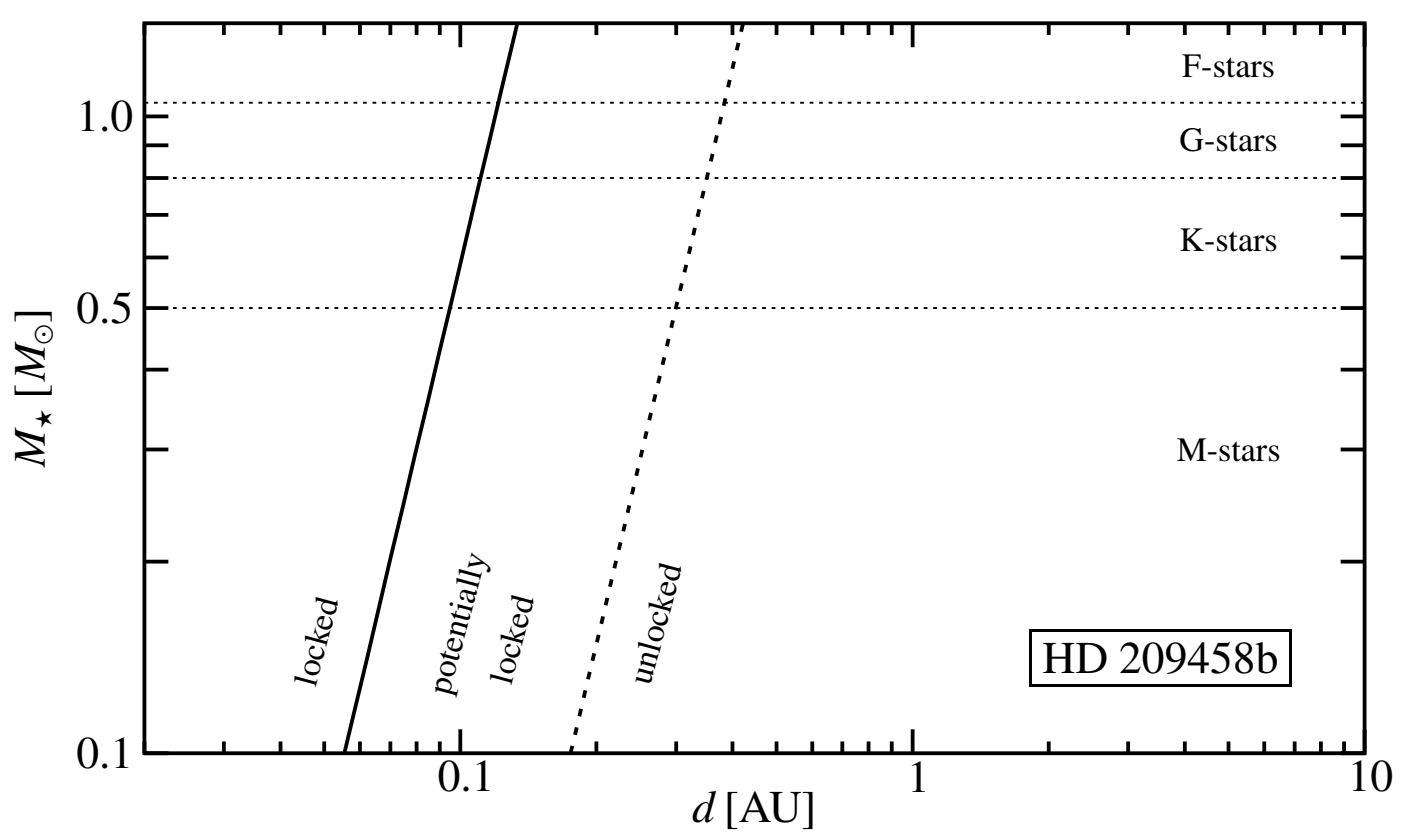

Figure 3.3: Tidal locking regimes for a planet similar to HD 209458b as a function of orbital radius $d$ and mass $M_{\star}$ (or spectral type) of the host star.

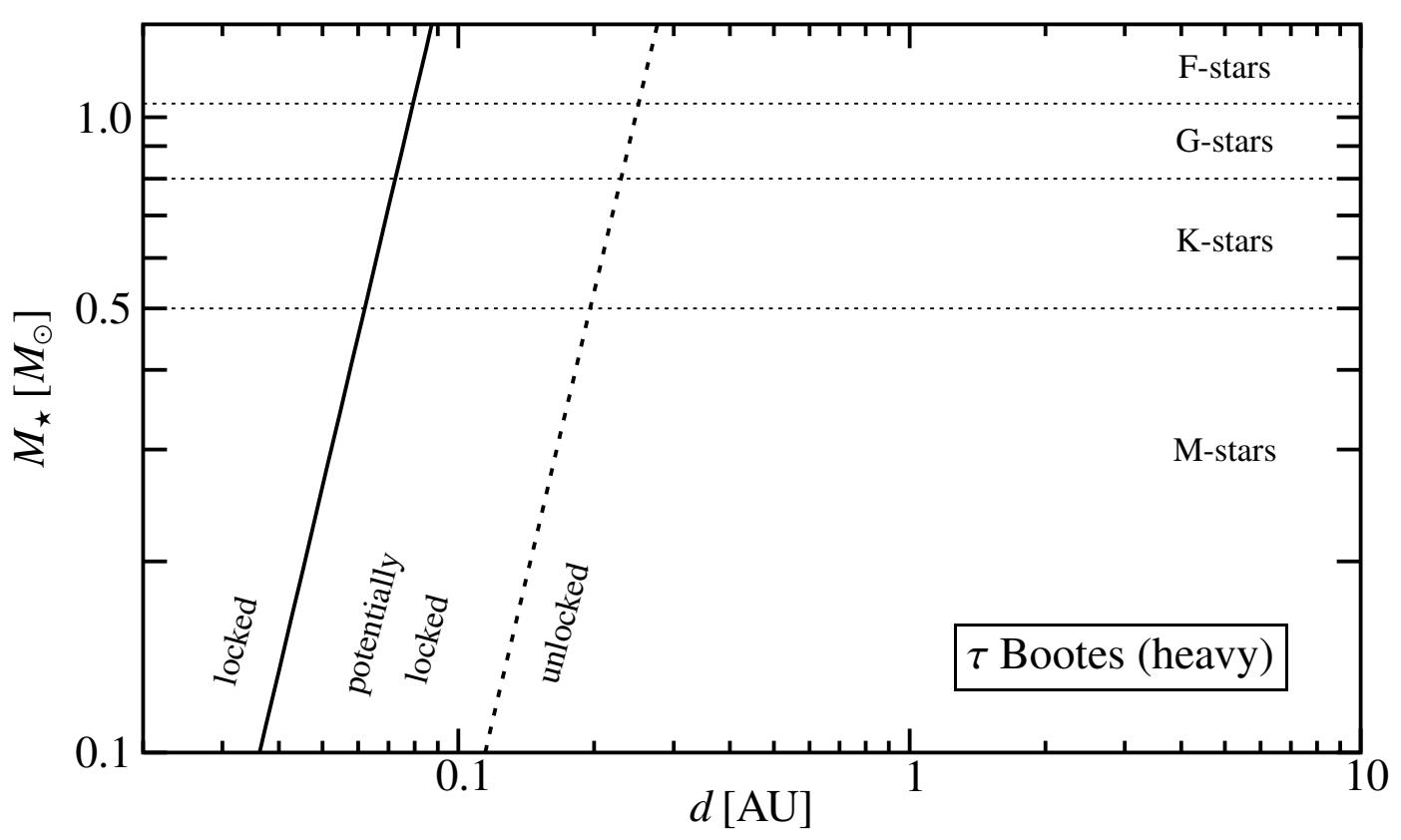

Figure 3.4: Tidal locking regimes for a planet similar to $\tau$ Bootes (heavy model) as a function of orbital radius $d$ and mass $M_{\star}$ (or spectral type) of the host star.

AU, which is much larger than the value used in the simple estimation of eq. (3.9). In the following, $\omega_{\mathrm{f}}$ will be set to zero for gaseous giant planets. 
As can be seen from eq. (3.7), the tidal locking timescale $\tau_{\text {sync }}$ depends on the planetary parameters as $\tau_{\text {sync }} \propto M_{\mathrm{p}} / R_{\mathrm{p}}^{3}$. To demonstrate this influence of the planetary parameters, Figs. 3.3 and 3.4 show the same classification in parameter space as Fig. 3.2, but apply to planets with masses and radii different from Jupiter's values. All other values were kept identical. Fig. 3.3 shows the case of the planet HD 209458b, which has the smallest value $M_{\mathrm{p}} / R_{\mathrm{p}}^{3}$ of all Hot Jupiters examined in this work $\left(M_{\mathrm{p}}=0.69 M_{\mathrm{J}}\right.$, and $\left.R_{\mathrm{p}}=1.42 R_{\mathrm{J}}\right)$. In Fig. 3.4, the opposite limit is examined, which is the case of $\tau$ Bootes $\left(M_{\mathrm{p}}=10.0 M_{\mathrm{J}}\right.$, and $R_{\mathrm{p}}=1.48 R_{\mathrm{J}}$ in the "heavy" model of Section 2.6.2). The comparison shows that, although the differences are not large, the planet HD 209458b with the lower average density is more prone to tidal locking than Jupiter, while for the heavy planet $\tau$ Bootes, the size of the "tidally locked" regime is considerably smaller.

Figures 3.3 and 3.4 show clearly that all Hot Jupiters with orbital radii of $\lesssim 0.08$ AU around solar-mass stars will be tidally locked. All Hot Jupiters treated in this work fall into this distance range.

In the following, the calculation of the tidal locking timescale will be expanded to terrestrial planets.

\subsubsection{Parameters for terrestrial planets}

In this section, the parameters $\left(\alpha, Q_{\mathrm{p}}^{\prime}, \omega_{\mathrm{i}}\right.$ and $\left.\omega_{\mathrm{f}}\right)$ required for the calculation of the tidal locking timescale of terrestrial exoplanets (as defined in Section 2.2.5) are discussed. The calculation itself will then be presented in Section 3.1.6. The different types of terrestrial planets discussed here are the same as in Section 2.6.4. The parameters are summarised in Table 3.2

\subsubsection{Structure parameter $\alpha$}

For the Earth, the structure parameter $\alpha$ is given by $\alpha=1 / 3$ (Goldreich and Soter 1966). In the following, this value will be used for all terrestrial exoplanets.

\subsubsection{Tidal dissipation factor $Q_{\mathrm{p}}^{\prime}$}

As discussed in Section 3.1.1 to have a reasonable estimation for the value of $Q_{\mathrm{p}}^{\prime}$, both $Q_{\mathrm{p}}$ and the Love number $k_{2, \mathrm{p}}$ have to be known. For solid bodies, $Q_{\mathrm{p}}$ is roughly independent of the frequency (MacDonald 1964, Goldreich and Soter 1966, Peale 1999), although at very low frequencies (i.e. tides acting upon a very slowly rotating body) the assumption of constant $Q_{\mathrm{p}}$ should be replaced, for example, by a constant time lag (Correia et al. 2003). During despin, however, the approximation of a frequency independent "equilibrium tide" value of $Q_{\mathrm{p}}$ seems reasonable, because the time spent in the slowly rotating regime is much smaller than the total time to reach the final state.

For the tidal dissipation factor $Q_{\mathrm{p}}$ and the Love numbers $k_{2, \mathrm{p}}$, the values given by Murray and Dermott (1999) are adopted. According to Murray and Dermott (1999), $k_{2, \mathrm{p}} \approx 0.1$ for Mercury, and $k_{2, \mathrm{p}}=0.3$ for Earth (MacDonald 1964, Murray and Dermott|1999). The latter value will also be used for the "Large Earth" and the "Ocean Planet" case.

For the Earth, a value of $Q_{\mathrm{p}} \approx 12$ can be determined from the measured secular acceleration of the moon (Goldreich and Soter 1966, Murray and Dermott 1999). This 
value is relatively small when compared to other terrestrial planets, where $Q_{\mathrm{p}}$ is typically of the order of $10^{2}$. This is probably due to the fact that, for the Earth, much energy is dissipated in the shallow seas (MacDonald 1964, Hubbard 1984, Kasting et al. 1993, Murray and Dermott 1999). In the past, when the continents were joined, the value of $Q_{\mathrm{p}}$ was probably larger (Peale 1999, Murray and Dermott 1999). For this reason, and also because an Earth-like planet without shallow seas is equally interesting to study, a second case called "Ancient Earth" is included in the comparison. For this case, $Q_{\mathrm{p}}$ is set to the value usually assumed for Venus and Mercury $\left(Q_{\mathrm{p}} \approx 100\right.$, Murray and Dermott 1999), as it was also done by Kasting et al. (1993). The same value is assumed for the case of the "Large Earth" and the "Ocean Planet".

From $k_{2, \mathrm{p}}$ and $Q_{\mathrm{p}}$, the required value of $Q_{\mathrm{p}}^{\prime}$ can be obtained using eq. 3.5 . The resulting values can be found in Table 3.2 .

\subsubsection{Initial rotation rate $\omega_{\mathrm{i}}$}

The initial rotation rate $\omega_{\mathrm{i}}$ of a terrestrial planet is a poorly known quantity (see, e.g. Correia and Laskar 2003a). It certainly depends on the details of the planetary formation and can be strongly influenced by processes like migration or impacts. Therefore, two limits for $\omega_{\mathrm{i}}$ are considered:

- a relatively high initial rotation rate as suggested for the early Earth-Moon system, with $\omega_{\mathrm{i}}=1.83 \omega_{\mathrm{E}}$ corresponding to a length of day of 13.1 h (MacDonald 1964)

- a lower rotation rate with $\omega_{\mathrm{i}}=0.80 \omega_{\mathrm{E}}$ corresponding to a day of $30 \mathrm{~h}$.

For $\omega_{\mathrm{E}}$, the value of $\omega_{\mathrm{E}}=7.27 \cdot 10^{-5} \mathrm{~s}^{-1}$ is used. Note that a primordial rotation period of the order of 10 hours is consistent with the relation between the planetary angular momentum density and planetary mass observed in the solar system (Hubbard 1984, Chapter 4).

\subsubsection{Final rotation rate $\omega_{\mathrm{f}}$}

It turns out that, for the planets of interest in this Section, the final rotation rate $\omega_{\mathrm{f}}$ can be safely neglected in eq. (3.7): The synchronous rotation rate $\omega_{\text {orbit }}$ given by Kepler's law, eq. (3.1), is much smaller than the value of $\omega_{\mathrm{i}}$. Similarly to Section 3.1.4, the maximum value of $\omega_{\text {orbit }}$ is computed by:

$$
\omega_{\text {orbit }}^{\max }=\sqrt{\frac{M_{\star}^{\max } G}{d_{\text {min }}^{3}}} .
$$

For the terrestrial planets discussed in this work, $M_{\star}^{\max }=1.0 M_{\odot}$ and $d_{\min }=0.1 \mathrm{AU}$, so that $\omega_{\text {orbit }}$ is limited by

$$
\omega_{\text {orbit }} \leq \omega_{\text {orbit }}^{\max }=6.3 \cdot 10^{-6} \mathrm{~s}^{-1},
$$

corresponding to a rotation period of 277 hours. A comparison to the initial rotation periods of between 10 and 30 hours (see above) shows that this contribution is negligible here. Because $\omega_{\mathrm{f}} \leq 2 \omega_{\text {orbit }}$, the value of $\omega_{\mathrm{f}}$ will be set to zero for the calculation of the tidal locking timescale of terrestrial exoplanets. 


\subsubsection{Overview of the parameters}

Table 3.2 lists the planetary parameters required for the estimation of the tidal locking timescale for terrestrial exoplanets. The planetary mass $M_{\mathrm{p}}$ and radius $R_{\mathrm{p}}$ are given in units normalised to Earth (denoted by subscript E), with $R_{\mathrm{E}}=6371 \mathrm{~km}$ (Cain et al. 1995) and $M_{\mathrm{E}}=6.0 \cdot 10^{24} \mathrm{~kg}$ Raith 1997).

\begin{tabular}{|l||c|c|c|c|c|c|c|c|}
\hline Planet & $\alpha$ & $Q_{\mathrm{p}}$ & $k_{2, \mathrm{p}}$ & $Q_{\mathrm{p}}^{\prime}$ & $R_{\mathrm{p}}\left[R_{\mathrm{E}}\right]$ & $M_{\mathrm{p}}\left[M_{\mathrm{E}}\right]$ & $\omega_{\mathrm{i}}\left[\omega_{\mathrm{E}}\right]$ & $\omega_{\mathrm{f}}$ \\
\hline \hline Earth & $0.33^{a}$ & $12^{b}$ & $0.3^{b}$ & 60 & 1.0 & 1.0 & $0.80 \ldots 1.83$ & 0 \\
Ancient Earth & $0.33^{c}$ & $100^{d}$ & $0.3^{c}$ & 500 & 1.0 & 1.0 & $0.80 \ldots 1.83$ & 0 \\
Mercury & $0.33^{c}$ & $100^{b}$ & $0.1^{b}$ & 1500 & $0.38^{e}$ & $0.055^{f}$ & $0.80 \ldots 1.83$ & 0 \\
Large Earth & $0.33^{c}$ & $100^{d}$ & $0.3^{c}$ & 500 & $1.63^{g}$ & $6.0^{g}$ & $0.80 \ldots 1.83$ & 0 \\
Ocean Planet & $0.33^{c}$ & $100^{d}$ & $0.3^{c}$ & 500 & $2.0^{g}$ & $6.0^{g}$ & $0.80 \ldots 1.83$ & 0 \\
\hline
\end{tabular}

Table 3.2: Parameters for the calculation of the tidal locking timescale for different terrestrial planets. Planetary masses and radii are given in units normalised to Earth. Sources of the parameters: ${ }^{(a)}$ Goldreich and Soter (1966), ${ }^{(b)}$ Murray and Dermott (1999), ${ }^{(c)}$ assumed in analogy to Earth, ${ }^{(d)}$ assumed in analogy to Venus, ${ }^{(e)}$ Cain et al. (1995), ${ }^{(f)}$ Raith (1997), ${ }^{(g)}$ Léger et al. (2004).

\subsubsection{Results for terrestrial planets}

Here, the tidal locking timescale is calculated for terrestrial exoplanets (as defined in Section 2.2.5. It is shown that terrestrial exoplanets within the habitable zone of low mass (M type) stars are likely to be synchronously rotating.

Again, as it was done for Hot Jupiters in Section 3.1.4, the timescale for tidal locking of terrestrial planets is calculated using eq. (3.7). Subsequently, the planets can be classified as either "tidally locked", "potentially tidally locked" or "unlocked". The upper and lower boundaries of the potentially locked region are determined by the conditions $\tau_{\text {sync }}=100 \mathrm{Myr}$ and $\tau_{\text {sync }}=10 \mathrm{Gyr}$, respectively. To account for the uncertainty of the initial rotation rate $\omega_{i}$, the lower boundary is calculated with $\omega_{i}=1.83 \omega_{\mathrm{E}}$ (i.e. a rotation period of $13.1 \mathrm{~h}$ ), and the upper boundary is calculated with $\omega_{\mathrm{i}}=0.80 \omega_{\mathrm{E}}$ (corresponding to a rotation period of $30 \mathrm{~h}$ ), see Table 3.2. This increases the area of the "potentially locked" region.

The different classes are best examined in the $d-M_{\star}$-plane, keeping the planetary parameters $Q_{\mathrm{p}}, R_{\mathrm{p}}$, and $M_{\mathrm{p}}$ fixed. This is done for an Earth-like planet in Fig. 3.5. The left part of the diagram (from the left border of the diagram to the solid line) contains the points in the $d$ - $M_{\star}$-parameter space where tidal locking occurs very quickly $\left(\tau_{\text {sync }} \leq 0.1\right.$ Gyr). All planets that will be discovered at such orbital distances will be synchronously rotating. For all planets right of the dashed line the synchronisation timescale is very long $\left(\tau_{\text {sync }} \geq 10 \mathrm{Gyr}\right)$. Any planet observed in this regime will not have lost much of its initial angular momentum. The region between the solid and the dashed line corresponds to systems in which an observed planet may or may not be tidally locked $\left(0.1 \mathrm{Gyr} \leq \tau_{\text {sync }} \leq 10\right.$ 


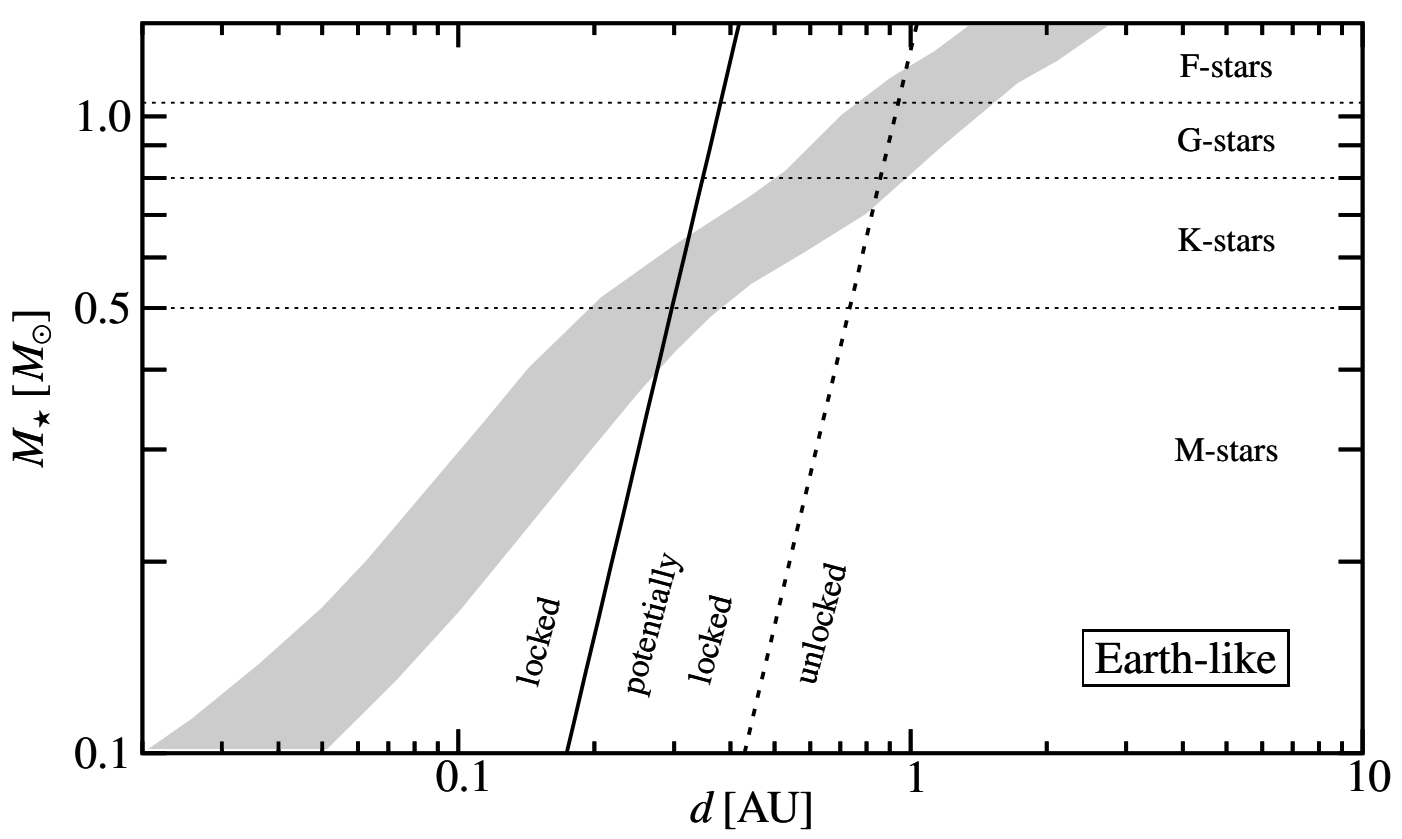

Figure 3.5: Tidal locking regimes for an Earth-like planet as a function of orbital radius $d$ and mass $M_{\star}$ (or spectral type) of the host star. The shaded area gives the location of the habitable zone (see text).

Gyr).

In addition, the extension of the "habitable zone" as a function of stellar mass is shown in Fig. 3.5 (shaded area). The habitable zone around a star is the region in which liquid water can exist on the planetary surface (Kasting et al. 1993). Strictly speaking, the size and location of the habitable zone does not only depend on the size of the star, but also, among other factors, on the planetary atmospheric composition, size, mass and radius. Because the planetary parameters only have limited influence on the location and width of the habitable zone, the "continuously habitable zone" (see Section 2.3) as calculated for the Earth by Kasting et al. (1993) will be used throughout this Section. With Fig. 3.5 it is thus possible to determine whether potentially habitable planets are tidally locked.

In addition to the current Earth, four additional configurations are examined:

- As the tidal dissipation factor $Q_{\mathrm{p}}$ is believed to have been larger in the past, the case of the Earth is studied for an increased value of $Q_{\mathrm{p}}$, see Section 3.1.5.2. Fig. 3.6 shows that this reduced efficiency of the tidal interaction slightly decreases the parameter range of the "tidally locked" regime. While the difference is not very large, it seems possible to find such (modified) Earth-like planets which are not totally locked around heavy M stars (i.e. with $M_{\star} \approx 0.5 M_{\odot}$ ). This case is relevant for two different scenarios: a) it probably applies to the early history of the Earth, and may thus be relevant for Earth-like planets during the early stages of planetary evolution, and b) it is relevant for planets identical to the Earth only in mass and size, but without Earth-like oceans.

- The case of a much smaller, Mercury-like planet is studied. Because the average 


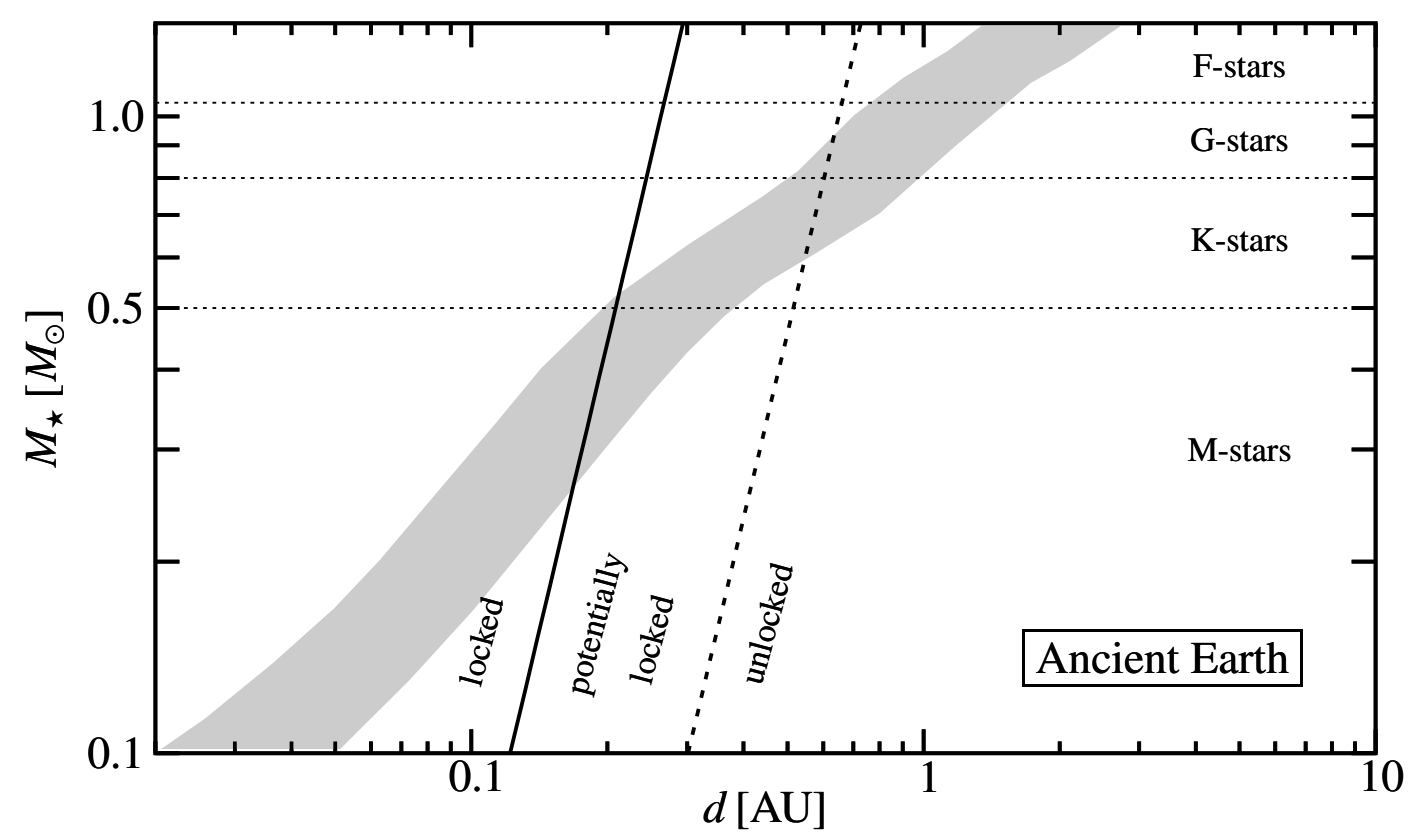

Figure 3.6: Tidal locking regimes for an Earth-like planet with increased value of $Q_{\mathrm{p}}$ as a function of orbital radius $d$ and mass $M_{\star}$ (or spectral type) of the host star. The shaded area gives the location of the habitable zone (see text).

density of Mercury is similar to that of the Earth, the difference in the tidal locking timescales mainly stems from the different value of $Q_{\mathrm{p}}$. Figure 3.7 shows that any terrestrial planet with a value of $Q_{\mathrm{p}}$ as large as that of Mercury around a K or M star is tidally locked for any orbital distance $\leq 0.1 \mathrm{AU}$. For orbital distances in the range $0.1 \mathrm{AU} \leq d \leq 0.2 \mathrm{AU}$, the planet is tidally locked, if the stellar mass is large enough. Partial tidal locking around a $\mathrm{K}$ star is possible up to distances of $0.5 \mathrm{AU}$.

- The opposite case of a large terrestial planet is depicted in Fig. 3.8. Because the average density is similar to the "Ancient Earth" case and the other parameters are identical, the result is very similar to that case (see Fig. 3.6, and above).

- The same is true for the case of an Ocean Planet, see Fig. 3.9.

These results can be compared to the solar system: Mercury has a semi-major axis of 0.39 AU (Murray and Dermott 1999), which, according to Fig. 3.7. puts the planet into the "potentially locked" regime. In fact, Mercury is in a 3:2 spin-orbit resonance, i.e. it rotates three times about its axis during two full orbits around the Sun (Hubbard 1984, Chapter 4). This is only possible for planets in eccentric orbits. Venus has a semi-major axis of $0.72 \mathrm{AU}$, and the parameters are similar to those of the "Ancient Earth" case. In Fig. 3.6, this corresponds to the unlocked case. As suggested by Hubbard (1984), this may point towards stronger dissipation in the past or towards an overestimation of the current value of $Q_{\mathrm{p}}$ : A value of $Q_{\mathrm{p}} \leq 17$ would be required to explain spin-down to the current state, see Goldreich and Soter (1966). The situation of Venus is further complicated by the presence of thermal atmospheric tides, as was discussed in Section 3.1.2. All other planets of the solar system are far from being tidally locked. 


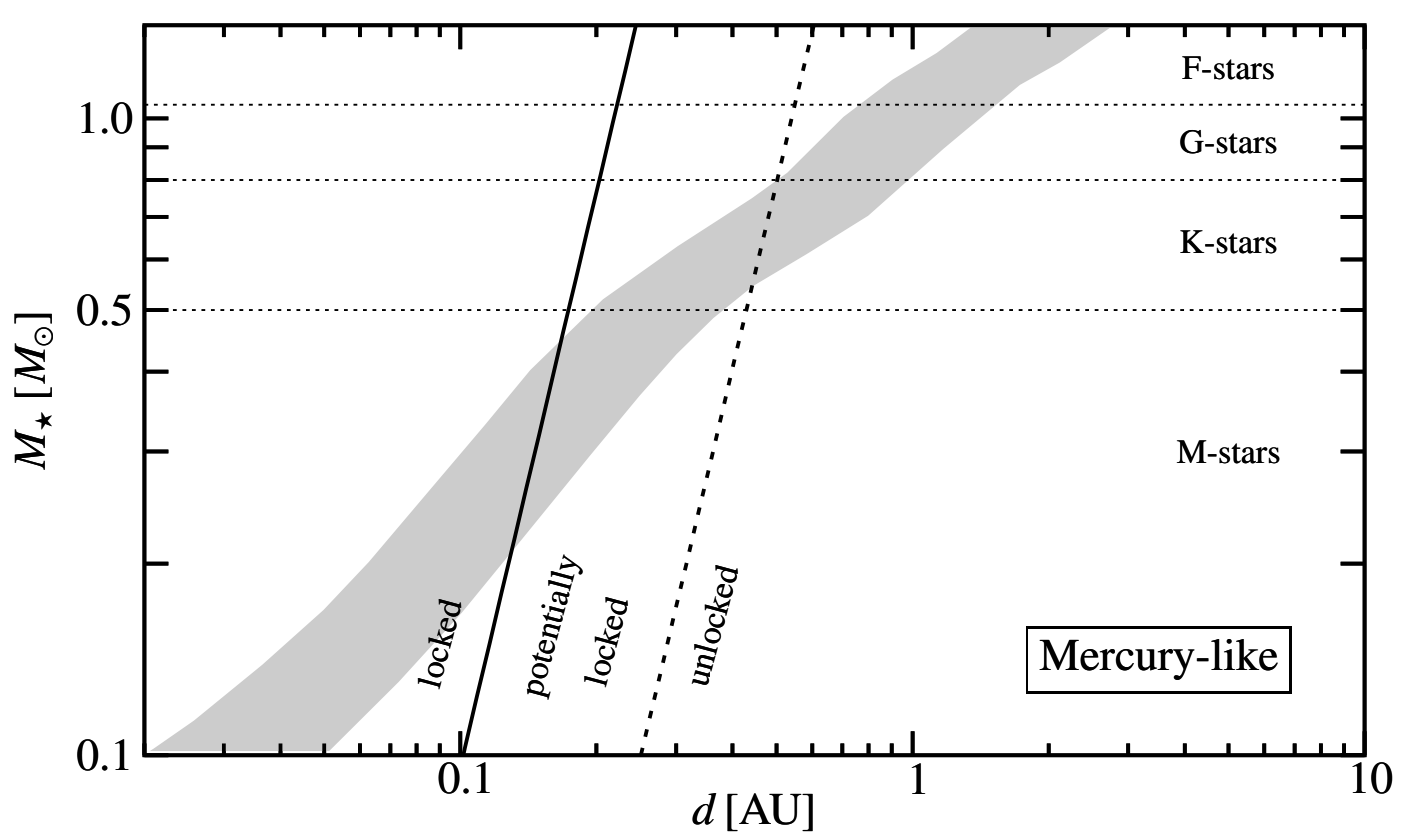

Figure 3.7: Tidal locking regimes for a Mercury-like planet as a function of orbital radius $d$ and mass $M_{\star}$ (or spectral type) of the host star. The shaded area gives the location of the habitable zone (see text).

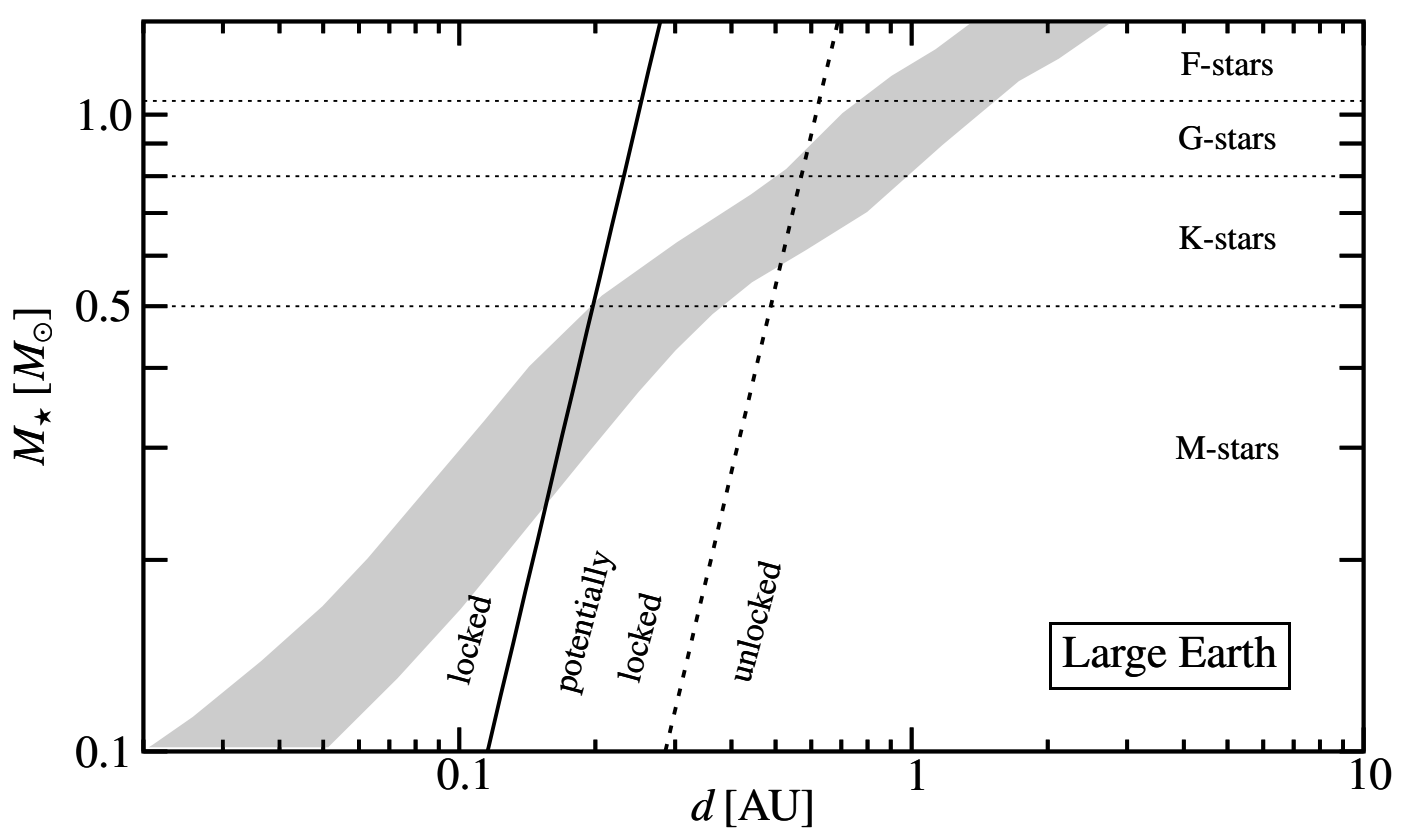

Figure 3.8: Tidal locking regimes for a large terrestrial planet as a function of orbital radius $d$ and mass $M_{\star}$ (or spectral type) of the host star. The shaded area gives the location of the habitable zone (see text).

As a typical test case, a terrestrial exoplanet at an orbital distance of $0.2 \mathrm{AU}$ around a 


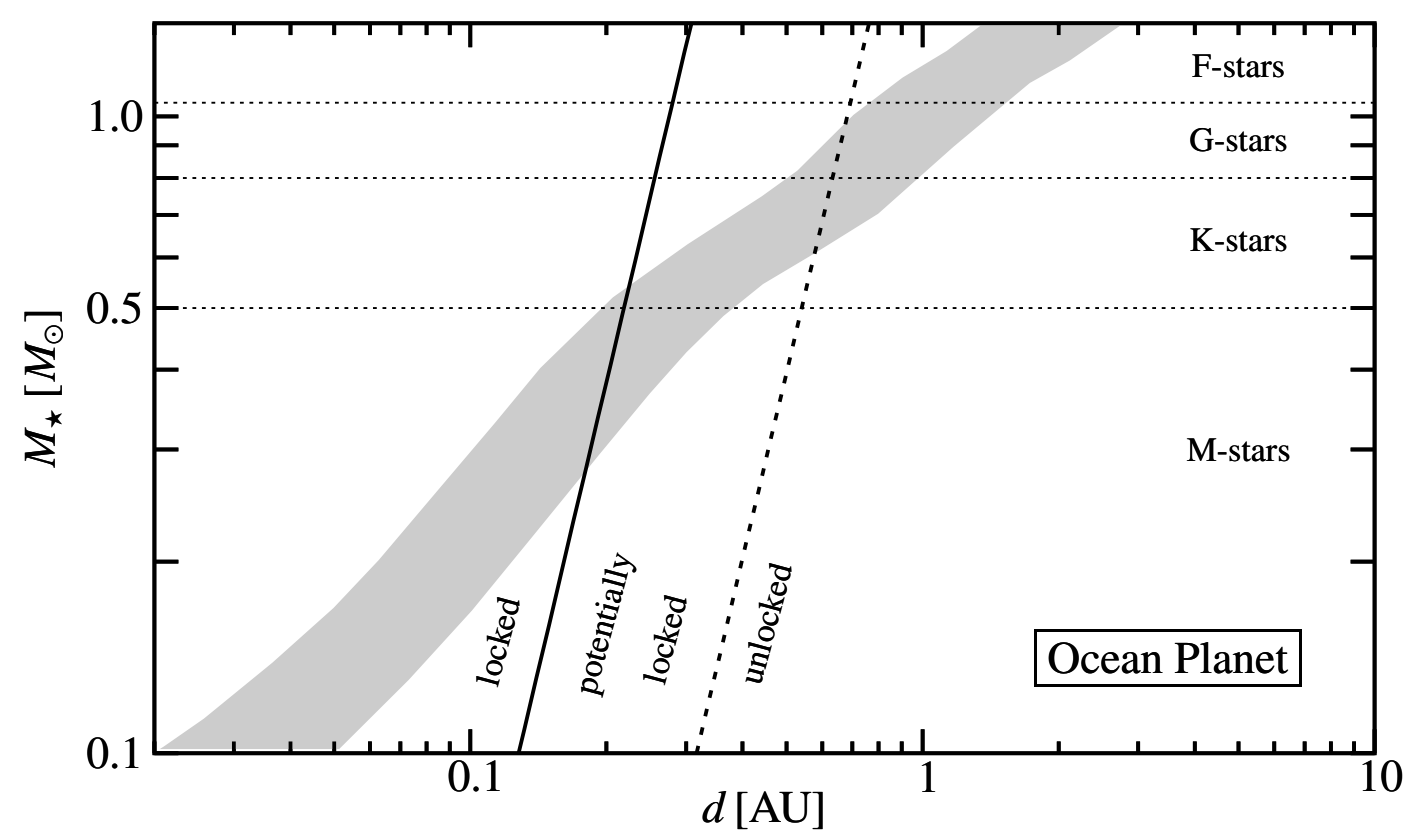

Figure 3.9: Tidal locking regimes for an Ocean Planet as a function of orbital radius $d$ and mass $M_{\star}$ (or spectral type) of the host star. The shaded area gives the location of the habitable zone (see text).

star of $0.5 M_{\odot}$ will be studied in this work. Figs. 3.5 to 3.9 clearly show that such a planet, regardless of its precise mass, radius, and composition, is very likely to be tidally locked. Although its orbital distance places such a planet inside the habitable zone, the reduced rotation rate of tidally locked planets is supposed to have important consequences for the planetary magnetic dipole moment, which in turn could have important implications on the planetary habitability. These issues will be discussed in Section 4 and in Section 7 .

\subsection{Orbital circularisation}

Close-in giant planets are usually found in orbits with low eccentricities. Halbwachs et al. (2005) found a clear separation between circularised and eccentric orbits with orbital periods below and above the "cutoff" orbital period $P_{\text {cutoff }}$ of 5 days, respectively. Using analytical estimations, Gu et al. (2003) find that Jupiter-like planets are circularised within the main sequence lifetime of solar type stars if the orbital period is less than one week.

As will be discussed in section 3.3, circular orbits are a necessary prerequisite for a simple treatment of obliquity damping. This section will briefly discuss why and when close-in giant planets and terrestrial exoplanets can be expected to be circularised by tides. Note that circular orbits are possible also outside this regime (orbital circularisation is a sufficient, but not a necessary condition for circular orbits).

As far as the eccentricity evolution is concerned, two effects have to be taken into account (Goldreich and Soter 1966, Peale 1999):

- Tidal dissipation of energy in the secondary body (in this case, the planet), tends 
to reduce the eccentricity $e$. This effect can be understood by considering that the energy is not conserved, while the angular momentum remains constant. For this reason, the semi-major axis $d$ (which depends on the total energy) may change, while the quantity $d\left(1-e^{2}\right)$ (the "semilatus rectum", which is a function of the angular momentum) is conserved. Thus, when energy dissipation forces the semimajor axis $d$ to decrease, the eccentricity $e$ has to be diminished, too. When the primary star is a slow rotator, the radius of the final circular orbit becomes identical to the semilatus rectum (Halbwachs et al.2005).

- Dissipation within the primary object (in this case, the star) tends to increase the eccentricity of the secondary body. The greater tidal force on the secondary during the close approach at the periapsis tends to send it to a greater apoapse distance, which increases the eccentricity $e$ (Peale 1999).

As will be seen in the following, the second effect can be neglected if the star is not spinning too rapidly. For not too high eccentricity, the critical stellar rotation rate $\omega_{\star}^{\text {crit }}$, for which stellar tides become important, is given by (Gu et al. 2003, Dobbs-Dixon et al. 2004):

$$
\omega_{\star}^{\text {crit }}=\left[\frac{18}{11}+\frac{7}{11} \frac{Q_{\star}^{\prime}}{Q_{\mathrm{p}}^{\prime}}\left(\frac{M_{\star}}{M_{\mathrm{p}}}\right)^{2}\left(\frac{R_{\mathrm{p}}}{R_{\star}}\right)^{5}\right] \omega_{\text {orbit }} .
$$

For stellar spin rates above this value, the influence of the stellar tides dominates, whereas for $\omega_{\star} \ll \omega_{\star}^{\text {crit }}$, this contribution can be neglected. $Q_{\star}^{\prime}$ denotes the (modified) stellar tidal dissipation factor defined in analogy to the planetary tidal dissipation $Q_{\mathrm{p}}^{\prime}$. Its value is not known precisely; using $Q_{\star}$ and $k_{2, \star}$ from Pätzold et al. (2004) and eq. (3.5), one obtains values in the range of $9 \cdot 10^{6} \leq Q_{\star}^{\prime} \leq 2 \cdot 10^{9}$. The synchronous spin rate of the planet, $\omega_{\text {orbit }}$ is given by eq. (3.1):

$$
\omega_{\text {orbit }}=\sqrt{\frac{G M_{\star}}{d^{3}}} .
$$

With the stellar parameters taken from the Sun $\left(M_{\star}=M_{\odot}=1.98911 \cdot 10^{30} \mathrm{~kg}\right.$ and $R_{\star}=R_{\odot}=6.96 \cdot 10^{8} \mathrm{~m}$, see Murray and Dermott 1999), eq. 3.12 yields a value of $7 \cdot 10^{1} \omega_{\text {orbit }} \leq \omega_{\star}^{\text {crit }} \leq 2 \cdot 10^{4} \omega_{\text {orbit }}$ for a Jupiter-like planet (using the parameters of Section 3.1.3 and $8 \cdot 10^{4} \omega_{\text {orbit }} \leq \omega_{\star}^{\text {crit }} \leq 2 \cdot 10^{8} \omega_{\text {orbit }}$ for an Earth-like planet (using the parameters of Section 3.1.5). With orbital periods of the order of a few days, one finds $\omega_{\star} \ll \omega_{\star}^{\text {crit }}$ for all except very young stars, regardless of the planet involved. Thus, the second contribution to the orbital evolution of extrasolar planets can be neglected.

In such a case the timescale $\tau_{\text {circ }}$ for damping of the orbital eccentricity $e$ to zero is given by (Goldreich and Soter 1966, Gu et al. 2003, Bodenheimer et al. 2003):

$$
\tau_{\text {circ }}=-\frac{e}{\dot{e}}=\left(\frac{4}{63}\right)\left(\frac{Q_{\mathrm{p}}^{\prime}}{\omega_{\text {orbit }}}\right)\left(\frac{M_{\mathrm{p}}}{M_{\star}}\right)\left(\frac{d}{R_{\mathrm{p}}}\right)^{5}=\left(\frac{4}{63}\right)\left(\frac{Q_{\mathrm{p}}^{\prime}}{\sqrt{G}}\right)\left(\frac{M_{\mathrm{p}}}{M_{\star}^{3 / 2}}\right)\left(\frac{d^{13 / 2}}{R_{\mathrm{p}}^{5}}\right) .
$$

Here, $Q_{\mathrm{p}}^{\prime}$ is the (modified) planetary tidal dissipation factor, $G$ is the constant of gravity, $M_{\mathrm{p}}$ and $M_{\star}$ denote the planetary and stellar mass, respectively, $d$ is the semi-major axis of the orbit, and $R_{\mathrm{p}}$ is the planetary radius. Besides the vanishing contribution of stellar tides (discussed above), the derivation of eq. (3.13) assumes that the eccentricity $e$ is not too large ( $e \lesssim 0.2$ for an error smaller than a factor of two in $\tau_{\text {circ }}$ ). Furthermore, both 
synchronous rotation (discussed in Section 3.1) and the alignment of the planetary spin axis with the orbit are assumed. The more general case of an arbitrary planetary rotation rate and arbitrary orbital eccentricity, as well as the case of a rapidly spinning star are discussed by Dobbs-Dixon et al. (2004).

Figure 3.10 compares the timescale for orbital circularisation ( $\tau_{\text {circ }}$, lines with symbols) from eq. (3.13) to the timescale for synchronous rotation $\left(\tau_{\text {sync }}\right.$, lines without symbols) from eq. (3.7). As in Section 3.1, the solid lines denote a constant timescale of 0.1 Gyr. To the left of these lines, the timescale is even shorter, so that tidal locking and circularised orbits may be assumed, respectively. For planets on a dashed line, the corresponding timescale is equal to $10 \mathrm{Gyr}$. All planets detected with parameters which fall to the right of such a curve are unaffected by the respective tidal effect. For Jupiter, the solid lines were calculated with $Q_{\mathrm{p}}^{\prime}=10^{6}$, and the dashed lines with $Q_{\mathrm{p}}^{\prime}=10^{5}$. See Sections 3.1.3 and 3.1.5 for all other parameters.

The results are the following: For Hot Jupiters around Sun-like stars, circularised orbits can be assumed if $d \leq 0.03 \mathrm{AU}$, see Figure 3.10(a). For values $0.03 \mathrm{AU} \leq d \leq$ $0.1 \mathrm{AU}$, partial circularisation can be expected. This approximately corresponds to the cutoff orbital period of 5 days found by Halbwachs et al. (2005), which, for a Sun-like star, corresponds to an orbital distance of 0.06 AU. Figure 3.10 also shows that orbital circularisation occurs on a much longer timescale than tidal locking, so that the critical orbital distances $d$ are much smaller.

For Earth-like exoplanets, Figure 3.10(b) shows that orbital circularisation occurs for larger orbital distances than for Hot Jupiters. This is caused by the stronger tidal dissipation in the solid bodies (i.e. smaller values of $Q_{\mathrm{p}}^{\prime}$ ). However, for planets within the habitable zone (defined in Section 2.3), planets in eccentric orbits are likely to exist. As for Hot Jupiters, the timescale for orbital circularisation is much larger than that for tidal locking, so that the critical orbital distances $d$ are much smaller.

These results can be compared to the solar system: With a semi-major axis of $0.39 \mathrm{AU}$, Mercury is in the "not circularised" regime, see Fig. 3.11. Observationally, Mercury has an eccentricity of $e=0.206$ (Hubbard 1984). All other planets of the solar system are even further away from orbital circularisation.

Figure 3.10 shows that both for a) Hot Jupiters and b) the terrestrial test planet at 0.2 AU around a 0.5 solar mass star, eccentric orbits cannot be ruled out. While for Hot Jupiters some tidal influence on the orbital eccentricity can be expected and is consistent with observations (Halbwachs et al. 2005), no such conclusion is possible for the terrestrial planets considered in this work. Nonetheless, the remainder of this work (with the exception of Section 3.3 will concentrate on planets on circular orbits to avoid unnecessary complications.

\subsection{Obliquity damping}

In Section 5.3.1, a model of the planetary magnetosphere will be constructed under the assumption that the magnetic moment is perpendicular to the orbital plane of the planet. The planetary magnetic moment is taken to be parallel to the planetary rotation axis, and will be estimated in Section 4. Thus, the magnetospheric model implicitly relies on the assumption that the planetary rotation axis is perpendicular to the orbital plane. For this 


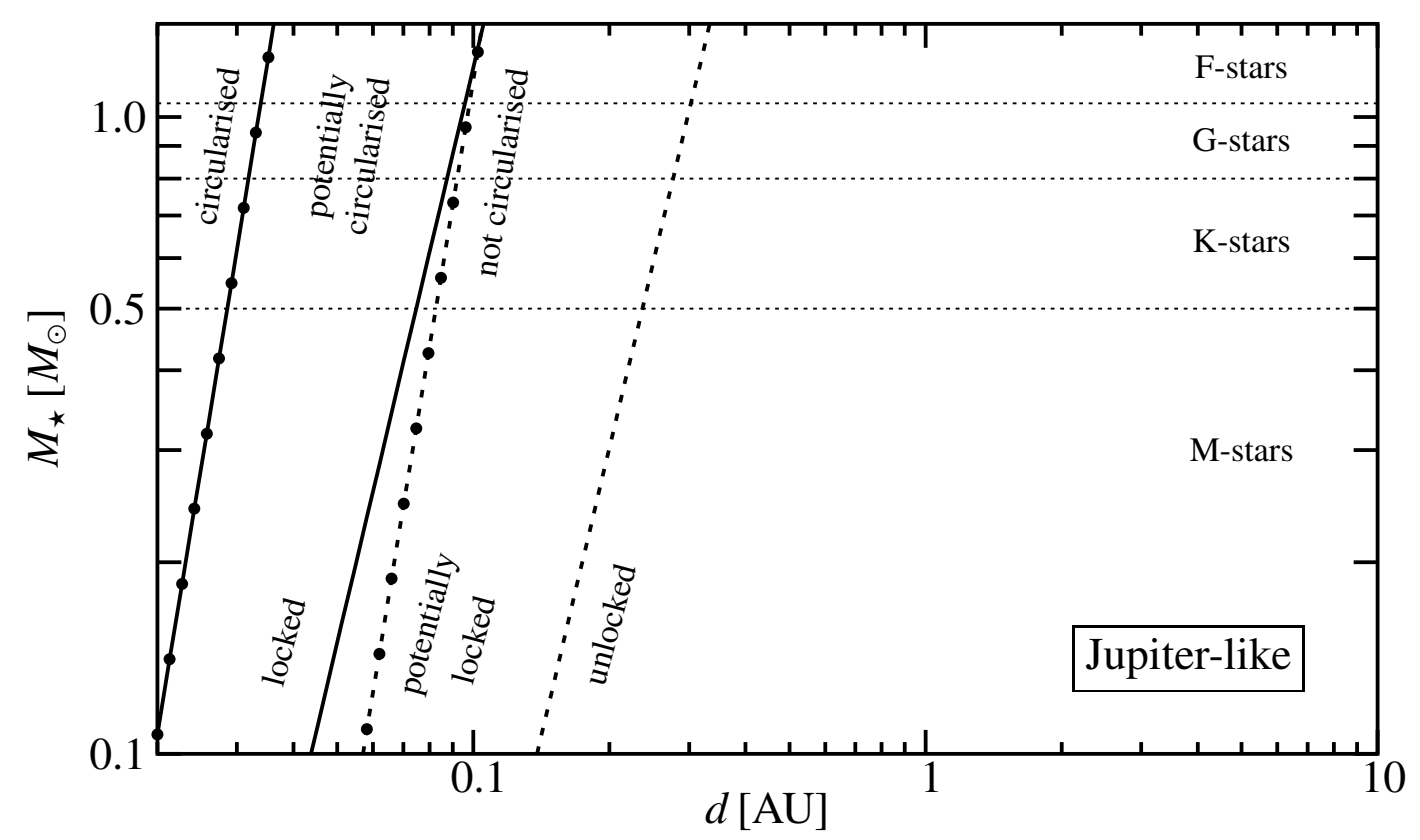

(a) Parameter regimes for a Jupiter-like planet.

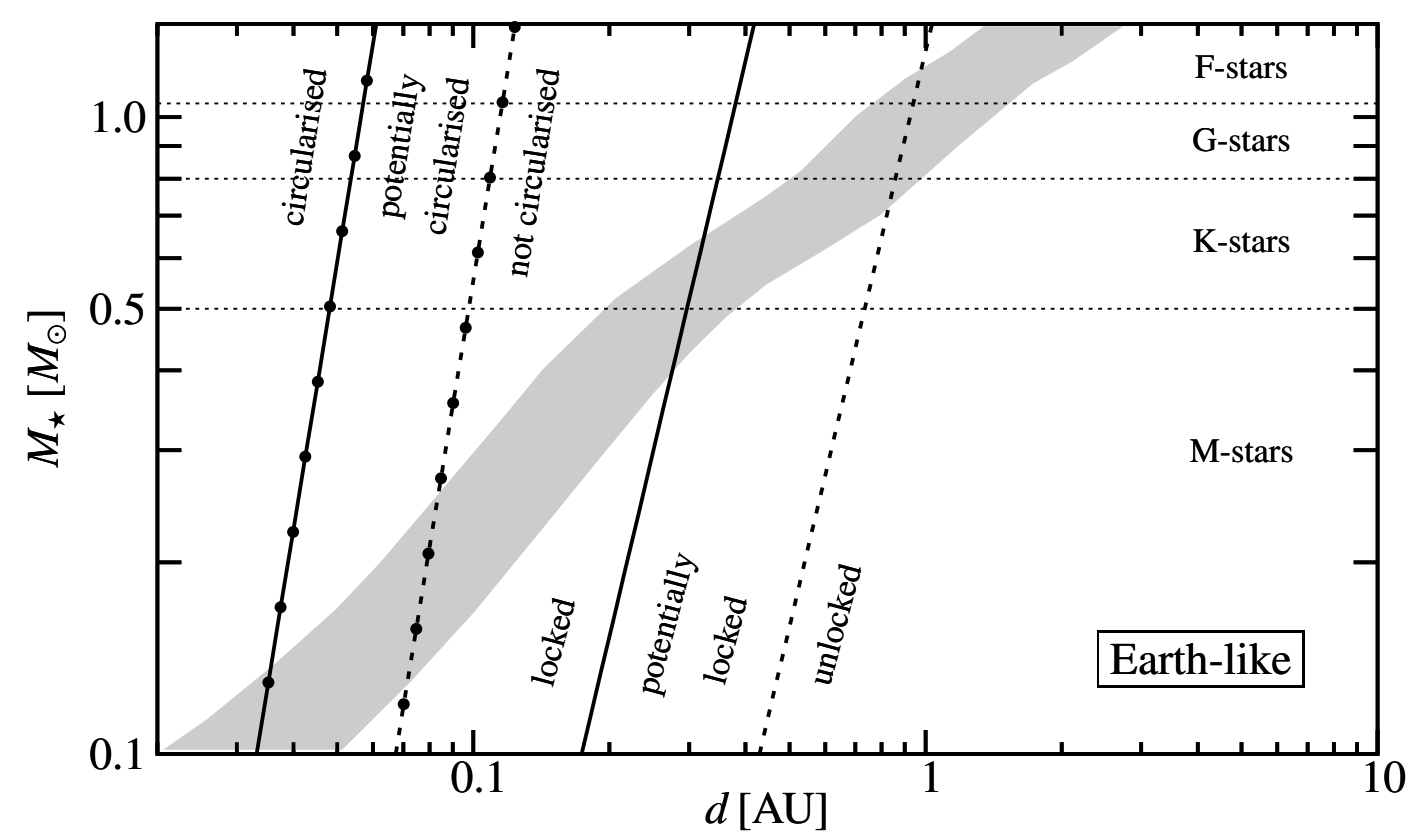

(b) Parameter regimes for an Earth-like planet.

Figure 3.10: Parameter regimes for orbital circularisation (lines with symbols) and for tidal locking (lines without symbols) as a function of orbital radius $d$ and mass $M_{\star}$ (or spectral type) of the host star. The shaded area gives the location of the habitable zone (see Section 2.3.

reason, it is important to check the inclination of the planetary rotation axis with respect to the normal vector of the orbital plane. This important quantity is referred to as the 


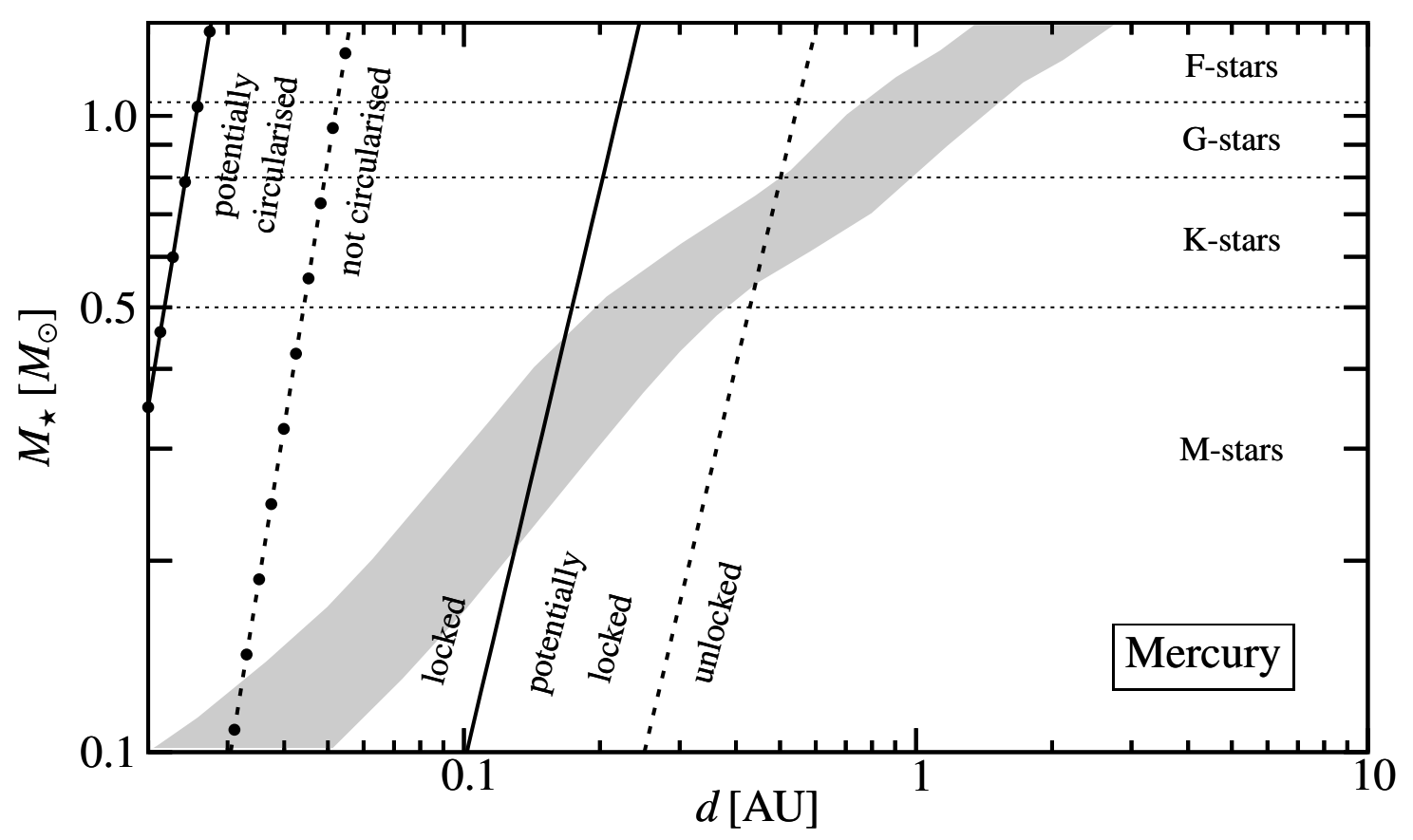

Figure 3.11: Parameter regimes for a Mercury-like planet as a function of orbital radius $d$ and mass $M_{\star}$ (or spectral type) of the host star. The shaded area gives the location of the habitable zone (see Section 2.3).

obliquity. If the obliquity is zero, the planetary magnetic field can be expected to be a zonal dipole (similar to the magnetic topology of Jupiter and Saturn). For non-zero obliquity, however, the rotation might give rise to different, more complicated magnetic configurations like a pole-on dipole.

In the case of tidally locked planets in circular orbits, the assumption of zero obliquity is valid. It can be shown that planets on circular orbits have identical timescales for tidal locking and obliquity damping. The following argumentation was taken from Peale (1999): The planetary spin vector consists of two components, one parallel and one perpendicular to the orbit normal. While the parallel component is tidally damped throughout the planetary orbit, the perpendicular component is not damped when it points towards (or away from) the central body. Averaged over the planetary orbit, the perpendicular component experiences less damping than the parallel component, so that the obliquity increases until it reaches an equilibrium state. This equilibrium state is close to $90^{\circ}$ for rapidly spinning planets, but it decreases towards $0^{\circ}$ when the planetary rotation is reduced. When the planetary rotation approaches synchronous rotation, the equilibrium obliquity is $0^{\circ}$, so that tidal locking and zero obliquity are reached simultaneously (Peale 1999). In other words, the rotation axis of tidally locked planets in circular orbits will be parallel to the orbit normal. If the orbit is not circular, but eccentric, the obliquity evolution is complex, and coupled to other orbital evolution timescales (Seager and Hui 2002).

In the following, the two cases of Hot Jupiters and terrestrial planets are treated separately:

- For Hot Jupiters with small orbital distances $(d \lesssim 0.05 \mathrm{AU})$, it was shown in section 
3.2 that circular orbits are confirmed both by theory and observation. In these cases, zero obliquity can be expected

- For close-in terrestrial planets at orbital distances $d \gtrsim 0.1 \mathrm{AU}$, however, eccentric orbits cannot be ruled out (Section 3.2). Thus, large values for the planetary obliquity cannot be excluded from the viewpoint of tidal interaction. On the timescale of a few Gyr, however, not only tidal dissipation, but also core-mantle friction has to be taken into account (Correia et al.|2003). This eventually drives the planet into one of the two stable states: either the obliquity is $0^{\circ}$ or $180^{\circ}$. This is true regardless whether a planetary atmosphere is present or not (Correia et al. 2003). However, the time required to reach this final state depends on many parameters. For example, a very strong dependence on the initial rotation rate is found in the numerical simulations of Venus by Correia and Laskar (2003b). For high initial rotation rates (corresponding to rotation periods of one day or less), Correia and Laskar (2003b) find obliquity damping times of the order of $10 \mathrm{Gyr}$, which suggests that either the initial rotation period of Venus was not that high, or that the value of $Q_{\mathrm{p}}$ was overestimated by Correia and Laskar (2003b).

Thus, for terrestrial exoplanets at orbital distances of approximately $0.2 \mathrm{AU}$, neither tidal interaction nor core-mantle friction are strong enough to damp away the planetary obliquity on a short enough timescale to allow the assumption of zero obliquity for all planets. Nonetheless, the discussion in this work will focus on those planets that have zero obliquity, postponing the treatment of other configurations to future studies.

\footnotetext{
6 The obliquity of a Jupiter-like planet may even be accessible to measurements: The light curve of a transiting planet can be asymmetric with respect to the ingress/egress, i.e. the phases when only part of the planet is blocking stellar light. However, such an asymmetry requires that the planet has nonzero oblateness and nonzero obliquity (Seager and Hui 2002). For oblate planets, this provides a method to measure the planetary obliquity together with the rotation rate. Unfortunately, Hot Jupiters are not expected to be significantly oblate because of their the low rotation (due to tidal locking, see Section 3.1). Also, Hot Jupiters are not expected to be oblique, see above. For this reason, this technique is more useful for planets further away from their host star.
} 


\title{
4 Planetary magnetic moments
}

\author{
Magnus magnes ipse est globus terrestris. \\ William Gilbert, 1544-1603
}

Planetary magnetic fields are generated by a highly complex combination of different hydromagnetic processes. The source of the internal magnetic field is the motion of a highly conductive fluid within the planet (i.e. the liquid outer core for terrestrial planets, or a layer of electrically conducting hydrogen for gas giants).

For the Earth, the motion of the liquid outer core is believed to be caused by a combination of thermal and chemical convection. While thermal convection due to temperature gradients may have dominated in the early stages of the Earth's evolution (Stevenson 1983. Stevenson et al. 1983), at the present stage the growth of the solid inner core is believed to be the dominating driving force for the dynamo process (Stevenson 2003). Due to the gradual cooling of the Earth, material is freezing out at the boundary between the solid inner and the liquid outer core, and chemical fractionation occurs. Chemically driven convection is then caused by the release of latent heat during the growth of the inner core as well as by the release of buoyant light material which remains after the solidification of heavier components, thus setting free gravitational energy (Stevenson 1983, Stevenson et al. 1983).

For Jupiter, a continuous transition between a layer of fluid monoatomic metallic hydrogen and a less conductive layer of fluid diatomic molecular hydrogen is expected (Guillot 1999, Nellis 2000). Because of the gradual change in conductivity, the Jovian magnetic field can be generated within a sphere larger than the phase of pure metallic hydrogen (Smoluchowski 1975, Stevenson 1982). Thermal convection is caused by the gradual cooling of the planetary interior (Stevenson 1983).

The case of the "ice giants" Uranus and Neptune is less well understood. These planets are supposed to have an "ionic ocean" of $\mathrm{H}_{3} \mathrm{O}^{+} \mathrm{OH}^{-}$, which is likely to be convective (Stevenson 1983). It seems probable that the conductivity of water at high pressure is large enough in this region to allow a dynamo to operate (Stevenson 2003). As far as the large tilt of the dipole axis (Connerney 1993) is concerned, it is known that axial flows can sustain non-axial magnetic fields (Holme 1997). The results of Holme (1997) seem to indicate that the magnetic fields of Uranus and Neptune are generated by flows with weaker differential rotation than the fields of the planets Earth, Jupiter and Saturn.

In addition to the internal magnetic field, current systems within the planetary magnetosphere create additional (external) contributions to the total magnetic field. At some locations, these external contributions can be comparable to the internal ones.

Understanding the planetary dynamo in general, the "geodynamo" in particular and the exact nature of the external contributions is a major scientific goal (combining the- 
oretical, numerical and experimental efforts). Because this is not the central topic of this dissertation, a highly simplified approach will be taken. In this section, the planetary dipole moment is estimated from planetary quantities. Higher multipoles, as well as secular variations of magnetic moments, are beyond the scope of this treatment.

For more detailed information on dynamo processes, the reader is referred to the works of Stevenson (1983) and Roberts and Glatzmaier (2000). Stevenson (1983) also reviews different non-dynamo sources for the planetary magnetic moment and shows that none of those can be responsible for the observed fields. Information on the magnetic fields of terrestrial planets is given by Stevenson et al. (1983) and Russell (1993). Observations of the magnetic fields of the outer planets are analysed by Connerney (1993). External contributions to the total magnetic field are discussed by Jordan (1994) and Kertz (1995, Chapter 19), while the influence of some of these contributions on the magnetospheric structure is discussed from the observational and theoretical point of view by Wolf (1995) and Voigt (1995, Section 4), respectively.

This chapter is organised as follows: Section 4.1 introduces and compares the different scaling laws for planetary magnetic dipole moments. The validity and limitations of this approach are reviewed in Section 4.2. In Sections 4.3 and 4.4, the magnetic moment scaling is applied to gas giants: first the different parameters required for the magnetic moment scaling are evaluated (Section 4.3), and in Section 4.4 the results are presented and discussed. The same is then repeated for terrestrial planets in Section 4.5 (required parameters) and Section 4.6 (discussion of results). The external contributions to the total magnetic field created by the magnetosphere are modelled in Section 5.3.1

\subsection{Magnetic moment scaling laws}

Although the exact mechanism responsible for planetary magnetic fields is not fully understood, different attempts were made to find reasonable estimations for the intensity of planetary magnetic dipole moments 1 . Some of these approaches use empirical relations, others are based on theoretical considerations. They all share the common goal to obtain a scaling law (frequently called "magnetic Bode law") relating the planetary magnetic moment to known planetary parameters. Before the various scaling laws obtained by different authors are presented and compared below, some basic and frequently used concepts of magnetohydrodynamics are introduced.

First it has to be noted that the existence of a dynamo seems to be plausible for most planets. One prerequisite for dynamo action to take place is convection of a conducting fluid (Stevenson 1983, 2003). In addition, it is required that the Coriolis force has a large effect on the flow. This condition, however, is easily satisfied, even for the case of the slow rotator Venus (Stevenson 1983, 2003). Thus the question is not whether the planets can sustain a dynamo, but how strong the resulting field will be.

The behaviour of a fluid particle inside a planetary core rotating with angular fre-

\footnotetext{
${ }^{1}$ Note that in the following "planetary magnetic moment" and "planetary (magnetic) dipole moment" are used as synonyms.
} 
quency $\underline{\omega}$ is governed by the following equation of motion (Mizutani et al. 1992):

$$
\begin{aligned}
\rho\left[\frac{\partial \underline{v}}{\partial t}+(\underline{v} \nabla) \underline{v}\right]= & \underbrace{2 \rho \underline{v} \times \underline{\omega}}_{\text {Coriolis force }}+\underbrace{\rho \omega \times(\underline{r} \times \underline{\omega})}_{\text {centrifugal force }}-\underbrace{\nabla p}_{\text {pressure gradient }}+\underbrace{\frac{1}{\mu}(\nabla \times \underline{B}) \times \underline{B}}_{\text {Lorentz force }} \\
& -\underbrace{\rho \nabla \Phi}_{\text {gravity }}+\underbrace{\eta \Delta \underline{v}+\left(\eta+\frac{1}{3} \zeta\right) \nabla(\nabla \underline{v})}_{\text {viscous forces }} .
\end{aligned}
$$

This is the Navier-Stokes equation in a rotating coordinate system. In eq. (4.1), $\rho$ denotes the density of the fluid element, $\underline{v}$ is its velocity and $\underline{r}$ its position, $p$ denotes the pressure, $\mu$ is the permeability, $\underline{B}$ is the magnetic field, $\Phi$ is the gravitational potential, and $\eta$ and $\zeta$ are the constants of viscosity.

Most, but not all scaling laws are based on the comparison of two different contributions to the equation of motion (while neglecting all other contributions). For example, if the Coriolis force is set equal to the contribution of the pressure gradient, one speaks of a "geostrophic balance". If the Coriolis force is balanced by the Lorentz force, the balance is "magnetostrophic".

For the magnetostrophic balance, the first and the fourth term on the right hand side of eq. (4.1) are set equal:

$$
-\underbrace{\frac{1}{\mu}(\nabla \times \underline{B}) \times \underline{B}}_{\text {Lorentz force }}=\underbrace{2 \rho \underline{v} \times \underline{\omega}}_{\text {Coriolis force }} .
$$

The simple comparison of the relevant scales in eq. (4.2) then yields:

$$
\underline{B}^{2}=2 \mu_{0} \rho_{\mathrm{c}} r_{\mathrm{c}} \omega v_{\mathrm{conv}},
$$

where now $\rho_{\mathrm{c}}$ is the density within the dynamo region, $r_{\mathrm{c}}$ is the size of the dynamo region ${ }^{2}$, $v_{\text {conv }}$ is the velocity of the convective motion and $\omega=|\underline{\omega}|$ is the absolute value of the rotation rate.

For the geostrophic balance, the scaling law for the size of the magnetic field can be obtained in the following way (Glaßmeier and Vogt 2001). In eq. (4.1), the first term is assumed to be balanced by the third one. Eq. (4.1) then reduces to

$$
\begin{aligned}
\rho\left[\frac{\partial \underline{v}}{\partial t}+(\underline{v} \nabla) \underline{v}\right]= & \underbrace{\rho \omega \times(\underline{r} \times \underline{\omega})}_{\text {centrifugal force }}+\underbrace{\frac{1}{\mu}(\nabla \times \underline{B}) \times \underline{B}}_{\text {Lorentz force }} \\
& -\underbrace{\rho \nabla \Phi}_{\text {gravity }}+\underbrace{\eta \Delta \underline{v}+\left(\eta+\frac{1}{3} \zeta\right) \nabla(\nabla \underline{v})}_{\text {viscous forces }} .
\end{aligned}
$$

The fluid is assumed to be incompressible (i.e. $\rho=$ const and $\nabla \underline{v}=0$ ). Furthermore, stationarity is assumed (i.e. $\partial \underline{v} / \partial t=\underline{0}$ ), and the contributions of gravitational and centrifugal force are supposed to be negligible. This further simplifies eq. (4.4):

$$
\rho(\underline{v} \nabla) \underline{v}=+\underbrace{\frac{1}{\mu}(\nabla \times \underline{B}) \times \underline{B}}_{\text {Lorentz force }},
$$

\footnotetext{
2 In the following "radius of the dynamo region", "core radius" and "radius of the convection region" are all used as synonyms. For gas giants, this "core" is not to be confused with a potential solid core at the planetary centre.
} 
so that now the Lorentz force is balanced by the inertial force. Again, scale analysis yields the scaling law for the magnetic field intensity:

$$
\underline{B}^{2}=\mu_{0} \rho_{\mathrm{c}} v_{\mathrm{conv}}^{2}
$$

In both the magnetostrophic and the geostrophic case, a dipole field is assumed. Since this implies that the magnetic moment is obtained as $\mathcal{M} \propto B r_{\mathrm{c}}^{3}$, eq. (4.3) and (4.6) lead to the following scaling relations:

$$
\begin{aligned}
\text { magnetostrophic: } & \mathcal{M} \propto \rho_{\mathrm{c}}^{1 / 2} r_{\mathrm{c}}^{7 / 2} \omega^{1 / 2} v_{\text {conv }}^{1 / 2}, \\
\text { geostrophic: } & \mathcal{M} \propto \rho_{\mathrm{c}}^{1 / 2} r_{\mathrm{c}}^{3} v_{\text {conv }} .
\end{aligned}
$$

It can be seen that besides the choice of the force balance, the assumption made about the unknown convection velocity is an important ingredient to any scaling law for the planetary magnetic moment.

The scaling relations for the planetary magnetic field $B$ as obtained by various authors are presented from Section 4.1.1 to Section 4.1.8. These different approaches are compared in Section 4.1.9, where it will also be discussed which scaling laws are used within this work.

\subsubsection{Blackett's law}

The probably oldest scaling law is known as "Blackett's law". Taking up previous ideas, Blackett (1947) suggested that any rotating mass should produce a magnetic field ("modified Schuster-Wilson hypothesis"). The following scaling law resulted:

$$
B \propto \rho_{\mathrm{c}} \omega r_{\mathrm{c}}^{2} .
$$

This theory successfully reproduced the magnetic moments of the Earth, the Sun and of the star 78 Virginis. However, experimental verification of the theory failed. Blackett (1952) showed that magnetic fields of the required intensities could not be generated by rotating masses and withdrew his theory.

Unfortunately, Blackett's scaling law is still regularly cited and applied to predict planetary dipole moments (see the references given by Cain et al. 1995). Because this scaling law has a higher exponent in $r_{\mathrm{c}}$ than all other scaling relations, this is especially problematic for large and heavy planets, see also Section 4.4.

\subsubsection{Busse's geostrophic scaling law}

Busse (1976) discussed a theory based on convection to describe planetary magnetism. In this theory, the toroidal and poloidal components of the field are of comparable size (Stix 1977, Cain et al. 1995). Busse (1976) predicted the magnetic field of Saturn to be $19 \%$ of that of Jupiter, which was too high by a factor of approximately four (Cain et al. 1995). Stix (1977) compared the then known planetary magnetic moments on the basis of Busse's work and found reasonable agreement. Neubauer (1978) showed that from the work of Busse (1976), the scaling law

$$
B \propto \rho_{\mathrm{c}}^{1 / 2} \omega r_{\mathrm{c}}
$$


can be directly obtained for the magnetic field. Neubauer (1978) also estimated the strength of the magnetic moments of the Galilean moons and of Titan on the basis of eq. (4.10). Using this scaling law, Russell (1979) calculated the magnetic field of Saturn, with a result too high by a factor of $\sim 2.5$ (Russell 1979 , note added in proof).

The scaling of Busse (1976) is based on a geostrophic balance, and implicitly assumes that the convection velocity $v_{\text {conv }}$ scales as the core rotation velocity:

$$
v_{\mathrm{conv}}=\omega r_{\mathrm{c}}
$$

However, it is possible to obtain the same scaling law for a magnetostrophic balance, provided that assumption (4.11) is used for the convection velocity (Curtis and Ness 1986, Mizutani et al. 1992). The reason is that both the geostrophic and the magnetostrophic balance simply rely on a dimensional analysis of the equation of motion. Although in both cases different terms are retained, all terms have the same physical dimension. As long as only one length scale is used, the result cannot depend on the chosen balance.

\subsubsection{Scale analysis by Jacobs}

Without making any assumptions on the driving force for the planetary dynamo, Jacobs (1979) derives a scaling law from a dimensional analysis. The scaling law contains the critical Reynolds number; if, however, the critical Reynolds number can be assumed to be identical for all planets, Jacobs (1979) obtains the same scaling law as used by Busse (1976):

$$
B \propto \rho_{\mathrm{c}}^{1 / 2} \omega r_{\mathrm{c}}
$$

\subsubsection{Stevenson's scaling based on heat flow}

Stevenson (1979) assumes that convection is dominated by the linear Boussinesq convective modes which transport the most heat. The "optimal (magnetic) field" is that for which the heat flux is maximised for a given temperature gradient and rotation rate. Alternatively, one can interprete it as the field for which the temperature gradient is minimised for a given heat flux (Stevenson 1979, 1983). For a given dynamo, the field is unstable until it reaches a stable state at or close to this optimal field, provided that enough energy is available to reach that stable state (Stevenson 1983). According to the size of the magnetic Reynolds number $R_{\text {mag }}=v_{\text {conv }} r_{\mathrm{c}} / \lambda$ (where $\lambda=1 / \mu_{0} \sigma$ is the magnetic diffusivity, and $\sigma$ is the electrical conductivity), two different cases can be distinguished.

In the "dissipative limit" $\left(R_{\text {mag }} \ll 1\right)$, the field is given by

$$
B \propto \rho_{\mathrm{c}}^{1 / 2} \sigma^{-1 / 2} \omega^{1 / 2}
$$

Note that for a dynamo to operate, the necessary condition $R_{\operatorname{mag}} \geq \mathcal{O}(1)$ has to be fulfilled (see, for example, Stevenson 1983, Roberts and Glatzmaier 2000, Stevenson 2003, and references therein). For this reason, the dissipative limit will not be used in this work.

In the "dissipationless limit" $\left(R_{\mathrm{mag}} \gg 1\right)$ the "optimum field" is given by (Stevenson 1979, 1983):

$$
B \propto \rho_{\mathrm{c}}^{1 / 2} v_{0}^{3 / 4} \omega^{1 / 4} r_{\mathrm{c}}^{1 / 4},
$$


where $v_{0}$ is the convection velocity that would be found within the core in the case where both rotation and magnetic field are zero (Stevenson 1979). In eq. (4.14) the Rossby number $R_{0}$, which appears in Stevenson (1979) and Stevenson (1983), was replaced by $v_{0} /\left(\omega r_{\mathrm{c}}\right)$ (Stevenson 1979) To be able to compare this to the other scaling laws, a further assumption on $v_{0}$ is required. While Stevenson (1979, eq. (38)) presents a detailed result for $v_{0}$, it contains several planetary parameters which are not well constrained for extrasolar planets. For this reason, an estimation for $v_{0}$ is required. In the case of no magnetic field and rotation, the convection velocity is determined by the heat flux $E$ of the planet (Stevenson 1979), so that the eq. (4.16) of Curtis and Ness (1986) can be adopted (see Section 4.1 .5 below). Inserting $v_{0} \propto\left(E / \rho_{\mathrm{c}}\right)^{1 / 3}$ into eq. 4 4.14), one obtains:

$$
B \propto \rho_{\mathrm{c}}^{1 / 4} E^{1 / 4} \omega^{1 / 4} r_{\mathrm{c}}^{1 / 4}
$$

The advantages and disadvantages of a scaling law depending on the heat flux $E$ are discussed in Section 4.1.5

The interior magnetic field of the Earth can be decomposed into a toroidal and a poloidal contribution. Then, the results (4.13) and (4.15) are valid for the largest field components, which probably is the toroidal field (Stevenson 1983). It is also found that the latter is probably less than a factor of three larger than the poloidal field (Stevenson 1979).

At the same time, however, Stevenson (1983) voices scepticism concerning the applicability of scaling laws. This point is taken up in Section 4.2 .

\subsubsection{Scaling law of Curtis \& Ness}

Using the magnetostrophic balance previously studied by Eltayeb and Roberts (1970), Curtis and Ness (1986) derived a scaling law which only contains externally observable parameters of the planet. Because information on the planetary core are difficult to obtain, they are replaced by more accessible parameters. The convection velocity $v_{\text {conv }}$ is expressed in terms of the observed heat flux $E$ through the planet:

$$
v_{\text {conv }} \propto\left(\frac{E}{\rho_{\mathrm{c}}}\right)^{1 / 3} .
$$

Together with eq. (4.3), eq. (4.16) results in

$$
B \propto \rho_{\mathrm{c}}^{1 / 3} \omega^{1 / 2} r_{\mathrm{c}}^{1 / 2} E^{1 / 6}
$$

Eq. (4.16) relies on the assumption that the heat flux through the planetary surface originates deep within the planet and is able to drive convection. This is not the case, for example, for heat generated by radioactive elements in outer layers of the planet. For Jupiter, however, energy from radioactive decay can only account for a tiny fraction of the observed heat flux (a fraction of about $5 \cdot 10^{-5}$, see Smoluchowski 1967). In fact, Jupiter emits almost twice as much energy as it receives from the Sun (Stevenson 1982,

\footnotetext{
3 Cain et al. (1995) use $R_{\mathrm{o}} \propto v_{\text {conv }} /\left(\omega r_{\mathrm{c}}\right)$. However, Cain et al. (1995) base their magnetic moment scaling on the work of Sano (1993), which contains a typographical error.
} 
1983). The energy source is believed to be the gradual cooling of the planetary interior (Stevenson 1983), so that this energy is accessible for thermal convection. Similarly, the Earth loses more energy than can be created by radioactive decay in the crust and in the mantle (Roberts and Glatzmaier 2000). Still, the strong contribution of radiogenic heat to the overall heat flux lead Mizutani et al. (1992) to criticise the scaling of Curtis and Ness (1986) at least as far as terrestrial planets are concerned. Also note that the apparent advantage of using the observed heat flux through the planetary surface turns into a disadvantage when extrasolar planets are considered, for which this quantity is not as easily accessible.

Curtis and Ness (1986) also introduced estimations for the size of the planetary core $r_{\mathrm{c}}$ and for the core density $\rho_{\mathrm{c}}$. These estimations are presented and discussed in Section 4.3.2 and in Section 4.3.3, respectively.

\subsubsection{Mizutani's scaling law}

Mizutani et al.(1992) examine how different assumptions for the convection velocity $v_{\text {conv }}$ affect the scaling law resulting from the magnetostrophic balance 4.3 . The convection velocity is assumed to be somewhere in the range of

$$
\frac{1}{\mu_{0} \sigma r_{\mathrm{c}}}<v_{\mathrm{conv}}<r_{\mathrm{c}} \omega
$$

The rotation velocity $r_{\mathrm{c}} \omega$ of the core surface, corresponding to eq. (4.11), is taken as the upper limit for $v_{\text {conv }}$, whereas the lower limit is obtained from the condition that the magnetic Reynolds number $R_{\text {mag }}$ should be larger than unity (Mizutani et al. 1992, Roberts and Glatzmaier 2000, Stevenson 2003). Since in a dynamo $v_{\text {conv }}$ has to be within the range defined by eq. (4.18), Mizutani et al. (1992) argue that the geometric mean

$$
v_{\text {conv }}=\left(\frac{\omega}{\mu_{0} \sigma}\right)^{1 / 2}
$$

between the two extreme values presents a reasonable choice. Together with eq. (4.3), this yields the scaling relation

$$
B \propto \rho_{\mathrm{c}}^{1 / 2} r_{\mathrm{c}}^{1 / 2} \omega^{3 / 4} \sigma^{-1 / 4}
$$

for the magnetic field strength in the core. This scaling law was called ISAS-1 scaling by Mizutani et al. (1992).

Alternatively, if the lower limit for $v_{\text {conv }}$ is inserted into eq. (4.3), i.e. when one assumes

$$
v_{\mathrm{conv}}=\frac{1}{\mu_{0} \sigma r_{\mathrm{c}}}
$$

one obtains

$$
B \propto \rho_{\mathrm{c}}^{1 / 2} \omega^{1 / 2} \sigma^{-1 / 2} .
$$

This scaling is identical to that obtained by Stevenson (1983) for the dissipative case (see Section 4.1.4). This is just a coincidence. Later works sometimes call this scaling the 
ISAS-2 scaling. Note, however, that it is reproduced with typographical errors in several publications.

As noted above (Section 4.1.2), the assumption that the convection velocity is identical to the upper limit of eq. (4.18) leads to the same scaling law as the geostrophic approach of Busse (1976).

According to Mizutani et al. (1992), these scaling laws describe the toroidal field within the core rather than the poloidal field (which is the observable component); however, the ratio between poloidal and toroidal field is assumed to be similar for all planets.

\subsubsection{Sano's scaling law}

Most scaling relations have in common that the toroidal field within the planet is modelled, whereas only the poloidal component is accessible to measurements. To overcome this problem, Sano (1993) decomposes the vector fields $\underline{B}$ and $\underline{v}$ into toroidal and poloidal components (i.e. $\underline{B}_{\mathrm{T}}$ and $\underline{B}_{\mathrm{P}}$, and $\underline{v}_{\mathrm{T}}$ and $\underline{v}_{\mathrm{P}}$, respectively). A magnetostrophic balance is used both for the toroidal and the poloidal components of the equation of motion. According to Sano (1993) the ratio of toroidal and poloidal field needs not be invariant for all planets, but depends on various parameters. Using the likely condition $B_{\mathrm{P}} \leq B_{\mathrm{T}}$, Sano (1993) obtains:

$$
B_{\mathrm{P}} \propto \rho_{\mathrm{c}}^{1 / 2} r_{\mathrm{c}}^{1 / 2} \omega
$$

for the poloidal magnetic field. Note that (as in all other scaling laws) in fact $r_{\mathrm{c}}$ should be replaced by $\left(r_{\text {outer }}-r_{\text {inner }}\right)$ when the planet has a central core not participating in the dynamo process (solid inner core of the Earth, rocky core of giant planets).

Although this ansatz is only strictly valid for axisymmetric systems and thus should not be applicable for the strongly tilted dipoles of Uranus and Neptune (see, e.g. Connerney 1993), the predicted values are still in good agreement with the observations.

\subsubsection{Scaling law based on the Elsasser Number}

In the dynamo regime, the Elsasser number inside the region of field generation is known to be of the order of unity (Roberts and Glatzmaier 2000, Stevenson 2003). The Elsasser number is a measure for the relative strength of Lorentz and Coriolis force. Is is defined as

$$
\Lambda=\frac{\sigma B^{2}}{2 \rho_{\mathrm{c}} \omega},
$$

where $\sigma$ is the electrical conductivity in the core, $B$ is the magnetic field intensity, $\rho_{\mathrm{c}}$ is the fluid density in the core and $\omega$ is the rotation rate. From the condition $\Lambda \approx 1$ one can deduce the magnetic field inside the field generation region. For Earth and Jupiter, $\Lambda \approx 0.3$ (Stevenson 2003, Table 2 ). Note that the condition $\Lambda \approx 1$ corresponds to the case where Lorentz and Coriolis force are of similar magnitude (Roberts and Glatzmaier 2000) and where the convection velocity is given by eq. (4.21). For this reason the scaling law resulting from eq. (4.24) is identical to the second scaling law of Mizutani et al. (1992):

$$
B \propto \rho_{\mathrm{c}}^{1 / 2} \omega^{1 / 2} \sigma^{-1 / 2}
$$


This scaling was used by Sánchez-Lavega (2004) to estimate the magnetic fields inside giant extrasolar planets (i.e. at the top of the conducting layer). By assuming that the magnetic field intensity decreases with the distance as $r^{-3}$ (i.e. the field is assumed to be dipolar in nature), the magnetic field at the planetary surface is obtained.

Sánchez-Lavega (2004) also introduced a different way of estimating the size of the dynamo region $r_{\mathrm{c}}$. This will be discussed in Section 4.3.2.

\subsubsection{Overview over the scaling laws}

For ease of reference, the different scaling laws described from Sections 4.1.1 to 4.1.8 are compared in this section. Scaling laws for the magnetic moment are also compared, e.g., in Cain et al. (1995) and Farrell et al. (1999). Note however, that these publications contain typographical errors and present a less complete overview. The scaling laws (4.9), (4.10), (4.12), 4.13), 4.15), 4.17), 4.20), 4.22), 4.23) and (4.25) were multiplied with $r_{\mathrm{c}}^{3}$ to obtain the magnetic moment $\mathcal{M}$ from the magnetic field (i.e. a dipole field is assumed):

$$
\begin{aligned}
& \mathcal{M} \propto \quad \rho_{\mathrm{c}} \omega r_{\mathrm{c}}^{5} \quad \text { Blackett (1947, 1952) } \\
& \mathcal{M} \propto \quad \rho_{\mathrm{c}}^{1 / 2} \omega r_{\mathrm{c}}^{4} \quad \text { Busse (1976) } \\
& \mathcal{M} \propto \quad \rho_{\mathrm{c}}^{1 / 2} \omega r_{\mathrm{c}}^{4} \quad \text { Jacobs (1979) } \\
& \mathcal{M} \propto \quad \rho_{\mathrm{c}}^{1 / 2} \omega^{1 / 2} r_{\mathrm{c}}^{3} \sigma^{-1 / 2} \quad \text { Stevenson (1983, dissipative case) } \\
& \mathcal{M} \propto \rho_{\mathrm{c}}^{1 / 4} \omega^{1 / 4} r_{\mathrm{c}}^{13 / 4} E^{1 / 4} \quad \text { Stevenson (1983, dissipationless case) } \\
& \mathcal{M} \propto \quad \rho_{\mathrm{c}}^{1 / 3} \omega^{1 / 2} r_{\mathrm{c}}^{7 / 2} E^{1 / 6} \quad \text { Curtis and Ness (1986) } \\
& \mathcal{M} \propto \rho_{\mathrm{c}}^{1 / 2} \omega^{3 / 4} r_{\mathrm{c}}^{7 / 2} \sigma^{-1 / 4} \text { Mizutani et al. (1992, moderate convection) } \\
& \mathcal{M} \propto \rho_{\mathrm{c}}^{1 / 2} \omega^{1 / 2} r_{\mathrm{c}}^{3} \sigma^{-1 / 2} \quad \text { Mizutani et al. (1992, slow convection) } \\
& \mathcal{M} \propto \quad \rho_{\mathrm{c}}^{1 / 2} \omega r_{\mathrm{c}}^{7 / 2} \quad \text { Sano (1993) } \\
& \mathcal{M} \propto \quad \rho_{\mathrm{c}}^{1 / 2} \omega^{1 / 2} r_{\mathrm{c}}^{3} \sigma^{-1 / 2} \quad \text { Sánchez-Lavega (2004) }
\end{aligned}
$$

Here, $\mathcal{M}$ denotes the planetary magnetic dipole moment, $\omega$ is the angular velocity of the planet around its spin axis, $\rho_{\mathrm{c}}$ is the density in the dynamo region, $r_{\mathrm{c}}$ is the radius of the dynamo region ${ }^{4}$, and $\sigma$ is the electrical conductivity in the dynamo region. $E$ is the heat flux caused by heat sources within the planet.

In this work, the following scaling laws will be ignored:

- Eq. (4.26) is not used because it was based on a hypothesis which was later experimentally disproven. As a result, the theory was withdrawn by the author (Blackett 1952), see Section 4.1.1.

- As was discussed in Section 4.1.4, the "dissipative limit" is not relevant for planetary dynamos. For this reason, the scaling law (4.29) is discarded.

\footnotetext{
${ }^{4}$ In some cases, $\left(r_{\text {outer }}-r_{\text {inner }}\right)$ should be preferred, see Sano $(1993)$. However, considering the uncertainties involved in scaling laws, the difference is negligible.
} 
- Eqs. 4.30) and (4.31) are not used because they require knowledge of the heat flux arising from internal sources within the planet. This is an observable quantity in the case of planets in the solar system, but not for extrasolar planets. Because the conditions are highly different for close-in extrasolar giant planets, it is difficult to extrapolate the heat flux arising from sources in the dynamo-region of these planets from solar system data 5

- Eq. (4.28), is not further discussed because it is identical to eq. 4.27).

- Likewise, 4.33 and 4.35) are identical, and it is sufficient to treat one of these. Also note that these scalings are identical to eq. 4.29.

The remaining four scaling relations, i.e. eq. 4.27), (4.32), 4.33) and (4.34) will be taken into account when evaluating the magnetic moment of extrasolar planets in Sections 4.4 and 4.6 ,

\subsection{Limits of the scaling law concept}

The concept of scaling laws which try to predict the amplitude of the planetary magnetic moment from simple equations using only a few planetary parameters has often been met with scepticism. If one considers the fact that the magnetic field generation by a dynamo is a highly complex, non-linear and chaotic process, (Stevenson 2003), this scepticism is understandable. This section deals with problems and limitations of the scaling law concept.

The validity of magnetic moment scaling laws is usually tested in a double-logarithmic plot of the planetary magnetic moment as a function of some planetary parameter. The first planetary parameter related to the magnetic moment in such a way was the planetary angular momentum. Russell (1978) raises the question whether the fact that the planets lie on a straight line in this diagram really represented a valid test of the physics behind the scaling relation. After all, both variables (magnetic moment and angular momentum) depend on different powers of the planetary radius, so that a certain correlation automatically results.

Using statistical methods, Cain et al.(1995) study the question whether the correlation of planetary magnetic moments and various planetary parameters (e.g. planetary angular momentum, angular momentum of the conducting region and volume of the conducting region) are statistically significant. In this study, Cain et al. (1995) compare the observed correlation between magnetic moment and planetary angular momentum with the scaling which automatically results from geometrical effects. They find that the observed correlation deviates from the expected scaling if only the geometrical effects are considered, but the difference is within the statistical uncertainty. The smallness of the deviation of single data points from the empirical fit, however, can be interpreted as statistically significant. This, however, is true only if one assumes that the observed correlations between

\footnotetext{
${ }^{5}$ The effective temperatures of the planets HD 209458b $\left(T_{\text {eff }}=1130 \pm 150 \mathrm{~K}\right.$, Deming et al. 2005$)$ and TrES-1b $\left(T_{\text {eff }}=1060 \pm 50 \mathrm{~K}\right.$, Charbonneau et al. 2005) have recently been estimated from Spitzer Space Telescope data at $24 \mu \mathrm{m}$, and at $4.5 \mu \mathrm{m}$ and $8.0 \mu \mathrm{m}$, respectively. However, at present it is not possible to separate the contribution of internal heat flux from the strong stellar irradiation. Until more data are available, eqs. 4.30) and 4.31) cannot be used to estimate the planetary magnetic moment of Hot Jupiters.
} 
planetary radius, density, inertial coefficient and rotation period do not have a physical origin. If these correlations were the result of some physical mechanism (for example during the planetary generation), then the observed dependence of the magnetic moment is not statistically significant. In this case, no conclusion about the origin of the magnetic field is possible.

In a similar way, Arge et al. (1995) and Baliunas et al. (1996) examine the connection between angular momentum and magnetic moment of stars. The study of Baliunas et al. (1996), for example, includes 112 main sequence stars. In contrast to the result of Cain et al. (1995) for planets, the studies of Arge et al. (1995) and Baliunas et al. (1996) find a significant correlation for stellar magnetic fields with stellar rotation. The results of Baliunas et al. (1996) show good agreement with the magnetostrophic scaling of Curtis and Ness (1986). Note, however, that the results for main sequence stars given by Arge et al. (1995) and Baliunas et al. (1996) are consistent with a scaling solely based on the geometric effect discussed by Cain et al. (1995).

One should also be aware of the fact that in a double-logarithmic plot points "close" to a certain line can have considerable deviations from points on that line when the numbers are compared. Thus, seemingly small errors can easily reach values of a factor of a few (Cain et al. 1995).

Another problem arising when comparing the results of scaling laws to measured values is the variability of planetary magnetic fields. For the Earth, usually a deviation of a factor of two from the presently observed numbers is attributed to secular variation (Russell 1978, Siscoe 1979). Thus, one must assume the scaling laws to be imperfectly calibrated.

For the reasons presented above, a comparison of different scaling laws is not easily achieved. For all planets of the solar system (except Mercury), Curtis and Ness (1986) find that their magnetostrophic scaling (see Section 4.1.5 for details) yields results very similar to those obtained by the scaling relation of Blackett (1947) described in Section 4.1.1.

Stevenson (1983) explicitely warns against the use of scaling laws. He argues that the planets probably are not similar enough for a single, universal scaling law to fit. It is obvious that a scaling law can only work if all planetary dynamos considered follow the same physical mechanism (Russell 1979). At least, a reasonable scaling law has to fulfil the following requirements (Stevenson 1983):

- The range of validity has to be given. For example, typically used scaling laws do not include the case of no dynamo.

- To any predicted magnetic field strength, it has to be checked whether enough energy is available.

- The connection between toroidal and poloidal magnetic field has to be made clear.

Unfortunately, most of the scaling laws presented in Section 4.1 do not fulfil these requirements. Only one scaling law (4.34) relates toroidal and poloidal magnetic fields; however, it does not consider the available energy. Thus, the condition of sufficient energy has to be checked separately after the application of the scaling law. Another way would be to construct scaling relations based on the available power and not on a force balance. As 
was noted in Section 4.1.9, this approach is problematic for close-in exoplanets which are strongly heated by their host star.

Recently, a numerical approach to derive a magnetic moment scaling relation was taken by Christensen and Aubert (2006). The authors use a considerable set of dynamo simulations, from which an empirical scaling relation is derived. First results seem to indicate that the magnetic field strength is independent of both the electrical conductivity and the rotation rate. However, for numerical reasons, these results were obtained for a parameter range strongly different from planetary values. Therefore, more precise studies are required before any final conclusion can be drawn.

The following limitations apply to the magnetic moment scaling relations presented in Section 4.1.9.

- Magnetic moment scaling relations do not present a precise prediction of the planetary magnetic moment. One should expect the results to be precise only up to a factor of a few (Russell 1979).

- At the same time, scaling laws do not predict the amplitude of magnetic fields generated by a dynamo process, but only present an upper limit. Lower values of the magnetic field are possible, e.g. when not enough energy is available, or if (for some reason) a dynamo is not present.

Within these limitations, magnetic moment scaling relations, whether or not they can be derived from dynamo theory or are solely based on geometrical effects, present a useful tool for the estimation of otherwise unknown and unaccessible quantities (Russell 1978). Only future observations can provide additional insight into the validity of this approach. Until then, scaling laws present the only realistic way to obtain such quantities.

\subsection{Input parameters for gas giants}

While the study of exoplanetary magnetospheres requires the planetary magnetic dipole moment as an important input parameter, no information on exoplanetary magnetic moments is available at present. The existence of a planetary magnetosphere was tentatively deduced from the observation of chromospheric emission from the planet-hosting stars HD 179949 (Shkolnik et al. 2003) and $v$ And (Shkolnik et al. 2005). However, models without a planetary magnetic field are equally able to describe the observations (Preusse 2006), so that presently, still no observational proof for the existence of exomagnetospheres is available (see Section 2.5).

Because of this lack of observational data, the magnetic moment scaling laws discussed in section 4.1 will be applied to estimate the planetary magnetic dipole moment parallel to the rotation axis. For ease of reference, the scaling laws 4.27), (4.32), 4.33) and (4.34) remaining after the discussion of Section 4.1.9 are repeated here:

$$
\begin{array}{rcl}
\mathcal{M} \propto & \rho_{\mathrm{c}}^{1 / 2} \omega r_{\mathrm{c}}^{4} & \\
\mathcal{M} \propto & \rho_{\mathrm{c}}^{1 / 2} \omega^{3 / 4} r_{\mathrm{c}}^{7 / 2} \sigma^{-1 / 4} & \text { (Mizse 1976) } \\
\mathcal{M} \propto & \rho_{\mathrm{c}}^{1 / 2} \omega^{1 / 2} r_{\mathrm{c}}^{3} \sigma^{-1 / 2} & \text { (Mizutani et al. 1992) } \\
\mathcal{M} \propto & \rho_{\mathrm{c}}^{1 / 2} \omega r_{\mathrm{c}}^{7 / 2} & \text { (Sano 1993) }
\end{array}
$$


$\mathcal{M}$ is the planetary magnetic dipole moment, $r_{\mathrm{c}}$ the radius of the dynamo-region and $\omega$ the angular velocity of the planetary rotation. $\rho_{\mathrm{c}}$ and $\sigma$ are the mass density and the electrical conductivity in the dynamo region, respectively.

It is clear from eq. (4.36) to (4.39) that the magnetic moment $\mathcal{M}$ is determined by the internal properties of a planet (i.e. the properties of the dynamo region like $r_{\mathrm{c}}, \rho_{\mathrm{c}}$ ) and not so much by the external parameters which are much easier to measure (e.g. the planetary mass or radius). For this reason, Section 4.3.1 presents a simple model for the interior of gaseous giant planets. In Sections 4.3.2 and 4.3.3 the unknown parameters $r_{\mathrm{c}}$ and $\rho_{\mathrm{c}}$ are deduced from this model. Finally, the rotation rate $\omega$ and the conductivity $\sigma$ will be evaluated in Sections 4.3.4 and 4.3.5.

\subsubsection{The hydrostatic model}

The following derivation of the density profile $\rho(r)$ is based on the work of Chandrasekhar (1957). Within a spherically symmetric gas giant, at any distance $r$ from the centre, the gradient of the gas pressure $p(r)$ is balanced by the gravitational acceleration $g(r)$ :

$$
\frac{d p(r)}{d r}=-g(r) \rho(r)
$$

Here, $\rho(r)$ is the density at the height $r$. Eq. (4.40) is called the equation of hydrostatic equilibrium (Hubbard 1984, Scheffler 1997). Measurements of the gravitational moments of Jupiter and Saturn show that the hydrostatic assumption is valid for both Jupiter and Saturn (Stevenson 1982). Note that for sake of simplicity, the planetary rotation is neglected. Also, the planet is assumed to have no rocky core. Stevenson (1982) and Hubbard and Marley (1989) describe how more complex models can be constructed.

The gravitational acceleration is given by the mass $M_{r}$ contained within the sphere of radius $r$ :

$$
g(r)=\frac{G M_{r}(r)}{r^{2}}
$$

where $G$ denotes the constant of gravity. $M_{r}$ is defined by

$$
M_{r}(r)=\int_{0}^{r} 4 \pi \rho\left(r^{\prime}\right) r^{\prime 2} d r^{\prime} .
$$

Differentiating eq. (4.42) with respect to $r$ leads to

$$
\frac{d M_{r}(r)}{d r}=4 \pi \rho(r) r^{2}
$$

Now eq. (4.41) is inserted into eq. 4.40, which is subsequently multiplied with $r^{2} / \rho$. This results in

$$
\frac{r^{2}}{\rho} \frac{d p(r)}{d r}=-G M_{r}
$$

After differentiation with respect to $r$ and insertion of eq. (4.43), one obtains

$$
\frac{1}{r^{2}} \frac{d}{d r}\left(\frac{r^{2}}{\rho} \frac{d p(r)}{d r}\right)=-4 \pi G \rho(r)
$$


At this point, one has to make an assumption for the equation of state (i.e. the pressuredensity relation). A polytropic relation with

$$
p=K \rho^{1+(1 / \kappa)}
$$

is assumed (Chandrasekhar 1957, Hubbard 1984), where $K$ is the polytropic constant and $\kappa$ is the polytropic index. As will be shown with eq. (4.53) below, $\kappa=1$ is a plausible value for Jupiter. With eq. (4.46) and $\kappa=1$, eq. (4.45) becomes

$$
\frac{1}{r^{2}} \frac{d}{d r}\left(\frac{2 K}{4 \pi G} r^{2} \frac{d \rho(r)}{d r}\right)=-\rho(r) .
$$

Now, the dimensionless variable $\xi$ is introduced with

$$
\begin{aligned}
a & :=\sqrt{\frac{2 K}{4 \pi G}} \\
\xi & :=\frac{r}{a},
\end{aligned}
$$

so that eq. 4.47) can be written in dimensionless form:

$$
\frac{1}{\xi^{2}} \frac{d}{d \xi}\left(\xi^{2} \frac{d \rho(\xi)}{d \xi}\right)=-\rho(\xi)
$$

Equation (4.50) is the Lane-Emden equation of index 1 (Chandrasekhar 1957). The solution is given by (Chandrasekhar 1957, Hubbard 1984)

$$
\rho(\xi)=\rho_{\text {center }} \frac{\sin (\xi)}{\xi},
$$

as can be easily verified by insertion of eq. (4.51) into (4.50). Here, $\rho_{\text {center }}$ is the density at the centre of the planet, i.e. $\rho_{\text {center }}=\rho(r=0)$. With eq. 4.51), the density $\rho$ is monotonically decreasing with increasing value of $\xi$, and it becomes zero for $\xi=\pi$. This defines the radius of the planet.

Coming back to dimensional variables, and introducing the planetary radius $R_{\mathrm{p}}=\pi a$, eq. (4.51) becomes

$$
\rho(r)=\rho_{\text {center }} \frac{\sin \left(\pi \frac{r}{R_{\mathrm{p}}}\right)}{\left(\pi \frac{r}{R_{\mathrm{p}}}\right)} .
$$

Fig. 4.1 shows the dependence of the density $\rho$ on the distance to the planetary centre $r$ according to eq. 4.52).

With eq. (4.48), the planetary radius $R_{\mathrm{p}}$ is given by

$$
R_{\mathrm{p}}=\pi a=\sqrt{\frac{\pi K}{2 G}}
$$

Apparently, for a polytropic index of $\kappa=1$, the radius of a planet does not depend on the central density $\rho_{\text {center }}$ or on the total mass. It is obvious that this regime will not include all gas spheres. For very light gas spheres, the radius should increase with increasing mass, whereas for very heavy gas spheres, the object shrinks when more mass is 


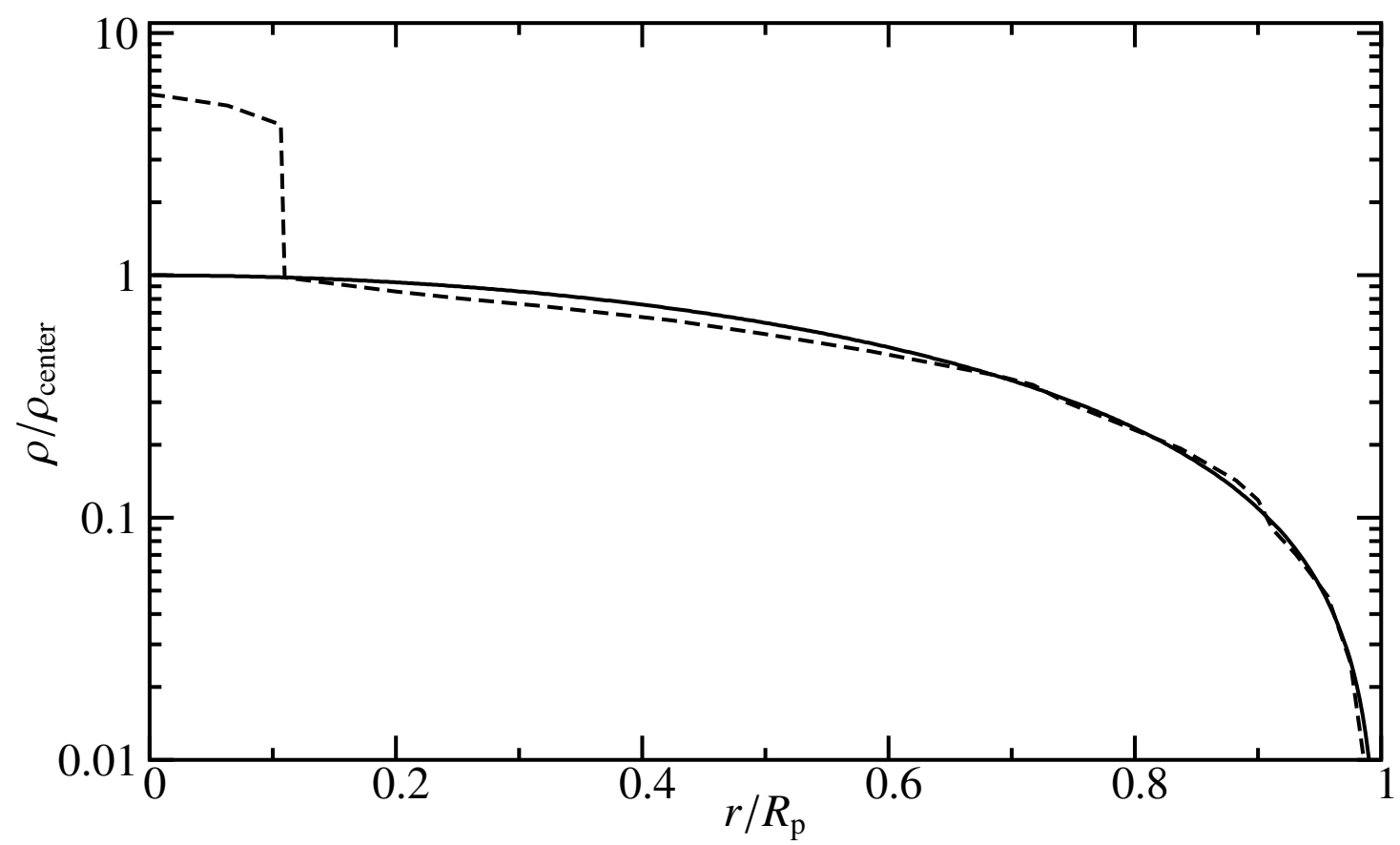

Figure 4.1: Solid line: Internal density within a gas giant according to eq. (4.52). The density, normalised to the central density $\rho_{\text {center }}$, is shown as a function of distance to the planetary centre. The planet is assumed to be in hydrostatic equilibrium, and a polytropic index of $\kappa=1$ was used. Dashed line: The density profile according to the more complex model of Hubbard and Marley (1989. Figure 10), normalised to the density just outside the inner core. See text for discussion.

added. This is the case for white dwarfs ${ }^{6}$ (Hubbard 1984). Thus, the polytropic index $\kappa$ is a function of the mass of the object, and the maximum possible radius is given by the $\kappa=1$ polytrope. In other words, the polytropic index should be close to 1 near the maximum allowed radius. By examining the theoretical mass versus radius relation, Hubbard (1984) finds the maximum radius for a sphere of pure hydrogen to be $R_{\mathrm{p} \text {, } \max }=82600$ $\mathrm{km}$. For heavier elements, the value for $R_{\mathrm{p}, \max }$ is always lower than for hydrogen. For example, $R_{\mathrm{p}, \max }=35000 \mathrm{~km}$ for helium (Hubbard 1984 ), and even less for all other elements. The observed radii of Jupiter, Saturn and of the known transiting extrasolar planets HD 209458b, OGLE-TR-56b, OGLE-TR-111b, OGLE-TR-113b, OGLE-TR-132b and TrES-1 are much larger than the limit for a sphere consisting of helium. For this reason, it immediately follows that these planets are predominantly composed of hydrogen? 7 . Additionally, the radius of Jupiter $R_{\mathrm{J}}=71492 \mathrm{~km}$ is close to the maximum allowed value, so that a polytropic index close to one can be assumed for Jupiter and Jupiter-sized extrasolar planets. For Jupiter, the validity of this assumption is also confirmed by the measurements of the gravitational moments (Stevenson 1982, Hubbard 1984). For Sat-

\footnotetext{
${ }^{6}$ It has to be kept in mind that eq. $\sqrt{4.40}$ does not include radiation pressure or heating by nuclear processes. For this reason, stars cannot be described within this framework.

${ }^{7}$ It is interesting to note that low-mass stars close to the hydrogen burning limit can have radii similar to those of giant gaseous planets (Pont et al.2005). For this reason, in addition to the radius of the object, the mass has to be determined to ensure that the object is a planet and not a star. For the transiting exoplanets discussed here, the masses were found to be close to that of Jupiter, so that they can safely be assumed to be giant planets rather than stars.
} 
urn, the gravitational moments do not confirm the approximation $\kappa=1$. This is explained by the presence of a massive central core.

With the density profile given by eq. (4.52), one can calculate the average density $\bar{\rho}$ and compare its value to the central density $\rho_{\text {center }}$ :

$$
\bar{\rho}=\frac{\int_{0}^{R_{\mathrm{p}}} 4 \pi \rho(r) r^{2} d r}{\frac{4 \pi}{3} R_{\mathrm{p}}^{3}} .
$$

After insertion of eq. (4.52) and integration, one obtains

$$
\bar{\rho}=\frac{3}{\pi^{2}} \rho_{\text {center }} \approx 0.30 \rho_{\text {center }},
$$

which is also the result obtained by Hubbard (1984).

From eq. (4.55) it can be seen that the average density $\bar{\rho}$ of the planet is proportional to the central density $\rho_{\text {center }}$. Because the average density of a planet can be inferred from its observed mass $M_{\mathrm{p}}$ and radius $R_{\mathrm{p}}$, it is helpful to replace $\rho_{\text {center }}$ in the density profile (4.52):

$$
\rho(r)=\left(\frac{\pi M_{\mathrm{p}}}{4 R_{\mathrm{p}}^{3}}\right) \frac{\sin \left(\pi \frac{r}{R_{\mathrm{p}}}\right)}{\left(\pi \frac{r}{R_{\mathrm{p}}}\right)} .
$$

This result will be used in the following sections.

Eq. (4.52) and (4.56) only present a rough estimation for the density profile within a giant planet. For example, the phase transition between molecular and metallic hydrogen was not taken into account. For this reason, it is important to compare the results obtained in this section with more realistic calculations. Figure 4.1 compares the density profile obtained from eq. (4.56) with that of the more complex model of Hubbard and Marley (1989). It can be seen that, except for the rocky core, which is not included in the current estimation, both models agree remarkably well. For this reason, this model is a valid approximation for the estimation of $r_{\mathrm{c}}$ and $\rho_{\mathrm{c}}$.

\subsubsection{Size of the dynamo region $r_{\mathrm{c}}$}

One of the most important unknown parameters in eq. (4.36) to (4.39) is the size of the dynamo region $r_{\mathrm{c}}$. It has a large exponent, but even in the solar system, it is not easily accessible to measurements. To overcome this problem, Curtis and Ness (1986) use an empirical scaling law to determine the core radius $r_{\mathrm{c}}$ as a function of the planetary mass $M_{\mathrm{p}}$. The best fit to our solar system (using the planets Mercury, Earth, Jupiter and Saturn) was found to be given by:

$$
r_{\mathrm{c}} \propto M_{\mathrm{p}}^{0.44}
$$

where $M_{\mathrm{p}}$ is the mass of the planet.

In this approach the core radius only depends on the mass of the planet. This seems reasonable for planets which have evolved to some equilibrium state, i.e. for which the radius $R_{\mathrm{p}}$ is well determined by the mass $M_{\mathrm{p}}$. For young planets, where for a given mass 
the radius is a function of the age (Guillot et al. 1996, Burrows et al. 1997, 2000, Guillot and Showman 2002, Burrows et al. 2003, 2004a), it is better to consider both the mass $M_{\mathrm{p}}$ and the radius $R_{\mathrm{p}}$ in a scaling law of the form (Grießmeier et al. 2004)

$$
r_{\mathrm{c}} \propto M_{\mathrm{p}}^{\alpha} R_{\mathrm{p}}^{\beta}
$$

This situation is also typical for "Hot Jupiters", where strong stellar irradiation is supposed to delay the planetary contraction (Burrows et al. 2000, 2003, 2004a), thus leading to an increased planetary radius. At first, the measured radius of HD 209458b (Henry et al. 2000, Charbonneau et al. 2000, Mazeh et al. 2000, Jha et al. 2000) seemed to be too large even for a strongly irradiated gas giant. Different explanations were suggested, like the downward transport of kinetic energy generated by the intense stellar irradiation (Guillot and Showman 2002, Showman and Guillot 2002, Bodenheimer et al. 2003), viscous dissipation heating by shear flows in the atmosphere (Burkert et al. 2005), the dissipation of energy arising from tidal circularisation ${ }^{8}$ (Bodenheimer et al. 2003), or tidal heating by gradual coplanarisation (Sato et al.2005). Other works, however, suggest that an additional source of energy may not be required. Because the observed "transit radius" is not identical to the standard 1 bar radius, and considering the observational errors, the measured radius of HD 209458b is just consistent with a model excluding additional heat sources (Burrows et al.2003). Similarly, for the planet OGLE-TR-56b, no additional heat source is required (Burrows et al. 2004a). It is found that the stellar irradiation increases the planetary radius by approximately $0.2-0.3 R_{\mathrm{J}}$ (Burrows et al. 2003, 2004a). For this reason, it seems appropriate to include the planetary radius in the estimation of $r_{\mathrm{c}}$ and use eq. 4.58).

Normalising $r_{\mathrm{c}}, M_{\mathrm{p}}$ and $R_{\mathrm{p}}$ to the respective values of Jupiter and using the best fit for the planets Saturn, Uranus and Neptune to eq. (4.58), one obtains

$$
r_{\mathrm{c}} \propto M_{\mathrm{p}}^{0.75} R_{\mathrm{p}}^{-0.96}
$$

For this fit, the following values were adopted: $r_{\mathrm{c}}=0.9 R_{\mathrm{p}}$ for Jupiter (Nellis 2000), $r_{\mathrm{c}}=0.5 R_{\mathrm{p}}$ for Saturn (Nellis 2000), $r_{\mathrm{c}}=18000 \mathrm{~km}$ (i.e. $r_{\mathrm{c}}=0.7 R_{\mathrm{p}}$ ) for Uranus (Hubbard 1984), and $r_{\mathrm{c}}=19000 \mathrm{~km}$ (i.e. $r_{\mathrm{c}}=0.75 R_{\mathrm{p}}$ ) for Neptune (Hubbard 1984). Note that for Jupiter and Saturn, the transition between metallic and molecular phase probably is continuous (Guillot|1999, Nellis 2000). For this reason, the values given above correspond to the size of the highly conducting region responsible for the magnetic field generation. The values were obtained by laboratory experiments (Nellis 2000). Because of the gradual change in conductivity, this region is larger than the region of the pure metallic phase (Smoluchowski 1975, Stevenson|1982).

Relation (4.59) yields a more realistic behaviour than eq. (4.57). On the one hand, if the radius is kept constant, an increase in mass will lead to a larger core. On the other hand, keeping the mass constant and increasing the radius will decrease the average density, and the volume of high enough density for the dynamo will also decrease. Similarly, for a fixed average density (i.e. $R_{\mathrm{p}} \propto M_{\mathrm{p}}^{1 / 3}$ ), increasing the mass increases the core radius.

\footnotetext{
${ }^{8}$ Recent infrared observations of the secondary eclipse of HD 209458 by be Spitzer Space Telescope indicate that a dynamically significant eccentricity is unlikely. Therefore, it seems improbable that this effect is relevant for HD 209458b.
} 


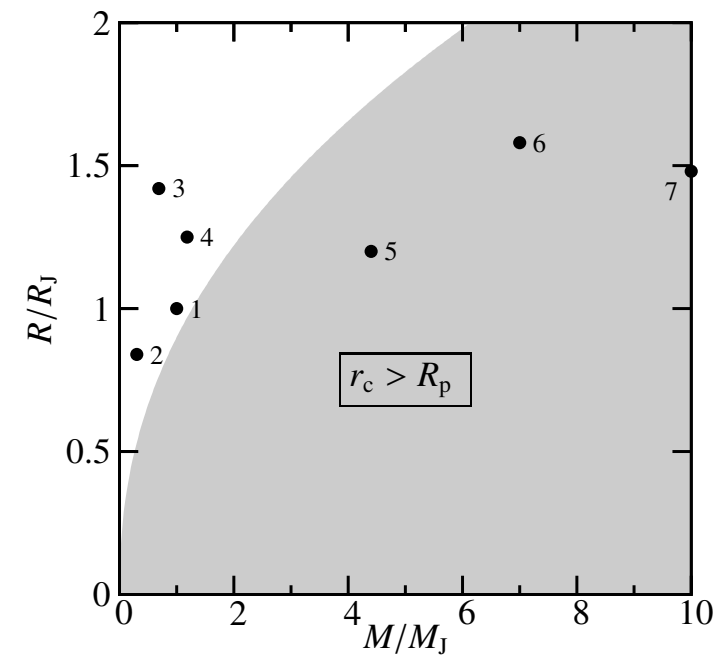

(a) Test of the condition $r_{\mathrm{c}} \leq R_{\mathrm{p}}$ for eq. 4.57p.

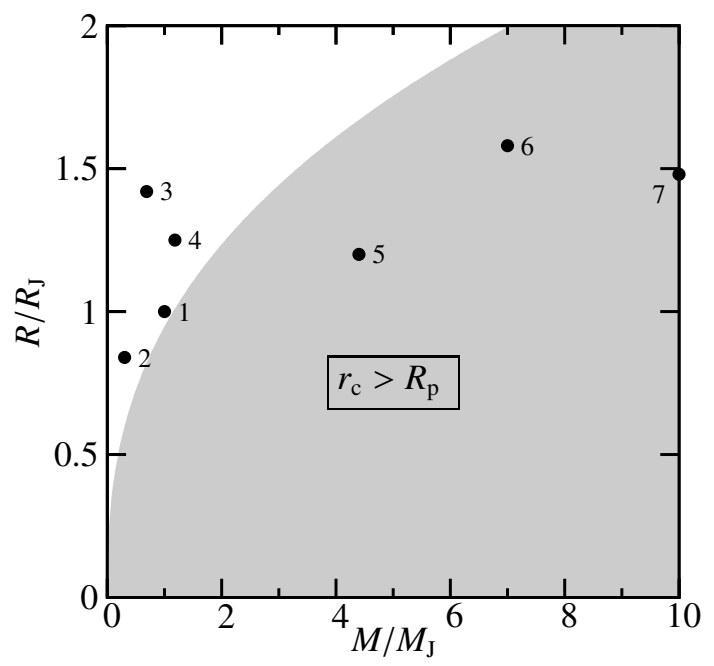

(b) Test of the condition $r_{\mathrm{c}} \leq R_{\mathrm{p}}$ for eq. 4.59p.

Figure 4.2: Test of the condition $r_{\mathrm{c}} \leq R_{\mathrm{p}}$ in the $M_{\mathrm{p}}-R_{\mathrm{p}}$ parameter space for eq. (4.57) in Figure 4.2(a), and for eq. (4.59) in Figure 4.2(b), The shaded area denotes regions within the parameter space where the condition is not satisfied, and the scaling law for $r_{\mathrm{c}}$ cannot be applied. Also shown as numbered black dots: The planets Jupiter (1), Saturn (2), HD 209458b (3), OGLE-TR 56b (4) and $\tau$ Bootes (5,6,7). For $\tau$ Bootes, three different models are studied, see Section 2.6.2.

Of course, relations 4.57) and 4.59) only have a physical meaning if the condition

$$
r_{\mathrm{c}} \leq R_{\mathrm{p}}
$$

is fulfilled 9 Fig. 4.2 explores for which values of $R_{\mathrm{p}}$ and $M_{\mathrm{p}}$ this condition is satisfied. One can clearly see that this is not the case for all the planets of interest. Especially the large and heavy planets (numbers 5, 6 and 7 in the figure) which are interesting for the study of nonthermal radio emission (see Section 6) are affected. In these cases, the core radius $r_{\mathrm{c}}$ is set equal to the planetary radius $R_{\mathrm{p}}$ for all subsequent calculations (i.e. $r_{\mathrm{c}}=$ $R_{\mathrm{p}}$ ). This approach may seem arbitrary, but it is reasonable to assume a monotonous increase in $r_{\mathrm{c}} / R_{\mathrm{p}}$ with planetary mass $M_{\mathrm{p}}$. Thus, the real value for $r_{\mathrm{c}}$ will fall into the range $0.9 \leq r_{\mathrm{c}} / R_{\mathrm{p}} \leq 1.0$, and the error made by assuming $r_{\mathrm{c}}=R_{\mathrm{p}}$ is tolerable.

Sánchez-Lavega (2004) introduces a different way of estimating the size of the dynamo region $r_{\mathrm{c}}$. Assuming that the density at which the transition from the molecular phase to the liquid metallic phase ${ }^{10}$ takes place is given by

$$
\rho=\rho_{\text {transition }}=1.0 \cdot 10^{3} \mathrm{~kg} / \mathrm{m}^{3},
$$

the density distribution $\rho(r)$ described by eq. 4.56 can be inverted to find the distance for which $\rho(r)=\rho_{\text {transition. }}$. The resulting distance is then identified with the radius of

\footnotetext{
${ }^{9}$ Several publications exist where this condition was not evaluated, thus overestimating the size of the dynamo region $r_{\mathrm{c}}$ and the magnetic moment $\mathcal{M}$ (Farrell et al. 1999, Lazio et al. 2004, Farrell et al. 2004).

${ }^{10}$ A continuous transition is more likely, but in order to obtain simple estimations of the magnetic field, a noncontinuous model is preferred.
} 
the dynamo region $r_{\mathrm{c}}$. This estimation can be used for planets in the mass range $0.3 \leq$ $M_{\mathrm{p}} / M_{\mathrm{J}} \leq 10$, i.e. for planets with masses between that of Saturn and ten times the mass of Jupiter (obtained from Sánchez-Lavega 2004). Because the transition is expected to take place in the density range of $7.0 \cdot 10^{2} \mathrm{~kg} / \mathrm{m}^{3} \leq \rho_{\text {transition }} \leq 1.6 \cdot 10^{3} \mathrm{~kg} / \mathrm{m}^{3}$ (obtained from Sánchez-Lavega 2004), an upper limit for the magnetic moment is obtained by calculating $r_{\mathrm{c}}$ with

$$
\rho=\rho_{\text {transition }}=7.0 \cdot 10^{2} \mathrm{~kg} / \mathrm{m}^{3}
$$

instead of eq. (4.61). This approach has the advantage that condition (4.60) is automatically satisfied. However, for planets with low enough mass or large enough radius, another limitation comes into play. When the mean density of the planet is low enough, the central density cannot reach the value of $\rho_{\text {transition }}$ required by eq. (4.61) or eq. (4.62). In that case, the transition to liquid metallic hydrogen does not occur for the given planet. As this approach is based on the existence of liquid metallic hydrogen, it yields $r_{\mathrm{c}}=0$ and thus $\mathcal{M}=0$ for such planets. Figure 4.3 shows for which values of $M_{\mathrm{p}}$ and $R_{\mathrm{p}}$ this would be the case for the two different values of $\rho_{\text {transition }}$ given by eq. (4.61) and eq. (4.62), respectively.

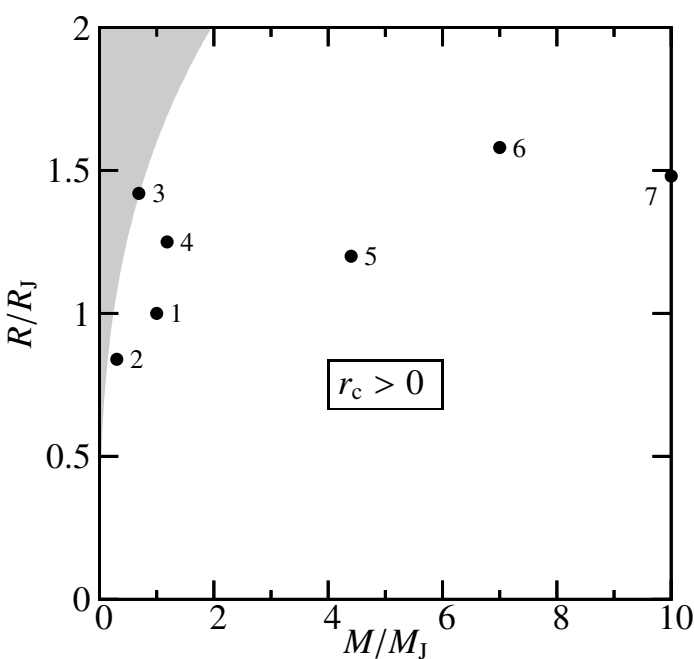

(a) Test of the condition $r_{\mathrm{c}}>0$ for eq. 4.61.

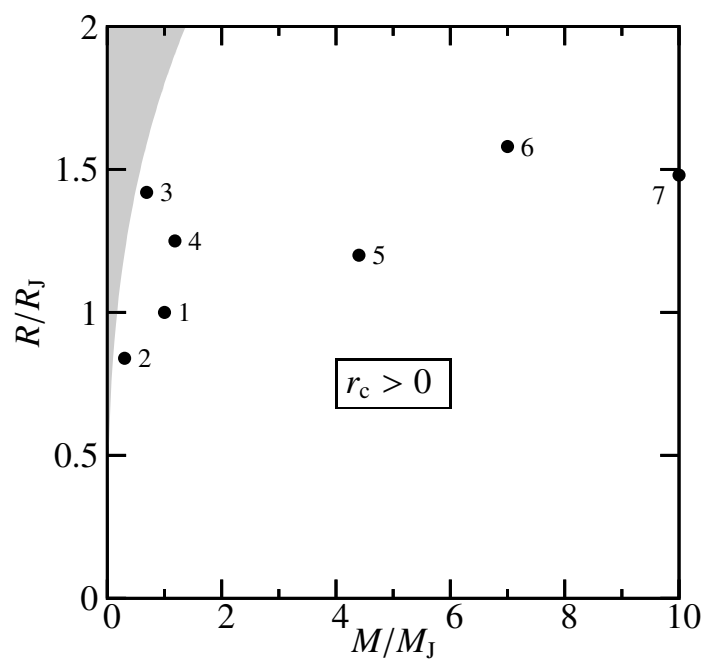

(b) Test of the condition $r_{\mathrm{c}}>0$ for eq. 4.62,

Figure 4.3: Shaded area: Region of the parameter space in which planets do not have liquid metallic hydrogen cores. Number dots: Jupiter (1), Saturn (2), HD 209458b (3), OGLE-TR 56b (4) and different models for $\tau$ Bootes $(5,6,7)$. Figure 4.3(a): $\rho_{\text {transition }}=1000 \mathrm{~kg} / \mathrm{m}^{3}$. For this value of $\rho_{\text {transition, }}$, the planet HD 209458b does not have a liquid metallic hydrogen core. Figure 4.3(b); $\rho_{\text {transition }}=700 \mathrm{~kg} / \mathrm{m}^{3}$. In this case, all of the studied planets have a liquid metallic hydrogen core.

Finally, the sizes of the dynamo region $r_{\mathrm{c}}$ resulting from the different models presented in this section are compared. Both eq. (4.57) (Curtis and Ness 1986) and eq. (4.59) (Grießmeier et al.2004) result from an empirical fit to the solar system, whereas eq. (4.56) (Sánchez-Lavega 2004) with either $\rho_{\text {transition }}=1000 \mathrm{~kg} / \mathrm{m}^{3}$ or $\rho_{\text {transition }}=700 \mathrm{~kg} / \mathrm{m}^{3}$ was derived from the hydrostatic model presented in Section 4.3.1. While the simple model of 
Curtis and Ness (1986) has the disadvantage of not including the planetary radius $R_{\mathrm{p}}$, it can directly be applied to a larger number of exoplanets ${ }^{11}$. The different values obtained are shown in Table 4.1. Both the limitation of the empirical models for large planets (when one has to set $r_{\mathrm{c}}=1.0$ ) and the limitation of the hydrostatic model for planets of low density (when $r_{\mathrm{c}}=0$ ) can be seen (see also Fig. 4.2 and Fig. 4.3). Apart from that, the precise value of $r_{\mathrm{c}}$ does not strongly depend on the precise value of $\rho_{\text {transition }}$ (cf. Models 3 and 4).

\begin{tabular}{|l||c|c||c|c|c|c|}
\hline \multicolumn{1}{|c||}{ Planet } & \multirow{2}{*}{$R_{\mathrm{p}}\left[R_{\mathrm{J}}\right]$} & \multirow{2}{*}{$M_{\mathrm{p}}\left[M_{\mathrm{J}}\right]$} & \multicolumn{4}{c|}{$r_{\mathrm{c}} / R_{\mathrm{p}}$} \\
\cline { 5 - 7 } & & & Model 1 & Model 2 & Model 3 & Model 4 \\
\hline \hline Jupiter & 1.0 & 1.0 & 0.9 & 0.9 & 0.79 & 0.85 \\
Saturn & 0.84 & 0.3 & 0.63 & 0.51 & 0.62 & 0.72 \\
HD 209458b & 1.42 & 0.69 & 0.54 & 0.34 & 0.0 & 0.44 \\
OGLE-TR-56b & 1.23 & 1.45 & 0.77 & 0.66 & 0.67 & 0.76 \\
$\tau$ Bootes (light) & 1.2 & 4.4 & 1.0 & 1.0 & 0.91 & 0.94 \\
$\tau$ Bootes (medium) & 1.58 & 7.0 & 1.0 & 1.0 & 0.88 & 0.91 \\
$\tau$ Bootes (heavy) & 1.48 & 10.0 & 1.0 & 1.0 & 0.93 & 0.95 \\
\hline
\end{tabular}

Table 4.1: Relative size of the dynamo region according to different models. Model 1: eq. (4.57) according to Curtis and Ness (1986). Model 2: eq. (4.59) according to Grießmeier et al. (2004). Model 3: eq. 4.56) and eq. (4.61) according to Sánchez-Lavega (2004). Model 4: eq. (4.56) and eq. (4.62). See text for details.

None of the presented models includes the effect of strong stellar irradiation. For "Hot Jupiters", the high temperatures may lead to additional ionisation, thus slightly increasing the core radius $r_{\mathrm{c}}$ and the magnetic moment $\mathcal{M}$. Also, Hot Jupiters probably have a larger radius $R_{\mathrm{p}}$ than comparable planets without strong stellar irradiation. Depending on whether the increased radius of extrasolar planets affects the structure of the whole planet or only alters the outer layers, the equations of hydrostatic equilibrium with an $\kappa=1$ polytrope may not be a good approximation for the outer layers, or even for the planet as a whole. More detailed models for insulated extrasolar giant planets can be found, for example, in Seager and Sasselov (1998), Goukenleuque et al. (2000), Guillot and Showman (2002), Showman and Guillot (2002), and in Burrows et al. (2003).

Because a theory-based model should be preferred to an empirical scaling, the hydrostatic Model 4 will be employed in the following. To obtain an upper limit for the size of the dynamo region $r_{\mathrm{c}}$ and thus for the planetary magnetic moment, eq. (4.62) will be used for the critical density where the transition to the metallic state occurs (i.e. Model 4 in Table 4.1). The value of $r_{\mathrm{c}}$ obtained from equation (4.62) will also be used for the calculation of the average density within the dynamo region (Section 4.3.3). In the cases where no information on the planetary radius is available, eq. (4.57) together with (4.60)

\footnotetext{
${ }^{11}$ For all known extrasolar planets, at least a lower limit for the mass is known, while the radius could be determined only for very few planets (see Section 2.6.1).
} 
can be used.

\subsubsection{Density of the dynamo region $\rho_{\mathrm{c}}$}

The second parameter that is required for the estimation of a planetary magnetic dipole moment is the density within the dynamo region $\rho_{\mathrm{c}}$. Hubbard (1984) shows that for a planet like Jupiter the mean density of the planet $\bar{\rho}=3 M_{\mathrm{p}} /\left(4 \pi R_{\mathrm{p}}^{3}\right)$ can be assumed to be proportional to the central density $\rho_{\text {center }}$ (i.e. the density at the centre of the planet). For the estimation of planetary magnetic moments, it is sometimes assumed that a similar proportionality also holds for the core density $\rho_{\mathrm{c}}$, i.e. the density which is found by averaging from the centre to $r_{\mathrm{c}}$ (Curtis and Ness 1986, Grießmeier et al. 2004):

$$
\rho_{\mathrm{c}} \propto \bar{\rho}=\frac{3 M_{\mathrm{p}}}{4 \pi R_{\mathrm{p}}^{3}} .
$$

In this section, it will be shown that such a proportionality is valid only approximately; for a better estimation of the core density, the size of the core $r_{\mathrm{c}}$ enters as an additional parameter. While the more simplified estimation (4.63) can be applied for planets of the size of Saturn and larger, it should be avoided for smaller gas giants (see below).

In Section 4.3.1 the density variation $\rho(r)$ within the planet was determined. From eq. (4.56) one can calculate the average density within a certain volume and compare its value to the central density $\rho_{\text {center }}$ or to the mean density of the planet $\bar{\rho}$. In contrast to Section 4.3.1, the volume over which the average is taken will be kept variable. For this reason, $\bar{\rho}_{\lambda}$ is defined as the average density in the sphere between $0 \leq r \leq \lambda R_{\mathrm{p}}$ (i.e. $\lambda$ is the fractional radius over which the average is taken):

$$
\bar{\rho}_{\lambda}=\frac{\int_{0}^{\lambda R_{\mathrm{p}}} 4 \pi \rho(r) r^{2} d r}{\frac{4 \pi}{3}\left(\lambda R_{\mathrm{p}}\right)^{3}} .
$$

Inserting eq. 4.56 and integrating, one obtains

$$
\begin{aligned}
\bar{\rho}_{\lambda} & =\frac{3 M_{\mathrm{p}}}{4 \lambda^{3} R_{\mathrm{p}}^{5}}\left[-\frac{R_{\mathrm{p}} r}{\pi} \cos \left(\pi \frac{r}{R_{\mathrm{p}}}\right)+\frac{R_{\mathrm{p}}^{2}}{\pi^{2}} \sin \left(\pi \frac{r}{R_{\mathrm{p}}}\right)\right]_{r=0}^{r=\lambda R_{\mathrm{p}}} \\
& =\frac{3 M_{\mathrm{p}}}{4 \pi \lambda^{3} R_{\mathrm{p}}^{3}}\left[\frac{\sin (\lambda \pi)}{\pi}-\lambda \cos (\lambda \pi)\right] .
\end{aligned}
$$

If the averaging is performed over the whole planet, i.e. $\lambda=1$, one obtains

$$
\bar{\rho}=\bar{\rho}_{\lambda=1}=\frac{3 M_{\mathrm{p}}}{4 \pi R_{\mathrm{p}}^{3}},
$$

as expected. Thus, eq. 4.65) can be simplified to

$$
\bar{\rho}_{\lambda}=\frac{\bar{\rho}}{\lambda^{3}}\left[\frac{\sin (\lambda \pi)}{\pi}-\lambda \cos (\lambda \pi)\right] .
$$

For the magnetic moment scaling law, the core density $\rho_{\mathrm{c}}$ is required. If $\lambda$ is taken to be $r_{\mathrm{c}} / R_{\mathrm{p}}$, where the size of the core $r_{\mathrm{c}}$ was discussed in detail in Section 4.3.2, then 


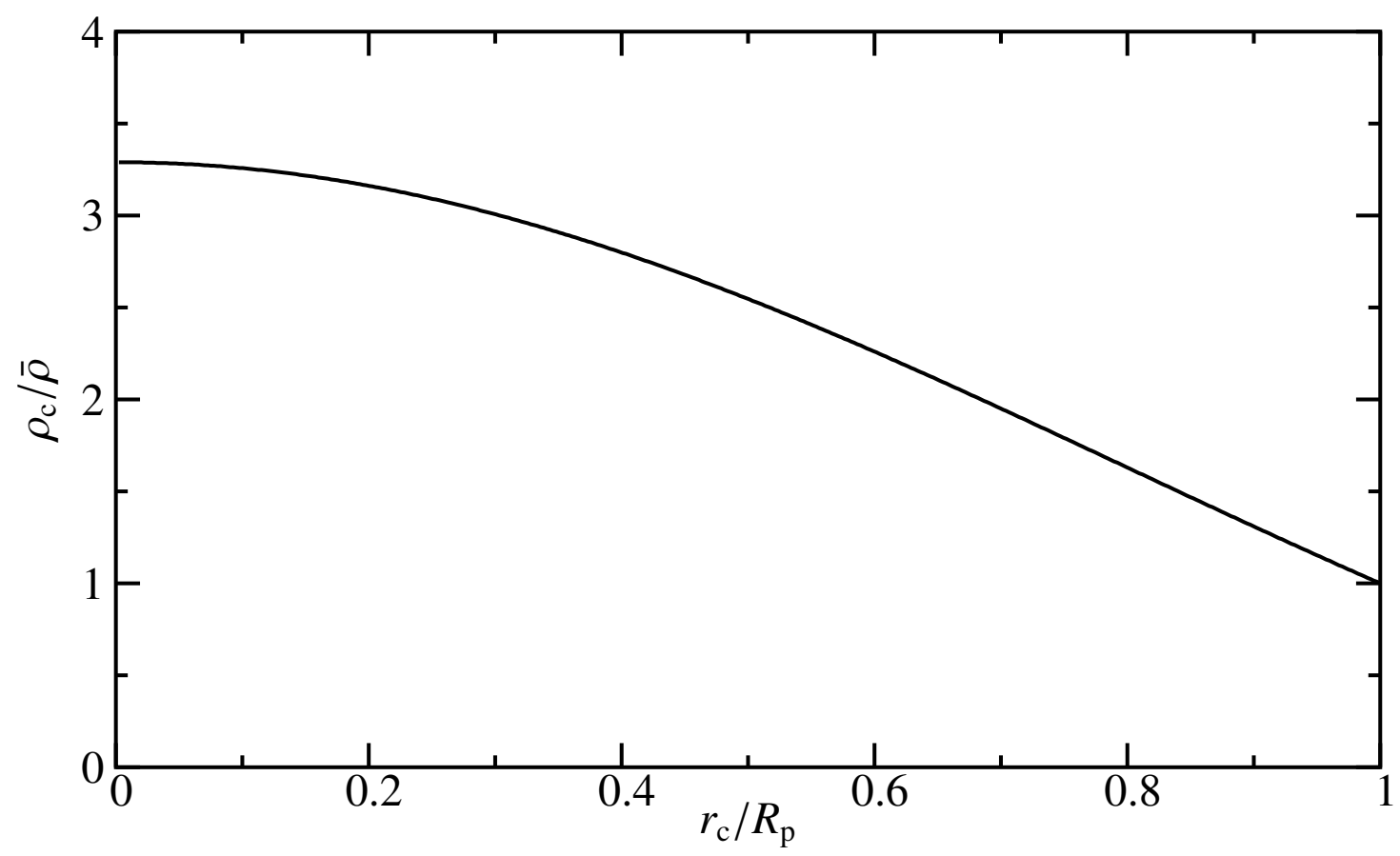

Figure 4.4: Average density of the planetary core (relative to the average density of the planet) as a function of the fractional size of the core $r_{\mathrm{c}} / R_{\mathrm{p}}$ according to eq. 4.68).

eq. (4.67) can be used to obtain $\rho_{\mathrm{c}}$ (i.e. $\bar{\rho}_{\lambda=\left(r_{\mathrm{c}} / R_{\mathrm{p}}\right)}=\rho_{\mathrm{c}}$ ). For transiting planets, the average density $\bar{\rho}=3 M_{\mathrm{p}} /\left(4 \pi R_{\mathrm{p}}^{3}\right)$ can be inferred from observations. Thus, the core density is given by

$$
\rho_{\mathrm{c}}=\bar{\rho}\left(\frac{R_{\mathrm{p}}^{3}}{r_{\mathrm{c}}^{3}}\right)\left[\frac{1}{\pi} \sin \left(\frac{r_{\mathrm{c}}}{R_{\mathrm{p}}} \pi\right)-\frac{r_{\mathrm{c}}}{R_{\mathrm{p}}} \cos \left(\frac{r_{\mathrm{c}}}{R_{\mathrm{p}}} \pi\right)\right] .
$$

The relation between $\rho_{\mathrm{c}}$ and $\bar{\rho}$ as a function of relative core size $r_{\mathrm{c}} / R_{\mathrm{p}}$ is depicted in Fig. 4.4.

In Table 4.2 the core densities resulting from the different models are compared. "Model 0" corresponds to the simplified approximation $\rho_{\mathrm{c}} \propto \bar{\rho}$. In all other models the core density depends on the core size as given by eq. (4.68). The models differ only in the approaches chosen for the size of the core $r_{\mathrm{c}}$, which are taken from Section 4.3.2. Note that in one case (Model 3), for HD 209458b the density in the planet is not high enough to allow a transition to the metallic phase. No magnetic field will be generated in this case. Apart from that, the precise value of $\rho_{\text {transition }}$ does not have a strong influence on the result (cf. Models 3 and 4). For the estimation of the planetary magnetic dipole moments (Section 4.4), the values of Model 4 will be used, as discussed above.

With eq. 4.55), it was already shown that the average density $\bar{\rho}$ of the planet is proportional to the central density $\rho_{\text {center. Eq. }}$ (4.68) shows that the same is true for the average core density $\bar{\rho}_{\lambda}$ of the planet only if the relative size of the core $r_{\mathrm{c}} / R_{\mathrm{p}}$ is kept fixed. However, in Section 4.3.2 it was shown that $r_{\mathrm{c}} / R_{\mathrm{p}}$ is a function of the planetary mass $M_{\mathrm{p}}$ and radius $R_{\mathrm{p}}$. If this dependence of the density $\rho_{\mathrm{c}}$ on the core size $r_{\mathrm{c}}$ is not taken into account (i.e. if in the evaluation of $\rho_{\mathrm{c}}$ the parameter $\lambda$ is assumed to be the same for all planets), an additional error is introduced. Especially for planets smaller then Saturn 
4.3 Input parameters for gas giants

\begin{tabular}{|l||c|c||c|c|c|c|c|}
\hline \multirow{2}{*}{ Planet } & $R_{\mathrm{p}}$ & $M_{\mathrm{p}}$ & \multicolumn{5}{|c|}{$\rho_{\mathrm{c}} / \rho_{\mathrm{c}, \mathrm{J}}$} \\
\cline { 5 - 8 } & {$\left[R_{\mathrm{J}}\right]$} & {$\left[M_{\mathrm{J}}\right]$} & Model 0 & Model 1 & Model 2 & Model 3 & Model 4 \\
\hline \hline Jupiter & 1.0 & 1.0 & 1.0 & 1.0 & 1.0 & 1.0 & 1.0 \\
Saturn & 0.84 & 0.3 & 0.51 & 0.84 & 0.97 & 0.68 & 0.65 \\
HD 209458b & 1.42 & 0.69 & 0.24 & 0.45 & 0.54 & - & 0.44 \\
OGLE-TR-56b & 1.23 & 1.45 & 0.78 & 0.79 & 0.96 & 0.74 & 0.72 \\
$\tau$ Bootes (light) & 1.2 & 4.4 & 2.55 & 1.95 & 1.95 & 1.96 & 2.07 \\
$\tau$ Bootes (med.) & 1.58 & 7.0 & 1.77 & 1.36 & 1.36 & 1.48 & 1.54 \\
$\tau$ Bootes (heavy) & 1.48 & 10.0 & 3.08 & 2.36 & 2.36 & 2.29 & 2.43 \\
\hline
\end{tabular}

Table 4.2: Relative density in the dynamo region according to different models. Model 0: eq. (4.63). Model 1: eq. (4.68) combined with eq. 4.57) from Curtis and Ness (1986). Model 2: eq. (4.68) combined with eq. (4.59) from Grießmeier et al. (2004). Model 3: eq. (4.68) combined with eq. (4.56) and eq. (4.61) from Sánchez-Lavega (2004). Model 4: eq. (4.68) combined with eq. 4.56) and eq. (4.62). See text for details.

$\left(M_{\mathrm{p}}<0.3 M_{\mathrm{J}}\right)$, large errors are expected because in this case, the location $r_{\mathrm{c}} / R_{\mathrm{p}}$ where the transition density $\rho_{\text {transition }}$ is reached strongly depends on the average density and thus on the planetary mass and radius. This can be seen when Fig. 4.1 is inverted, or from the relative difference between Model 3 and Model 4 for various planetary masses in Table 4.1. With $r_{\mathrm{c}} / R_{\mathrm{p}}$ strongly depending on the planetary parameters for light planets, the factor between $\rho_{\mathrm{c}}$ and $\bar{\rho}$ will also be variable, see eq. (4.68). For larger planets, however, the differences are much smaller, and the error introduced does not exceed a factor of 2 (compare Model 0 and Model 4 in Table 4.2). For this reason, the more simplified approach $\rho_{\mathrm{c}} \propto \bar{\rho}$ of eq. (4.63) (Curtis and Ness 1986, Grießmeier et al. 2004) can be used as a first approximation. For a better estimation, however, the additional correction introduced by eq. (4.68) will be taken into account, using $r_{\mathrm{c}}$ as obtained from eq. 4.62) in Section 4.3.2.

\subsubsection{Planetary rotation rate $\omega$}

Another important parameter required for the evaluation of the magnetic moment scaling laws (4.36) to 4.39) is the planetary rotation rate $\omega$. Depending on the orbital distance of the planet and the timescale for synchronous rotation (as presented in Section 3.1.1), three cases are discussed.

1. For planets at small enough distances for which the timescale for tidal locking is small (i.e. $\tau_{\text {sync }} \leq 100 \mathrm{Myr}$ ), the rotation period is taken to be synchronised with the orbital period ( $\left.\omega=\omega_{\mathrm{f}} \approx \omega_{\text {orbit }}\right)$. For gas giants, the orbital frequency $\omega_{\text {orbit }}$ is known very accurately from measurements (see Section 2.6.2). As was already mentioned in Section 3.1.2 perfect tidal locking should not be expected. Strong stellar heating creates thermal atmospheric tides, leading to a deviation from synchronous rotation (Showman and Guillot 2002, Laskar and Correia 2004). According to the rsults of 
Showman and Guillot (2002), the corresponding error for $\omega$ could be as large as a factor of two. For this reason, both the cases $\omega_{\mathrm{f}}=\omega_{\text {orbit }}$ and $\omega_{\mathrm{f}}=2 \omega_{\text {orbit }}$ will be considered.

2. Planets with distances resulting in $100 \mathrm{Myr} \leq \tau_{\text {sync }} \leq 10$ Gyr may or may not be subject to tidal locking. This will, for example, depend on the exact age of the planetary system, which is typically in the order of a few Gyr. For this reason, the upper limit for the magnetic moment of such a planet is calculated without tidal locking (i.e. $\omega=\omega_{\mathrm{J}}$, see below), and the lower limit for the magnetic moment is calculated with tidal locking (i.e. $\omega=\omega_{\mathrm{f}}$, see above).

3. For planets far away from the central star, the timescale given by eq. (3.7) is very large. For planets with $\tau_{\text {sync }} \geq 10 \mathrm{Gyr}$, the effect of tidal interaction can be neglected, and the planetary rotation rate can be assumed to be equal to the initial rotation rate $\omega_{\mathrm{i}}$. Unfortunately, $\omega_{\mathrm{i}}$ is not well constrained by planetary formation theories, but a primordial rotation period of the order of 10 hours is consistent with solar system data (Hubbard 1984, Chapter 4). In the following, the initial rotation rate will be assumed to be equal to the current rotation rate of Jupiter (i.e. $\omega=\omega_{\mathrm{i}}=\omega_{\mathrm{J}}$ ).

Typically, this results in smaller rotation rates for tidally locked planets than for freely rotating planets. The implications this has for the estimated magnetic moments of tidally locked Hot Jupiters will be discussed in Section 4.4 .

\subsubsection{Conductivity within the dynamo region $\sigma$}

Finally, the conductivity in the dynamo region of extrasolar planets remains to be evaluated. According to Nellis (2000), the electrical conductivity remains constant throughout the metallic region. For this reason, it is not necessary to average over the volume of the conducting region.

Because the magnetic moment scaling is applied relative to Jupiter, only the relative value of the conductivity, i.e. $\sigma / \sigma_{\mathrm{J}}$ is required. In this work, the conductivity is assumed to be the same for extrasolar gas giants as for Jupiter, i.e. $\sigma / \sigma_{\mathrm{J}}=1$.

\subsubsection{Known planetary parameters}

For the estimation of the planetary magnetic moment with the scaling laws (4.36) to (4.39), the size of the dynamo region $r_{\mathrm{c}}$, the density within the dynamo region $\rho_{\mathrm{c}}$ and the planetary rotation rate $\omega$ are required. In Sections 4.3.2 to 4.3.4 it was shown that these values can be constructed from the planetary mass $M_{\mathrm{p}}$, the planetary radius $R_{\mathrm{p}}$ and the orbital frequency $\omega_{\text {orbit }}$ of the planet. These values were already presented and discussed in Section 2.6.2.

Table 4.3 lists the values of the observed and deduced planetary parameters. Most of the quantities in Table 4.3 are given in units normalised to Jupiter (denoted by subscript J), with $R_{\mathrm{J}}=71492 \mathrm{~km}$ (Cain et al. 1995), $M_{\mathrm{J}}=1.9 \cdot 10^{27} \mathrm{~kg}\left(\right.$ Raith 1997), and $\omega_{\mathrm{J}}=$ $1.77 \cdot 10^{-4} \mathrm{~s}^{-1}$ (Raith 1997). The planetary radius $R_{\mathrm{p}}$ and mass $M_{\mathrm{p}}$ are directly taken from measurements (see Section 2.6.2). Assuming tidal locking, the rotation rate $\omega$ is set equal to the orbital period $\omega_{\text {orbit }}$, which is also known from observations (see Section 
2.6.2). For Jupiter and Saturn, the measured planetary rotation rates are used instead. The fractional size $r_{\mathrm{c}} / R_{\mathrm{p}}$ of the dynamo region is obtained using eq. (4.56) and (4.62) from Section 4.3.2. This corresponds to Model 4 of Table 4.1. This value is then used together with eq. (4.68) to determine the core density $\rho_{\mathrm{c}}$. Again, in Table 4.2 of Section 4.3.3, this corresponds to Model 4.

For each of the planets in Table 4.3 , the upper and lower limit for the magnetic moment $\mathcal{M}$ will be calculated in Section 4.4.

\begin{tabular}{|l||c|c||c|c|c|}
\hline Planet & $R_{\mathrm{p}}\left[R_{\mathrm{J}}\right]$ & $M_{\mathrm{p}}\left[M_{\mathrm{J}}\right]$ & $\omega\left[\omega_{\mathrm{J}}\right]$ & $r_{\mathrm{c}} / R_{\mathrm{p}}$ & $\rho_{\mathrm{c}}\left[\rho_{\mathrm{c}, \mathrm{J}}\right]$ \\
\hline \hline Jupiter & 1.0 & 1.0 & 1.0 & 0.85 & 1.0 \\
Saturn & 0.84 & 0.3 & 0.93 & 0.72 & 0.65 \\
HD 209458b & $1.42^{a}$ & $0.69^{a}$ & $0.12^{a}$ & 0.44 & 0.44 \\
OGLE-TR-10b & $1.24^{b}$ & $0.57^{b}$ & $0.13^{b}$ & 0.55 & 0.49 \\
OGLE-TR-56b & $1.25^{c}$ & $1.18^{c}$ & $0.34^{c}$ & 0.76 & 0.72 \\
OGLE-TR-111b & $1.0^{d}$ & $0.53^{d}$ & $0.10^{d}$ & 0.73 & 0.66 \\
OGLE-TR-113b & $1.09^{b}$ & $1.29^{b}$ & $0.29^{e}$ & 0.85 & 1.0 \\
OGLE-TR-132b & $1.13^{f}$ & $1.19^{f}$ & $0.24^{f}$ & 0.82 & 0.88 \\
TrES-1b & $1.04^{g}$ & $0.76^{g}$ & $0.14^{h}$ & 0.78 & 0.77 \\
$\tau$ Bootes b (light) & $1.2^{i}$ & $4.4^{i}$ & $0.12^{j}$ & 0.94 & 2.07 \\
$\tau$ Bootes b (medium) & $1.58^{i}$ & $7.0^{i}$ & $0.12^{j}$ & 0.91 & 1.54 \\
$\tau$ Bootes b (heavy) & $1.48^{i}$ & $10.0^{i}$ & $0.12^{j}$ & 0.95 & 2.43 \\
\hline
\end{tabular}

Table 4.3: Parameters for different "Hot Jupiters" (Jupiter and Saturn are shown for comparison). The values used for the calculation of the magnetic moments are given in units normalised to Jupiter, except for the radius of the dynamo region, which is given in terms of the respective planetary radius. Sources of the exoplanetary parameters: ${ }^{(a)}$ Cody and Sasselov (2002), ${ }^{(b)}$ Konacki et al. (2005), ${ }^{(c)}$ Bouchy et al. (2005), ${ }^{(d)}$ Pont et al. (2004), ${ }^{(e)}$ Konacki et al. (2004), ${ }^{(f)}$ Moutou et al. (2004), ${ }^{(g)}$ Sozzetti et al. (2004), ${ }^{(h)}$ Alonso et al. (2004), ${ }^{(i)}$ see text, ${ }^{(j)}$ Leigh et al. (2003).

\subsection{Scaling results for gas giants}

Combining the magnetic moment scaling relations (4.36), (4.37), (4.38) and (4.39) with the estimates for the planetary parameters given in Table 4.3 , the magnetic moments of different Hot Jupiters are evaluated and given in units of Jupiter's current magnetic moment, $\mathcal{M}_{\mathrm{J}}=1.56 \cdot 10^{27} \mathrm{Am}^{2}$ (Cain et al. 1995). A similar approach was already taken by Farrell et al. (1999) and Grießmeier et al. (2004). In the present work, however, the planetary parameters are evaluated more precisely.

For each set of parameters in Table 4.3, the four scaling relations (4.36), 4.37, , 4.38) and (4.39) are evaluated. Of the four results, the smallest and the largest magnetic moments are given in the left column of Table 4.4. Obviously, the results of the different 
scaling laws are not identical, but they are approximately of the same order of magnitude. In no case, the lower and the upper limit differ by more than a factor of 4 . The middle and right column of Table 4.4 give the magnetic moment of a Hot Jupiter with doubled rotation rate $\omega$ (i.e. $\mathcal{M}_{\mathrm{dr}}$ ) and for the hypothetical case where the planet is not tidally locked, but rapidly rotating (i.e. $\mathcal{M}_{\text {rr }}$ ), respectively. See the discussion below for these cases.

\begin{tabular}{|l||c|c|c|}
\hline Planet & $\mathcal{M}\left[\mathcal{M}_{\mathrm{J}}\right]$ & $\begin{array}{c}\mathcal{M}_{\mathrm{dr}}\left[\mathcal{M}_{\mathrm{J}}\right] \\
\text { (doubled rotation) }\end{array}$ & $\begin{array}{c}\mathcal{M}_{\text {rr }}\left[\mathcal{M}_{\mathrm{J}}\right] \\
\text { (rapidly rotating) }\end{array}$ \\
\hline \hline Jupiter & 1.0 & - & 1.0 \\
Saturn & $0.19 \ldots 0.28$ & - & $0.21 \ldots 0.29$ \\
HD 209458b & $0.024 \ldots 0.092$ & $0.047 \ldots 0.13$ & $0.19 \ldots 0.26$ \\
OGLE-TR-10b & $0.038 \ldots 0.13$ & $0.077 \ldots 0.19$ & $0.29 \ldots 0.36$ \\
OGLE-TR-56b & $0.45 \ldots 0.71$ & $0.90 \ldots 1.0$ & $1.2 \ldots 1.3$ \\
OGLE-TR-111b & $0.045 \ldots 0.17$ & $0.090 \ldots 0.23$ & $0.45 \ldots 0.52$ \\
OGLE-TR-113b & $0.39 \ldots 0.69$ & $0.77 \ldots 0.98$ & $1.3 \ldots 1.4$ \\
OGLE-TR-132b & $0.31 \ldots 0.60$ & $0.62 \ldots 0.85$ & $1.2 \ldots 1.3$ \\
TrES-1b & $0.10 \ldots 0.29$ & $0.20 \ldots 0.41$ & $0.75 \ldots 0.78$ \\
$\tau$ Bootes b (light) & $0.48 \ldots 1.2$ & $0.95 \ldots 1.7$ & $3.3 \ldots 4.4$ \\
$\tau$ Bootes b (medium) & $0.98 \ldots 2.1$ & $2.0 \ldots 3.0$ & $6.0 \ldots 10$ \\
$\tau$ Bootes b (heavy) & $1.1 \ldots 2.5$ & $2.2 \ldots 3.5$ & $7.0 \ldots 12$ \\
\hline
\end{tabular}

Table 4.4: Results for different "Hot Jupiters" (Jupiter and Saturn are shown for comparison). Left column: Expected planetary magnetic moment $\mathcal{M}$. Middle column: magnetic moment $\mathcal{M}_{\mathrm{dr}}$ for planets with twice the rotation rate of Table 4.3 ("doubled rotation", imperfect tidal locking case). Right column: magnetic moment $\mathcal{M}_{\mathrm{rr}}$ for a hypothetical planet not subject to tidal locking ("rapidly rotating", i.e. with $\omega=\omega_{\mathrm{J}}$ ).

In Table 4.4(left column), different "classes" of planets can be distinguished:

- Jupiter: The result for Jupiter is correct by construction. Nothing can be inferred from this result.

- Saturn: For Saturn, the scaling laws yield a magnetic moment in the range of $0.19 \mathcal{M}_{\mathrm{J}} \leq \mathcal{M}_{\mathrm{S}} \leq 0.28 \mathcal{M}_{\mathrm{J}}$. This has to be compared to the observed value of $\mathcal{M}_{\mathrm{S}}=0.03 \mathcal{M}_{\mathrm{J}}$ (calculated from Cain et al. 1995 ${ }^{12}$. This large discrepancy should not, however, be attributed to the scaling laws. In fact, for Saturn, the assumption of hydrostatic equilibrium is justified, but the approximation of a polytropic index of $\kappa=1$ is not valid. This is explained by the existence of a large rocky core, see Section 4.3.1. For this reason, the parameters $r_{\mathrm{c}}$ and $\rho_{\mathrm{c}}$ are overestimated in this simple calculation, and the scaling relations result in a magnetic moment larger

\footnotetext{
${ }^{12}$ Recent measurements by the Cassini spacecraft are consistent with this value (Dougherty et al. 2005).
} 
than the real value. Note that even in this case, where one of the approximations is not fulfilled, the error in $\mathcal{M}$ does not exceed one order of magnitude.

- "Hot Jupiters": The planets HD 209458b, OGLE-TR-10b, OGLE-TR-111b and TrES-1b, with orbital distances of about $0.04 \mathrm{AU}$, have low rotation rates, resulting in very weak magnetic moments.

- "Very Hot Jupiters": The planets OGLE-TR-56b, OGLE-TR-113b and OGLE-TR$132 \mathrm{~b}$ orbit their host stars at even closer distances ( $\sim 0.02 \mathrm{AU})$. According to Kepler's law, eq. (3.1), this results in an increased orbital revolution rate. Because the planets are synchronously rotating (see Section 3.1), this implies a higher planetary rotation rate, which leads to a higher planetary magnetic dipole moment.

- "Heavy Hot Jupiters": The planet $\tau$ Bootes b, although it is a Hot Jupiter, differs from the other planets considered here insofar as its higher mass leads to an increased magnetic moment. Only a lower limit is known for the planetary mass, leading to a lower limit for the magnetic moment. In any case, the magnetic moment is expected to be larger than that of Jupiter. Some publications give larger numbers for the magnetic moment of $\tau$ Bootes (e.g. up to $3.8 \mathcal{M}_{\mathrm{J}}$, Farrell et al. 1999). For heavier planets, the difference to the numbers given here is even more pronounced, with magnetic moments of up to $316 \mathcal{M}_{\mathrm{J}}$ for the $10 M_{\mathrm{J}}$ planet HD 114762b (Farrell et al. 1999) or, for example, of $69 \mathcal{M}_{\mathrm{J}}$ for the $12.7 M_{\mathrm{J}}$ planet HD 38529c (Lazio et al. 2004). These large values, however, are reached only because Blackett's scaling law (see Section 4.1.1) is used, and because condition (4.60) is violated. Using other scaling laws and a realistic value for the size of the dynamo region $r_{\mathrm{c}}$, the magnetic moment of $\tau$ Bootes $\mathrm{b}$ is found to be not more than a few times the value of Jupiter, even for the case where the planetary mass is equal to 10 $M_{\mathrm{J}}$.

It is important to note that "Hot Jupiters" (like, for example, HD 209458b) have very low planetary magnetic dipole moments. The smallness of these values is caused by the slow rotation of the planet. It is instructive to compare the planetary magnetic moment $\mathcal{M}$ to the magnetic moment of a totally identical, but rapidly rotating planet (i.e. not subject to tidal locking, with $\omega=\omega_{\mathrm{J}}$ ). These values (denoted by $\mathcal{M}_{\mathrm{rr}}$ ) are given in the right column of Table 4.4. Note that these numbers do not necessarily represent realistic situations. They are given to illustrate the effect of tidal locking on the magnetic moment. It can be seen that for Hot Jupiters, tidal locking reduces the upper limit of the magnetic moment by a factor of about 3 , while the lower limit is reduced by a factor of about 7 . The fact that the reduced rotation of tidally locked planets leads to smaller magnetic moments can also be seen from the scaling relations 4.36 to 4.39). It is clear that the magnetic moment reduction by tidal locking should be less than or equal to the ratio $\omega / \omega_{\mathrm{J}}$ from Table 4.3 .

The difference in the magnetic moments of "Very Hot Jupiters" and "Hot Jupiters" is a result of the different distances to the star. The dependency of minimum and maximum magnetic moment on the orbital distance is shown in Figure 4.5 for a Jupiter-like planet orbiting a Sun-like star. The area between minimum and maximum moment is shaded in grey. For large orbital distances, where the tidal locking timescale is very long (i.e. $\tau_{\text {sync }}>10 \mathrm{Gyr}$ ), the planetary rotation rate is taken to be constant and identical to Jupiter's 


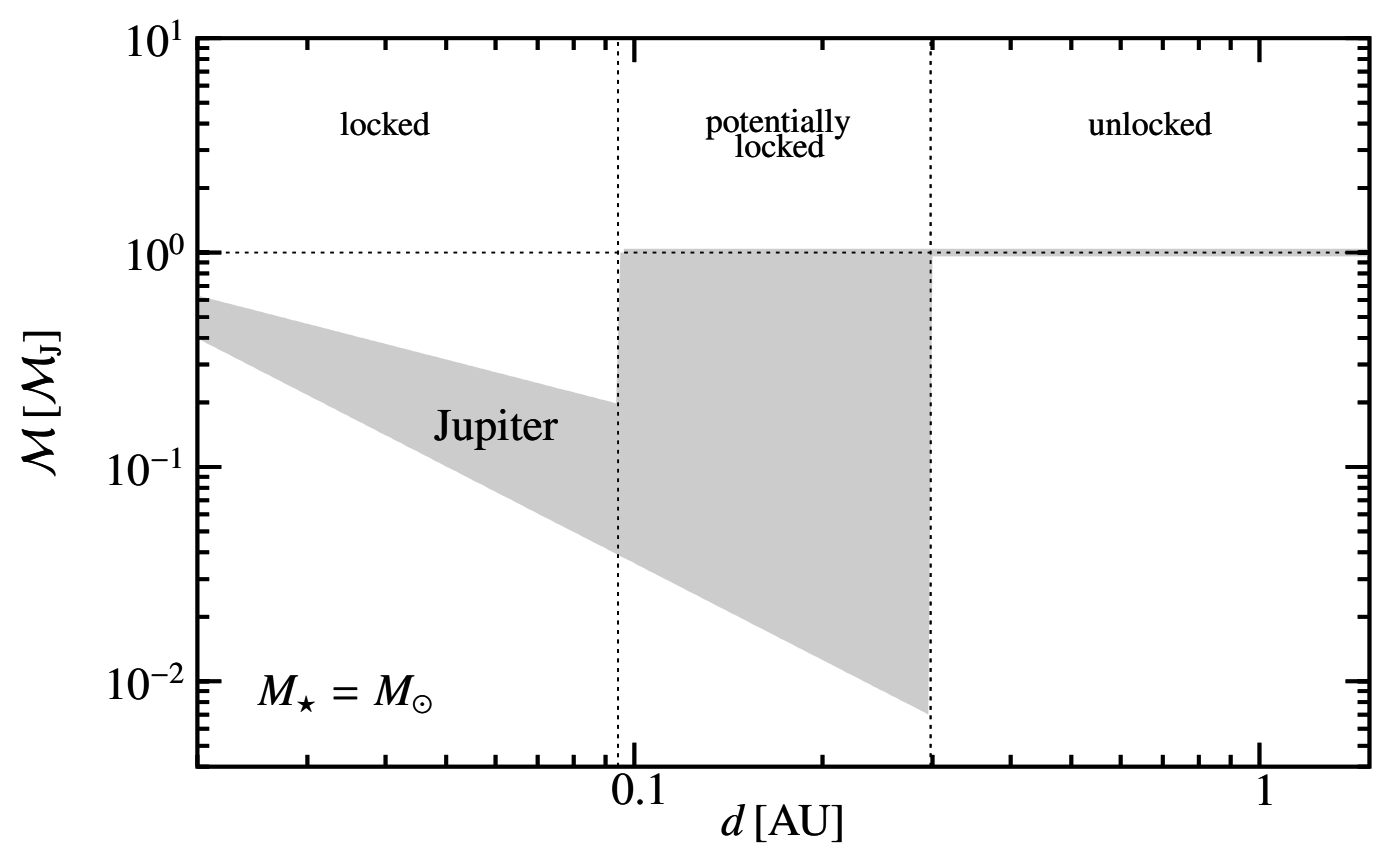

Figure 4.5: Estimated magnetic moment for a planet identical to Jupiter in an orbit around a Sunlike star as a function of orbital distance. The magnetic moment is given in units of Jupiter's current magnetic moment.

rotation rate (i.e. $\omega=\omega_{\mathrm{J}}$ ). Because all parameters are equal to those of Jupiter in this case, all magnetic moment scaling laws yield the same result $\left(\mathcal{M}=\mathcal{M}_{\mathrm{J}}\right)$ in this case. For small orbital distances (corresponding to $\tau_{\text {sync }}<0.1 \mathrm{Gyr}$ ) tidal locking sets in and $\omega$ is given by equation (3.1), leading to a dependency on the semi-major axis of the orbit. Also, eq. (3.1) shows that the mass of the star is an important parameter in the tidally locked regime. For intermediate distances, it is not certain whether an observed planet will be subject to tidal locking or not $\left(0.1 \mathrm{Gyr}<\tau_{\text {sync }}<10 \mathrm{Gyr}\right)$, and both cases have to be considered. For this reason, in the "potentially locked" regime, the lower limit for $\mathcal{M}$ is calculated with the rotation rate of a tidally locked planet, and the upper limit for $\mathcal{M}$ with the rotation rate of Jupiter. It can be seen that a tidally locked planet has a strongly reduced magnetic moment when compared to a freely rotating planet (i.e. a planet with a large orbital distance). One can also note the influence of the orbital distance on the magnetic moment of tidally locked planets.

Similarly to Figure 4.5, Figures 4.6 and 4.7 show the dependence of the magnetic moment $\mathcal{M}$ on the orbital distance $d$ for planets identical to HD 209458b and $\tau$ Bootes b, respectively. For Figure 4.6, a stellar mass of $1.06 M_{\odot}$ is assumed (Cody and Sasselov 2002), and for Figure 4.7 a stellar mass of $1.42 M_{\odot}$ (Fuhrmann et al. 1998) is taken. In the unlocked regime (i.e. for large orbital distances), the magnetic moment corresponds to $\mathcal{M}_{\text {rr }}$ of Table 4.4. The comparison of the results for the different planets clearly shows that for exoplanets with a large mass and radius, a larger magnetic moment can be expected. The stellar mass influences the planetary magnetic moment via the rotation rate of a tidally locked planet, see eq. (3.1).

As discussed in Section 3.1.2, "Hot Jupiters" should not be expected to be exactly 


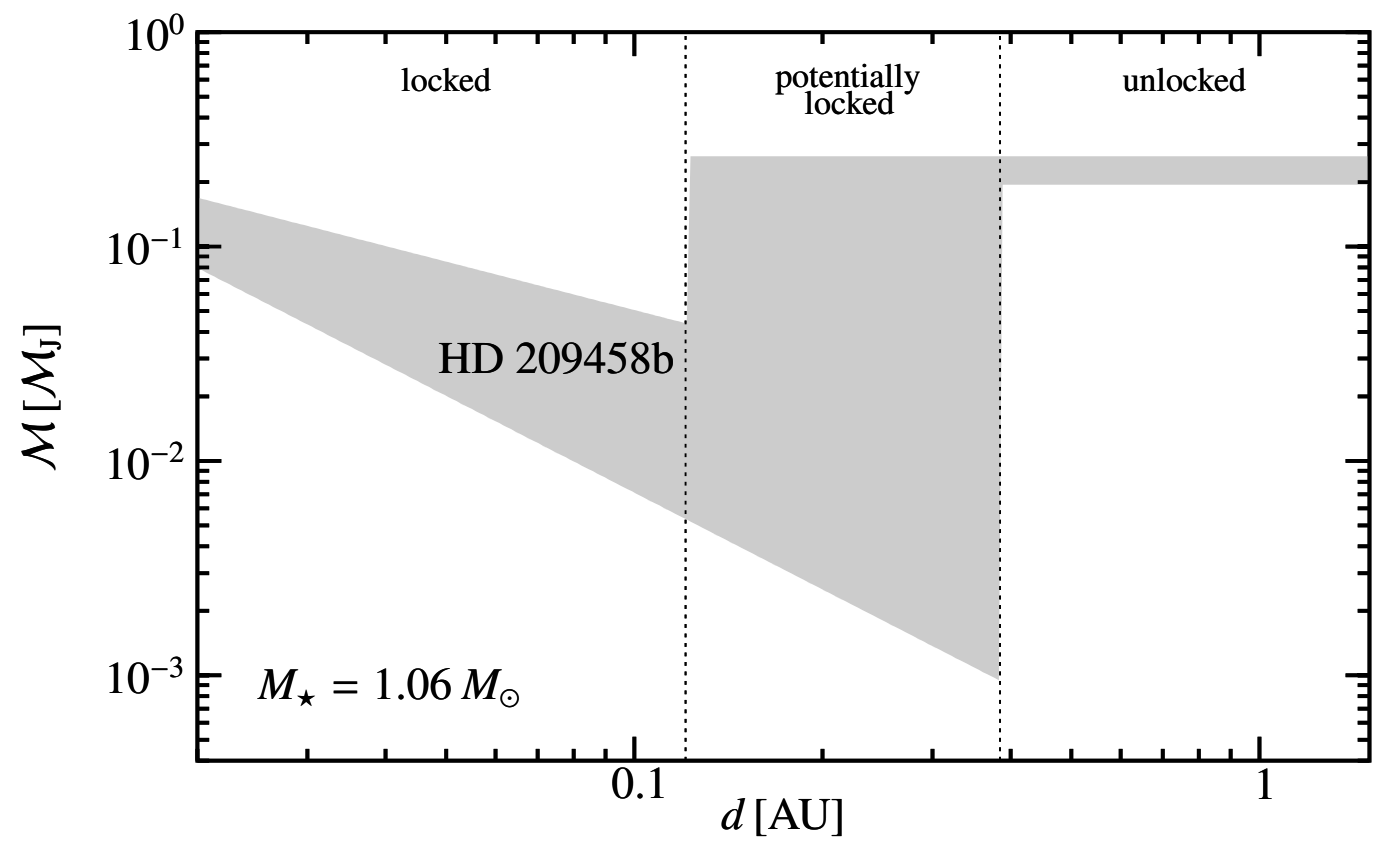

Figure 4.6: Estimated magnetic moment for a typical "Hot Jupiter" (HD 209458b) orbiting a star with mass $M_{\star}=1.06 M_{\odot}$ as a function of orbital distance. The magnetic moment is given in units of Jupiter's current magnetic moment.

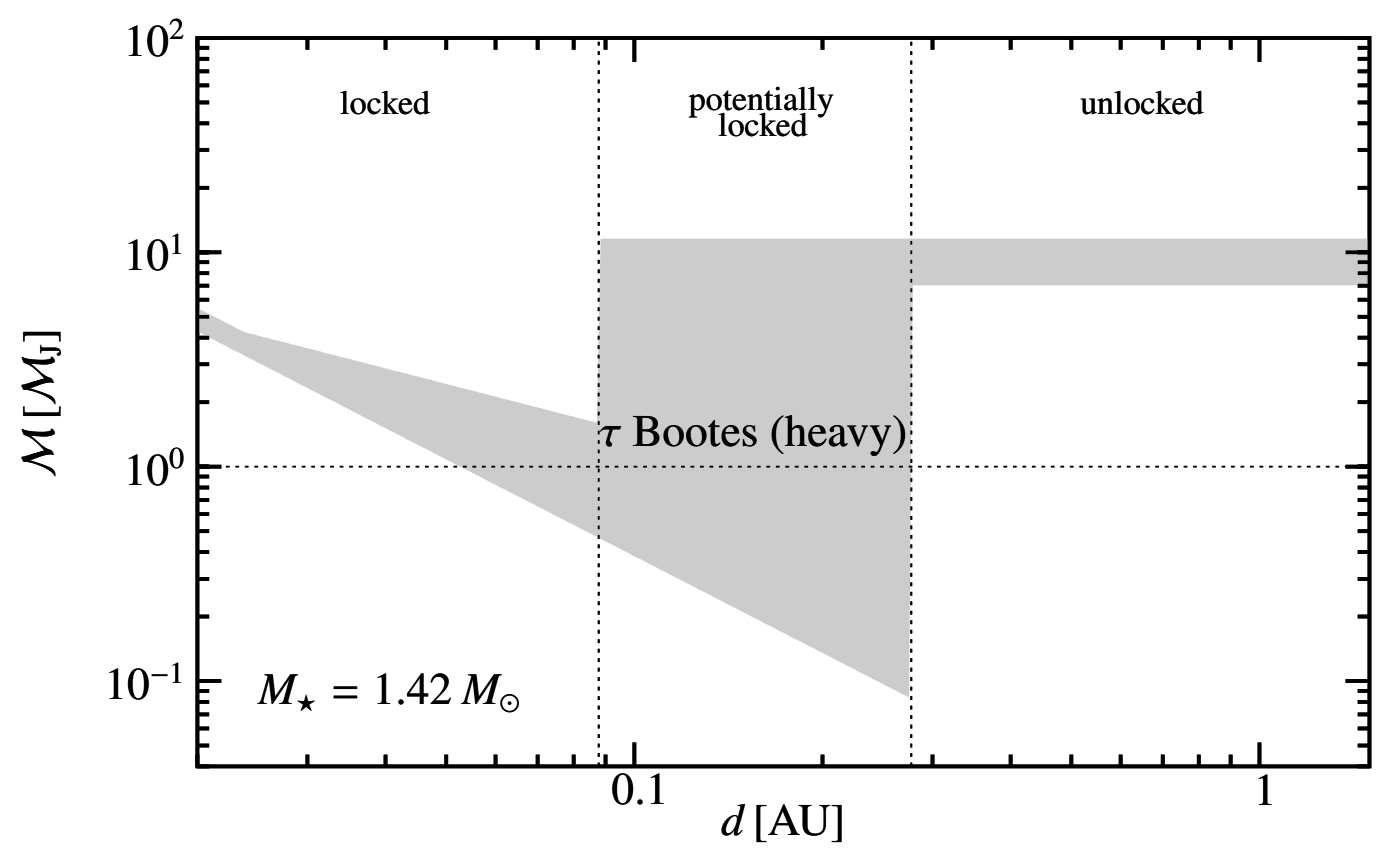

Figure 4.7: Estimated magnetic moment for a "Heavy Hot Jupiter" ( $\tau$ Bootes, heavy model) orbiting a star with mass $M_{\star}=1.42 M_{\odot}$ as a function of orbital distance. The magnetic moment is given in units of Jupiter's current magnetic moment. 
tidally locked. The reason is that thermal atmospheric tides caused by the strong stellar irradiation can drive planets away from synchronous rotation (Showman and Guillot 2002, Laskar and Correia 2004). Showman and Guillot (2002) estimate that this effect introduces an additional uncertainty of a factor of 2 for the planetary rotation rate $\omega$. As shown in the middle column of Table 4.4, this doubles the lower limit for the magnetic moment, but the influence on the upper limit of $\mathcal{M}$ is weak.

The smallness of the planetary magnetic field due to tidal locking has important consequences for the planetary magnetosphere. This will be discussed in Section 5.3.3. Before turning to other subjects, however, the magnetic moment analysis is repeated for terrestrial planets.

\subsection{Input parameters for terrestrial planets}

While currently over 150 giant exoplanets are known, Earth-like planets outside the solar system are not yet accessible to current detection techniques (see Section 2.6.3). With no detected Earth-sized rocky ("terrestrial") planets, planetary models have to be used, and the size of the corresponding magnetic moment has to be estimated from the scaling laws (4.27), (4.32), (4.33) and (4.34) given in Section 4.1.9. This section presents the input parameters required for this estimation.

\subsubsection{Planetary models}

For terrestrial planets, structural models are more complicated than for gaseous giants. Usually, three-layered models are used. Within this work, only terrestrial planets for which structure models exist will be studied. However, the orbital distance of the planet and the mass of its host star will be treated as a free parameter. As described in Section 2.6.4, the following model planets will be studied:

- exact analogues to the Earth,

- Mercury-like planets,

- large terrestrial planets,

- large ocean planets.

The radii and masses of these model planets are given in Table 4.6. For these four cases, the planetary magnetic moment will be estimated using the parameters collected in the following sections.

\subsubsection{Size of the dynamo region $r_{\mathrm{C}}$}

In contrast to Curtis and Ness (1986) or Grießmeier et al. (2004), where the size of the dynamo region $r_{\mathrm{c}}$ is estimated from the planetary mass (and radius), or the discussion of Section 4.3, where it is deduced from a given model for the planetary structure, the values for $r_{\mathrm{c}}$ of terrestrial planets are taken from existing planetary models (Cain et al. 1995, Léger et al. 2004). The values used for the different cases are given in Table 4.6. 
For Earth and Mercury, the size of the dynamo region is taken to be the outer limit of the metallic core as given by Cain et al. (1995). For the case of the "Large Earth" and for the "Ocean Planet", the size of the core is taken from Léger et al. (2004).

\subsubsection{Density of the dynamo region $\rho_{\mathrm{c}}$}

Similarly, the density within the dynamo region, $\rho_{\mathrm{c}}$, is taken from published models. For the cases treated in the following, the values of $\rho_{\mathrm{c}}$ are given in Table 4.6. For Earth and Mercury, the average core density is taken from Cain et al. (1995). For the "Large Earth" and the "Ocean Planet" case, the density at the outer boundary of the metallic core is taken from Léger et al. (2004).

\subsubsection{Planetary rotation rate $\omega$}

As currently no Earth-like terrestial planets are known, different hypothetical planets will be examined. Both the orbital distance of the planet as well as the mass of the host star will be treated as free parameters. The timescale for tidal locking (as discussed in Section 3.1.1 and thus the planetary rotation rate $\omega$ depend on these parameters. The same three cases as in Section 4.3.4 have to be considered. The approach for the cases, however, differs slightly:

1. For planets at small enough distances such that the timescale for tidal locking is small (i.e. $\tau_{\text {sync }} \leq 100 \mathrm{Myr}$ ), the rotation period is taken to be synchronised with the orbital period $\left(\omega=\omega_{\mathrm{f}} \approx \omega_{\text {orbit }}\right.$ ). For terrestrial planets, the orbital frequency $\omega_{\text {orbit }}$ is calculated from the Keplerian orbit, see eq. (3.1). For this reason, the rotation rate depends on the orbital distance $d$ as well as on the stellar mass $M_{\star}$. One has to keep in mind that, because of thermal atmospheric tides, tidal interaction will not lead to perfectly synchronous rotation (see Section 3.1.2). For this reason, both the cases $\omega_{\mathrm{f}}=\omega_{\text {orbit }}$ and $\omega_{\mathrm{f}}=2 \omega_{\text {orbit }}$ will be studied.

2. Planets with distances resulting in $100 \mathrm{Myr} \leq \tau_{\text {sync }} \leq 10$ Gyr may or may not be tidally locked. This will, for example, depend on the exact age of the planetary system, which is typically in the order of a few Gyr. For this reason, the upper limit for the magnetic moment of such a planet is calculated without tidal locking (see below), and the lower limit for the magnetic moment is calculated with tidal locking (i.e. $\omega=\omega_{\mathrm{f}}$ ). Note that this area is maximised by taking the upper limit for the initial rotation rate $\omega_{\mathrm{i}}$ to calculate the border to the tidally locked regime, while the lower limit for $\omega_{\mathrm{i}}$ is assumed to determine the border to the freely rotating regime (see below).

3. For planets far away from the central star, the timescale given by eq. (3.7) is very large. For planets with $\tau_{\text {sync }} \geq 10 \mathrm{Gyr}$, the effect of tidal interaction can be neglected, and the planetary rotation rate can be assumed to be equal to the initial rotation rate $\omega_{\mathrm{i}}$. However, The initial rotation rate $\omega_{\mathrm{i}}$ of a terrestrial planet is a poorly known quantity (see, e.g. Correia and Laskar 2003a). It will certainly depend on the details of planetary formation and can be strongly influenced by processes like migration or impacts. Therefore, two limits are considered for $\omega_{\mathrm{i}}$ : 
- a relatively high initial rotation rate as suggested for the early Earth-Moon system, corresponding to a length of day of $13.1 \mathrm{~h}$ (MacDonald 1964) and

- a lower rotation rate corresponding to a day of $30 \mathrm{~h}$.

Note that a primordial rotation period of the order of 10 hours is consistent with the relation between the planetary angular momentum density and planetary mass observed in the solar system (Hubbard 1984, Chapter 4).

Table 4.5 gives the rotation rate $\omega$ for tidally locked extrasolar planets in different orbits around stars of different masses relative to the rotation rate of the Earth, $\omega_{\mathrm{E}}$ (with $\omega_{\mathrm{E}}=7.27 \cdot 10^{-5} \mathrm{~s}^{-1}$ ). The values were calculated according to Kepler's law, eq. 3.1. The effect of (imperfect) tidal locking on the expected magnetic moment of terrestrial extrasolar planets will be studied in Section 4.6 .

\begin{tabular}{|l||c|c|c|c|}
\hline & $M_{\star}=0.1 M_{\odot}$ & $M_{\star}=0.2 M_{\odot}$ & $M_{\star}=0.5 M_{\odot}$ & $M_{\star}=1.0 M_{\odot}$ \\
\hline \hline $0.05 \mathrm{AU}$ & $0.08^{a}$ & 0.11 & 0.17 & 0.24 \\
$0.1 \mathrm{AU}$ & 0.03 & $0.04^{a}$ & 0.06 & 0.09 \\
$0.2 \mathrm{AU}$ & $0.010^{b}$ & 0.014 & $0.02^{a}$ & 0.03 \\
$0.5 \mathrm{AU}$ & $0.002^{c}$ & $0.003^{b}$ & $0.005^{b}$ & $0.008^{b}$ \\
\hline
\end{tabular}

Table 4.5: Planetary rotation rates of tidally locked planets relative to the Earth's current rotation rate $\left(\omega / \omega_{\mathrm{E}}\right)$. Notes: ${ }^{(a)}$ located within the habitable zone (see Section 2.3, , ${ }^{(b)}$ tidal locking possible, but not certain for an Earth-like planet, ${ }^{(c)}$ tidal locking unlikely for an Earth-like planet.

\subsubsection{Conductivity within the dynamo region $\sigma$}

Because the magnetic moment scaling is applied relative to Earth, only the relative value of the conductivity, i.e. $\sigma / \sigma_{\mathrm{E}}$ is required. In the following, the conductivity is assumed to be similar for all terrestrial planets, i.e. $\sigma / \sigma_{\mathrm{E}}=1$.

\subsubsection{Planetary structure}

Table 4.6 lists the planetary parameters required for the estimation of the planetary magnetic dipole moment. The planetary mass $M_{\mathrm{p}}$ and radius $R_{\mathrm{p}}$ (which were already presented in Section 2.6.4) as well as the core density $\rho_{\mathrm{c}}$ are given in units normalised to Earth (denoted by subscript E), with $R_{\mathrm{E}}=6371 \mathrm{~km}$ (Cain et al. 1995$), M_{\mathrm{E}}=6.0 \cdot 10^{24}$ $\mathrm{kg}\left(\right.$ Raith 1997), and $\rho_{\mathrm{c}, \mathrm{E}}=10.615 \cdot 10^{3} \mathrm{~kg} / \mathrm{m}^{-3}$ (calculated from Cain et al. 1995). The size of the core, $r_{\mathrm{c}}$, is given in fractions of the planetary radius $R_{\mathrm{p}}$.

\subsection{Scaling results for terrestrial planets}

Similarly to Section 4.4, the different magnetic moment scaling relations 4.36, (4.37), (4.38) and (4.39) are used together with the planetary parameters from Table 4.6 to obtain 


\begin{tabular}{|l||c|c||c|c|}
\hline Planet & $R_{\mathrm{p}}\left[R_{\mathrm{E}}\right]$ & $M_{\mathrm{p}}\left[M_{\mathrm{E}}\right]$ & $r_{\mathrm{c}} / R_{\mathrm{p}}$ & $\rho_{\mathrm{c}}\left[\rho_{\mathrm{c}, \mathrm{E}}\right]$ \\
\hline \hline Earth & 1.0 & 1.0 & $0.55^{a}$ & 1.0 \\
Mercury & $0.38^{a}$ & $0.055^{b}$ & $0.72^{a}$ & $0.72^{a}$ \\
Large Earth & $1.63^{c}$ & $6.0^{c}$ & $0.52^{c}$ & $1.46^{c}$ \\
Ocean Planet & $2.0^{c}$ & $6.0^{c}$ & $0.35^{c}$ & $1.47^{c}$ \\
\hline
\end{tabular}

Table 4.6: Parameters for different terrestrial planets. The values used for the calculation of the magnetic moments are given in units normalised to Earth, except for the radius of the dynamo region, which is represented in terms of the respective planetary radius. Sources of the parameters: (a) Cain et al. (1995), ${ }^{(b)}$ Raith (1997), ${ }^{(c)}$ Léger et al. (2004).

an estimation for the planetary magnetic dipole moment. For terrestrial planets, however, all values in the scaling relations are normalised to Earth. Accordingly, the resulting magnetic moments are given in units of Earth's current magnetic moment, $\mathcal{M}_{\mathrm{E}}=7.91$. $10^{22} \mathrm{Am}^{2}$ (Cain et al. 1995).

The minimum and maximum value obtained from the four scaling laws are shown in the left column of Table 4.7. Similarly to Section 4.4, the results obtained from the different scaling laws are not identical. Because some of the parameters (mainly $\omega$ ) deviate considerably from the value of the current Earth (see, e.g. Table 4.5), the resulting magnetic moments are more dissimilar than the values found for gas giants in Section 4.4. With the largest differences reaching a factor of 7 , it is still possible to give the order of magnitude of the resulting magnetic moments. The middle and right column of Table 4.7 give the magnetic moments of planets with doubled rotation rate $\omega$ (i.e. $\mathcal{M}_{\mathrm{dr}}$ ) and for the hypothetical case where the planet is not tidally locked, but rapidly rotating (i.e. $\mathcal{M}_{\text {rr }}$ ), respectively. See the discussion below for these cases.

The left column of Table 4.7 shows the magnetic moments one can expect for terrestrial planets which are tidally locked at $0.2 \mathrm{AU}$ around an $\mathrm{M}$ type star of 0.5 solar masses. The results for the different cases are as follows:

- Earth-like: The magnetic moment of a tidally locked twin of the planet Earth is strongly reduced. Besides the rotation rate, all parameters were kept constant, thus demonstrating the influence of tidal locking on the planetary magnetic dipole moment.

- Mercury-like: The calculated value for the magnetic moment should not be compared to the value observed for Mercury. The reason is that in Table 4.7, the planet is assumed to orbit a star with 0.5 solar masses at a distance of $0.2 \mathrm{AU}$. If the calculation is repeated for a star with solar mass and setting the orbital distance to 0.4 $\mathrm{AU}$, the magnetic moment is found to be in the range $5.6 \cdot 10^{-4} \ldots 1.1 \cdot 10^{-2}$. This is in agreement with the observed value of $6.3 \cdot 10^{-4} \mathcal{M}_{\mathrm{E}}$ (calculated from Cain et al. 1995).

If one compares the result for Earth-like and Mercury-like tidally locked planets, Table 4.7 demonstrates the importance of a large planetary size for the generation of a substantial magnetic moment. 


\begin{tabular}{|l||c|c|c|}
\hline Planet & $\mathcal{M}\left[\mathcal{M}_{\mathrm{E}}\right]$ & $\begin{array}{c}\mathcal{M}_{\mathrm{dr}}\left[\mathcal{M}_{\mathrm{E}}\right] \\
\text { (doubled rotation) }\end{array}$ & $\begin{array}{c}\mathcal{M}_{\mathrm{rr}}\left[\mathcal{M}_{\mathrm{E}}\right] \\
\text { (rapidly rotating) }\end{array}$ \\
\hline \hline Earth-like & $0.022 \ldots 0.15$ & $0.043 \ldots 0.21$ & $0.8 \ldots 1.8$ \\
\hline $\begin{array}{r}\text { Mercury-like } \\
\text { (locked) }\end{array}$ & $0.0011 \ldots 0.14$ & $0.0022 \ldots 0.14$ & $0.041 \ldots 0.14$ \\
\hline $\begin{array}{r}\text { Large Earth }^{b} \\
\text { (locked) }^{b}\end{array}$ & $0.0011 \ldots 0.015$ & $0.0022 \ldots 0.022$ & $0.041 \ldots 0.14$ \\
\hline Ocean Planet & $0.12 \ldots 12.5$ & $0.24 \ldots 12.5$ & $4.0 \ldots 12.5$ \\
& $0.061 \ldots 0.37$ & $0.12 \ldots 0.52$ & $2.2 \ldots 5.8$ \\
\hline
\end{tabular}

Table 4.7: Results for different terrestrial planets at $0.2 \mathrm{AU}$ around a star with $0.5 M_{\odot}$. Left column: Expected planetary magnetic moment $\mathcal{M}$. Middle column: Magnetic moment $\mathcal{M}_{\mathrm{dr}}$ for planets with twice the rotation rate of Table 4.5 ("doubled rotation", imperfect tidal locking case). Right column: Magnetic moment $\mathcal{M}_{\text {rr }}$ for a hypothetical planet not subject to tidal locking ("rapidly rotating", i.e. with $\omega=\omega_{\mathrm{E}}$ ). Notes: ${ }^{(a)}$ For distances $d \geq 0.17$ AU, Mercury falls into the "potentially locked" regime. Thus, the upper limit for $\mathcal{M}$ is identical to that of $\mathcal{M}_{\mathrm{rr}}$. Values for a Mercury-like planet still tidally locked at $0.2 \mathrm{AU}$ are given for comparison. ${ }^{(b)}$ For distances $d \geq 0.197 \mathrm{AU}$, a Large Earth falls into the "potentially locked" regime. Thus, the upper limit for $\mathcal{M}$ is identical to that of $\mathcal{M}_{\text {rr }}$. Values for a Large Earth still tidally locked at $0.2 \mathrm{AU}$ are given for comparison.

- "Large Earth": Because of its relatively large metallic core, a larger planet is still able to sustain a considerable magnetic moment, even when its rotation rate is strongly reduced. Because of the larger planetary radius, however, this implies a surface magnetic field very similar in strength to that of the "Earth" case.

- "Ocean Planet": Although being identical in mass to the "Large Earth" case, the "Ocean Planet" has a slightly smaller core (see Table 4.6), leading to a smaller magnetic moment. Also, the average density is lower, and the planetary radius is larger, such that the surface magnetic field is considerably lower than in the "Earth" and "Large Earth" case.

As in Section 4.4, the influence of tidal locking on the planetary magnetic moment is examined. In Table 4.7, the right column contains the magnetic moment of a (hypothetical) rapidly rotating planet, $\mathcal{M}_{\mathrm{rr}}$. Note that this case is not realistic (unless the planet was brought to this position only recently and the time it has spent at this position is short compared to the tidal locking timescale of Section 3.1.1, but it is instructive to compare the influence of tidal locking on the planetary magnetic moment. Similarly to Section 4.4, the lower limit for the magnetic moment is reduced by approximately the same factor as the rotation rate (see Table 4.5), while the upper limit is less affected. Also note that for an unlocked planet, both limits for $\omega_{i}$ given in Table 3.2 were considered.

Eq. (3.1) shows that for tidally locked planets both the semi-major axis of the planets and the mass of the host star will influence $\omega$ and thus the resulting magnetic moment $\mathcal{M}$. The dependency of maximum and minimum magnetic moment on the orbital distance is 


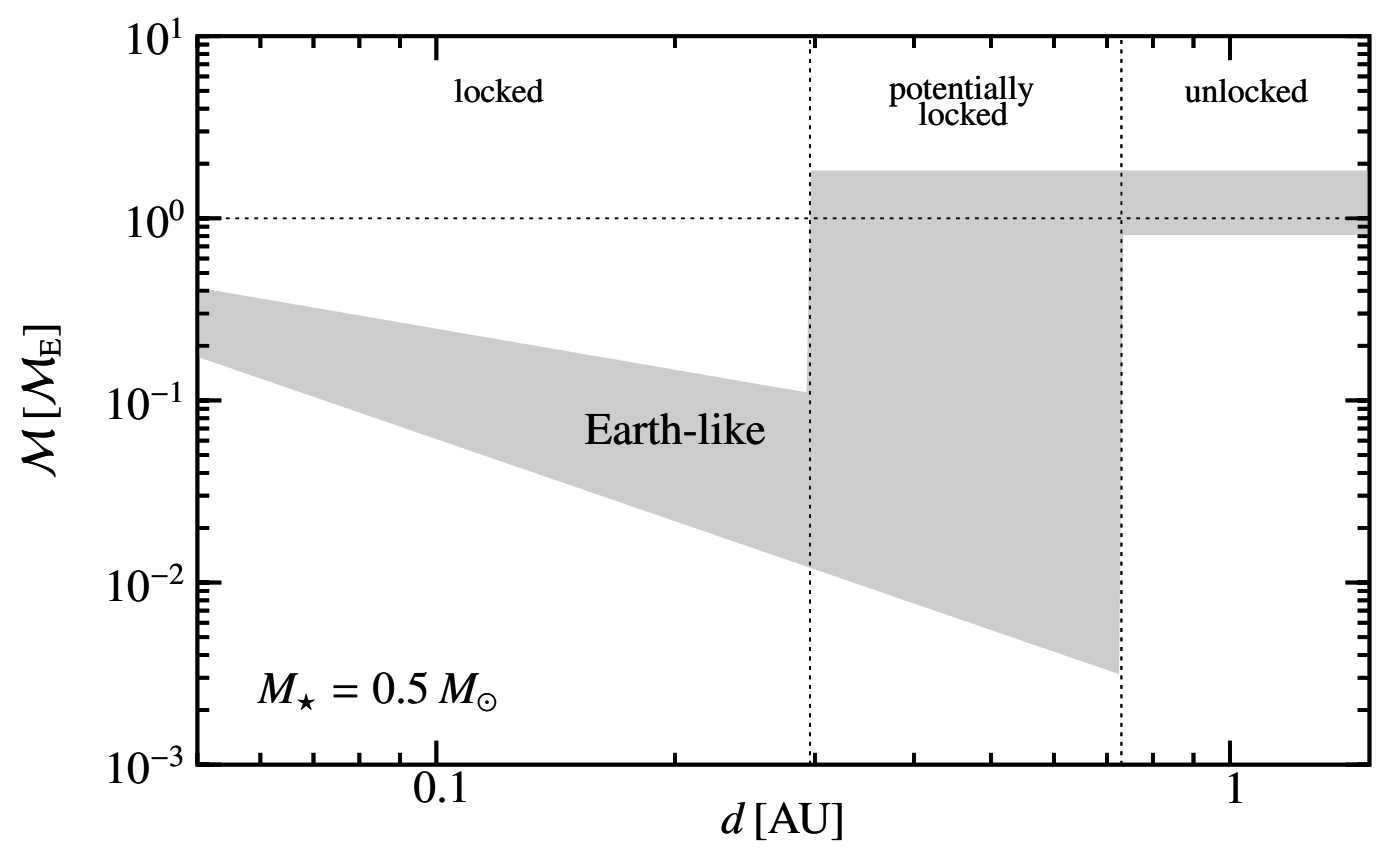

Figure 4.8: Estimated magnetic moment for an Earth-like planet in orbit around a star with mass $M_{\star}=0.5 M_{\odot}$ as a function of orbital distance. The magnetic moment is given in units of Earth's current magnetic moment.

shown in Fig. 4.8. The area between maximum and minimum moment is shaded in grey. For large orbital distances, where the tidal locking timescale is very long (i.e. $\tau_{\text {sync }}>10$ Gyr), the planetary rotation rate is taken to be constant. In this case, both limits for $\omega_{i}$ given in Table 3.2 were considered. The magnetic moment is then given by $\mathcal{M}_{\text {rr }}$, i.e. the right column of Table 4.7. For low rotation rates (corresponding to $\tau_{\text {sync }}<0.1 \mathrm{Gyr}$ ), tidal locking sets in and $\omega$ is given by equation (3.1), leading to a dependency on the semi-major axis. For an orbital distance of $d=0.2 \mathrm{AU}$, the value of $\mathcal{M}$ corresponds to the value given in the left column of Table 4.7. For intermediate distances, it is not sure whether an observed planet will be subject to tidal locking (i.e. $0.1 \mathrm{Gyr}<\tau_{\text {sync }}<10 \mathrm{Gyr}$ ) and both cases have to be considered. In Fig. 4.8, the lower limit of the initial rotation rate $\omega_{\mathrm{i}}$ defines the upper boundary of the "potentially locked" regime as well as the lower limit for the magnetic moment in the "unlocked" regime. Note that the magnetic moment of the Earth is close to the lower limit of the expected magnetic moment range. This is the case because a considerable portion of the angular momentum of the Earth-Moon system resides in the Moon's orbital motion rather than in the Earth's rotation.

Similarly to Figure 4.8, Figures 4.9, 4.10 and 4.11 show the magnetic moment $\mathcal{M}$ as a function of the orbital distance $d$ for the cases of a Mercury-like planet, a "Large Earth" and an "Ocean Planet", respectively. In all three cases, a stellar mass of $0.5 M_{\odot}$ is assumed. The comparison confirms the results already contained in Table 4.7, e.g. the very small magnetic moment of a Mercury-like planet, or the relatively large magnetic moment of a large terrestrial planet (the "Large Earth" case).

As was discussed in Section 3.1.2, not all close-in terrestrial exoplanets should be expected to be exactly tidally locked. The reason is that thermal atmospheric tides generated 


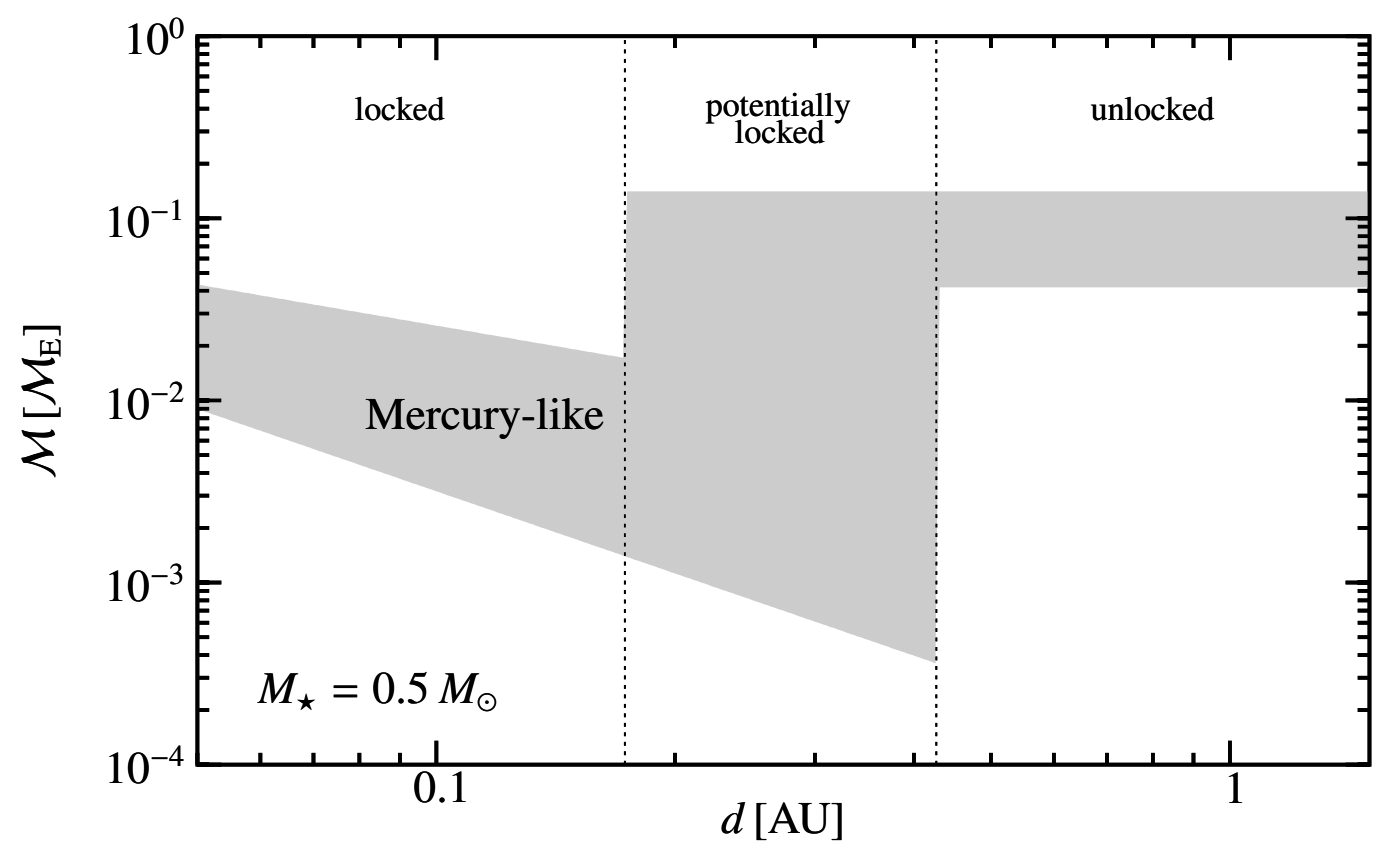

Figure 4.9: Estimated magnetic moment for a Mercury-like planet orbiting a star with mass $M_{\star}=0.5 M_{\odot}$ as a function of orbital distance. The magnetic moment is given in units of Earth's current magnetic moment.

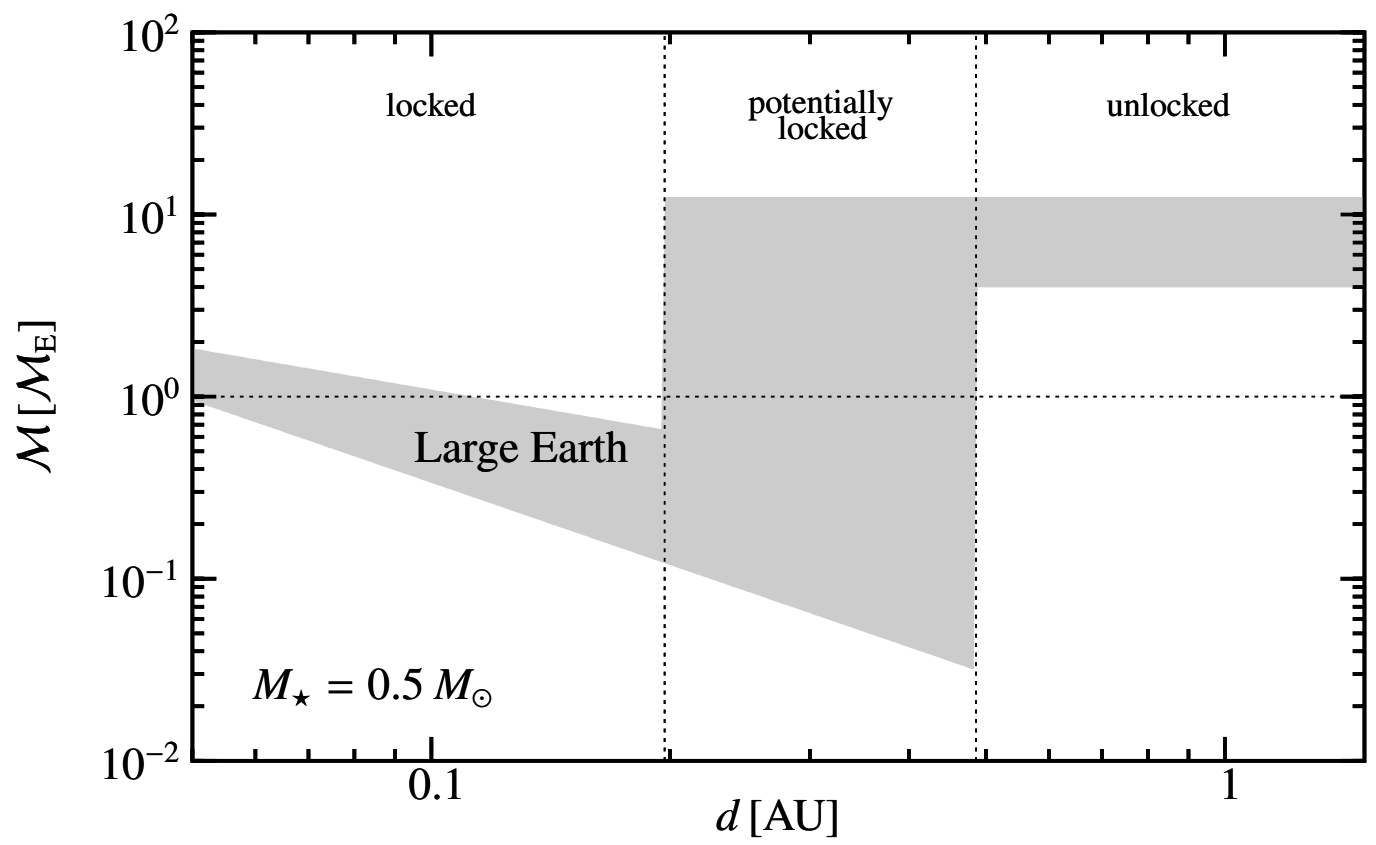

Figure 4.10: Estimated magnetic moment for a "Large Earth" orbiting a star with mass $M_{\star}=0.5 M_{\odot}$ as a function of orbital distance. The magnetic moment is given in units of Earth's current magnetic moment. 


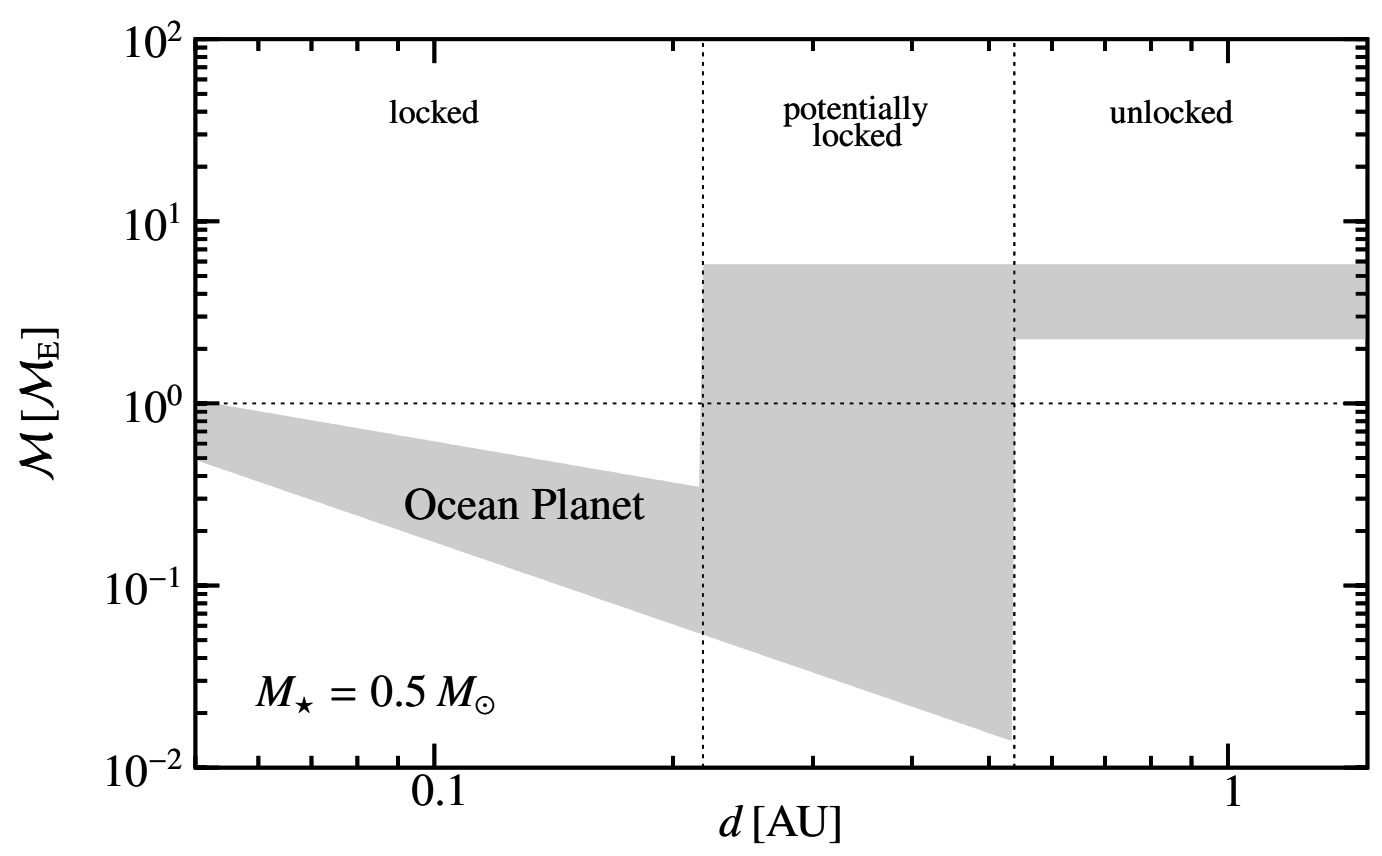

Figure 4.11: Estimated magnetic moment for an "Ocean Planet" orbiting a star with mass $M_{\star}=0.5 M_{\odot}$ as a function of orbital distance. The magnetic moment is given in units of Earth's current magnetic moment.

from the solar heating of the atmosphere can drive planets away from synchronous rotation (Correia et al. 2003). This is especially true for planets with a dense atmosphere (like Venus). For this reason, the effect of doubling the planetary rotation rate $\omega$ is studied. As shown in the middle column of Table $4.7\left(\mathcal{M}_{\mathrm{dr}}\right)$, the lower limit for the magnetic moment is doubled, but the influence on the upper limit of $\mathcal{M}$ is weak. When applying the magnetic moment calculations in Chapter 7, only the upper limit for the magnetic moment will be considered. For this reason, this effect can be neglected in a first approximation.

The smallness of the planetary magnetic moments has important consequences for the sizes of the planetary magnetospheres. This issue will be discussed in Section 5.3.4. 


\title{
5 Formation of magnetospheres by stellar winds
}

\author{
Hui!-Wie saust der Wind!-Johohe! Hojohe! \\ Richard Wagner, Der fliegende Holländer (1841)
}

Knowing the magnetic moment, one can proceed to examine the interaction between the planetary magnetic field and the stellar wind. For this purpose, it is necessary to obtain information on the stellar wind properties. The stellar wind model employed in this work is presented in Section 5.1. For close-in planets, stellar CMEs constitute an important component of the planetary environment. This is discussed in Section 5.2. The magnetosphere, which is shaped by the interaction of stellar wind and stellar CMEs with the planetary magnetic field, is described in Section 5.3 .

\subsection{Stellar winds}

Not only the planetary rotation rate (as discussed in Section 3.1) and therefore the size of the magnetic moment (as discussed in Section 4) are very different for a planet in close orbit when compared to a planet at a larger orbital distance, but also the stellar wind is much denser because of the close proximity of the star. In Section 5.3.2 it will be shown how this contributes to a further reduction of the size of the planetary magnetosphere.

The solar wind is variable on short timescales. For example, the solar wind is known to exist in two main "states", namely the slow solar wind and high-speed streams. The situation is further complicated by the existence of regions where these two types of solar wind interact, and by a high variability of the slow solar wind. To get a simple model of the interaction of the stellar wind with a planetary magnetosphere, the short-time variations of the stellar wind are neglected, and a stationary stellar wind model is used to obtain the average stellar wind conditions.

As far as the radial dependence of the stellar wind density and velocity is concerned, the isothermal stellar wind model of Parker (1958) will be used throughout this work. This stellar wind model is described in Section 5.1.1.1. The differences of the stellar wind model by Weber and Davis (1967) will be briefly discussed in Section 5.1.1.2, showing the Parker model to be sufficient as a first approximation. When planets around stars of different ages are discussed, it is necessary to take into account the evolution of the average stellar wind parameters on long time-scales. This subject is presented in Section 5.1.2. The influence of the planetary orbital velocity on the effective velocity of the stellar wind is studied in Section 5.1.3. Finally, in Section 5.1.4 the procedure is explained with 
which the stellar wind parameters velocity and density are estimated for exoplanetary host stars. Numerical values for these parameters are given for different situations.

\subsubsection{Radial dependence}

It is important to note that close to the central star, the stellar wind density does not simply quadratically decrease with distance (i.e. $n(d) \not \subset d^{-2}$ ). The reason is that, at these distances, the stellar wind has not yet reached the quasi-asymptotic velocity regime (Preusse et al. 2005, Preusse 2006). Due to the conservation of mass, the integrated mass flux through a sphere with radius $d$ around the star, $\dot{M}_{\star}=4 \pi d^{2} n v m$, where $m$ is the mass of the stellar wind protons, has to be constant. Thus, at distances where the stellar wind velocity is increasing with distance, the density decrease has to be enhanced accordingly. For these reasons, a model with a radially dependent stellar wind velocity is required to correctly describe the interaction of close-in exoplanets with their surroundings.

\subsubsection{Stellar wind model of Parker}

Throughout this work, the stellar wind will be described by the solution of the hydrodynamic, isothermal model of Parker (1958). In accordance with the observations, this model describes a solar wind with low velocity and large acceleration near the Sun, whereas at larger distances the velocity is large and the acceleration strongly decreases, as can be seen in Fig. 5.1(a) In the Parker model, the interplay between stellar gravitation and pressure gradients leads to a supersonic gas flow for sufficiently large substellar distances $d$ (i.e. beyond the critical radius defined below).

From the Parker model, the radial dependence of the stellar wind density and velocity can be determined, for example, for planet-hosting stars. The derivation of Parker's wind equation, eq. (5.1), can be found in the original work by Parker (1958), in more recent research work (e.g. Mann et al.|1999, Stracke 2004, Preusse 2006) or in various textbooks (e.g. Brandt 1970, Prölss 2001). It is usually written in the form

$$
\left(\frac{v(d)}{v_{\text {crit }}}\right)^{2}-2 \ln \left(\frac{v(d)}{v_{\text {crit }}}\right)=4 \ln \frac{d}{r_{\text {crit }}}+4 \frac{r_{\text {crit }}}{d}-3 .
$$

The velocity $v(d)$ at the distance $d$ from the star is determined by the solution of eq. (5.1) which passes through $v_{\text {crit }}$ for $r_{\text {crit }}$ (i.e. with $\left.v\left(d=r_{\text {crit }}\right)=v_{\text {crit }}\right)$. Here, $v_{\text {crit }}$ denotes the critical velocity defined by

$$
v_{\text {crit }}=\sqrt{\frac{k_{\mathrm{B}} T}{m}}
$$

and $r_{\text {crit }}$ is the critical radius given by

$$
r_{\text {crit }}=\frac{m G M_{\star}}{4 k_{\mathrm{B}} T} .
$$

Furthermore, $k_{\mathrm{B}}$ denotes Boltzmann's constant, $T$ is the temperature of the stellar wind (which is constant and supposed to be identical to the corona temperature $T_{\text {corona }}$ in this

\footnotetext{
${ }^{1}$ Note that for large distances the assumption of an isothermal heating of the wind is not realistic, and an adiabatic treatment would be more appropriate. However, for the planetary distances discussed in this work, the error introduced by assuming isothermal conditions is small enough to justify this treatment.
} 
isothermal model), $m$ is the mass of the stellar wind protons, and $G$ is the constant of gravitation.

For a given stellar mass, the velocity profile is determined by the choice of the coronal temperature $T_{\text {corona }}$. Because the stellar mass loss rate $\dot{M}_{\star}=4 \pi d^{2} n v m$ is a free parameter, the density profile $n(d)$ can be obtained from the conservation of mass once the velocity profile $v(d)$ is known:

$$
n(r)=\frac{\dot{M}_{\star}}{4 \pi d^{2} v(d) m} .
$$

The only unknown quantity in this stellar wind model (besides the stellar mass loss rate $\dot{M}_{\star}$, for which the solar value can be taken for the moment) is therefore the temperature of the corona, $T_{\text {corona. }}$. For the solar system, it can be found by requiring the solution to reproduce the solar wind velocity and density near Earth. With $v=425 \mathrm{~km} / \mathrm{s}$ and $n=6.59 \cdot 10^{6} \mathrm{~m}^{-3}$ at $d=1 \mathrm{AU}$ (Schwenn 1990, Mann et al. 1999), one finds $T_{\text {corona }}=0.81 \mathrm{MK}$ and $\dot{M}_{\star}=m \cdot 7.88 \cdot 10^{35} \mathrm{~s}^{-1}$, which allow the determination of $v(d)$ and $n(d)$. Both profiles are show in Fig. 5.1. In Fig. 5.1(a), the strong acceleration of the stellar wind for small distances (including typical orbital distances of extrasolar planets) is visible. Fig. 5.1(b) shows that, correspondingly, the density deviates from a simple $d^{-2}$ power law for small distances. Similarly (but taking into account contributions from heavier constituents in the solar wind), Mann et al. (1999) found that for a coronal temperature of $1 \mathrm{MK}$, a heliospheric density model follows which is consistent with particle densities measured by the spacecraft Helios 1, Helios 2 and Ulysses.

This stellar wind model will be applied to exoplanet host stars in Section 5.1.4. Before this can be done, however, the influence of the stellar age on the stellar wind velocity and density has to be analysed. This is done in Section 5.1.2.

\subsubsection{Stellar wind model of Weber and Davis}

Weber and Davis (1967) extended the purely hydrodynamic solar wind model of Parker (1958) to a magneto-hydrodynamic model by including the Lorentz force caused by the solar magnetic field. Also, in the Weber and Davis (1967) model, the rotation of the star is taken into account, causing the velocity and the magnetic field to have both azimuthal and radial components.

The Parker (1958) model represents the limit of vanishing stellar rotation and vanishing stellar magnetic field of the Weber and Davis (1967) model. The Sun, for example, is rotating slowly enough so that the solar wind model of Parker (1958) is sufficient to describe the solar wind. For stars with higher rotation rates, however, the influence of rotation and stellar magnetic field leads to a higher stellar wind velocity than the stellar wind model of Parker. Preusse et al. (2005, Fig. 2) show that for a star with a rotation period of 30 days, the results of the two stellar wind models are indistinguishable. On the other hand, for rotation periods of 3 days, the difference in stellar wind velocity $v$ can reach a factor of two in certain cases. For a fixed stellar wind flux, the Weber and Davis (1967) solution will also yield a higher stellar wind ram pressure $n v^{2}$, and thus smaller planetary magnetospheres.

As will be discussed in Section 5.1.2, the stellar rotation rate monotonically decreases with increasing stellar age. For this reason, the Parker stellar wind model is sufficient for old stars (like the Sun), and the Weber and Davis stellar wind model is more accurate 


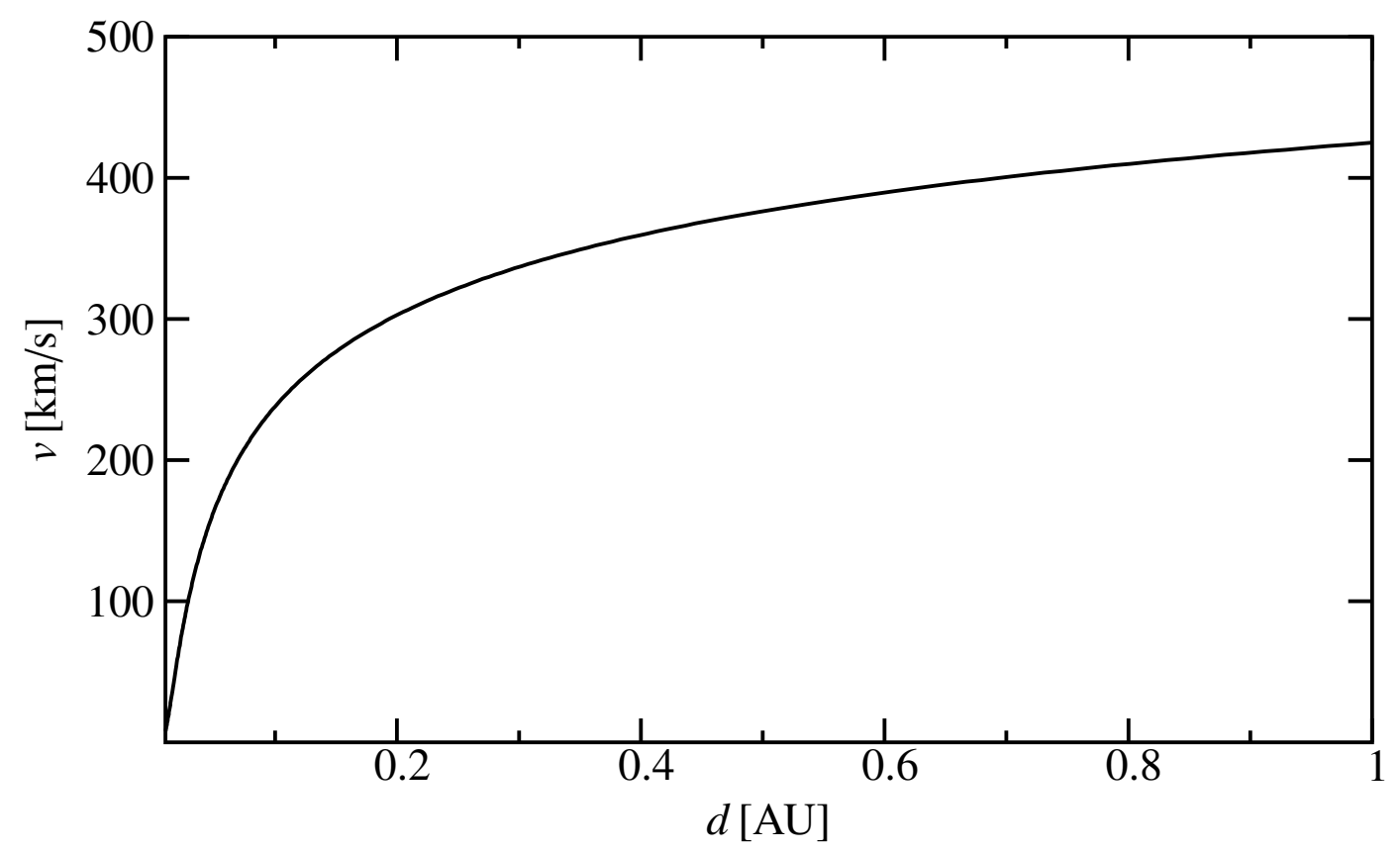

(a) Solar wind velocity

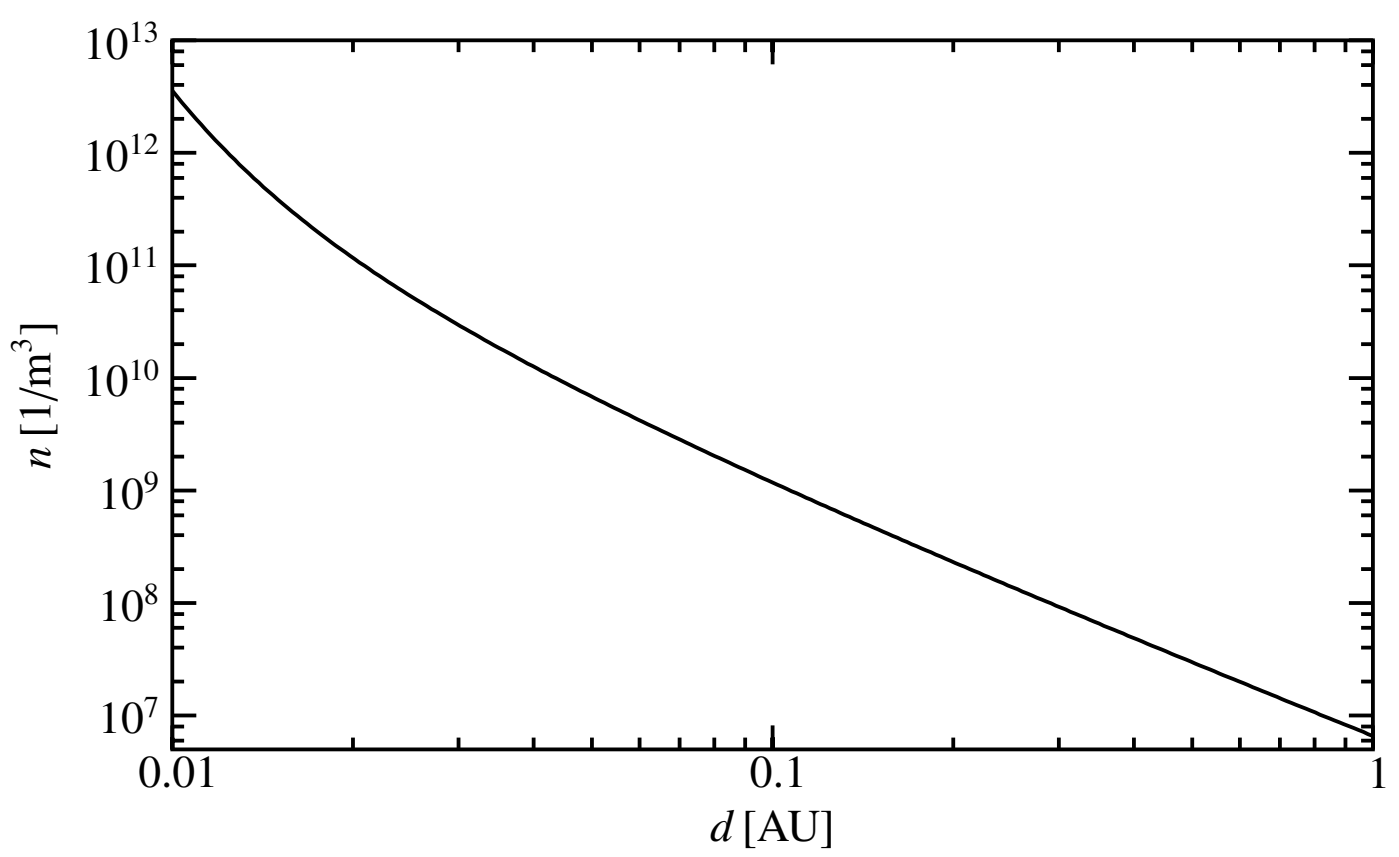

(b) Solar wind density

Figure 5.1: Solar wind velocity and solar wind density according to the Parker model. The corona temperature and total flux are chosen such that at $1 \mathrm{AU}, v=425 \mathrm{~km} / \mathrm{s}$ and $n=6.59 \cdot 10^{6} \mathrm{~m}^{-3}$.

for young stars. The difference between the models also depends on the stellar mass and radius. The largest and heaviest star considered in this work is $\tau$ Bootes. For this star, 
Grießmeier et al. (2006c) found that the difference between the stellar wind solutions of Parker (1958) and Weber and Davis (1967) is negligible (i.e. below 5\%) for stellar rotation periods $P_{\text {rot }}>5$ days. This result was obtained for the case of a strong stellar magnetic field. For a weaker field, the difference is even less. Taking the solar values as $t_{\odot}=4.6$ Gyr and $P_{\text {rot, } \odot}=25.5$ days, one can obtain the stellar age corresponding to such a rotation period from eq. (5.9). It is found that stellar rotation periods are larger than 5 days for stellar ages of $t>0.45 \mathrm{Gyr}$. Current estimations of the stellar wind properties, however, are only valid for stellar ages $\geq 0.7 \mathrm{Gyr}$ (Wood et al.2005a). For this reason, only stellar ages of at least $0.7 \mathrm{Gyr}$ are considered within this work, which corresponds to rotation periods $\geq 7.0$ days. Thus, the stellar wind model of Parker (1958) is sufficient, and the model of Weber and Davis (1967) is not required.

Much more information on the Weber and Davis stellar wind model and its application to exoplanet host stars can be found in Preusse et al. (2005) and Preusse (2006). Alternative stellar wind models are discussed, for example, in Brandt (1970).

\subsubsection{Long term time dependence}

As already mentioned in Section 5.1, the goal of this section is not to discuss the shortterm variability of stellar wind parameters, but their evolution on astronomical timescales. The evolution of the stellar wind velocity $v$ and density $n$ of solar-like stars is estimated using the scaling for stellar mass loss provided by Wood et al. (2002) as well as the scaling for the velocity obtained by Newkirk (1980). The discussion of this section closely follows the work of Grießmeier et al. (2004) and Lammer et al. (2004), including some more recent results.

For a long time, stellar winds of solar-like stars were not accessible to observations. On the other hand, the existence of such winds was expected for stars having a hot corona like the Sun. Observations with X-ray satellites like Einstein and ROSAT made it clear that hot coronae are a typical property of cool main sequence stars. For this reason, solar-like winds can be expected for all Sun-like stars. The detection of such winds, however, turned out to be difficult. Different attempts to detect such winds directly were unsuccessful (Wood 2004). Finally, they were detected indirectly through their interaction with the partially ionised local interstellar medium (LISM). In the region between the astropause and the astrospheric bow shock, the LISM is heated and compressed. Through charge exchange, a population of neutral hydrogen atoms with high temperature is created. The characteristic Ly $\alpha$ absorption (at $1216 \AA$ ) of this population was $\mathrm{s}^{2}$ detectable with the high-resolution observations of the GHRS and STIS spectrometers of the Hubble Space Telescope (HST). The amount of absorption depends on the size of the astrosphere, which is a function of the properties of the LISM and of the stellar wind ram pressure $\left(n v^{2}\right)$. Comparing the measured absorption to that calculated by hydrodynamic codes, these measurements allowed the first empirical estimation of stellar mass loss rates. By using data obtained for stars with different X-ray fluxes (used as a proxy for the stellar age), it was possible to determine the evolution of the stellar mass loss rate as a function of stellar age (Wood et al. 2002, Wood 2004, Wood et al.2005a). The original derivation of this dependency was based on the observation of only a few $\mathrm{G}$ and $\mathrm{K}$ stars, plus one

\footnotetext{
2 The breakdown of the STIS instrument on board HST in 2004 means that no further Ly $\alpha$ spectra will be available in the forseeable future (Wood et al. 2005b).
} 
M star (Wood et al. 2002). By analysing additional data of the HST archives (Wood et al. 2005b), it was later possible to confirm this relation, however with two restrictions: (a) it appears not to be applicable to evolved stars, but only to main sequence stars, and (b) it seems not to be valid for stellar ages below $0.7 \mathrm{Gyr}$ (Wood et al.2005a). In the following, this relation will be used to obtain an estimation for the time evolution of the stellar wind parameters velocity and density.

Wood et al. (2002) found that the stellar mass loss rate $\dot{M}_{\star}$ estimated from the astrospheric absorption is related to the observed X-ray flux $\phi_{\mathrm{X}}$ of solar-like stars. When the coronal activity is at its maximum, this relation can be written as a power law (Wood et al. 2002, Wood 2004):

$$
\dot{M}_{\star} \propto \phi_{\mathrm{X}}^{1.15} .
$$

The X-ray flux of a cool main sequence Sun-like star depends on the stellar rotation period $P_{\text {rot }}$, which is in turn correlated with the stellar age. According to Wood et al. (2002), these relations can be expressed as

$$
\phi_{\mathrm{X}} \propto P_{\text {rot }}^{-2.9}
$$

and

$$
P_{\text {rot }} \propto t^{0.6}
$$

respectively. In these relations $t$ denotes the time elapsed since the formation of the stellar system. For the present day solar system, $t=4.6$ Gyr. From eqs. (5.5) and (5.6), one obtains a power law relationship for the mass loss rate as a function of rotation period, $\dot{M}_{\star}\left(P_{\text {rot }}\right)$. At this point, a complication occurs. Eq. 5.5) does look like a scaling law for the mass loss, but what is really measured by Wood et al. (2002, 2005a) is the total ram pressure, i.e. the product of mass loss and solar wind velocity. The mass loss given by Wood et al. (2002) was obtained by assuming a constant velocity $v=400 \mathrm{~km} / \mathrm{s}$. As scaling relations for both $n$ and $v$ are sought, one has to correct for this problem by writing $\dot{M}_{\star} v$ rather than $\dot{M}_{\star}$ in eq. 5.5 . Thus:

$$
\dot{M}_{\star} v \propto P_{\text {rot }}^{-3.3} .
$$

To obtain the time dependence, a relation for $P_{\text {rot }}(t)$ is substituted into eq. 5.8. In principle, eq. 5.7) could be used, but for consistency with the velocity scaling law given below, the scaling relation derived by Newkirk (1980) is taken:

$$
P_{\text {rot }} \propto\left(1+\frac{t}{\tau}\right)^{0.7}
$$

with the time constant $\tau=2.56 \cdot 10^{7} \mathrm{yr}$ calculated according to Newkirk (1980). Combining eqs. (5.8) and (5.9), it is possible to derive a power law for the stellar mass loss:

$$
\dot{M}_{\star} v \propto\left(1+\frac{t}{\tau}\right)^{-2.3}
$$

On the other hand, the stellar mass loss depends linearly on $v$ and $n$ :

$$
\dot{M}_{\star}=4 \pi R_{\star}^{2} n\left(d=R_{\star}\right) v\left(d=R_{\star}\right) m=4 \pi d^{2} n v m,
$$

where $R_{\star}$ is the stellar radius, $d$ is the substellar distance, and $m$ is the mass of the solar wind protons. Hence, by finding an independent scaling relation for the stellar wind 
velocity $v$, the stellar wind density $n$ directly follows from eq. (5.10). The time-dependent behaviour of the solar wind velocity can be taken as (Newkirk 1980, Zhang et al. 1993)

$$
v(t)=v_{0}\left(1+\frac{t}{\tau}\right)^{-0.4}
$$

Note that the argumentation of Newkirk (1980) would lead to a different stellar wind velocity $v(t)$ if a different relation for $P_{\text {rot }}(t)$, such as eq. (5.7), was used. Using the mass loss relation, eq. (5.10), and combining it with eqs. (5.11) and (5.12), the particle density can be determined to be

$$
n(t)=n_{0}\left(1+\frac{t}{\tau}\right)^{-1.5} .
$$

The proportionality constants are determined by the present-day conditions. In accordance with Mann et al. (1999) and Preusse (2006), the current stellar wind parameters are taken from the long-term averages of Schwenn (1990), with $v=425 \mathrm{~km} / \mathrm{s}$ and $n=6.59 \cdot 10^{6} \mathrm{~m}^{-3}$ for $t=4.6 \mathrm{Gyr}$ and at $d=1 \mathrm{AU}$. This results in $v_{0}=3397 \mathrm{~km} / \mathrm{s}$ and $n_{0}=1.6 \cdot 10^{10} 1 / \mathrm{m}^{3}$ for a distance of $d=1 \mathrm{AU}$. The time constant is $\tau=2.56 \cdot 10^{7} \mathrm{yr}$ (calculated from Newkirk 1980). As noted before, eqs. (5.12) and (5.13) are probably not applicable for stellar ages $t \lesssim 0.7$ Gyr. For distances other than 1 AU, $v(d)$ and $n(d)$ have to be calculated according to Section 5.1.1.1, choosing $T_{\text {corona }}$ and $\dot{M}_{\star}$ such that at $1 \mathrm{AU}$ both $v$ and $n$ are consistent with the results from eqs. (5.12) and (5.13).

The time variation of $v$ and $n$ at a distance of 1 AU from the Sun are shown in Fig. 5.2. One can clearly see, that the stellar wind velocity of a young star (1 Gyr after reaching the main sequence) is about twice as high as the velocity of the present day solar wind (at 4.6 Gyr). Likewise, the density of the stellar wind of a young star is increased by more than one order of magnitude.

Obviously, for young stellar systems, the stellar wind ram pressure $n v^{2}$ is therefore much larger than for a star of the age of our solar system. In the following, three typical cases will be treated: (a) the stellar wind of a very young star of 0.7 Gyr age, which is the lowest age for which eqs. (5.12) and (5.13) are valid, (b) the stellar wind of a young star of $1.0 \mathrm{Gyr}$ age, which corresponds to the estimated age of $\tau$ Bootes (see Table 2.2), and (c) the stellar wind of a star with the age of the Sun $(4.6 \mathrm{Gyr})$. For these cases, the stellar wind parameters will be given in Section 5.1.4. The resulting compression of planetary magnetospheres will be discussed in Sections 5.3.3 and 5.3.4.

\subsubsection{Influence of the orbital velocity}

For effects concerning the interaction between the stellar wind and a planetary magnetopause, the relevant quantity is not the stellar wind velocity relative to its star (as used in the previous sections), but the stellar wind velocity relative to the planet. Especially for close-in planets the orbital velocity may be of the order of the stellar wind velocity, or even larger (Stracke 2004, Fig. 3.7).

For small orbital distances, where this effect becomes important, tidal circularisation of the planetary orbit sets in (see Section 3.2). For circular orbits, the orbital velocity $v_{\text {orbit }}$ 


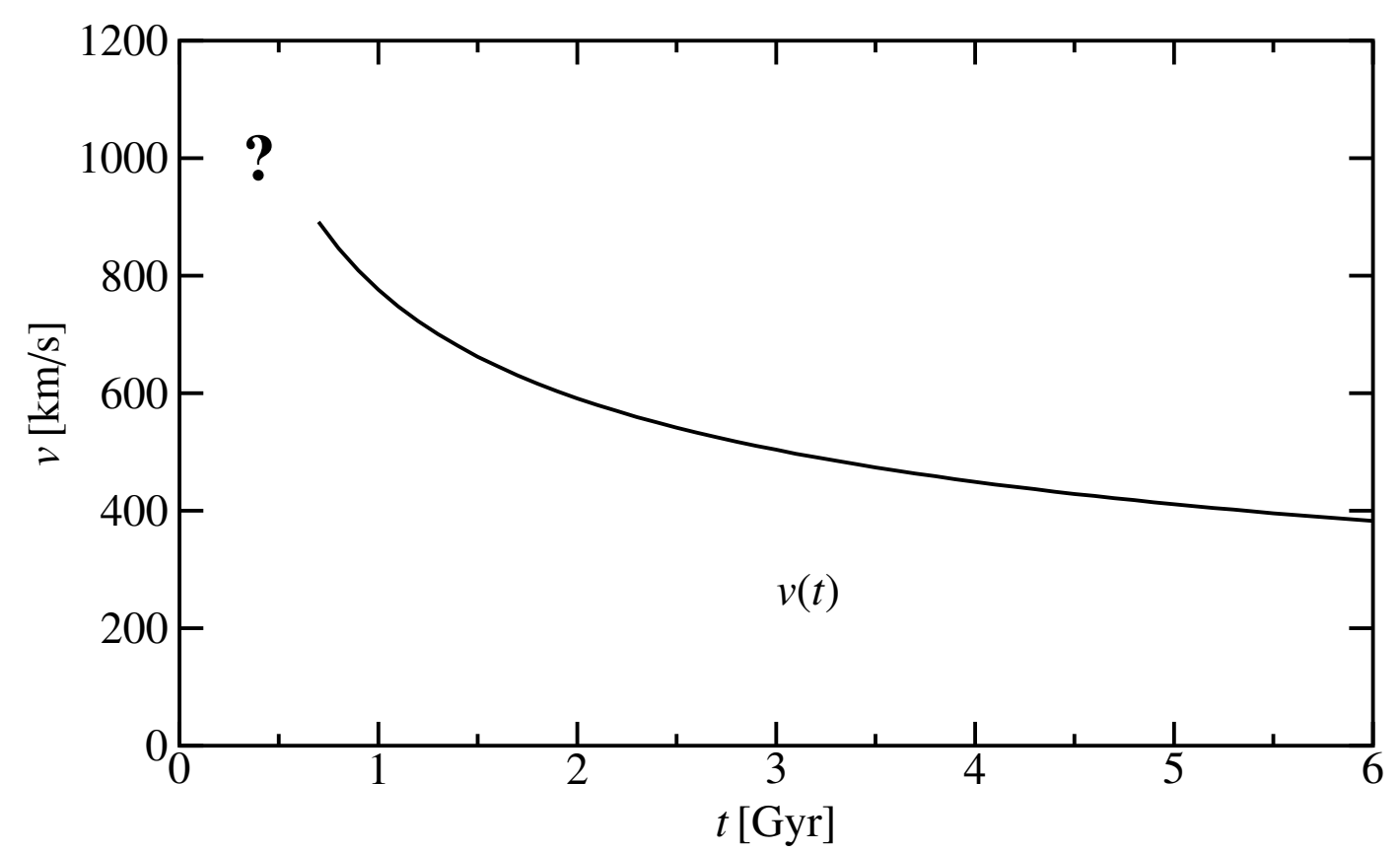

(a) Time dependence of solar wind velocity.

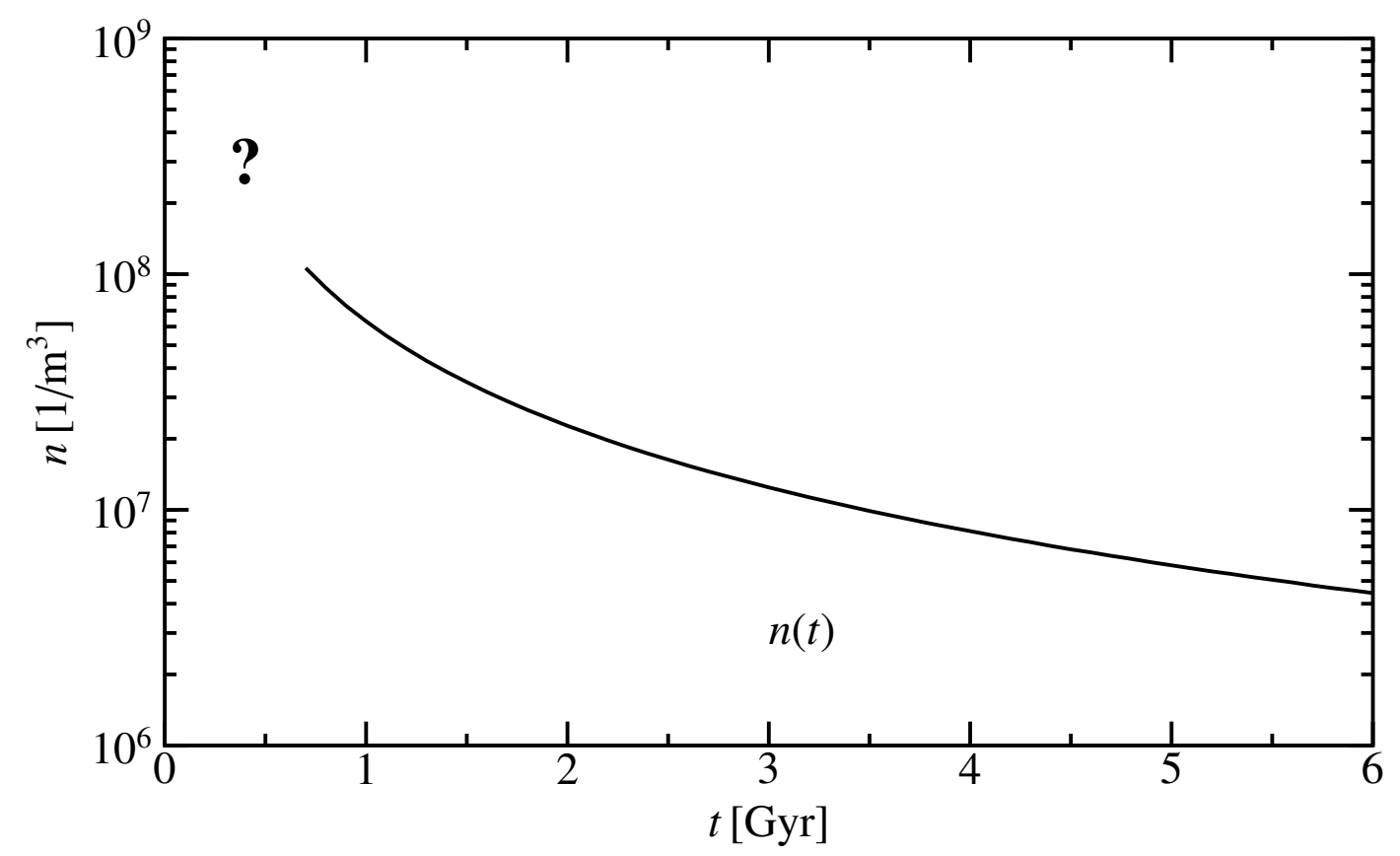

(b) Time dependence of solar wind density.

Figure 5.2: Time evolution of the stellar wind velocity and density at a distance of $1 \mathrm{AU}$ around a Sun-like star.

is perpendicular to the stellar wind velocity $v$, and is given by

$$
v_{\text {orbit }}=\omega_{\text {orbit }} d=\sqrt{\frac{M_{\star} G}{d}},
$$


where eq. 3.1 was inserted for $\omega_{\text {orbit }}$. Then the pressure equilibrium is determined by the effective velocity $v_{\text {eff }}$ defined as:

$$
v_{\text {eff }}=\sqrt{v_{\text {orbit }}^{2}+v^{2}}
$$

At the same time, this effect introduces an angle between the apparent flow direction of the stellar wind and the line connecting the star and the planet.

The difference between $v$ and $v_{\text {eff }}$ is given in Tables 5.1 and 5.2 for some typical cases. In Section 5.3.2, the size of the magnetosphere will be calculated with this effective velocity $v_{\text {eff }}$ rather than the stellar wind velocity $v$.

\subsubsection{Resulting stellar wind parameters}

In this section, the procedure for the evaluation of the stellar wind parameters for different configurations is described. Also, some typical values are given.

The procedure (first applied in Grießmeier et al.2005b) to obtain the stellar wind velocity $v_{\text {eff }}\left(d, t, M_{\star}, R_{\star}\right)$ and density $n\left(d, t, M_{\star}, R_{\star}\right)$ at the location of an exoplanet (i.e. at distance $d$ ) for a host star of given age $t$, mass $M_{\star}$, and radius $R_{\star}$ consists of the following:

1. For a prescribed stellar age, the corresponding values of the solar wind velocity $v\left(t, 1 \mathrm{AU}, M_{\odot}, R_{\odot}\right)$ and solar wind density $n\left(t, 1 \mathrm{AU}, M_{\odot}, R_{\odot}\right)$ at $1 \mathrm{AU}$ are obtained from eqs. (5.12) and (5.13), respectively. The details of this calculation are given in Section 5.1.2. From these quantities, the solar mass loss $\dot{M}_{\odot}$ is calculated according to eq. (5.11):

$$
\dot{M}_{\odot}(t)=4 \pi d_{0}^{2} n\left(d_{0}\right) v\left(d_{0}\right) m
$$

where $d_{0}=1 \mathrm{AU}$.

2. Eq. (5.11) shows that the stellar mass loss rate (and thus the stellar wind density) are proportional to the stellar surface. Thus, the stellar mass loss has to be calculated from the solar mass loss obtained in step 1 by

$$
\dot{M}_{\star}(t)=\dot{M}_{\odot}(t)\left(\frac{R_{\star}}{R_{\odot}}\right)^{2} .
$$

If the mass of the star is given, but its radius is unknown (e.g. for the typical test case of a star of 0.5 solar masses), then the stellar radius has to be obtained first. In such a case, the stellar radius is taken to be the Zero-Age Main-Sequence (ZAMS) radius for a star of solar metallicity, calculated with the equations provided by Tout et al. (1996). In the case of K and M stars, where the temporal evolution of the stellar radius is negligible for stellar ages of the order of 1 Gyr (Chabrier and Baraffe 1997, Figure 7), the ZAMS radius of the star yields an excellent estimation for its true radius. In the case of $\mathrm{G}$ stars, observed radii of planetary host stars will be taken instead. Once $R_{\star}$ is known, $\dot{M}_{\star}(t)$ is calculated with eq. (5.17).

3. Rather than assume a stellar wind velocity $v$ independent of $d$, and a stellar wind density $n$ that quadratically decreases with distance (i.e. $n(d) \propto d^{-2}$ ), as was done 
5 Formation of magnetospheres by stellar winds

\begin{tabular}{|l||c|c|c|}
\hline planet around a G star & $4.6 \mathrm{Gyr}$ & $1.0 \mathrm{Gyr}$ & $0.7 \mathrm{Gyr}$ \\
\hline$v(t)$ (velocity at $0.05 \mathrm{AU})[\mathrm{km} / \mathrm{s}]$ & 164 & 421 & 509 \\
$v_{\text {eff }}(t)$ (effective velocity at $\left.0.05 \mathrm{AU}\right)[\mathrm{km} / \mathrm{s}]$ & 212 & 442 & 526 \\
$n(t)$ (density at $0.05 \mathrm{AU})\left[\mathrm{m}^{-3}\right]$ & $6.82 \cdot 10^{9}$ & $4.65 \cdot 10^{10}$ & $7.43 \cdot 10^{10}$ \\
\hline$v(t)($ velocity at $0.2 \mathrm{AU})[\mathrm{km} / \mathrm{s}]$ & 303 & 607 & 709 \\
$v_{\text {eff }}(t)$ (effective velocity at $\left.0.2 \mathrm{AU}\right)[\mathrm{km} / \mathrm{s}]$ & 310 & 611 & 712 \\
$n(t)($ density at $0.2 \mathrm{AU})\left[\mathrm{m}^{-3}\right]$ & $2.31 \cdot 10^{8}$ & $2.02 \cdot 10^{9}$ & $3.34 \cdot 10^{9}$ \\
\hline$v(t)($ velocity at $1.0 \mathrm{AU})[\mathrm{km} / \mathrm{s}]$ & 425 & 776 & 891 \\
$v_{\text {eff }}(t)$ (effective velocity at $\left.1.0 \mathrm{AU}\right)[\mathrm{km} / \mathrm{s}]$ & 426 & 777 & 892 \\
$n(t)$ (density at $1.0 \mathrm{AU})\left[\mathrm{m}^{-3}\right]$ & $6.59 \cdot 10^{6}$ & $6.31 \cdot 10^{7}$ & $1.06 \cdot 10^{8}$ \\
\hline$T_{\text {corona }}$ (corona temperature) $[\mathrm{MK}]$ & 0.81 & 2.09 & 2.61 \\
\hline
\end{tabular}

Table 5.1: Stellar wind parameters for distances of $0.05 \mathrm{AU}, 0.2 \mathrm{AU}$ and $1.0 \mathrm{AU}$ around a Sun-like G type star $\left(M_{\star}=M_{\odot}\right.$, and radius $\left.R_{\star}=R_{\odot}\right)$.

before (e.g. Grießmeier et al.|2004, 2005a), a Parker-like stellar wind model is used to find $n(d)$ and $v(d)$ as a function of the distance to the star. As described in Section 5.1.1.1, the corona temperature $T_{\text {corona }}$ is adjusted until the stellar wind velocity ${ }^{3}$ at $1 \mathrm{AU}$ corresponds to the value which was obtained in step 1 . With this value of $T_{\text {corona }}, v$ can be determined for any value of $d$ by solving eq. (5.1). Thus, $v(d, t)$ is obtained.

4. The density $n(d, t)$ is then obtained by dividing the stellar mass loss $\dot{M}_{\star}(t)$ obtained in step 2 by $4 \pi d^{2} v(d) m$, where $v(d, t)$ was obtained in step 3 .

5. Finally, the stellar wind velocity $v(d, t)$ (obtained in step 3 ) is replaced by the effective velocity $v_{\text {eff }}$ given by eq. (5.15), as described in Section 5.1.3.

Unfortunately, there is no closed analytical form for the resulting functions $n(d)$ and $v(d)$. Tables 5.1 and 5.2 give the stellar wind parameters resulting from this procedure for a few typical cases. Two different stellar types are considered: Table 5.1 shows stellar wind parameters typical for a Sun-like G star with mass $M_{\star}=M_{\odot}$ and radius $R_{\star}=R_{\odot}$. Results for a smaller $\mathrm{K} / \mathrm{M}$ type star of mass $M_{\star}=0.5 M_{\odot}$ and radius $R_{\star}=0.46 R_{\odot}$ are given in Table 5.2. Note that for the $\mathrm{G}$ star, some change in stellar radius is expected during the stellar evolution (Guinan and Ribas 2002, Figure 1). This effect was neglected in the calculations. For the K/M star, the temporal evolution of the stellar radius is negligible for stellar ages of the order of 1 Gyr (Chabrier and Baraffe 1997, Figure 7).

In Tables 5.1 and 5.2, one can see that in this model, the increased stellar wind velocity of young stars is reflected by an elevated corona temperature $T_{\text {corona }}$. The difference

\footnotetext{
${ }^{3}$ Note that eq. 5.1 has more than one solution, of which only one is physically meaningful. At this point, care must be taken to ensure the correct solution is found, i.e. the solution in which $v(d)$ is monotonically increasing.
} 
5.2 Stellar coronal mass ejections

\begin{tabular}{|l||c|c|c|}
\hline planet around a K/M star & $4.6 \mathrm{Gyr}$ & $1.0 \mathrm{Gyr}$ & $0.7 \mathrm{Gyr}$ \\
\hline$v(t)($ velocity at $0.05 \mathrm{AU})[\mathrm{km} / \mathrm{s}]$ & 206 & 472 & 562 \\
$v_{\text {eff }}(t)$ (effective velocity at $\left.0.05 \mathrm{AU}\right)[\mathrm{km} / \mathrm{s}]$ & 226 & 481 & 569 \\
$n(t)$ (density at $0.05 \mathrm{AU})\left[\mathrm{m}^{-3}\right]$ & $1.15 \cdot 10^{9}$ & $8.74 \cdot 10^{9}$ & $1.42 \cdot 10^{10}$ \\
\hline$v(t)($ velocity at $0.2 \mathrm{AU})[\mathrm{km} / \mathrm{s}]$ & 321 & 630 & 732 \\
$v_{\text {eff }}(t)$ (effective velocity at $\left.0.2 \mathrm{AU}\right)[\mathrm{km} / \mathrm{s}]$ & 325 & 631 & 733 \\
$n(t)($ density at $0.2 \mathrm{AU})\left[\mathrm{m}^{-3}\right]$ & $4.58 \cdot 10^{7}$ & $4.09 \cdot 10^{8}$ & $6.80 \cdot 10^{8}$ \\
\hline$v(t)($ velocity at $1.0 \mathrm{AU})[\mathrm{km} / \mathrm{s}]$ & 425 & 776 & 891 \\
$v_{\text {eff }}(t)$ (effective velocity at $\left.1.0 \mathrm{AU}\right)[\mathrm{km} / \mathrm{s}]$ & 425 & 777 & 892 \\
$n(t)$ (density at $1.0 \mathrm{AU})\left[\mathrm{m}^{-3}\right]$ & $1.39 \cdot 10^{6}$ & $1.33 \cdot 10^{7}$ & $2.23 \cdot 10^{7}$ \\
\hline$T_{\text {corona }}$ (corona temperature) $[\mathrm{MK}]$ & 0.69 & 1.84 & 2.31 \\
\hline
\end{tabular}

Table 5.2: Stellar wind parameters for distances of $0.05 \mathrm{AU}, 0.2 \mathrm{AU}$ and $1.0 \mathrm{AU}$ around a star of mass $M_{\star}=0.5 M_{\odot}$ and radius $R_{\star}=0.46 R_{\odot}$.

between $v$ and $v_{\text {eff }}$ is important for the smallest distance (0.05 AU), but is less significant for larger orbital distances. Because of its smaller surface area, the stellar wind density $n$ is much smaller for the $\mathrm{K}$ than for the $\mathrm{G}$ star, whereas the stellar wind velocity $v$ does not strongly depend on the stellar type.

\subsection{Stellar coronal mass ejections}

Recently, stellar coronal mass ejections similar to solar coronal mass ejections (CMEs) were suggested to have a strong influence on close-in extrasolar planets. Two different effects are currently being discussed: (a) the possible increase of atmospheric loss caused by CMEs (Khodachenko et al. 2006), and (b) the enhancement of planetary radio emission from Hot Jupiters (Grießmeier et al. 2006b).

This section provides the parameters necessary to discuss the effect of a stellar CME on an exoplanetary magnetosphere, and extends the discussion of Section 5.1, which deals with average stellar wind conditions. Parameters typical for CMEs, i.e. enhanced particle velocity and density, will be presented in Section 5.2.1. In Section 5.2.2 the rate of occurrence of CME collisions will be estimated for close-in exoplanets, showing that frequent CME-planet collisions have to be expected. Finally, in Section 5.2.3 the parameters (velocity and density) for a CME situation are compared to the stellar wind parameters obtained in Section 5.1.4.

\subsubsection{Density, velocity and temperature}

In this section, typical properties for coronal mass ejections are presented. Not all CMEs have the same properties. Combining a large set of observations, Khodachenko et al. 
(2006) derive the dependence of the average CME density $n_{\mathrm{CME}}$ and of the average $\mathrm{CME}$ velocity $v_{\mathrm{CME}}$ on the substellar distance $d$.

The current knowledge on CMEs was obtained by studying the Sun. Different observational data on CMEs are available for two distinct spatial domains: (a) the near-Sun region (with $d \lesssim 0.14 \mathrm{AU}$ ), where remote observations were obtained by coronagraphs (i.e. the Large Angle and Spectrometric Coronagraph LASCO on board of ESA's Solar and Heliospheric Observatory SoHO), and (b) a region further out (with $d \gtrsim 0.3 \mathrm{AU}$ ), where data were obtained by in-situ observations from various spacecraft (e.g. Helios). With a large database of observations, statistical studies of solar CMEs have been made possible. Connecting the results of remote estimations of the CME parameters near the Sun with those measured in-situ at larger distances, Khodachenko et al. (2006) give two interpolated limiting cases, denoted as weak and strong CMEs, respectively. These two classes have a different dependence of the (average) density on the distance to the Sun $d$. In the following, these quantities will be labelled $n_{\mathrm{CME}}^{\mathrm{w}}(d)$ and $n_{\mathrm{CME}}^{\mathrm{s}}(d)$, respectively.

For weak CMEs, the density $n_{\mathrm{CME}}^{\mathrm{w}}(d)$ behaves as

$$
n_{\mathrm{CME}}^{\mathrm{w}}(d)=n_{\mathrm{CME}, 0}^{\mathrm{w}}\left(d / d_{0}\right)^{-2.31}
$$

where the density at $d_{0}=1 \mathrm{AU}$ is given by $n_{\mathrm{CME}, 0}^{\mathrm{w}}=n_{\mathrm{CME}}^{\mathrm{w}}\left(d=d_{0}\right)=4.88 \cdot 10^{6} \mathrm{~m}^{-3}$.

For strong CMEs, Khodachenko et al. (2006) find

$$
n_{\mathrm{CME}}^{\mathrm{s}}(d)=n_{\mathrm{CME}, 0}^{\mathrm{s}}\left(d / d_{0}\right)^{-2.99}
$$

with $n_{\mathrm{CME}, 0}^{\mathrm{s}}=n_{\mathrm{CME}}^{\mathrm{s}}\left(d=d_{0}\right)=7.1 \cdot 10^{6} \mathrm{~m}^{-3}$, and $d_{0}=1 \mathrm{AU}$.

As far as the CME velocity is concerned, one has to note that individual CMEs have very different velocities. However, the average CME velocity $v$ is approximately independent of the subsolar distance, and is similar for both types of CMEs:

$$
v_{\mathrm{CME}}^{\mathrm{w}}=v_{\mathrm{CME}}^{\mathrm{s}}=v_{\mathrm{CME}}=5.26 \cdot 10^{5} \mathrm{~m} / \mathrm{s} .
$$

Similarly to section 5.1.3, the CME velocity given by eq. (5.20) has to be corrected for the orbital motion of the planet:

$$
v_{\mathrm{eff}, \mathrm{CME}}=\sqrt{\frac{M_{\star} G}{d}+v^{2}}
$$

In addition to the density and the velocity, the temperature of the plasma in a coronal mass ejection will be required in later sections. According to Khodachenko et al. (2006), the front region of a CME consists of hot, coronal material $(T \approx 2 \mathrm{MK})$. This region may either be followed by relatively cool prominence material $(T \approx 8000 \mathrm{~K})$, or by hot flare material $(T \approx 10 \mathrm{MK})$. In the following, the temperature of the leading region of the CME will be used, i.e. $T_{\mathrm{CME}}=2 \mathrm{MK}$.

The values obtained using eq. (5.19) and (5.20) for the plasma density and velocity during a CME and the values for the case of a steady state stellar wind are compared in Section 5.2.3. 


\subsubsection{Occurrence rate}

In the solar system, impacts of CMEs on a planet occur only very rarely. For close-in extrasolar planets, however, Khodachenko et al. (2006) find that CME-planet collisions can be expected to happen much more frequently. In some cases, planets may even be under the continuous influence of CMEs.

The argumentation is as follows (Khodachenko et al. 2006): A CME of angular size $\Delta_{\mathrm{CME}}$ is assumed to propagate strictly radially from its star. The range of latitudes where CMEs occur is restriced to $\pm \Theta$ around the equatorial plane of the star. Then, the frequency $f_{\text {impact }}$ at which CMEs impact on a planet of an angular size $\delta_{\mathrm{p}}$ can be estimated to be (Khodachenko et al. 2006):

$$
f_{\text {impact }}=\frac{\left(\Delta_{\mathrm{CME}}+\delta_{\mathrm{p}}\right) \sin \left(\frac{\Delta_{\mathrm{CME}}+\delta_{\mathrm{p}}}{2}\right)}{2 \pi \sin \Theta} f_{\mathrm{CME}},
$$

where $f_{\mathrm{CME}}$ is the frequency with which CMEs are ejected by the star, and the coefficient in front of $f_{\mathrm{CME}}$ describes the probability for an ejected CME to collide with the planet. A planet can be assumed to be under the permanent influence of CMEs when the time between two successive CMEs is shorter than the duration of a CME, $\tau_{\mathrm{CME}}$. In other words, the CME impact rate has to exceed a critical level given by

$$
f_{\text {impact }} \geq \frac{1}{\tau_{\mathrm{CME}}}
$$

From eqs. 5.22) and 5.23, one can estimate the critical CME production rate $f_{\mathrm{CME}}^{\mathrm{c}}$. If the stellar CME production rate exceeds $f_{\mathrm{CME}}^{\mathrm{c}}$, then the discrete character of CMEplanet encounters is replaced by a continuous influence:

$$
f_{\mathrm{CME}}^{\mathrm{c}}=\frac{2 \pi \sin \Theta}{\left(\Delta_{\mathrm{CME}}+\delta_{\mathrm{p}}\right) \sin \left(\frac{\Delta_{\mathrm{CME}}+\delta_{\mathrm{p}}}{2}\right) \tau_{\mathrm{CME}}} .
$$

To obtain an estimation for the value of $f_{\mathrm{CME}}^{\mathrm{c}}$, extrasolar CMEs are assumed to be similar to solar CMEs, i.e. $\frac{\pi}{3} \leq \Delta_{\mathrm{CME}} \leq \frac{2 \pi}{3}$, and $\tau_{\mathrm{CME}} \approx 8$ hours (obtained from brightness measurements at heliocentric distances of 6-10 solar radii, see Lara et al. 2004). Furthermore, the angular size of the planet is neglected $\left(\delta_{\mathrm{p}} \rightarrow 0\right)$, and CMEs are assumed to be isotropically distributed on the stellar surface ${ }^{4}\left(\Theta=\frac{\pi}{2}\right)$. Thus, for a CME production rate exceeding $f_{\mathrm{CME}}^{\mathrm{c}}$ with

$$
10 \text { day }^{-1} \leq f_{\mathrm{CME}}^{\mathrm{c}} \leq 36 \text { day }^{-1}
$$

one obtains continuous action of CMEs. Note that these values for the critical CME production rate are not much higher than the CME production rate of the present-day Sun at its activity maximum $\left(f_{\mathrm{CME}} \sim 6 \ldots 8 \mathrm{day}^{-1}\right)$. For this reason, the assumption of a permanent influence of stellar CMEs on a close-in planet appears to be realistic.

The numbers given so far strongly differ from the number of CMEs recorded at the Earth's orbit at 1 AU. The reason for this apparent discrepancy is the fact that only a small fraction of the CMEs reach such large orbital distances. Many solar CMEs could not be

\footnotetext{
${ }^{4}$ For the Sun, most CMEs originate in the region around the equator given by $\Theta=\pi / 3$ (Khodachenko et al. 2006). However, the larger value of $\Theta$ is chosen to obtain an upper limit for $f_{\mathrm{CME}}^{\mathrm{c}}$.
} 
tracked by the LASCO instrument on board of SoHO beyond orbital distances of $\approx 0.05$ AU (Khodachenko et al. 2006). Only about 20\% (possibly even less) of all CMEs are strong enough to reach orbital distances of $1 \mathrm{AU}$ and more. Thus, for planets at larger orbital distances, the critical CME production rate given by eq. 5.24) has to be corrected by a distance-dependent factor. However, for close-in extrasolar planets (i.e. for $d \leq 0.1$ $\mathrm{AU})$, all CMEs have to be taken into account, thus strongly increasing the number of CME collisions when compared to the planets of the solar system. Also, note that stars which are different from the Sun (especially younger stars) may exhibit a stronger CME activity than the Sun does.

For close-in planets around stars where the CME occurrence rate exceeds the critical value (i.e. $f_{\mathrm{CME}}>f_{\mathrm{CME}}^{\mathrm{c}}$ ), the planet can be considered to be under continuous influence of CMEs. In this case the speed and density of the stellar wind around the planet have to be replaced by the CME speed and density. This is based on the assumption that the duration of each CME is long enough, so that its action on a planet can be regarded as the action of a stellar wind which has the density and velocity of the CME. The typical duration of a CME is several hours, and the typical reaction time of the magnetosphere is of the order of several minutes. For this reason, this assumption appears to be reasonable, and $n_{\mathrm{CME}}$ and $v_{\mathrm{CME}}$ obtained in Section 5.2.1 can be used analogous to the stellar wind parameters $n$ and $v$.

\subsubsection{Comparison to stellar wind parameters}

In this section, the parameters $n_{\mathrm{CME}}^{\mathrm{s}}$ and $v_{\mathrm{CME}}$ obtained in Section 5.2.1 for strong coronal mass ejections are compared to the corresponding stellar wind values $n$ and $v$, which were derived in Section 5.1.4.

In Fig. 5.3, the distance-dependent parameters $v_{\mathrm{CME}}$ and $n_{\mathrm{CME}}^{\mathrm{s}}$ for strong CMEs are compared to the typical solar wind parameters obtained by the Parker model (already presented in Figure 5.1 of Section 5.1.1.1). One can see that close-in planets are subject to considerably faster and denser plasma environments during a strong coronal mass ejection than during quiet times. The effective plasma velocity is increased by a factor of approximately two to three, while the density differs by almost one order of magnitude for close-in planets.

Similarly to Table 5.1, Table 5.3 compares the velocities and densities for different substellar distances. Again, it becomes obvious that strong CMEs present a parameter regime totally different from the steady stellar wind. For stars with frequent CMEs, this dense and fast environment completely replaces the stellar wind (see Section 5.2.2). The resulting compression of the planetary magnetosphere is discussed in Section 5.3 .2 .

\subsection{Planetary magnetospheres}

The magnetosphere of a planet results from the interaction of the stellar wind with the planetary magnetic field. This section describes the magnetospheric model used within this work. The shape of the model magnetosphere as well as its magnetic field are described in Section 5.3.1. The size of the magnetosphere is determined by the pressure equilibrium at the magnetopause (the boundary layer separating the magnetosphere from 


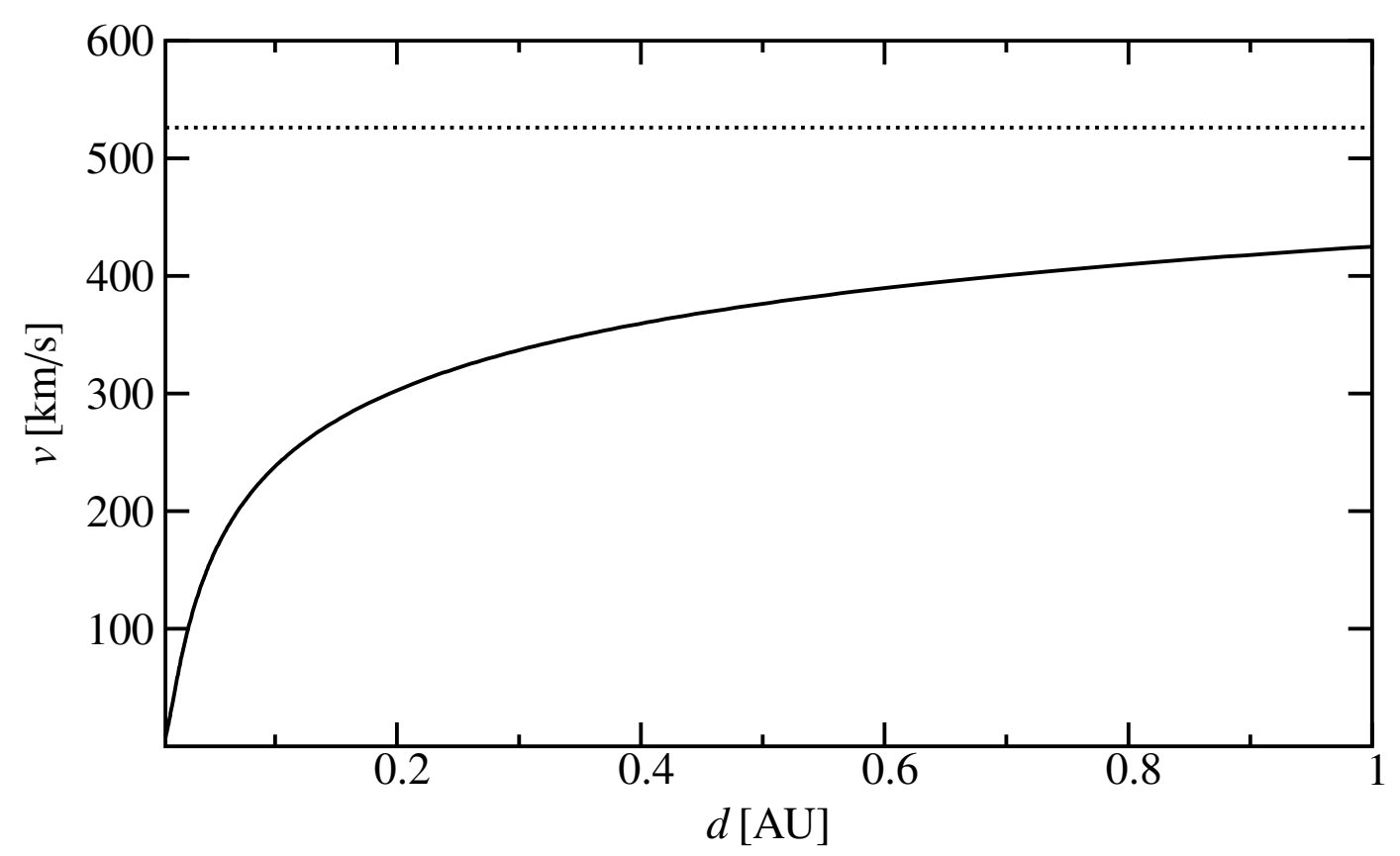

(a) Velocity of a CME (dotted line) compared to the solar wind velocity (solid line).

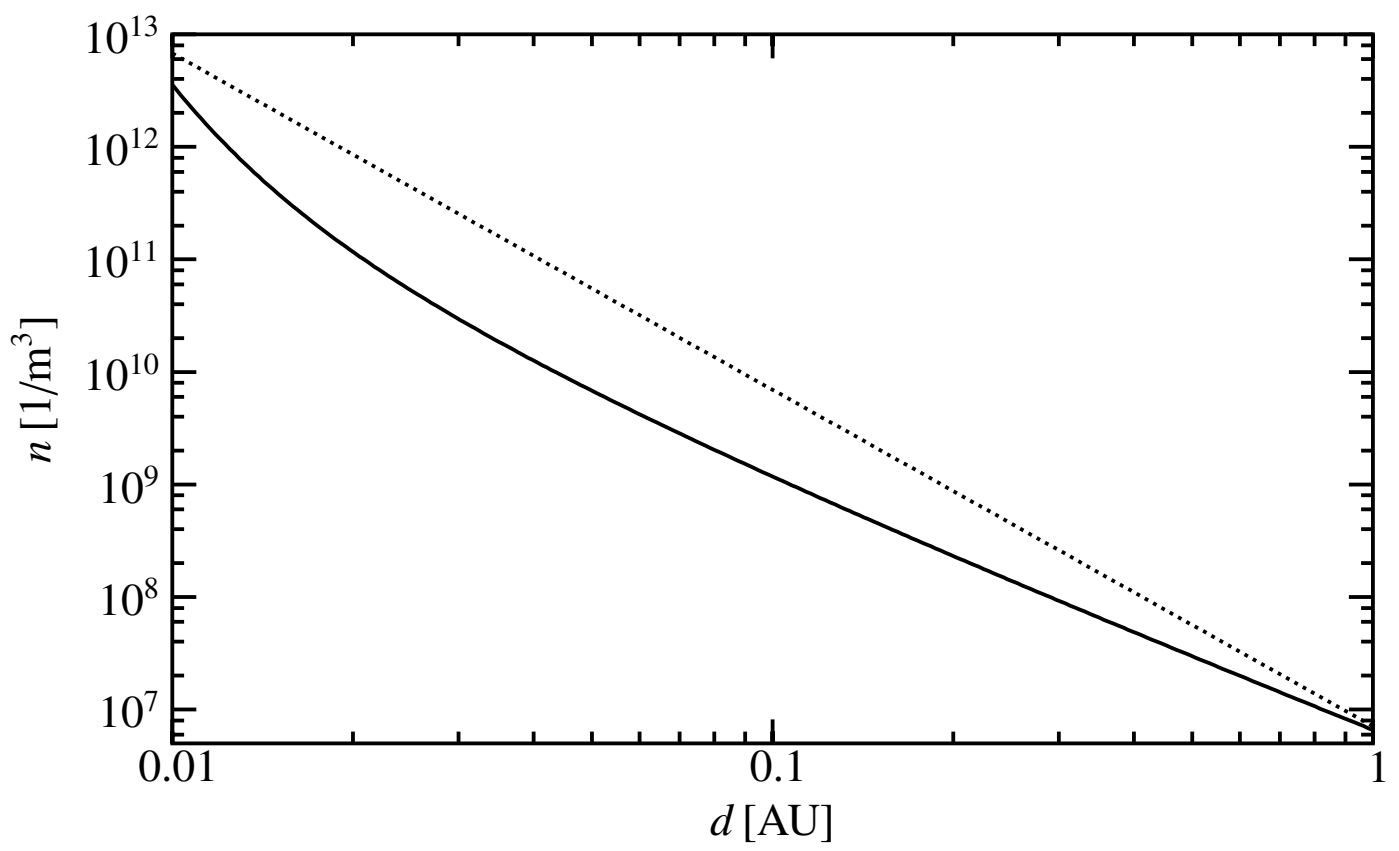

(b) Density of a strong CME (dotted line) compared to the solar wind density (solid line).

Figure 5.3: Velocity $v_{\mathrm{CME}}$ and density $n_{\mathrm{CME}}^{\mathrm{s}}$ of a strong coronal mass ejection (dotted lines) as compared to the solar wind velocity $v$ and density $n$ (solid lines, taken from Section 5.1.1.1).

the stellar wind plasma) in Section 5.3.2. In Section 5.3.3 and 5.3.4, the sizes of sample magnetospheres are calculated for gas giants and for terrestrial exoplanets, respectively. 
5 Formation of magnetospheres by stellar winds

\begin{tabular}{|l||c|c|}
\hline planet around a $\mathrm{G}$ star & stellar wind & stellar CME \\
\hline$v(t)$ (velocity at $0.05 \mathrm{AU})[\mathrm{km} / \mathrm{s}]$ & 164 & 526 \\
$v_{\text {eff }}(t)$ (effective velocity at $\left.0.05 \mathrm{AU}\right)[\mathrm{km} / \mathrm{s}]$ & 212 & 543 \\
$n(t)$ (density at $0.05 \mathrm{AU})\left[\mathrm{m}^{-3}\right]$ & $6.82 \cdot 10^{9}$ & $5.5 \cdot 10^{10}$ \\
\hline$v(t)$ (velocity at $0.2 \mathrm{AU})[\mathrm{km} / \mathrm{s}]$ & 303 & 526 \\
$v_{\text {eff }}(t)$ (effective velocity at $\left.0.2 \mathrm{AU}\right)[\mathrm{km} / \mathrm{s}]$ & 310 & 530 \\
$n(t)$ (density at $0.2 \mathrm{AU})\left[\mathrm{m}^{-3}\right]$ & $2.31 \cdot 10^{8}$ & $8.7 \cdot 10^{8}$ \\
\hline$v(t)($ velocity at $1.0 \mathrm{AU})[\mathrm{km} / \mathrm{s}]$ & 425 & 526 \\
$v_{\text {eff }}(t)$ (effective velocity at $\left.1.0 \mathrm{AU}\right)[\mathrm{km} / \mathrm{s}]$ & 426 & 527 \\
$n(t)$ (density at $1.0 \mathrm{AU})\left[\mathrm{m}^{-3}\right]$ & $6.59 \cdot 10^{6}$ & $7.1 \cdot 10^{6}$ \\
\hline$T($ plasma temperature) $[\mathrm{MK}]$ & 0.81 & 2.0 \\
\hline
\end{tabular}

Table 5.3: Parameters typical for a strong stellar CME at distances of $0.05 \mathrm{AU}, 0.2 \mathrm{AU}$ and 1.0 AU around a Sun-like G type star $\left(M_{\star}=1.0 M_{\odot}\right.$, radius $R_{\star}=1.0 R_{\odot}$, and age $\left.t_{\star}=4.6 \mathrm{Gyr}\right)$, compared to quiet stellar wind parameters.

\subsubsection{Magnetospheric model}

Since the magnetospheric topology of exoplanets is unknown, one has to use magnetospheric models to estimate the size and shape of the magnetosphere of such a planet. The magnetospheric model employed in this work relies on the potential field model which was developed by Voigt (1981) and extended by Stadelmann (2005a). Its first application to extrasolar planets was described by Grießmeier et al. (2005b). This section gives a brief summary of the most important properties of this model.

In the magnetospheric model of Stadelmann (2005a), the shape of the magnetosphere is not self-consistent, but given by a semi-infinite cylinder on the nightside and a hemisphere on the dayside. Such a magnetosphere is schematically shown in Fig. 5.4. Both the cylinder and the hemisphere have the same radius, $R_{\mathrm{M}}$, which will be determined in Section 5.3.2. The planet is not located exactly at the boundary between the cylinder and the hemisphere, but is shifted into the hemisphere. Thus, its distance to the magnetopause (the standoff distance $R_{\mathrm{s}}$ ) is smaller than the radius $R_{\mathrm{M}}$ of the hemisphere: $R_{\mathrm{s}}<R_{\mathrm{M}}$. According to observations and models, $R_{\mathrm{s}} \approx 0.5 R_{\mathrm{M}}$ for Jupiter (e.g. Joy et al. 2002). As a first-order approximation, self-similarity is satisfied (Vogt and Glassmeier 2001, Glassmeier et al. 2004), so that this ratio will be assumed for all planets:

$$
R_{\mathrm{s}}=\frac{R_{\mathrm{M}}}{2}
$$

The coordinate axes are chosen as follows: the orbital plane of the planet is the $y$ - $z$-plane, where the star and the planet are aligned on the $z$-axis. The origin of the coordinate system is centred on the plane connecting the cylinder and the hemisphere. This corresponds to the M-coordinate system of Stadelmann (2005a, Figure 3.1). 


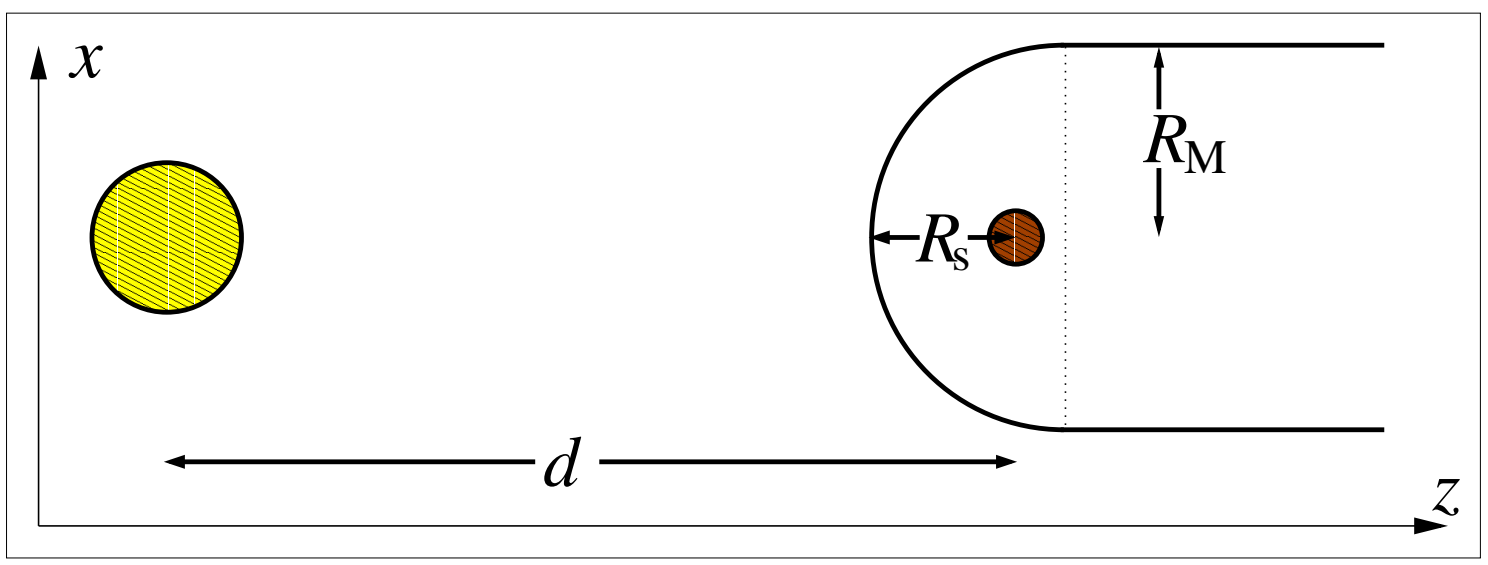

Figure 5.4: Geometry of the magnetospheric model (schematic view).

The magnetospheric magnetic field of the planet is the result of a superposition of an internal and an external part. The internal part of the magnetic field is generated by magnetohydromagnetic processes in the planetary core. As described in Sections 3.3 and 4. it will be assumed that the internal contribution to the magnetic field can be described by a zonal dipole moment centred within the planet. This dipole is oriented parallel to the $x$-axis. In the case of the Earth, the external magnetic field is caused by current systems, mainly consisting of the equatorial ring current, the tail currents and the magnetopause currents, i.e. the Chapman-Ferraro currents (Jordan 1994). Many models with different advantages and disadvantages have been developed to describe the resulting planetary magnetic field configuration. A model using a potential field ansatz for the description of the external magnetic field was developed by Voigt (1981) and later extended by Stadelmann (2005a). The advantage of this model is that it can easily be adopted to study the magnetic field geometry of planets differing in magnetic moment and magnetic topology, which is necessary for the purpose of this work.

Within this magnetic field model, most of the external magnetic field contributions can be considered: In the far field the magnetic field of the ring current can be modelled like a dipole field (Stern 1985). Thus, no additional term for this field contribution is required. The magnetic field $\underline{B}_{\mathrm{j}}$ generated by the tail currents can be modelled by stretching the magnetic field lines into the deep tail. Since the tail currents have no effect on the dayside part of the magnetosphere and have only little influence on the trajectories of high energetic particles (Stadelmann 2005a, Figure 5.12), the contribution of $\underline{B}_{\mathrm{j}}$ is neglected in this work. Additional field contributions are generated by currents on the magnetopause. For this work, the magnetospheric current system is reduced to the Chapman-Ferraro currents. The magnetic field $\underline{B}_{\text {cf }}$ caused by the Chapman-Ferraro currents shields the planetary magnetic field against the interplanetary magnetic field $\underline{B}_{\text {imf }}$. Thus, the total magnetic field is constructed by superposing the internal field $\underline{B}_{\mathrm{p}}$ and the field of the magnetopause currents $\underline{B}_{\mathrm{cf}}$ :

$$
\underline{B}=\underline{B}_{\mathrm{p}}+\underline{B}_{\mathrm{cf}} .
$$

In the following, a closed magnetosphere is assumed, so that field lines are not allowed to pass through the magnetopause. In mathematical terms, this means that the normal 
component of the total magnetic field $\underline{B}$ has to vanish on the magnetopause.

The model magnetosphere is taken to be static, so that all time-derivatives vanish. With $\nabla \times \underline{B}=0$ inside the magnetosphere, the magnetic field $\underline{B}$ can be derived from a scalar potential $u$ :

$$
\underline{B}=-\mu_{0} \nabla u \text {. }
$$

Together with Maxwell's equation $\nabla \cdot \underline{B}=0$, this results in the Laplace equation for the scalar potential

$$
\Delta u=0 .
$$

To determine the potential $u$ and then, using eq. (5.28), the magnetic field $\underline{B}$ within the magnetopause, equation (5.29) has to be solved under the following boundary conditions:

1. As described above, the normal component of the total magnetic field has to vanish on the magnetopause:

$$
\underline{\hat{n}} \cdot \underline{B}=\underline{\hat{n}} \cdot \underline{B}_{\mathrm{p}}+\underline{\hat{n}} \cdot \underline{B}_{\mathrm{cf}} \equiv 0 .
$$

2. Furthermore, the magnetic field has to vanish at infinity, i.e. at the open end of the cylinder.

$$
B(z \rightarrow \infty)=0
$$

3. Finally, continuity of the potential is required at the connecting plane between the hemisphere and the cylinder.

$$
u_{\text {hemisphere }}(x, y, z=0)=u_{\text {cylinder }}(x, y, z=0) .
$$

The solution of the Laplace equation (5.29) under the given boundary conditions (5.30), 5.31) and 5.32) is obtained as follows: The internal magnetic field is described by a zonal magnetic dipole, which is taken from Section 4.4 or Section 4.6 (depending on the type of the planet). The normal component of this internal magnetic field $\underline{B}_{\mathrm{p}}$ is calculated for the magnetopause in the hemisphere. In order to satisfy the first boundary condition eq. (5.30), the additional magnetic field $\underline{B}_{\mathrm{cf}}$ is constructed such that the normal component of the total magnetic field $\underline{B}=\underline{B}_{\mathrm{p}}+\underline{B}_{\mathrm{cf}}$ vanishes on the magnetopause. Since currents outside the magnetopause are not considered, the field $\underline{B}_{\mathrm{cf}}$ generated by the Chapman-Ferraro currents can be derived from a scalar potential using spherical harmonics. For the total magnetic field $\underline{B}$ in the cylinder, a potential field ansatz using Bessel functions is made such that the boundary conditions eq. (5.30) and eq. (5.31) are fulfilled. The coefficients of the Bessel functions are determined by satisfying the last boundary condition, eq. (5.32). The interested reader is referred to Stadelmann (2005a) for more details. An example for the resulting magnetic field configuration is shown in Figure 5.5.

\subsubsection{Pressure equilibrium}

With the shape of the magnetopause described in Section 5.3.1, only its size remains to be determined. For this purpose, the size of the magnetosphere is assumed to be constant on large timescales (i.e. Myr). In reality, short-term fluctuations of the stellar wind 


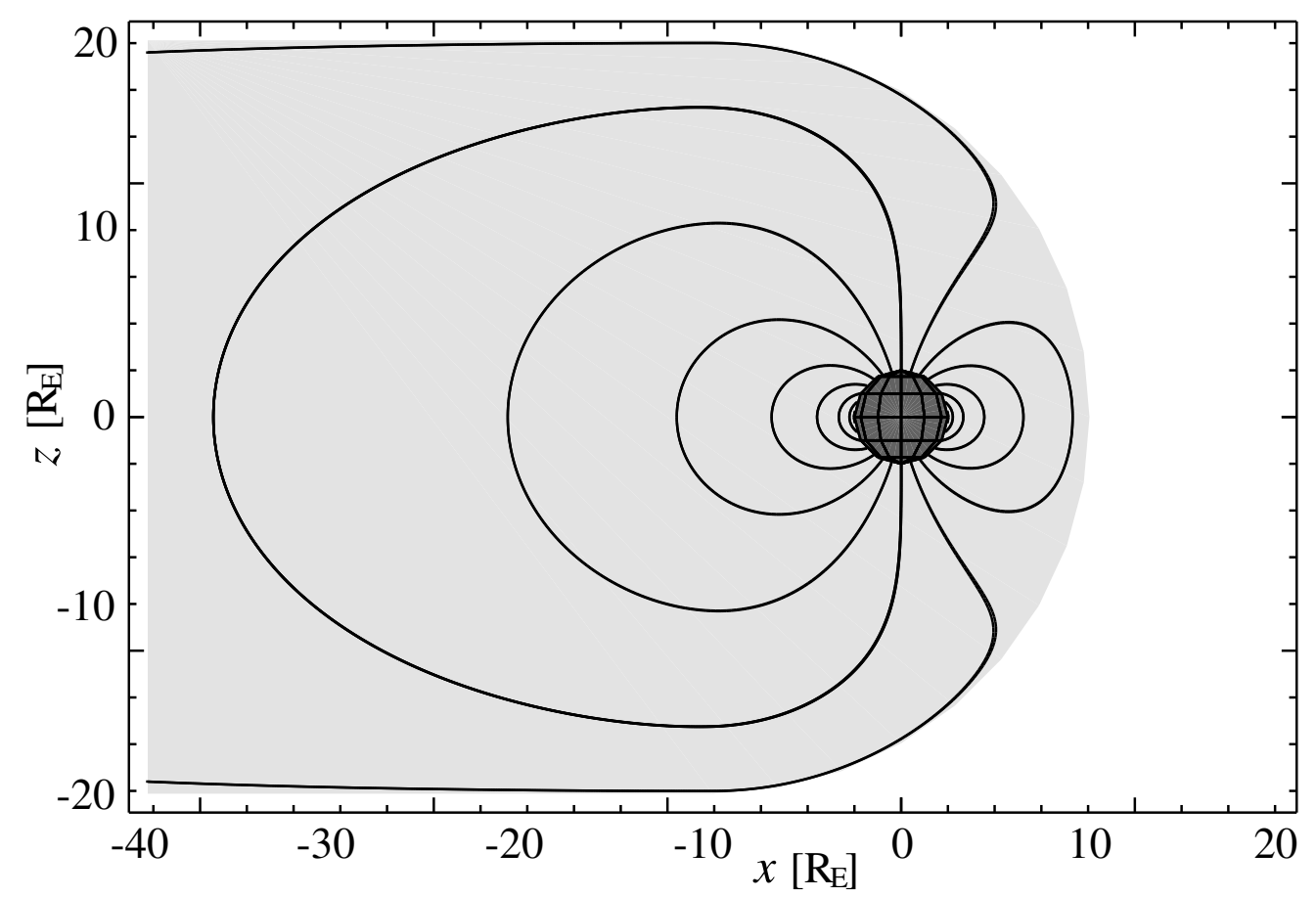

Figure 5.5: Magnetospheric magnetic field for a tidally locked extrasolar planet.

parameters (velocity and density) will lead to rapid changes in the size of the magnetosphere. Here, however, average stellar wind conditions are used, so that the average size of a magnetosphere is calculated.

The magnetosphere of a planet results from the interaction of the stellar wind with the planetary magnetic field. The size of the magnetopause is determined by the pressure equilibrium between the pressure exerted by the stellar wind and the magnetic field pressure on the magnetopause (to which both the planetary magnetic field and the field generated by Chapman-Ferraro currents contribute). More precisely, the standoff distance $R_{\mathrm{s}}$, i.e. the planetocentric distance of the magnetopause along the line connecting the planet and the star is defined as the point along the direction of $\underline{v}_{\text {eff }}$, where a pressure equilibrium is satisfied. The following contributions to the pressure balance have to be carefully analysed:

- the stellar wind ram pressure $p_{\mathrm{sw}}$ (i.e. the pressure introduced by the bulk velocity of the stellar wind particles).

- the magnetic field pressure $p_{\text {imf }}$ caused by the interplanetary magnetic field originating from the star.

- the thermal plasma pressure in the stellar wind $p_{\text {th }}$.

- the magnetic field pressure $p_{\mathrm{m}}$ caused by the planetary magnetic field, including both the planetary dipole field and the magnetic field generated by the ChapmanFerraro currents.

- the thermal pressure of the magnetospheric plasma $p_{\mathrm{th}, \mathrm{m}}$. 
Thus, the full pressure balance is given by

$$
p_{\mathrm{sw}}+p_{\mathrm{imf}}+p_{\mathrm{th}}=p_{\mathrm{m}}+p_{\mathrm{th}, \mathrm{m}} .
$$

In the following, each contribution to eq. 5.33 will be briefly discussed in a separate section. Then, the overall pressure balance is given in Section 5.3.2.6.

\subsubsection{Stellar wind kinetic pressure}

The stellar wind ram pressure (also called "dynamic pressure" or "kinetic pressure") can be expressed as

$$
p_{\text {sw }}=m n v^{2},
$$

where $m$ denotes the mass of the stellar wind protons, $n$ is their number density, and $v$ is their velocity. To be exact, at the magnetopause the velocity normal to the magnetopause is zero, and the pressure equilibrium is maintained by magnetic and thermal contributions. However, this pressure is approximately identical to the stellar wind ram pressure further upstream (Walker and Russell 1995, Prölss 2001).

The stellar wind quantities $n$ and $v$ were already discussed in Section 5.1. For stellar coronal mass ejections, $n_{\mathrm{CME}}$ and $v_{\mathrm{CME}}$ can be used analogously, see Section 5.2. To correctly take into account the relative velocity between the planetary magnetosphere and the stellar wind plasma, $v$ has to be replaced by $v_{\text {eff }}$ in eq. (5.34). Thus, $p_{\mathrm{sw}}$ is given by

$$
p_{\mathrm{sw}}=m n v_{\mathrm{eff}}^{2} \cdot
$$

In the solar system, the location of the magnetopause is controlled by the dynamic pressure of the solar wind. For the Earth, the contributions of $p_{\text {imf }}$ and $p_{\text {th }}$ to the total external pressure are negligible (Walker and Russell 1995, Baumjohann and Treumann 1999). For close-in planets, however, these additional pressure contributions are relevant and have to be evaluated. This will be done in the following sections.

\subsubsection{Stellar wind magnetic pressure}

At $1 \mathrm{AU}$, the average field strength of the interplanetary magnetic field is $B_{\mathrm{imf}} \approx 3.5 \mathrm{nT}$ (Mariani and Neubauer 1990, Prölss 2001). The corresponding magnetic pressure is two orders of magnitude smaller than the kinetic pressure of the stellar protons. Thus, for Earth, the contribution of the interplanetary magnetic field is not the dominating effect, and $p_{\text {imf }}$ can be neglected in the pressure equilibrium (5.33).

The same is not necessarily true for smaller substellar distances $d$. According to the Parker stellar wind model (Parker 1958), the radial component of the interplanetary magnetic field decreases as

$$
B_{\mathrm{imf}, r}(d)=B_{r, 0}\left(\frac{d}{d_{0}}\right)^{-2} .
$$

This was later confirmed by Helios measurements. One finds $B_{r, 0} \approx 2.6 \mathrm{nT}$ and $d_{0}=1$ AU (Mariani and Neubauer 1990, Prölss 2001). At the same time, the azimuthal component $B_{\mathrm{imf}, \varphi}$ behaves as

$$
B_{\mathrm{imf}, \varphi}(d)=B_{\varphi, 0}\left(\frac{d}{d_{0}}\right)^{-1}
$$


with $B_{\varphi, 0} \approx 2.4 \mathrm{nT}$ (Mariani and Neubauer 1990, Prölss 2001). Also, the average value of $B_{\mathrm{imf}, \theta}$ vanishes ( $B_{\mathrm{imf}, \theta} \approx 0$ ), so that for small distances $d$ the quantity $B_{\mathrm{imf}}^{2} / 2 \mu_{0}$, which is usually associated with the magnetic pressure, varies as $B_{\text {imf }}^{2} / 2 \mu_{0} \propto d^{-4}$. Comparing this to the stellar wind ram pressure, which roughly scales as $p_{\text {sw }} \propto d^{-2}$, the stellar magnetic pressure appears to be dominating for locations closer than a certain critical distance. This critical distance is located at approximately 0.1 AU (Stracke 2004, Figure 3.9).

However, one has to take into account the orientation of the interplanetary magnetic field. Obviously, with $B_{\mathrm{imf}, r}(d)$ and $B_{\mathrm{imf}, \varphi}(d)$ given by eqs. (5.36) and (5.37), the angle between the stellar wind flow $\underline{v}$ and the interplanetary magnetic field $\underline{B}_{\text {imf, }}$ is a function of substellar distance. At small orbital distances, $B_{\mathrm{imf}, \varphi} \ll B_{\mathrm{imf}, r}$ so that $\underline{v}$ and $\underline{B}_{\text {imf }}$ are approximately parallel. The angle $\chi$ between $\underline{B}_{\mathrm{imf}}$ and the radial direction is given by

$$
\chi(d)=\arctan \left[\frac{B_{\mathrm{imf}, \varphi}(d)}{B_{\mathrm{imf}, r}(d)}\right] .
$$

Thus, for $d=0.2 \mathrm{AU}, \chi \approx 10^{\circ}$, and for $d=0.05 \mathrm{AU}, \chi \approx 3^{\circ}$. In other words, $\underline{v}$ and $\underline{B}_{\text {imf }}$ are approximately parallel for close-in planets.

Petrinec and Russell (1997) describe the position of the magnetopause for specific orientations of the upstream interplanetary magnetic field. For $\underline{B}_{\text {imf }} \| \underline{v}$, they find that the substellar standoff distance is not influenced by the magnetic field. For the general case, where the interplanetary magnetic field and the stellar wind flow are neither parallel nor perpendicular, no analytical solution is presently known. Because for close-in exoplanets $\underline{v}$ and $\underline{B}_{\mathrm{imf}}$ are approximately parallel, one finds

$$
p_{\text {imf }} \approx 0
$$

The strong radial component of the interplanetary magnetic field poses a problem for the magnetospheric model presented in Section 5.3.1. In fact, with $B_{\mathrm{imf}, r} \gg B_{\mathrm{imf}, \varphi}$, the approximations on which this model relies are no longer satisfied (Voigt 1979, 1995) and the closed magnetosphere will be opened by magnetic reconnection (i.e. the merging of magnetic field lines) at the substellar point. There is no analytical model which describes such a situation satisfactorily, so that the only way seems to be to resort to MHD simulations. First MHD simulations of close-in exomagnetospheres were recently performed, and indeed exhibit a modified magnetospheric topology because of magnetic reconnection (Ip et al. 2004, Zuchowski 2005, Preusse 2006). The current work aims at providing simple analytical estimations. Preusse (2006, Section 5.3.2) shows that the location of the magnetopause found by MHD simulations deviates from that derived using a pressure balance (as done in the present work). This is especially true for orbital distances below $0.04 \mathrm{AU}$, where the error in $R_{\mathrm{s}}$ may exceed one planetary radius. Keeping this limitation in mind, the results given in this work should be regarded as a first order approximation.

\subsubsection{Stellar wind thermal pressure}

Another contribution to the total external pressure is given by the stellar wind thermal pressure. The pressure of the stellar wind ions can be written as:

$$
p_{\text {th,ion }}=n k_{\mathrm{B}} T \text {, }
$$


where $k_{\mathrm{B}}$ is Boltzmann's constant, and $n$ and $T$ are the stellar wind density and temperature at the location of the planet, respectively. Because of the quasineutrality of the stellar wind plasma, and assuming that protons and electrons have the same temperature, the total thermal pressure is given by:

$$
p_{\text {th }}=2 n k_{\mathrm{B}} T
$$

In the solar system, this contribution is negligible. For close-in planets, however, the stellar wind velocity is much lower, see Section 5.1. which reduces the relative contribution of the kinetic pressure $p_{\mathrm{sw}}$. Moreover, for large distances the stellar wind temperature is much smaller than the corona temperature (Marsch 1991). Stracke (2004, Figure 3.9) shows that $p_{\text {th }}$ exceeds the contribution of the dynamic pressure $p_{\mathrm{sw}}$ for orbital distances $\lesssim 0.04$ AU.

For the calculation of $p_{\text {th }}$, the stellar wind density $n(d)$ is taken from Section 5.1. In the isothermal Parker stellar wind model used here, the plasma temperature $T$ is constant and identical to the stellar corona temperature, which is also obtained in Section 5.1. For a planet under the influence of CMEs, both $n_{\mathrm{CME}}$ and $T_{\mathrm{CME}}$ are taken from Section 5.2 .

\subsubsection{Planetary magnetic pressure}

The magnetic pressure of the magnetic field parallel to the surface of the magnetopause is given by

$$
p_{\mathrm{m}}=\frac{\underline{B}^{2}}{2 \mu_{0}}
$$

where $\underline{B}$ is the total magnetic field at the magnetopause. According to eq. 5.27) in Section 5.3.1, $\underline{B}$ is obtained as a superposition of the planetary magnetic field with an external magnetic field driven by the magnetopause currents: $\underline{B}=\underline{B}_{\mathrm{p}}+\underline{B}_{\mathrm{cf}}$.

As described in Sections 3.3 and 4, it is assumed that the internal contribution to the magnetic field $\underline{B}_{\mathrm{p}}$ can be described by a zonal magnetic dipole moment $\mathcal{M}$ centred within the planet. The value of $\mathcal{M}$ will be taken from Section 4.4 or Section 4.6 (depending on the type of planet). Then, the absolute value of $B_{\mathrm{p}}$ at the substellar point of the magnetopause is given by:

$$
B_{\mathrm{p}}=\frac{\mu_{0}}{4 \pi} \frac{\mathcal{M}}{R_{\mathrm{s}}^{3}}
$$

Here, $R_{\mathrm{s}}$ is the standoff distance (i.e. the distance of the magnetopause to the planetary centre along the direction of $\underline{v}_{\text {eff }}$ ).

As described in Section 5.3.1, the magnetopause currents are present only on the boundary, so that their magnetic field can be deduced from a scalar potential. This potential meets the boundary condition that the normal component of the magnetospheric magnetic field vanishes at the magnetopause. For a spherical magnetopause this potential can be described by spherical harmonics. At the substellar point the expression for the magnetic field is similar to the planetary magnetic field of eq. (5.43), so that the total magnetic field can be written as

$$
\underline{B}_{\mathrm{p}}+\underline{B}_{\mathrm{cf}}=: 2 f_{0} \underline{B}_{\mathrm{p}}
$$


The form factor $f_{0}$ introduced in eq. (5.44) is $f_{0}=1.5$ for a spherical magnetosphere (Voigt 1995). For a more realistic magnetopause shape, a factor $f_{0}=1.16$ is given by Voigt (1995). This value will be used in the following. Inserting eqs. (5.43), (5.27) and 5.44 into eq. (5.42), one finds for the magnetic pressure of the magnetopause:

$$
p_{\mathrm{m}}=\frac{2 f_{0}^{2} B_{\mathrm{p}}^{2}}{\mu_{0}}=\frac{\mu_{0} f_{0}^{2} \mathcal{M}^{2}}{8 \pi^{2} R_{\mathrm{s}}^{6}} .
$$

\subsubsection{Planetary plasma thermal pressure}

For the magnetised planets of the solar system (with the only exception of Jupiter), the plasma pressure inside the magnetosphere $p_{\mathrm{th}, \mathrm{m}}$ is less than the magnetic pressure $p_{\mathrm{m}}$ of the planetary magnetic field (Walker and Russell 1995). While the same can be expected for extrasolar terrestrial planets, the question remains whether this still holds for close-in giant planets.

For the Hot Jupiter HD 209458b, an expanded upper atmosphere with a size of up to three planetary radii was both observed (Vidal-Madjar et al. 2003, 2004) and predicted from theory (Lammer et al. 2003). Besides the strong stellar XUV flux, which heats the upper atmosphere and leads to hydrodynamic conditions (Lammer et al.2003), the small size of the magnetosphere allows for an increased loss through ion pick-up (Grießmeier et al. 2004, Erkaev et al. 2005). Also, for extremely small orbital distances, the planetary Roche lobe (the zone dominated by the planetary rather than the stellar gravitation) becomes comparable in size to the expanded planetary atmosphere. This leads to geometrical blow-off rather than hydrodynamical blow-off (Lecavelier des Etangs et al. 2004, Jaritz et al. 2005), which is likely to reduce the total mass loss (Jaritz et al.|2005).

With a mass flux of more than $10^{7} \mathrm{~kg} / \mathrm{s}$ from the planet, a contribution of the magnetospheric plasma to the total pressure balance (5.33) cannot be definitely excluded. However, the atmospheric loss is dominated by neutral hydrogen, which does not directly contribute to the plasma pressure. The ionic contribution is at least one order of magnitude below the loss rate of neutral hydrogen (Erkaev et al.|2005). For this reason, $p_{\text {th,m }}$ will be neglected in the pressure balance:

$$
p_{\text {th,m }} \approx 0 \text {. }
$$

\subsubsection{Pressure balance}

With eqs. (5.35), (5.39), (5.41), (5.45) and (5.46), the pressure equilibrium (5.33) can be rewritten as:

$$
m n v_{\mathrm{eff}}^{2}+2 n k_{\mathrm{B}} T=\frac{\mu_{0} f_{0}^{2} \mathcal{M}^{2}}{8 \pi^{2} R_{\mathrm{s}}^{6}} .
$$

For a given planetary orbital distance $d$, only the magnetospheric magnetic pressure is a function of the distance to the planet, while the other factors are constant. Thus, from the pressure equilibrium eq. (5.47) the standoff distance $R_{\mathrm{s}}$ is found to be

$$
R_{\mathrm{s}}=\left[\frac{\mu_{0} f_{0}^{2} \mathcal{M}^{2}}{8 \pi^{2}\left(m n v_{\mathrm{eff}}^{2}+2 n k_{\mathrm{B}} T\right)}\right]^{1 / 6} .
$$


Note that in a few cases, especially for planets with very weak magnetic moments and/or subject to dense and fast stellar winds of young stars, eq. (5.48) yields standoff distances $R_{\mathrm{s}}<R_{\mathrm{p}}$. In those cases, $R_{\mathrm{s}}$ is set equal to $R_{\mathrm{p}}$, because the magnetosphere cannot be compressed to sizes smaller than the planetary radius.

Usually, for solar system problems, only equations (5.35) and (5.45) are inserted into the pressure equilibrium of eq. (5.33), and all other contributions are neglected. In addition, $v$ is taken to be independent of orbital distance, so that $n \propto d^{-2}$. Thus, the well known, simplified scaling for the planetocentric magnetopause distance is obtained:

$$
R_{\mathrm{s}} \propto \mathcal{M}^{1 / 3} d^{1 / 3}
$$

\subsubsection{Size of the magnetosphere of gas giants}

In this section, the sizes of the magnetospheres of Hot Jupiters are estimated for different stellar system ages. This expands and updates the treatment of Grießmeier et al. (2004).

Of the variables appearing in eq. (5.48), $n, v$ and $T$ are taken to be functions of the stellar age (see Section 5.1), while all other parameters are kept constant. The stellar parameters (mass and radius) and the orbital distance of the planet which are required for the calculation of the stellar wind are taken from Table 2.2, whereas the magnetic moment $\mathcal{M}$ is taken from Section 4.4. The standoff distance during a CME encounter is computed with the numbers for $n_{\mathrm{CME}}^{\mathrm{s}}, v_{\mathrm{CME}}$ and $T_{\mathrm{CME}}$ given in Section 5.2

Table 5.4 summarises and compares the standoff distances resulting from eq. (5.47). Instead of using the true age of the planetary system (which is not always known, see Table 2.2), results for three different stellar ages are compared, namely 4.6 Gyr, 1.0 Gyr, and 0.7 Gyr. The corresponding standoff distances are labelled $R_{\mathrm{s}}^{4.6}, R_{\mathrm{s}}^{1.0}$ and $R_{\mathrm{s}}^{0.7}$, respectively. The standoff distance arising from a planet-CME interaction is denoted by $R_{\mathrm{s}}^{\mathrm{CME}}$. Because the CME model of Section 5.2 was constructed for close-in orbital distances, it cannot be applied to Jupiter.

From Table 5.4, the following statements can be obtained:

- The size of the magnetosphere predicted for Jupiter is much smaller than the value obtained from measurements, which, depending on the solar wind conditions, lies in the range $R_{\mathrm{s}}=45 \ldots 100 R_{\mathrm{p}}$ (Lanzerotti and Krimigis 1985, Russell and Walker 1995). This apparent discrepancy is caused by Jupiter's satellite Io, which continuously injects material into the Jovian magnetosphere. Together with the rapid rotation of the planet, this leads to a considerable deviation from the simple pressure balance used here.

- The dense and fast stellar winds of young stars lead to a considerable compression of the planetary magnetospheres.

- For weakly magnetised planets (like HD 209458b, if the lower limit of the magnetic moment estimation is used), the standoff distance can be compressed down to the planetary surface. In this case, a totally different type of interaction will occur.

- For all planets in this list (except for $\tau$ Bootes), young stellar winds compress the magnetosphere down to levels of $\lesssim 3 R_{\mathrm{p}}$, which is the size of the expanded upper atmosphere of HD 209458b, see Section 5.3.2.5. For HD 209458b, this is even the 


\begin{tabular}{|l||c|c|c||c|}
\hline Planet & $R_{\mathrm{s}}^{4.6}\left[R_{\mathrm{p}}\right]$ & $R_{\mathrm{s}}^{1.0}\left[R_{\mathrm{p}}\right]$ & $R_{\mathrm{s}}^{0.7}\left[R_{\mathrm{p}}\right]$ & $R_{\mathrm{s}}^{\mathrm{CME}}\left[R_{\mathrm{p}}\right]$ \\
\hline \hline Jupiter & 40.1 & 22.7 & 19.9 & - \\
HD 209458b & $1.67 \ldots 2.63$ & $1.0 \ldots 1.54$ & $1.0 \ldots 1.35$ & $1.0 \ldots 1.46$ \\
OGLE-TR-10b & $2.31 \ldots 3.48$ & $1.36 \ldots 2.05$ & $1.19 \ldots 1.79$ & $1.20 \ldots 1.81$ \\
OGLE-TR-56b & $3.52 \ldots 4.11$ & $2.39 \ldots 2.79$ & $2.12 \ldots 2.47$ & $1.97 \ldots 2.30$ \\
OGLE-TR-111b & $3.41 \ldots 5.25$ & $1.95 \ldots 3.01$ & $1.71 \ldots 2.63$ & $1.68 \ldots 2.59$ \\
OGLE-TR-113b & $4.76 \ldots 5.78$ & $2.99 \ldots 3.63$ & $2.63 \ldots 3.19$ & $2.19 \ldots 2.65$ \\
OGLE-TR-132b & $3.68 \ldots 4.59$ & $2.38 \ldots 2.97$ & $2.10 \ldots 2.62$ & $2.25 \ldots 2.81$ \\
TrES-1b & $4.01 \ldots 5.66$ & $2.35 \ldots 3.32$ & $2.06 \ldots 2.90$ & $1.93 \ldots 2.73$ \\
$\tau$ Bootes b (light) & $5.02 \ldots 6.79$ & $3.00 \ldots 4.05$ & $2.63 \ldots 3.55$ & $3.11 \ldots 4.20$ \\
$\tau$ Bootes b (medium) & $4.85 \ldots 6.29$ & $2.89 \ldots 3.75$ & $2.53 \ldots 3.29$ & $3.00 \ldots 3.89$ \\
$\tau$ Bootes b (heavy) & $5.42 \ldots 7.06$ & $3.23 \ldots 4.21$ & $2.83 \ldots 3.69$ & $3.35 \ldots 4.37$ \\
\hline
\end{tabular}

Table 5.4: Magnetospheric standoff distances for different Hot Jupiters (Jupiter is shown for comparison), given in planetary radii. Second to fourth column: Standoff distances for Hot Jupiters subject to stellar winds of stars with different ages (4.6 Gyr, $1.0 \mathrm{Gyr}, 0.7 \mathrm{Gyr})$. Fifth column: Standoff distances $R_{\mathrm{S}}^{\mathrm{CME}}$ for Hot Jupiters subject to stellar coronal mass ejections. Note that the CME model of Section 5.2 is not applicable for large orbital distances (e.g. for Jupiter).

case for a stellar age of 7.0 Gyr (see Figure 5.7 below), which corresponds to the upper limit for the estimated stellar age (Mazeh et al. 2000, Cody and Sasselov 2002). This results in an increased atmospheric mass loss through ion pick-up (Grießmeier et al. 2004, Erkaev et al.2005).

- For magnetospheres under the influence of CMEs, the reduction of the standoff distance is comparable to the case of the stellar wind of a young star $\left(t_{\star} \approx 1.0 \mathrm{Gyr}\right)$. Because frequent CME collisions are expected at small orbital distances (see Section 5.2.2, it is possible that $R_{\mathrm{s}}^{\mathrm{CME}}$ effectively replaces $R_{\mathrm{s}}^{4.6}$, the standoff distance for the interaction with a solar-like stellar wind.

The dependence of the standoff distance on the solar system age is also shown in Figure 5.6 for the planet Jupiter. Because of the large orbital radius, the standoff distance is much larger than the planet itself, even for the much stronger stellar wind emanating from the young Sun.

Similarly to Figure 5.6, Figures 5.7 and 5.8 give the standoff distance $R_{\mathrm{s}}$ as a function of the stellar system age $t$ for the two extreme cases of Table 5.4. namely the planets HD $209458 \mathrm{~b}$ and $\tau$ Bootes $\mathrm{b}$ (using the heavy model for the latter, see Section 2.6.2). The area between the minimum and maximum value for $R_{\mathrm{s}}$ is shaded in grey. The dotted line represents the location of the planetary surface $\left(1.0 R_{\mathrm{p}}\right)$. The additional dotted line in Fig. 5.7 represents the size of the observed atmosphere of HD 209458b (3.0 $R_{\mathrm{p}}$, see Section 5.3.2.5). For standoff distances smaller than this limit, enhanced mass loss due to stellar wind ion pick-up can be expected. While the atmosphere of $\tau$ Bootes b seems 


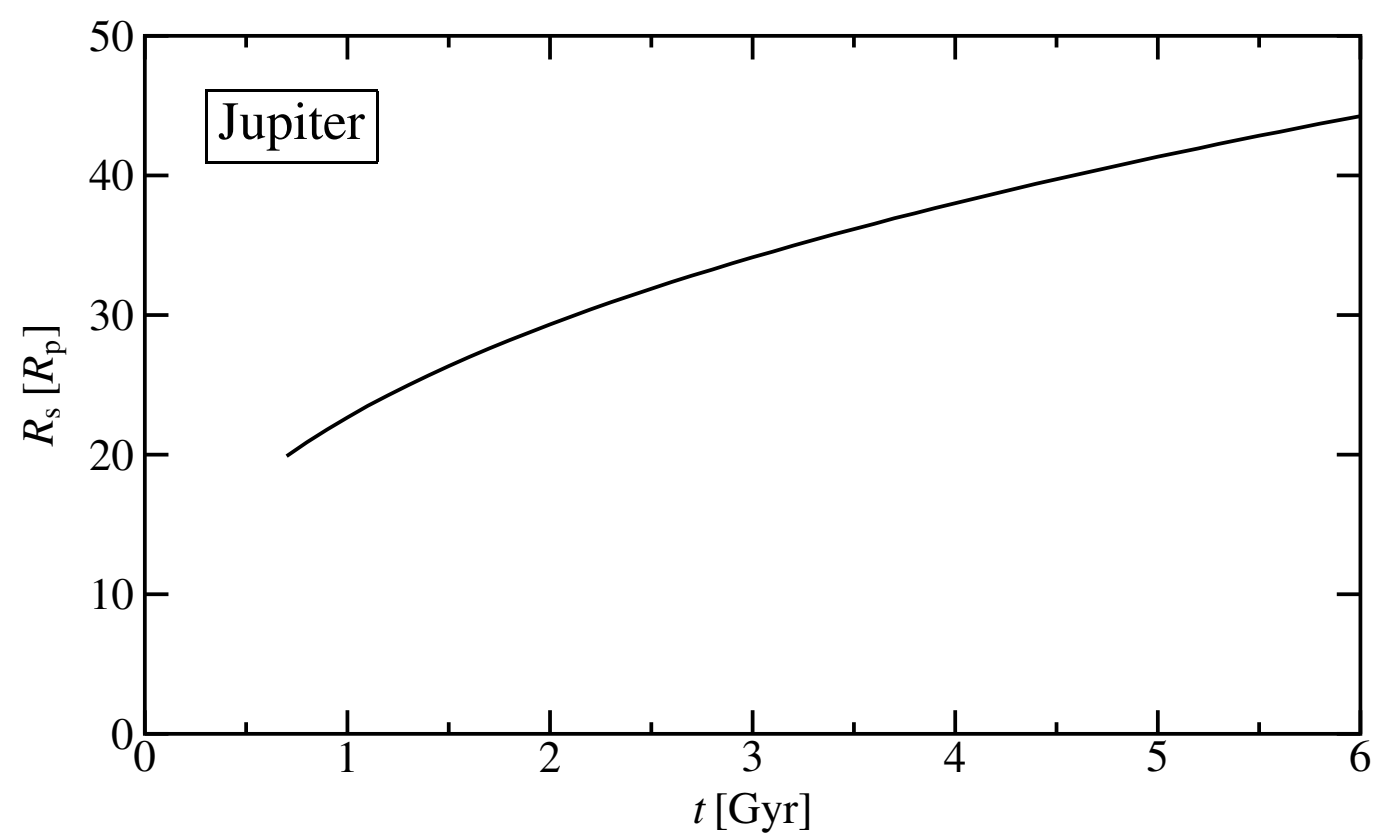

Figure 5.6: Estimated magnetospheric standoff distance for Jupiter as a function of stellar age, given in planetary radii.

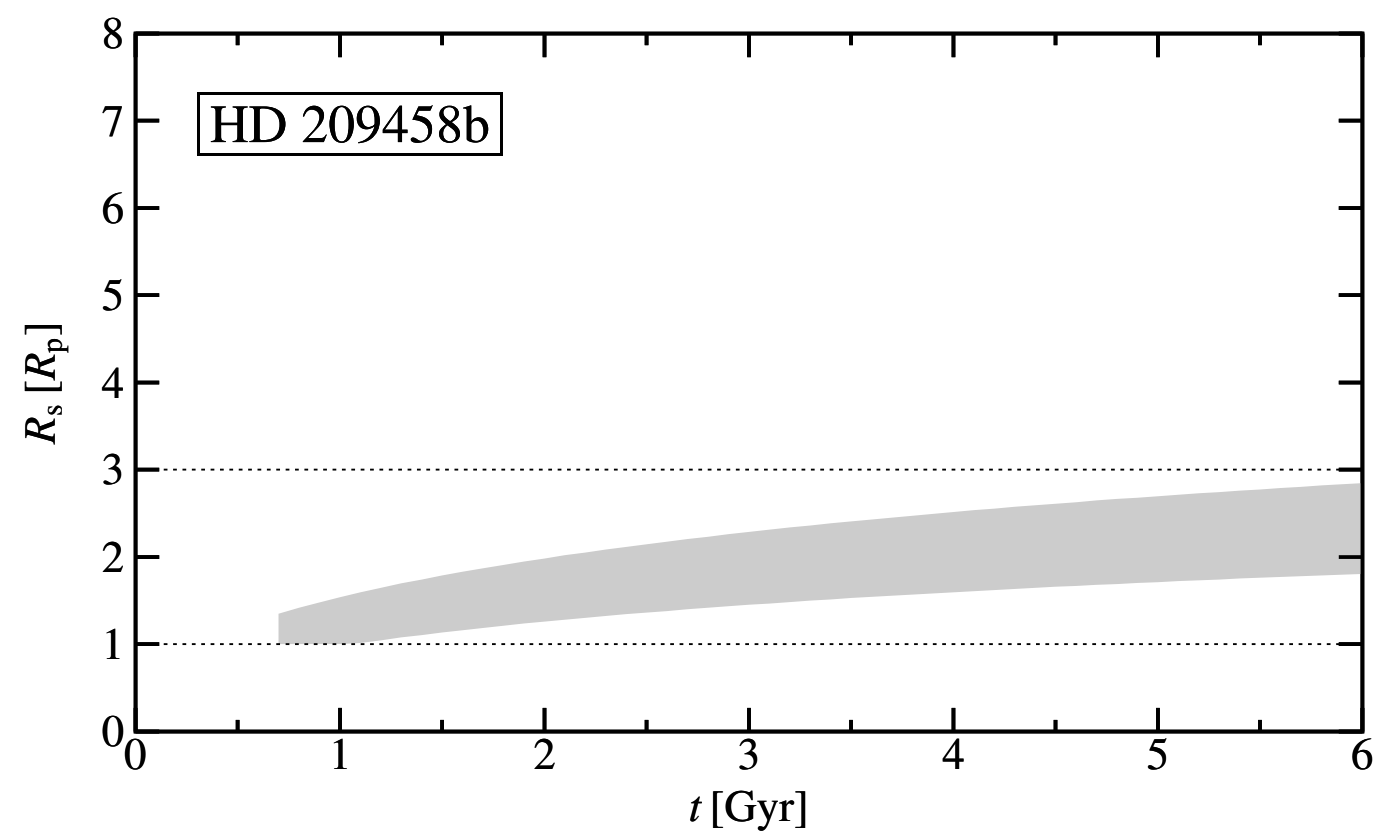

Figure 5.7: Shaded area: Estimated magnetospheric standoff distance for the planet HD 209458b as a function of stellar age, given in planetary radii. Dotted lines: size of the planet, and size of the expanded upper atmosphere (see text).

well protected against such an increased atmospheric mass loss, the planet HD 209458b is likely to have experienced this effect during its complete lifetime. 


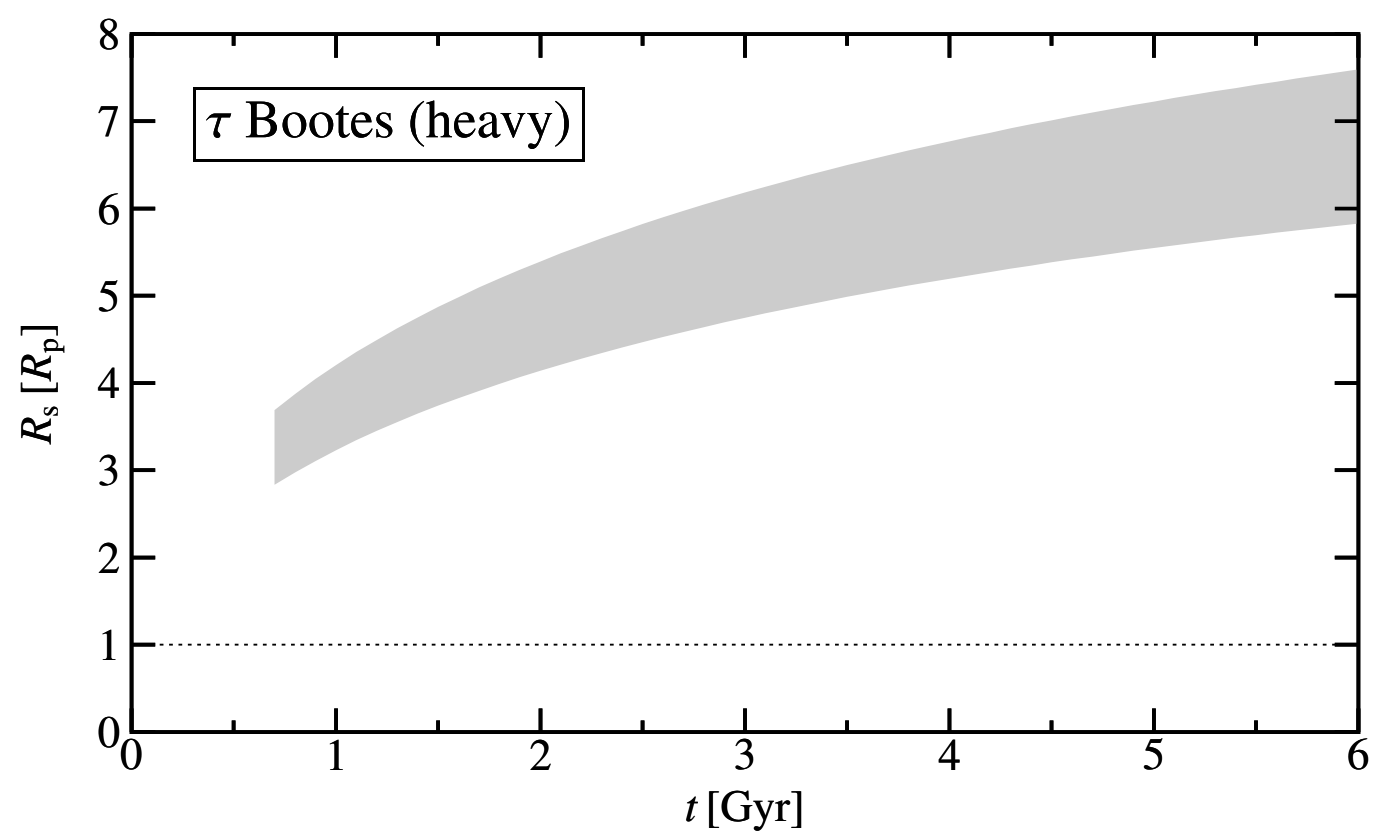

Figure 5.8: Shaded area: Estimated magnetospheric standoff distance for the planet $\tau$ Bootes $\mathrm{b}$ as a function of stellar age, given in planetary radii. Dotted line: size of the planet.

\subsubsection{Size of the magnetosphere of terrestrial planets}

In this section, the analysis of Section 5.3.3 is repeated for different classes of terrestrial exoplanets. For an Earth-like planet, values were already presented in Grießmeier et al. (2005b). The other cases are presented here for the first time.

As in Section 5.3.3, the variables $n, v$ and $T$ appearing in eq. (5.48) are taken to be functions of the stellar age (see Section 5.1). All other parameters are kept constant. The stellar parameters (mass and radius) and the orbital distance of the planet are chosen such that the planets are located within the habitable zone (see Section 2.3) of a K/M type star of 0.5 solar masses: $M_{\star}=0.5 M_{\odot}, R_{\star}=0.46 R_{\odot}$, and $d=0.2 \mathrm{AU}$. The magnetic moment $\mathcal{M}$ of the planet is taken from Section 4.6. The standoff distance during a CME encounter is computed with the numbers for $n_{\mathrm{CME}}^{\mathrm{s}}, v_{\mathrm{CME}}$ and $T_{\mathrm{CME}}$ given in Section 5.2 .

Table 5.5 compares the standoff distances resulting from eq. (5.47) for the different configurations. As in Section 5.3.3, results are given for three different stellar ages, namely 4.6 Gyr, 1.0 Gyr, and 0.7 Gyr. The corresponding standoff distances are labelled $R_{\mathrm{s}}^{4.6}, R_{\mathrm{s}}^{1.0}$ and $R_{\mathrm{s}}^{0.7}$, respectively. The standoff distance arising from a planet-CME interaction is denoted by $R_{\mathrm{s}}^{\mathrm{CME}}$.

From Table 5.5, the following results can be obtained:

- The sizes of the magnetospheres for Earth-like and for Mercury-like planets are very different from the values measured for Earth and Mercury in the solar system. This has two reasons: (a) because of tidal locking at $0.2 \mathrm{AU}$, the magnetic moments of the planets shown in Table 5.5 do not correspond to those of Earth and Mercury, and (b) the stellar wind environment at $0.2 \mathrm{AU}$ around a much smaller $\mathrm{K}$ or $\mathrm{M}$ type star is very different from the solar wind environment at Earth and Mercury. 


\begin{tabular}{|l||c|c|c||c|}
\hline \multicolumn{1}{|l||}{ Planet } & $R_{\mathrm{s}}^{4.6}\left[R_{\mathrm{p}}\right]$ & $R_{\mathrm{s}}^{1.0}\left[R_{\mathrm{p}}\right]$ & $R_{\mathrm{s}}^{0.7}\left[R_{\mathrm{p}}\right]$ & $R_{\mathrm{s}}^{\mathrm{CME}}\left[R_{\mathrm{p}}\right]$ \\
\hline \hline Earth-like & $2.18 \ldots 4.12$ & $1.22 \ldots 2.31$ & $1.07 \ldots 2.02$ & $1.13 \ldots 2.14$ \\
\hline Mercury-like $^{a}$ & $2.14 \ldots 10.7$ & $1.19 \ldots 5.98$ & $1.04 \ldots 5.23$ & $1.11 \ldots 5.56$ \\
$\quad$ (locked) $^{a}$ & $2.14 \ldots 5.11$ & $1.19 \ldots 2.85$ & $1.04 \ldots 2.50$ & $1.11 \ldots 2.65$ \\
\hline Large Earth $^{b}$ & $2.36 \ldots 11.1$ & $1.32 \ldots 6.21$ & $1.15 \ldots 5.44$ & $1.22 \ldots 5.77$ \\
$\quad$ (locked) $^{b}$ & $2.36 \ldots 4.15$ & $1.32 \ldots 2.32$ & $1.15 \ldots 2.03$ & $1.22 \ldots 2.16$ \\
\hline Ocean Planet & $1.54 \ldots 2.80$ & $1.0 \ldots 1.56$ & $1.0 \ldots 1.37$ & $1.0 \ldots 1.45$ \\
\hline
\end{tabular}

Table 5.5: Magnetospheric standoff distances for terrestrial exoplanets at $0.2 \mathrm{AU}$ around a K/M star of mass $M_{\star}=0.5 M_{\odot}$ and radius $R_{\star}=0.46 R_{\odot}$, given in planetary radii. Second to fourth column: Standoff distances for terrestrial exoplanets subject to stellar winds of stars with different ages (4.6 Gyr, 1.0 Gyr, 0.7 Gyr). Fifth column: Standoff distances $R_{\mathrm{s}}^{\mathrm{CME}}$ for terrestrial exoplanets subject to stellar coronal mass ejections. Notes: ${ }^{(a)}$ For distances $d \geq 0.17$ AU, Mercury falls into the "potentially locked" regime. Thus, the upper limit for $\mathcal{M}$ is identical to that of the freely rotating case. Values for a Mercury-like planet still tidally locked at $0.2 \mathrm{AU}$ are given for comparison. ${ }^{(b)}$ For distances $d \geq 0.197 \mathrm{AU}$, a Large Earth falls into the "potentially locked" regime. Thus, the upper limit for $\mathcal{M}$ is identical to that of the freely rotating case. Values for a Large Earth still tidally locked at $0.2 \mathrm{AU}$ are given for comparison.

- The dense and fast stellar winds of young stars lead to a considerable compression of the planetary magnetospheres.

- For weakly magnetised planets (like the Ocean Planet, if the lower limit of the magnetic moment estimation is used), the standoff distance can be compressed down to the planetary surface. In this case, a totally different type of interaction will take place.

- For the case of the Large Earth, the larger magnetic moment $\mathcal{M}$ when compared to the Earth-like planet leads to a larger absolute value of $R_{\mathrm{s}}$. This effect is partially compensated by the larger planetary radius, so that when $R_{\mathrm{s}}$ is given in units of planetary radii $R_{\mathrm{p}}$ the results of both planets are similar.

- For all planets in this list, young stellar winds can compress the magnetosphere down to levels of approximately $1.15 R_{\mathrm{p}}$, at least if the planet is weakly magnetised (i.e. if the lower limit for $\mathcal{M}$ applies). For the Earth, this corresponds to an altitude of $1000 \mathrm{~km}$ above the planetary surface. For such small magnetospheres, strongly enhanced atmospheric mass loss is expected (Khodachenko et al. 2006).

- For planets under the influence of stellar coronal mass ejections the compression of the magnetosphere is comparable to the case of the stellar wind of a young star $\left(t_{\star} \approx 1.0 \mathrm{Gyr}\right)$.

For stellar ages different from those given in Table 5.5, the standoff distance of an Earth-like planet can be obtained from Figure 5.9. The area between the minimum and maximum value for $R_{\mathrm{s}}$ is shaded in grey. The dotted lines represent the location of the 


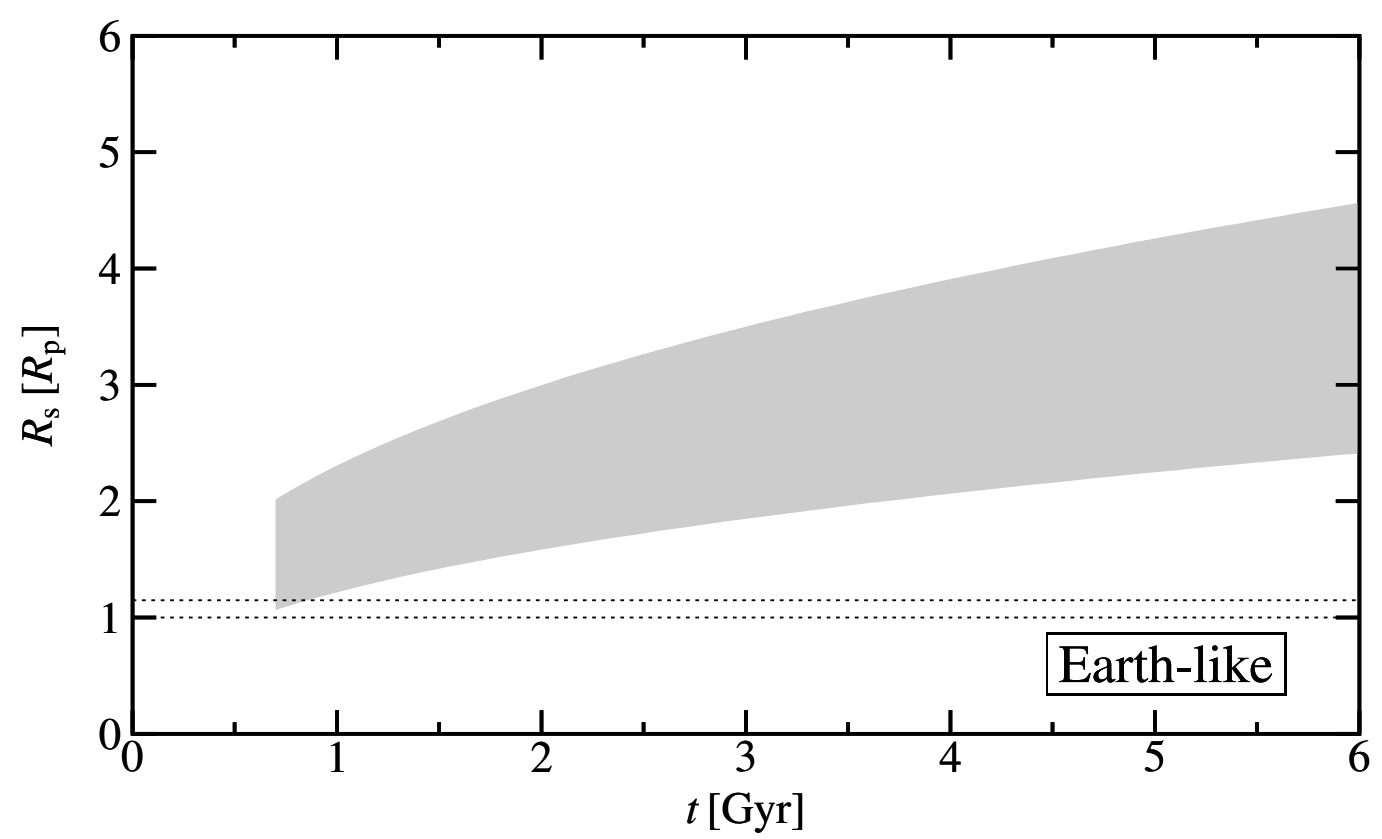

Figure 5.9: Estimated magnetospheric standoff distance for an Earth-like planet at $0.2 \mathrm{AU}$ around a star with mass $M_{\star}=0.5 M_{\odot}$ as a function of stellar age, given in planetary radii. Dotted lines: planetary surface, and distance relevant for atmospheric mass loss (see text).

planetary surface $\left(1.0 R_{\mathrm{p}}\right)$ and the height below which the stellar wind starts to directly act on the planetary atmosphere $\left(1.15 R_{\mathrm{p}}\right)$. For stellar ages $\geq 0.7 \mathrm{Gyr}$, the atmosphere of an Earth-like exoplanet is not strongly affected by the stellar wind.

Similarly to Figure 5.9, Figures 5.10, 5.11 and 5.12 give the standoff distance $R_{\mathrm{s}}$ as a function of the stellar system age $t$. The cases analysed are those of a Mercury-like planet, a "Large Earth" and an "Ocean Planet", respectively. In all three cases, the planet is in an orbit with a semi-major axis of $0.2 \mathrm{AU}$ around a star with a stellar mass of $0.5 M_{\odot}$. Furthermore, tidal locking is assumed. Because for a Mercury-like planet and for a Large Earth this is not certain at the given distance, the standoff distance may be somewhat larger, see Table 5.5. While the atmospheres of a Mercury-like planet and a Large Earth are protected from the stellar wind in a similar way to the Earth-like case of Figure 5.9, a young Ocean Planet experiences strong interaction, and a strongly enhanced atmospheric mass loss rate has to be expected. 


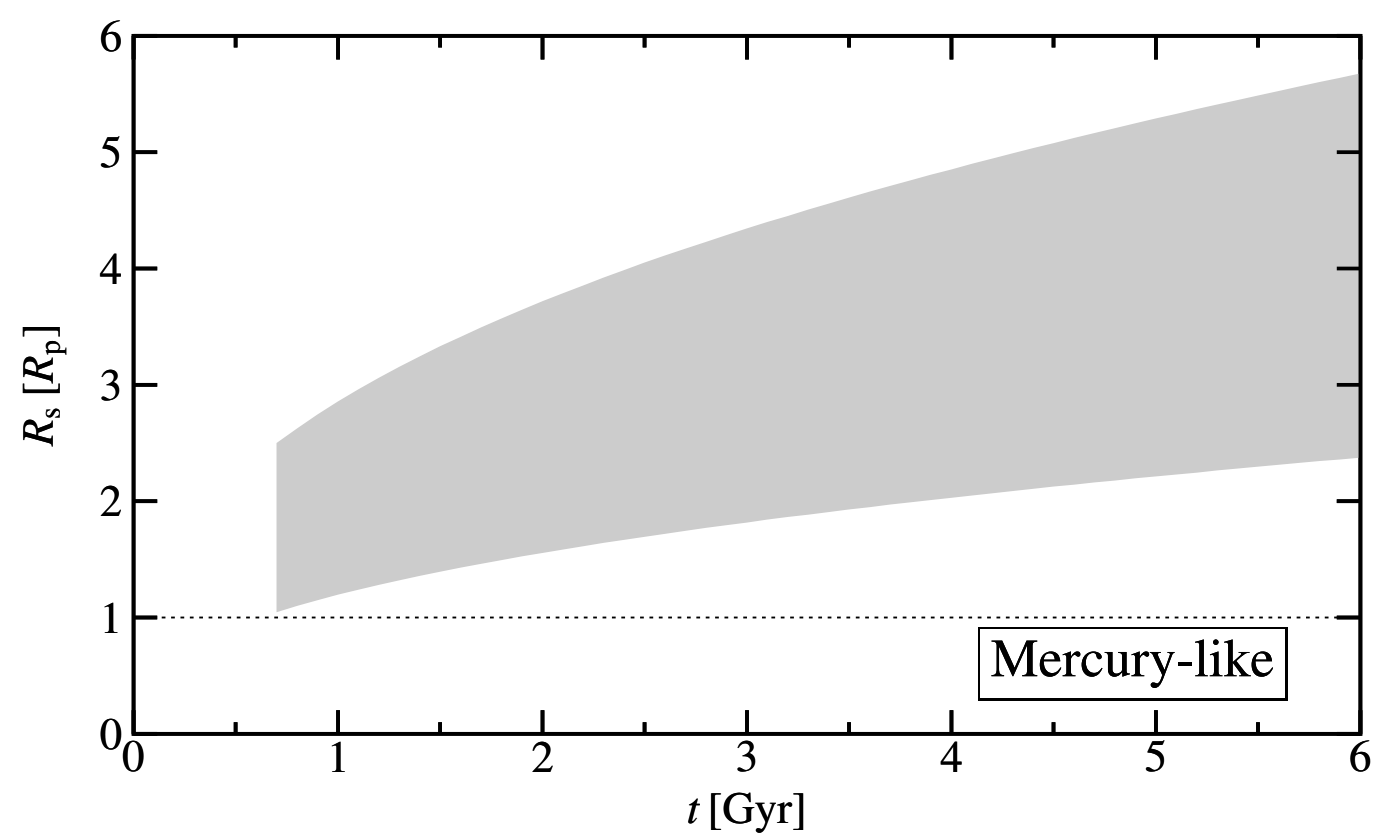

Figure 5.10: Estimated magnetospheric standoff distance for a Mercury-like planet at $0.2 \mathrm{AU}$ around a star with mass $M_{\star}=0.5 M_{\odot}$ as a function of stellar age, given in planetary radii. Dotted line: planetary surface.

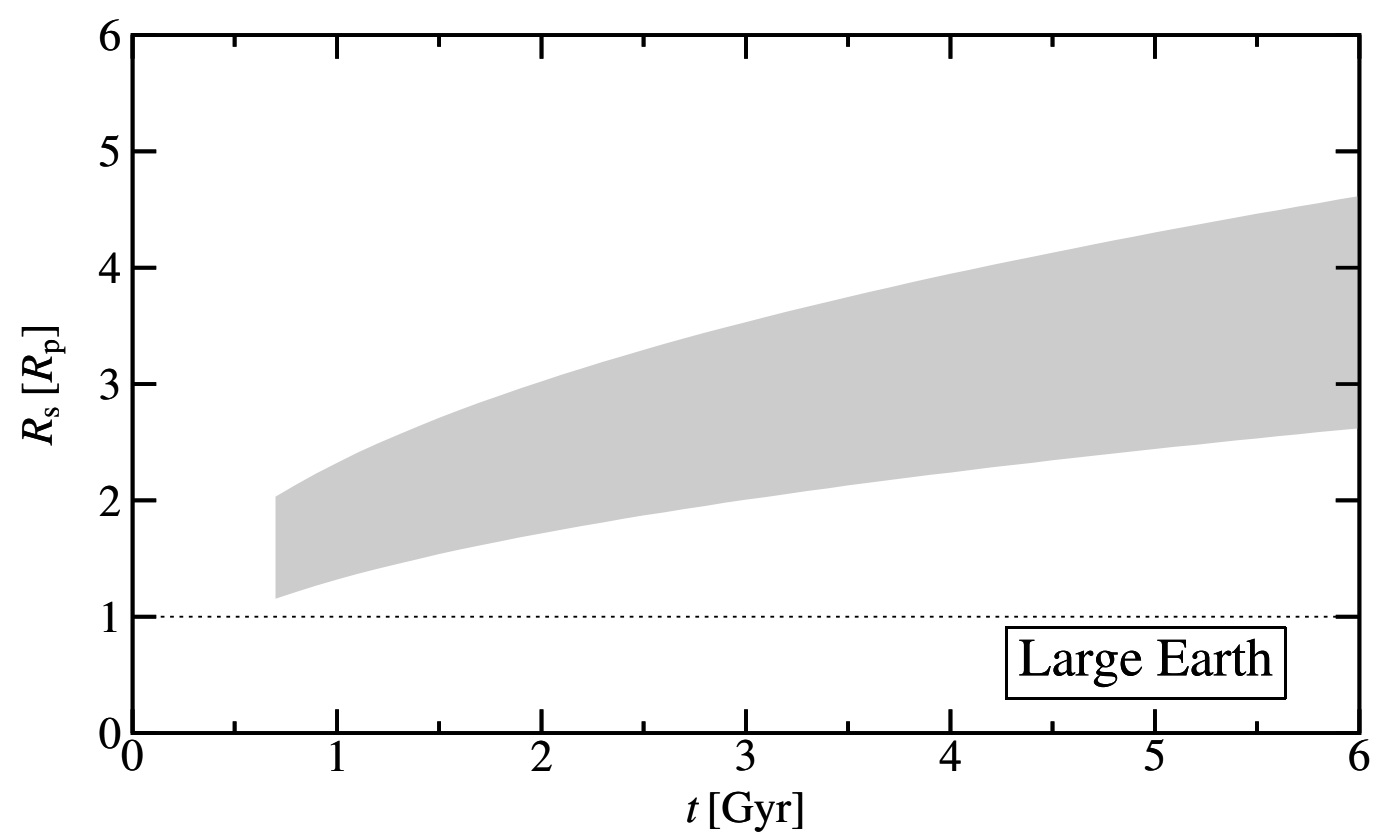

Figure 5.11: Estimated magnetospheric standoff distance for a Large Earth at $0.2 \mathrm{AU}$ around a star with mass $M_{\star}=0.5 M_{\odot}$ as a function of stellar age, given in planetary radii. Dotted line: planetary surface. 


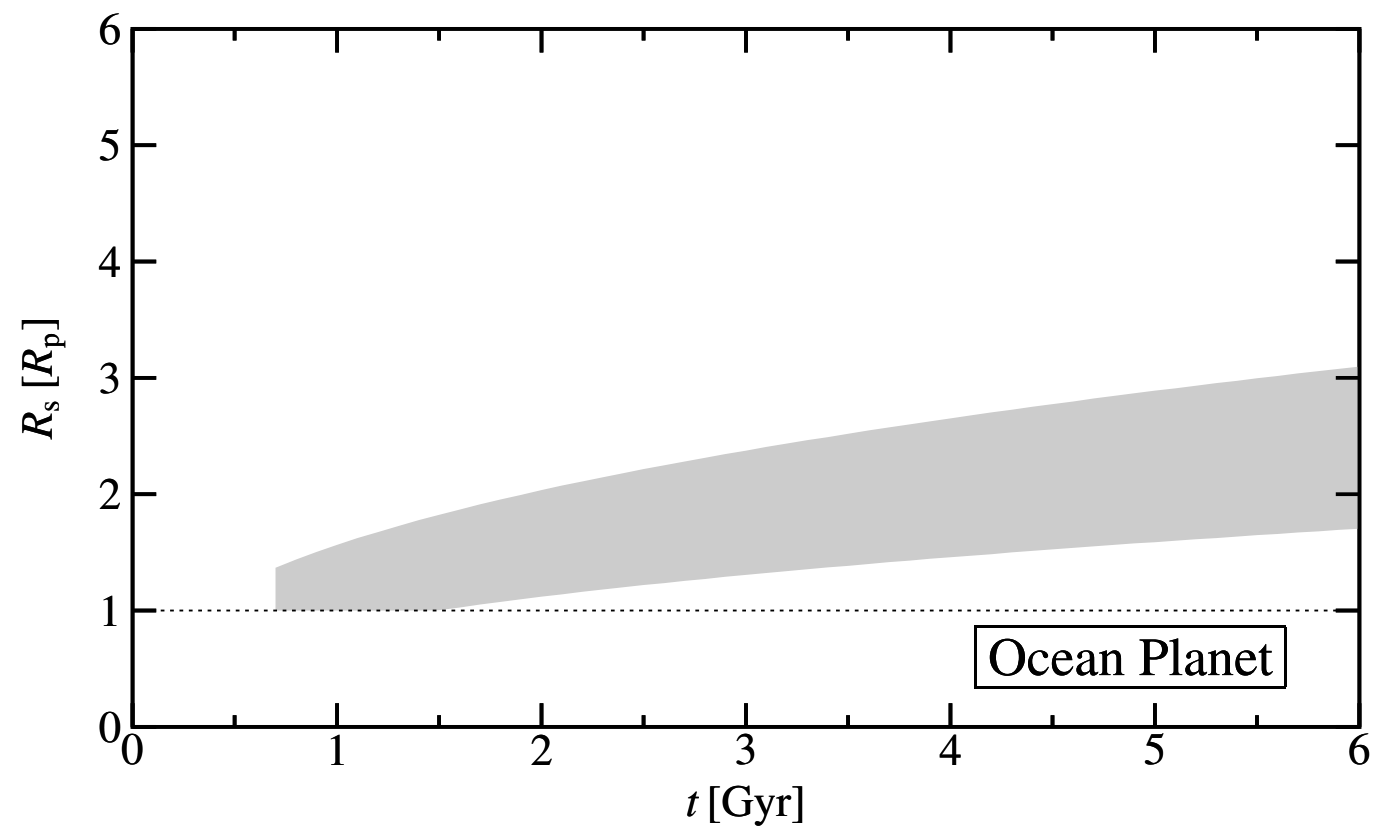

Figure 5.12: Estimated magnetospheric standoff distance for an Ocean Planet at $0.2 \mathrm{AU}$ around a star with mass $M_{\star}=0.5 M_{\odot}$ as a function of stellar age, given in planetary radii. Dotted line: planetary surface. 


\title{
6 Nonthermal radio emission from the magnetospheres of Hot Jupiters
}

\author{
Se non è vero, è molto ben trovato. \\ Giordano Bruno, italian poet and philosopher (1548-1600), \\ Degli Eroici Furori
}

The magnetospheres of magnetised solar system planets are known to be sources of intense nonthermal radio emission. Although the exact process is not yet entirely understood, it is widely believed that the nonthermal radio emission is generated by the socalled cyclotron-maser-instability (CMI). See Carr et al. (1983), Zarka (1992) and Zarka (1998) for reviews on planetary radio emission in the solar system. The CMI is described, for example, by Wu and Lee (1979), Zarka (1998), and Ergun et al. (2000).

Similar radio emission is expected for giant exoplanets, especially for Hot Jupiters, where the interaction of the planet with the stellar wind is much stronger than for planets at larger orbital distances (Zarka et al. 1997, Farrell et al. 1999, Zarka et al. 2001, Lazio et al. 2004, Farrell et al. 2004, Stevens 2005, Grießmeier et al. 2005a, 2006b). For this reason, it is interesting to study whether such extrasolar planetary radio emission could be detected on Earth. Radio detection could yield a wealth of additional information about the emitting planet, including the following:

- The maximum frequency of the radio emission ("cutoff frequency") contains information on the planetary magnetic field. With measurements at different frequencies, the planetary magnetic field (and thus its magnetic moment) could be derived.

- As was suggested, for example by Burke (1992), the radio signal should contain a periodic modulation with the period of the planetary rotation. In first order approximation, Hot Jupiters can be assumed to be tidally locked, so that the rotation period is equal to the orbital period. Thermal atmospheric tides, however, are expected to lead to a deviation from perfect tidal locking (Showman and Guillot 2002). The influence of this effect could be estimated using the periodicity of a planetary radio signal.

- With high-quality observations it could also be possible to obtain information on the existence of satellites around the planet (Stevens, 2005). Hot Jupiters $(d \lesssim 0.05$ $\mathrm{AU})$, however, can only have satellites of very low masses $\left(\lesssim 10^{-4}\right.$ Earth masses for a solar-mass star of 4.6 Gyr age, see Barnes and O'Brien|2002), so that satellites should not be expected in this case. 
- The orbital inclination of the planet could be constrained by studying planetary radio emission (Stevens 2005$)$.

- Finally, with sensitive enough instruments, the measurement of planetary radio emission could be used as an additional method for the detection and confirmation of new exoplanets.

For these reasons, radio emission from Hot Jupiters constitute an active field of research, including both observational efforts and theoretical work aiming at reasonable predictions for different target planets.

It is the aim of Section 6.1 to study how much radio flux can be expected from Hot Jupiters and which parameters are the most important ones (i.e. for which planetary system the expected emission is largest). The second step, after the detection of a signal, would be to identify whether the observed radiation emanates from the star of from the planet. This question is addressed in Section 6.2. Finally, the expected radio flux is compared to the sensitivities of past and future observation campaigns in Section 6.3 .

\subsection{Planetary radio emission}

In this section, the results obtained in previous sections on planetary magnetic moments (Section 4.4), on the stellar wind (Section 5.1), on stellar coronal mass ejections (Section 5.2), and on planetary magnetospheres (Section 5.3.3) are put together to obtain an estimation for the radio flux that can be expected from Hot Jupiters. Because the radio flux estimation is based on the knowledge obtained in the solar system, Section 6.1.1 briefly describes the flux density spectrum of Jupiter and discusses the observed solar wind control of planetary radio emission. In Section 6.1.2 the radio flux expected from different extrasolar planets under present-day stellar wind conditions is calculated. Section 6.1.3 expands this discussion by taking into account the stellar wind evolution with time. It will be shown how this affects the planetary radio emission. This is compared to the radio flux expected from a planet colliding with a stellar CME in Section 6.1.4.

\subsubsection{Planetary radio emission in the solar system}

The first measurement of Jupiter's radio emission (the strongest known planetary radio emission) was made by Burke and Franklin (1955) at a frequency of $22 \mathrm{MHz}$. Due to the Earth's ionosphere, frequencies below 5 to $10 \mathrm{MHz}$ (Zarka et al. 1997) are not accessible for ground-based observations. This is also the reason why radio emission from other planets of the solar system (including the radio emission from the Earth's magnetosphere) were unknown at that time. The full radio spectrum of Jupiter (including the low-frequency component) could only be determined years later by the PRA experiment on both Voyager spacecraft (Zarka 1992). About two days of Voyager data (obtained from a distance of 100-500 planetary radii) were used to compute the spectrum, which was first published in 1992. More recently, the spectrum was recalculated with much more accuracy using six months of Cassini-RPWS data (Zarka et al.2004). Fig. 6.1 is based on that spectrum. Unfortunately, Cassini-RPWS data are only available for $f \leq 16.1 \mathrm{MHz}$. For 


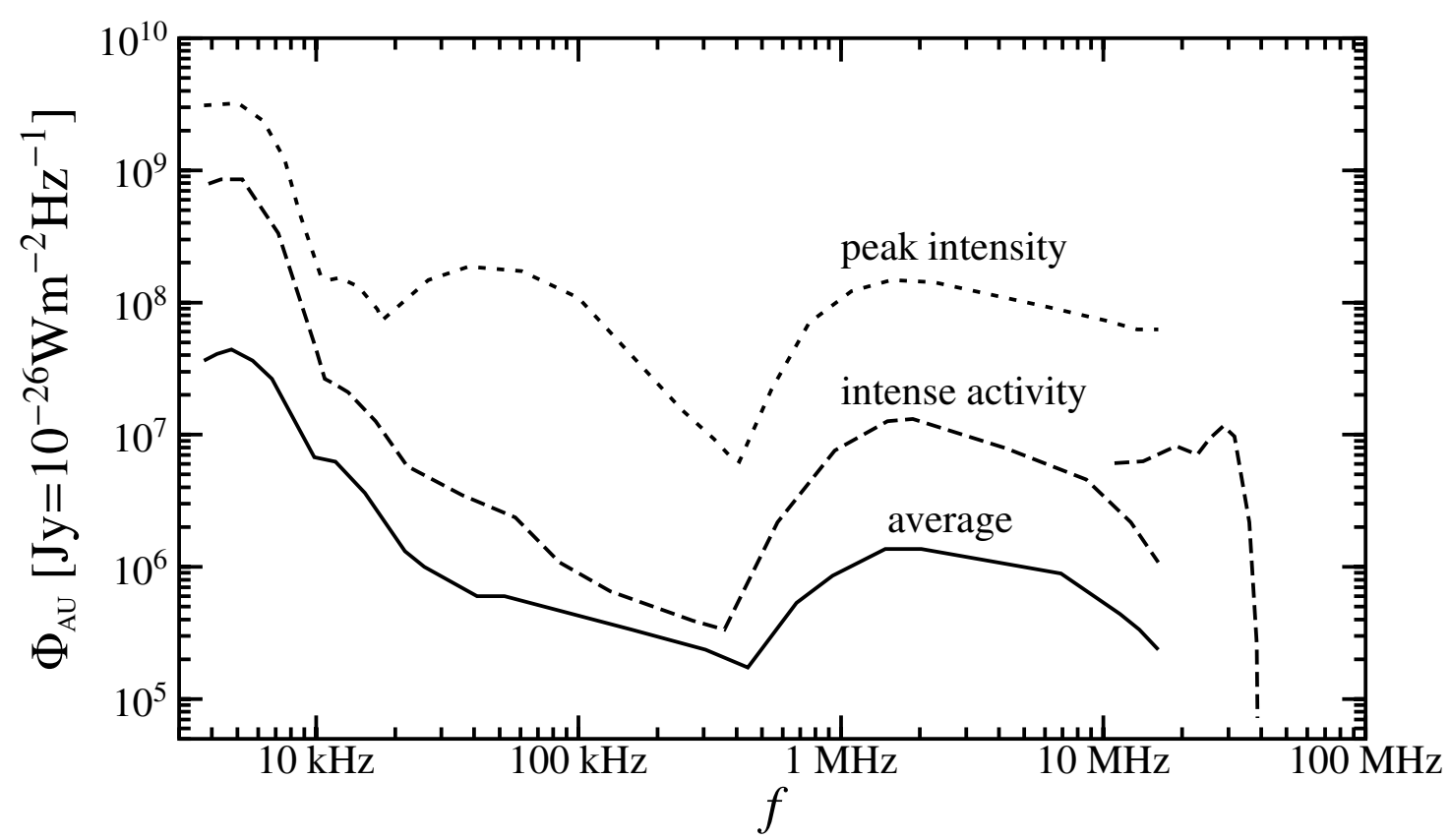

Figure 6.1: Jupiter's radio spectrum as observed at a distance of 1 AU. Solid line: rotation averaged emission. Dashed lines: rotation averaged emission at times of intense activity. Dotted line: peak intensities during active periods. For $f \leq 16.1 \mathrm{MHz}$, the data were obtained by the Cassini RPWS instrument (Zarka et al.|2004). The high-frequency data are taken from Zarka et al.(1995).

higher frequencies, spectral data from Zarka et al. (1995) are shown, corresponding to periods of intense activity (Zarka et al.|2004). It can be seen that the peak flux densities can be up to 100 times the averaged values. The observed spectrum is highly time-dependent, e.g. through solar wind variability (see below), and also depends on the observer's position (due to beaming effects). To facilitate the comparison with exoplanetary and solar radio emission, the flux densities shown in Figure 6.1 are taken at a distance of 1 AU.

The high frequency cutoff in the spectrum shown in Fig. 6.1 (dashed line) can be explained as follows. The radio emission is produced close to the local electron gyrofrequency along auroral fieldlines. Thus, the highest frequency emission will be generated at the location with the strongest magnetic field, i.e. closest to the planetary surface. This yields the "high-frequency cutoff" of about $40 \mathrm{MHz}$ in Fig. 6.1. A more complete discussion of the different components of Jupiter's radiation can be found in Zarka (1998), updated in Zarka et al. (2004). Note that planetary radio emission is strongly circularly polarised (Zarka 1992, 1998).

In the solar system, it is known that planetary radio emission is driven by the stellar wind. The power emitted in the Earth's auroral kilometric radiation (AKR), Saturn's kilometric radiation (SKR) and Jupiter's hectometric emission (HOM) were shown to be strongly correlated with solar wind parameters, e.g., by Gallagher and D'Angelo (1981), Desch and Rucker (1983) and Desch and Barrow (1984), respectively. Later, various publications extended these studies, finding, for example, a similar, albeit somewhat weaker correlation for other components of Jupiter's radiation and for some components of Uranus' and Neptune's radiation. An overview is given in the review papers of Rucker (1987) and Zarka (1998). Of special interest to this work is the good correlation of the 
radio power with the solar wind dynamic pressure $n v^{2}$ and with the solar wind kinetic energy flux $n v^{3}$. Such a correlation was found, for example, by Desch and Rucker (1983) and by Barrow and Desch (1989), for SKR and HOM, respectively, and will be the starting point for the radio flux model in Section 6.1.2.

At the same time it is interesting to note that interplanetary shocks created by CMEs are known to trigger strong decametric (DAM) and hectometric (HOM) emission on Jupiter. For example, Prangé et al. (1993) found indications that an event of very strong DAM-emission (at a level observed only a few times every year) was triggered by a solar wind disturbance created by a CME. Similarly, Gurnett et al. (2002) found strong HOM radiation triggered by an interplanetary shock observed by the spacecraft Cassini and Galileo. The peak of emitted intensity occurred at the time when the solar wind density reached its maximum. The question of increased planetary radio emission triggered by CMEs is taken up in Section 6.1.4.

\subsubsection{Model of exoplanetary radio emission}

The radio emission of a Hot Jupiter is expected to differ considerably from Jupiter's radio emission for several reasons. First, because of tidal locking (Section 3.1.4), its magnetic moment is strongly reduced (Section 4.4). Second, the proximity to the star leads to a totally different stellar wind environment with sub-Alfvénic stellar wind velocities and strongly enhanced stellar wind densities. Both these effects lead to a strong compression of the magnetosphere (Section 5.3.3), but at the same time the increased stellar wind flux is also responsible for a higher energy flux into the magnetosphere. All these effects have an influence on the radio power emitted from a Hot Jupiter planet, as will be shown in the following. The discussion in this section is based on the works of Grießmeier et al. (2005a) and Grießmeier et al. (2006c). Both the stellar wind model of Section 5.1 and the improved pressure equilibrium of Section 5.3.2 are taken into account. The importance of a distance-dependent stellar wind model for the calculation of planetary radio emission was first shown by Grießmeier et al. (2006c).

A simple way to estimate the total emitted radio power of planets in the solar system was originally suggested by Desch and Kaiser (1984). This model was used to estimate the radio flux of the planets Uranus (Desch and Kaiser 1984) and Neptune (Desch 1988). Later, it was applied to predict the radio emission from extrasolar planets (Farrell et al. 1999). The argumentation is as follows: The total power emitted in the radio range $P_{\text {rad }}$ is assumed to be roughly proportional to the total power incident $P_{\text {input }}$ on the magnetosphere (Zarka et al.2001):

$$
P_{\text {rad }} \propto P_{\text {input }}
$$

with a very small constant of proportionality (of the order of $10^{-6}$ to $10^{-5}$, see e.g. Desch and Kaiser 1984, Zarka 1998, Rucker 2002). The power source $P_{\text {input }}$ is believed to be either the kinetic energy flux of the solar wind protons (Desch and Kaiser 1984, Zarka et al. 1997, Farrell et al. 1999, Zarka et al. 2001, Farrell et al. 2004, Lazio et al. 2004, Stevens 2005, Grießmeier et al. 2005a) or the magnetic energy flux of the interplanetary magnetic field (Zarka et al. 2001, Farrell et al. 2004). The magnetic energy model depends on the component of the magnetic field perpendicular to the flow velocity (Zarka 2004). As discussed in Section 5.3.2.2 the interplanetary magnetic field and the stellar 
wind velocity are almost parallel. For this reason, in this work the kinetic energy flux is assumed to be responsible for planetary radio emission.

The energy input into the magnetosphere is proportional to the cross-section of the magnetosphere as seen by the stellar wind, which is determined by the magnetospheric radius $R_{\mathrm{M}}$ :

$$
P_{\text {input }}=\epsilon \frac{\pi}{2} m R_{\mathrm{M}}^{2} n v_{\mathrm{eff}}^{3},
$$

where $m$ is the mass of the stellar wind protons, $v_{\text {eff }}$ is their effective bulk velocity determined by eq. (5.15), and $n$ is their number density at a distance $d$ from the central star. As most of the protons are deflected around the magnetosphere and only a certain portion of the total solar wind kinetic energy is transferred to the magnetosphere, an additional proportionality constant $\epsilon$ is introduced. If magnetic reconnection is considered to be the dominant energy input mechanism, then one finds $\epsilon \approx 1 / 5$ for Earth (Hill 1979). In the following estimation, $\epsilon$ will be considered to be the same for all planets, and the planetary radio power will be calculated relative to that of Jupiter. For this reason, the value of $\epsilon$ is not required.

The magnetospheric radius is estimated from the magnetopause standoff distance. As described in Section 5.3.1.

$$
R_{\mathrm{M}} \approx 2 R_{\mathrm{s}} .
$$

In Section 5.3.2 it was shown that the standoff distance is determined from the pressure balance at the substellar point, and that it is given by:

$$
R_{\mathrm{s}}=\left[\frac{\mu_{0} f_{0}^{2} \mathcal{M}^{2}}{8 \pi^{2}\left(m n v_{\mathrm{eff}}^{2}+2 n k_{\mathrm{B}} T\right)}\right]^{1 / 6} .
$$

In those cases where eq. (6.4) yields standoff distances $R_{\mathrm{s}}<R_{\mathrm{p}}$ (i.e. for planets with very weak magnetic moments and/or subject to extremely dense and fast stellar winds of young stars), $R_{\mathrm{s}}$ is set equal to $R_{\mathrm{p}}$.

Inserting equations 6.3 and 6.2 into eq. 6.1), one obtains

$$
P_{\text {rad }} \propto R_{\mathrm{s}}^{2} n v_{\text {eff }}^{3}
$$

This total emitted power is normalised to the radio power emitted by the planet Jupiter, $P_{\text {rad, }}$, so that relation $(6.5)$ becomes

$$
P_{\mathrm{rad}}=\left(\frac{R_{\mathrm{s}} / R_{\mathrm{p}}}{R_{\mathrm{s}, \mathrm{J}} / R_{\mathrm{J}}}\right)^{2}\left(\frac{R_{\mathrm{p}}}{R_{\mathrm{J}}}\right)^{2}\left(\frac{n}{n_{\mathrm{J}}}\right)\left(\frac{v_{\text {eff }}}{v_{\text {eff, }}}\right)^{3} P_{\text {rad }, \mathrm{J}}
$$

In eq. (6.6), $R_{\mathrm{s}}$ was replaced by $R_{\mathrm{s}} / R_{\mathrm{p}} \cdot R_{\mathrm{p}}$ because standoff distances are frequently given in planetary radii $\left(R_{\mathrm{s}} / R_{\mathrm{p}}\right)$. The emitted power, however, depends on the absolute standoff distance $R_{\mathrm{s}}$, see eq. 6.5). $R_{\mathrm{s}}$ has to be either taken from Section 5.3.3 or calculated using eq. (6.4). The values used for Jupiter are: $R_{\mathrm{s}, \mathrm{J}} / R_{\mathrm{J}}=40.1$ (see Section 5.3.3), $R_{\mathrm{J}}=71492$ $\mathrm{km}$ (Cain et al. 1995, note however that usually $R_{\mathrm{p}}$ is already given in Jupiter radii), $n_{\mathrm{J}}=1.98 \cdot 10^{5} \mathrm{~m}^{-3}, v_{\text {eff, }}=523 \mathrm{~km} / \mathrm{s}$. The total radio flux emitted by Jupiter $P_{\text {rad,J }}$ is calculated from Zarka et al. (2004). As suggested by Zarka et al. (2004), the measured value for the decametric contribution was doubled to account for the fact that only part of 


\begin{tabular}{|l|c|}
\hline conditions & $P_{\text {rad,J }}[\mathrm{W}]$ \\
\hline \hline power during average conditions & $0.31 \cdot 10^{11}$ \\
average power during periods of high activity & $2.1 \cdot 10^{11}$ \\
peak power & $11 \cdot 10^{11}$ \\
\hline
\end{tabular}

Table 6.1: Total emitted radio power for Jupiter.

that frequency band was measured. As can be seen in Table 6.1, three different values can be defined. To determine which extrasolar planets are potentially detectable by means of their radio emission, the average radio power during periods of high activity will be used.

The radio power calculated for different Hot Jupiters is given in Table 6.2. Two values are given; the lower radio power corresponds to the small magnetic moment limit, and the higher value is determined by the upper limit for the magnetic moment.

From the total emitted radio power, the radio flux at a given distance can be calculated. Similarly to Farrell et al. (1999), the radio flux is calculated as

$$
\Phi=\frac{P_{\mathrm{rad}}}{\Omega s^{2} \Delta f} .
$$

Here, $\Omega$ is the solid angle of the beam. The distance of the given stellar system from Earth is denoted by $s$, and $\Delta f$ is the emission bandwidth. According to Zarka et al. (2004), one can use $\Omega=1.6$ for the dominating contributions of Jupiter's radio emission. This value will be adopted in the following. It corresponds to a hollow cone with a half-angle aperture of approximately $75^{\circ}$ and with a cone mantle thickness of $15^{\circ}$. The emission bandwidth $\Delta f$ is approximated by

$$
\Delta f=f_{\mathrm{c}}^{\max },
$$

where the maximum cyclotron frequency $f_{\mathrm{c}}^{\max }$ is determined by the maximum magnetic field strength $B_{\mathrm{p}}^{\max }$ close to the polar cloud tops (Farrell et al. 1999),

$$
f_{\mathrm{c}}^{\max }=\frac{e B_{\mathrm{p}}^{\max }}{2 \pi m_{\mathrm{e}}}
$$

where $e$ is the elementary charge, and $m_{\mathrm{e}}$ is the mass of the electron. A planet with a strong magnetic field is a more powerful radio emitter than a planet with a weak field, but it emits in a much broader frequency band. Similarly to eq. (5.43), the polar magnetic field strength can be expressed by

$$
B_{\mathrm{p}}^{\max }=\frac{\mu_{0}}{4 \pi} \frac{2 \mathcal{M}}{R_{\mathrm{p}}^{3}}
$$

\footnotetext{
${ }^{1}$ Frequently, $\Delta f=0.5 f_{\mathrm{c}}^{\max }$ is used for the emission bandwidth. The radio spectra shown in Fig. 6.1 indicate that emission is taking place also for frequencies below $0.5 f_{\mathrm{c}}^{\mathrm{max}}$, so that eq. 6.8 seems more appropriate.
} 
The difference between eq. (5.43) and eq. (6.10) is due to the fact that for a dipole, the polar field strength is twice the equatorial field strength. Inserting eq. (6.8), eq. (6.9) and eq. 6.10) into eq. 6.7) yields

$$
\Phi=\frac{4 \pi^{2} m_{\mathrm{e}} R_{\mathrm{p}}^{3} P_{\mathrm{rad}}}{e \mu_{0} \Omega s^{2} \mathcal{M}}
$$

According to eq. 6.11), a planet with a small magnetic moment (at fixed emitted power $P_{\text {rad }}$ ) and large radius (at fixed magnetic moment $\mathcal{M}$ ) will lead to the highest detectable radio flux at a given distance $s$. In addition, $P_{\text {rad }}$ is large for a planet close to a star with a dense and fast stellar wind. Apparently, planets for which a strong magnetic moment is expected are not automatically the best candidates concerning the search for radio emission. On the other hand, for a weakly magnetised planet, $f_{\mathrm{c}}^{\max }$ will be small, making ground-based detection impossible by placing the emission below the ionospheric cutoff frequency $\left.\right|^{2}$.

Table 6.2 presents some relevant planetary parameters and the results from eqs. (6.6), (6.9) and (6.11). Some of these quantities are given in units normalised to Jupiter (denoted by subscript J), with $R_{\mathrm{J}}=71492 \mathrm{~km}$ (Cain et al. 1995) and $\mathcal{M}_{\mathrm{J}}=1.56 \cdot 10^{27} \mathrm{Am}^{2}$ (Cain et al. 1995). The flux density $\Phi$ is given in Jansky (1 Jy $\left.=10^{-26} \mathrm{~W} \mathrm{~m}^{-2} \mathrm{~Hz}^{-1}\right)$. For $\tau$ Bootes, the planetary radius is not accessible to measurements. Because of this uncertainty, the three different cases discussed in Section 2.6.1 are compared. The values for the magnetic dipole moment are taken from Table 4.4. As results, Table 6.2 shows the total emitted radio power $P_{\text {rad }}$, the maximum frequency of the radio emission $f_{\mathrm{c}}^{\max }$, and two values for the radio flux, namely the radio flux $\Phi_{\mathrm{AU}}$ which is taken at $1 \mathrm{AU}$, and the radio flux $\Phi_{s}$ which is expected at Earth (at the distance $s$ ). The former is a measure for the strength of a planetary radio emission, which will be compared to the expected stellar emission in Section 6.2, while the latter value will be opposed to the detection limits of different telescopes in Section 6.3. Because it is important to have frequencies above the ionospheric cutoff limit $(5-10 \mathrm{MHz})$ for ground-based detection, Table 6.2 also gives the maximum emission frequencies. Note that the upper limit for the flux and maximum frequency are given by the lower limit for the magnetic moment and vice versa.

From Table 6.2 it can be seen that for the planets HD 209458b, OGLE-TR-10b, and possibly also for OGLE-TR-111b and TrES-1b, the radio emission takes place at frequencies below the ionospheric cutoff frequency. The reason for this is the weak magnetic field due to synchronous rotation (see Section 4). For the planets OGLE-TR-56b, OGLETR-113b, OGLE-TR-132b, and $\tau$ Bootes, radio emission at frequencies above the cutoff frequency can be expected. The question whether the emitted radio flux is high enough to allow for detection on Earth (i.e. at at the distance $s$ ) will be studied in Section 6.3.

Comparing the results for $\tau$ Bootes from Table 6.2 with the values given in previous publications, one finds the following:

- The emitted radio power $P_{\text {rad }}$ is smaller by one order of magnitude than that given by Farrell et al.(1999, see Model 2 in their Table 1). This difference is caused by the different magnetic moments used, and by the different treatment of the stellar wind

\footnotetext{
${ }^{2}$ Of course, a space-based radio observatory would avoid the restriction of the frequency range caused by the ionospheric cutoff.
} 


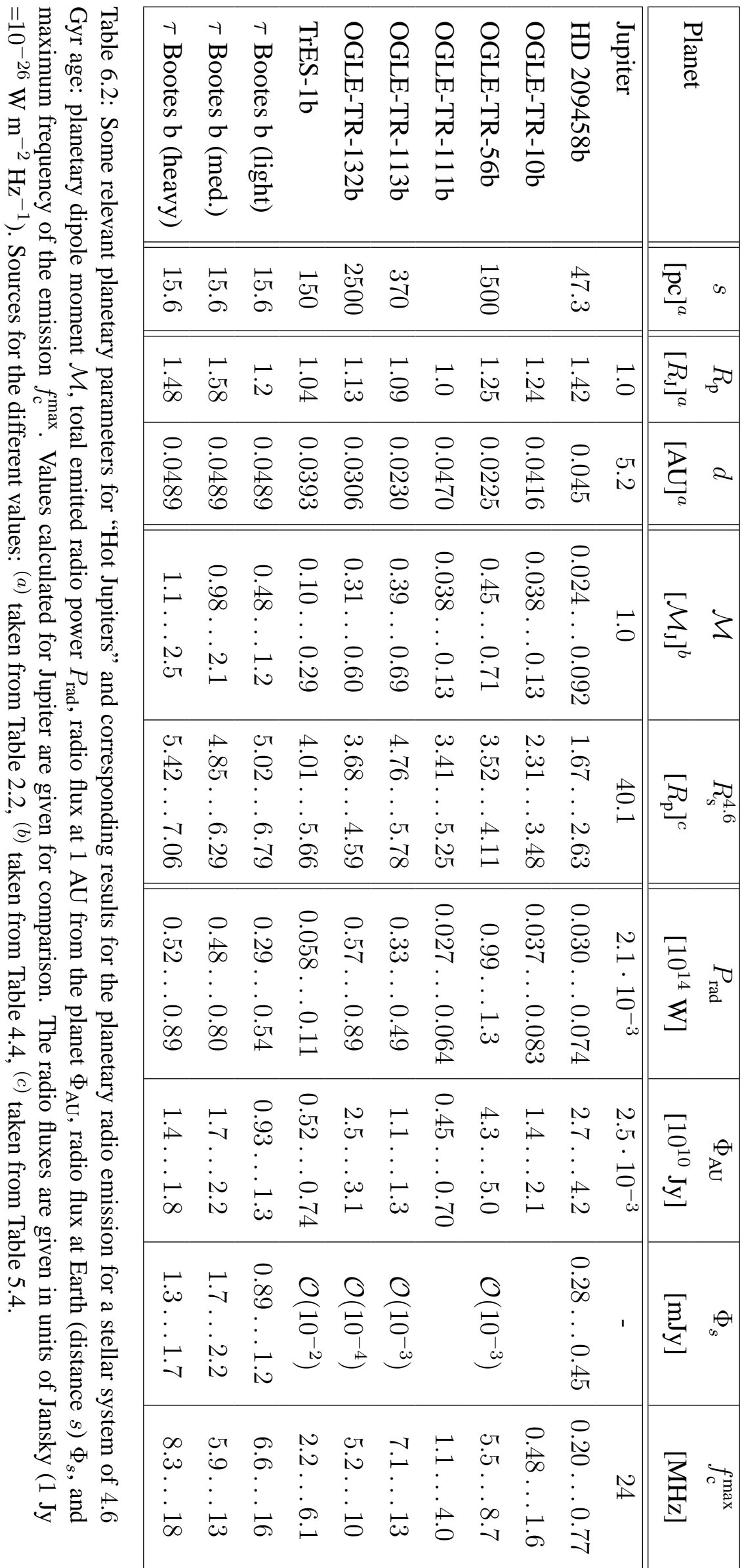


(i.e. Parker model vs. constant stellar wind velocity). When comparing the results of Table 6.2 to those of Lazio et al. (2004), one has to note that Table I of that work gives the peak power, while the results in Table 6.2 were obtained using the average power during periods of high activity. Similarly to Farrell et al. (1999), Lazio et al. (2004) assume that the peak power caused by variations of the stellar wind velocity is two orders of magnitude higher than the average power. However, with the values given in Table 6.1 (obtained from the more recent radio spectra of Zarka et al.|2004), the values Farrell et al. (1999) use for average conditions correspond to periods of high activity, which are less than one order of magnitude below the peak power. During periods of peak emission, the value given in Table 6.2 would be increased by the same factor (i.e. by less than one order of magnitude). For this reason, the peak radio power and the peak radio flux are considerably overestimated in these studies. The radio power given in Table 6.2 is lower than the estimate of Grießmeier et al. (2005a). Three main reasons are responsible for this difference. First, as was explained in Section 4.3, the estimation for the planetary magnetic dipole moment was improved, e.g. by obtaining the size of the dynamo region from the LaneEmden equation (4.50). Second, as was first done by Grießmeier et al. (2006c), a stellar wind model was used which includes the low stellar wind velocities at closein distances (Section 5.1). Third, the pressure equilibrium, from which the size of the magnetosphere is determined, was improved by adding the contributions of the planetary orbital velocity and of the thermal plasma pressure. While for the change in the magnetic moment there is no systematical trend, the distance-dependence of the stellar wind velocity systematically decreases the expected radio power $P_{\text {rad }}$ and thus also the radio flux $\Phi$.

- For the anticipated radio flux $\Phi_{s}$ Farrell et al. (1999. Table 1, Model 2) give values similar to those of the current study. The difference in radio power is not directly reflected here, because different values are used for the magnetic moment $\mathcal{M}$, the solid angle of the emission $\Omega$, and the emission bandwidth $\Delta f$. These differences compensate each other, so that for average conditions the values for the radio flux are approximately comparable. The radio flux given by Lazio et al. (2004) is based on the very high value of the peak power. If it is reduced by a factor 100 to obtain the value for the average emission, the remaining difference to $\Phi$ from Table 6.2 is due to the higher maximum emission frequency (see next item). The differences in radio flux when comparing to Grießmeier et al. (2005a) is caused by the increased emission bandwidth $\Delta f$ and by the improved estimation of the radio power (explained above). Note that the use of a stellar wind model without distance dependence leads to an overestimation of the radio flux by a factor of approx. 2 (Grießmeier et al.2006c).

- As far as the maximum emission frequency $f_{c}^{\max }$ is concerned, the frequency given by Lazio et al. (2004) is higher by a factor of three because the maximum frequency is calculated using $R_{\mathrm{p}}=R_{\mathrm{J}}$ in that work. For planets heavier than $\tau$ Bootes, the magnetic moments are strongly overestimated by Farrell et al. (1999) and by Lazio et al. (2004). This is due to the fact that these works rely on the magnetic moment scaling law of Blackett (1947), which has a large exponent in $r_{\mathrm{c}}$. In addition, these works make use of the mass-radius relation $R_{\mathrm{p}} \propto M_{\mathrm{p}}^{1 / 3}$. Especially for planets with 
large masses like $\tau$ Bootes, this yields unrealistically large planetary radii, magnetic moments (for which a few values are compared in Section 4.4), and emission frequencies. Note that a good estimation of the emission frequency is particularly important, because a difference of a factor of a few can make the difference between radiosignals above and below the ionospheric cutoff frequency.

In order to find more simplified expressions for eqs. (6.6) and (6.11), the limits $k_{\mathrm{B}} T \ll$ $m v_{\text {eff }}^{2}$ and $v_{\text {eff }} \approx v$ are taken. Then, one finds $R_{\mathrm{s}} \propto \mathcal{M}^{1 / 3} n^{-1 / 6} v^{-1 / 3}$, which can be inserted into eq. (6.5). This results in

$$
P_{\text {rad }} \propto R_{\mathrm{s}}^{2} n v_{\text {eff }}^{3} \propto \mathcal{M}^{2 / 3} n^{2 / 3} v^{7 / 3}
$$

Frequently, the stellar wind velocity $v$ is assumed to be independent of the orbital distance, so that the density falls off quadratically: $n=n_{0}\left(d_{0} / d\right)^{2}$, where $n_{0}=n\left(d=d_{0}\right)$. This assumption (which is not well satisfied for close-in planets, see Section 5.1) leads to a further simplification of eq. 6.12):

$$
P_{\text {rad }} \propto \mathcal{M}^{2 / 3} n_{0}^{2 / 3} v^{7 / 3} d^{-4 / 3}
$$

For the radio flux given by eq. 6.11, this means that

$$
\Phi \propto R_{\mathrm{p}}^{3} \mathcal{M}^{-1 / 3} n_{0}^{2 / 3} v^{7 / 3} d^{-4 / 3} s^{-2} .
$$

While both eqs. (6.13) and (6.14) are not as precise as eqs. (6.6) and (6.7), they are useful to discuss the influence of the different parameters. The emitted radio power $P_{\text {rad }}$ increases with increasing magnetic moment, stellar mass loss (which determines $n_{0}$ ) and stellar wind velocity, and with decreasing orbital distance (which is responsible for the stellar wind density at the location of the planet). As described in Section 4, the size of the magnetic moment depends on the rotation state of the planet, which, in turn, is limited by tidal locking (Section 3.1). The reason why $P_{\text {rad }}$ increases with increasing $n_{0}$ and $v$ is that the higher kinetic energy flux dominates over the magnetospheric compression (i.e. the smaller cross-section of the magnetosphere). The radio flux $\Phi$ increases with increasing planetary size, stellar mass loss and stellar wind velocity. It also increases with decreasing magnetic moment, orbital distance and distance to the detector. The decreasing value of $\Phi$ with increasing $\mathcal{M}$ is due to the fact that while $P_{\text {rad }}$ increases, the emission bandwidth $\Delta f$ increases with a higher exponent. Hence, a not too high value of $\mathcal{M}$ is favourable for detection. On the other hand, for a planet with a very small value of $\mathcal{M}$, the maximum emission frequency $f_{\mathrm{c}}^{\max }$ is small, making ground-based detection impossible by placing the emission frequency below the ionospheric cutoff frequency.

So far, stellar wind conditions corresponding to a stellar system age of 4.6 Gyr were compared. The real stellar age will be taken into account in the following section.

\subsubsection{Influence of the stellar system age}

As was shown in Section 5.1.2, the stellar wind velocity of a young star (1 Gyr after reaching the main sequence) is about twice as high as the velocity of today's solar wind (at 4.6 Gyr). Likewise, the density of the stellar wind of a young $\mathrm{G}$ or $\mathrm{K}$ star is about one order of magnitude higher. This has two effects: first of all, it compresses the magnetosphere, 
6.1 Planetary radio emission

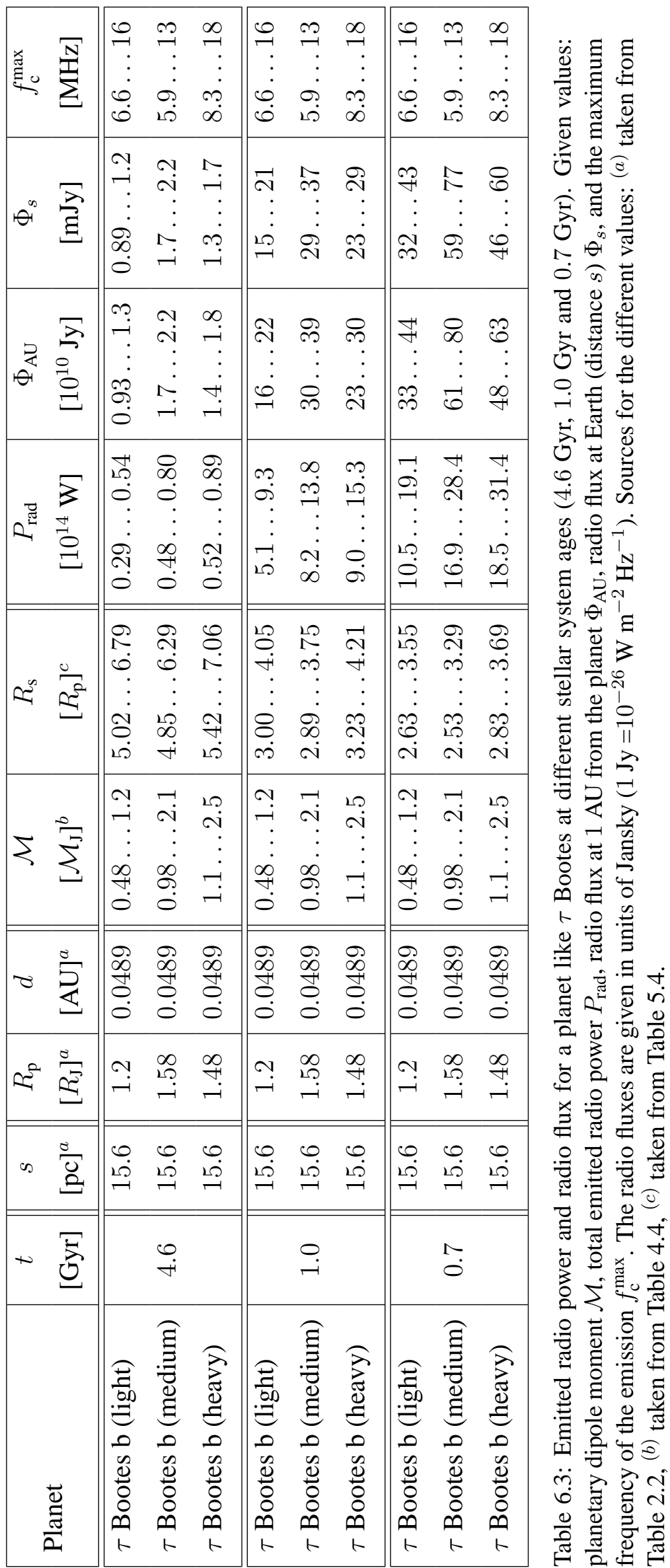




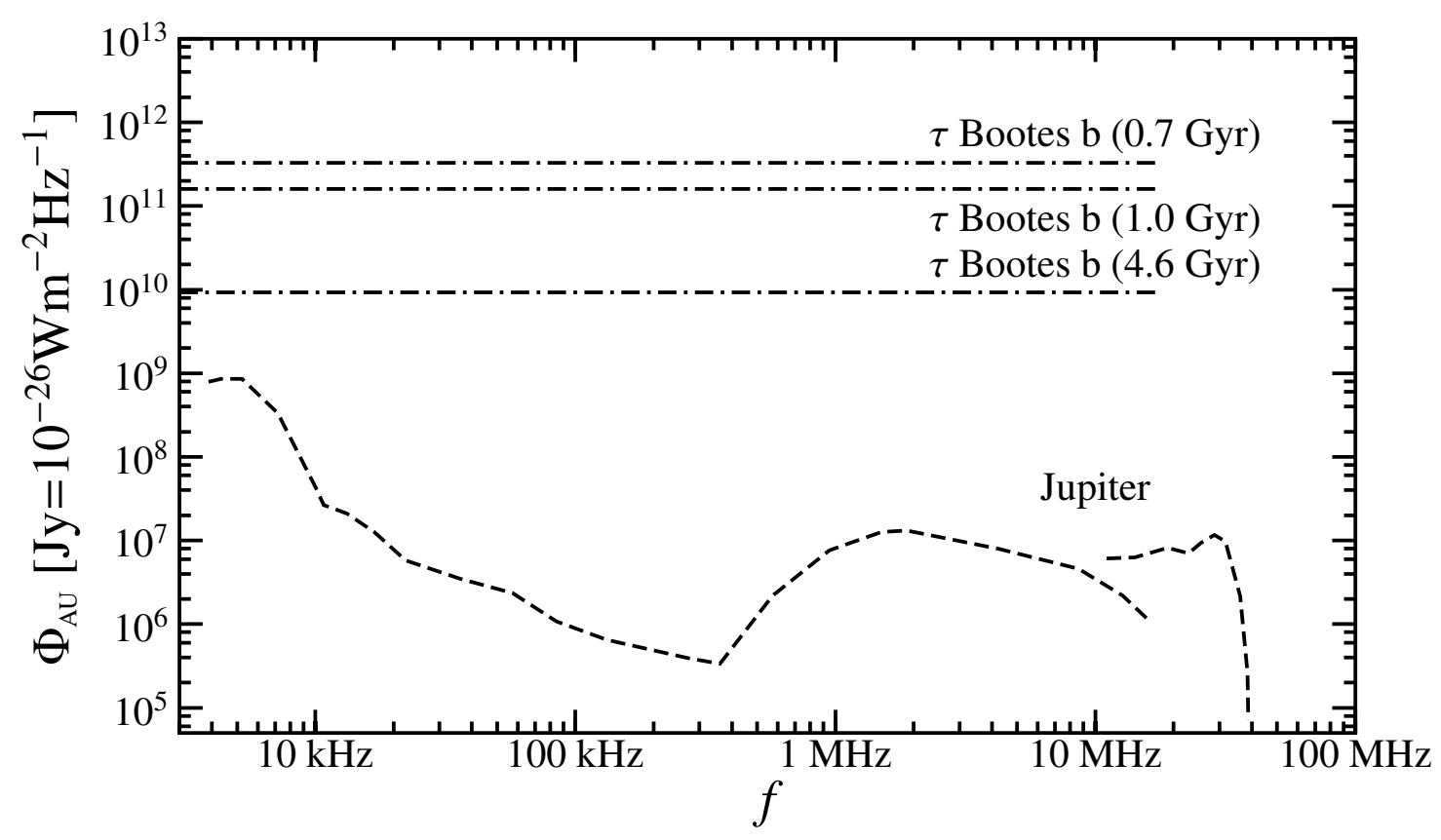

Figure 6.2: Comparison of the radio flux measured from Jupiter (see Fig. 6.1) according to Zarka et al.(1995, 2004) at periods of intense activity (dashed lines) and the lower limit for the radio flux emission from a planet like $\tau$ Bootes $\mathbf{b}$ at different ages (dash-dotted lines). All values are taken at a distance of $1 \mathrm{AU}$.

as can be seen from eq. (5.48). On the other hand, the increased stellar wind parameters also increase the energy input into the magnetosphere, see eq. (6.2). Eq. (6.12) suggests that a denser and faster stellar wind will lead to a stronger planetary radio emission. This effect is important, because the age of extrasolar planet host stars vary, see Table 2.2. To demonstrate this effect, $\tau$ Bootes is chosen as an example, because of all planets in Table 6.2 it is the one with the strongest radio flux at Earth. The stellar wind parameters $v\left(d, t, M_{\star}, R_{\star}\right)$ and $n\left(d, t, M_{\star}, R_{\star}\right)$ are obtained according to Section 5.1.4 and inserted into eqs. (6.6) and 6.11). In this way, the total emitted radio power and the expected flux density are calculated for three different stellar system ages (4.6 Gyr, 1.0 Gyr and 0.7 Gyr). The resulting values are compared in Table 6.3. The stellar mass and radius as well as the planetary radius, magnetic moment and semi-major axis are assumed to be constant, so that the time evolution is caused exclusively by the variation of $v, n$, and $T$. Table 6.3 shows clearly that the radio power and the flux density can be much higher for a planet in a young stellar system (one order of magnitude difference and more). Note that the age of $\tau$ Bootes was estimated to be approximately $1 \pm 0.6$ Gyr by Fuhrmann et al. (1998). For this reason, the radio flux to be expected from that planet is considerably higher than the value obtained when using solar system values for the stellar wind.

Figure 6.2 compares the lower limits for the planetary radio flux $\Phi_{\mathrm{AU}}$ (which correspond to the upper limit for the planetary magnetic moment $\mathcal{M}$ ) obtained for different stellar system ages. The upper limit is higher by a factor of two. The dashed line shows the radio spectrum of Jupiter. One can see that the radio flux emitted by the planet $\tau$ Bootes $\mathrm{b}$ (with an age of $1.0 \mathrm{Gyr}$ ) exceeds Jupiter's radio flux by approximately four orders of magnitude. For a planet like $\tau$ Bootes b, but with a stellar system age of $4.6 \mathrm{Gyr}$, 
the expected radio flux is less by over one order of magnitude.

\subsubsection{Influence of stellar CMEs}

So far, the discussion of planetary radio emission has focused on emission caused by the steady state stellar wind, which can be held responsible for the average radio flux. In Section 5.2 it was shown that perturbations of the stellar wind by CMEs are likely to occur very frequently for Hot Jupiters. At the same time, it is known from observations in the solar system that CMEs can trigger very strong radio emission (see references in Section 6.1.1). Such events will change the peak flux of a close-in planet as well as the frequency of its occurrence. For active stars, the CME-dominated peak radio flux may even replace the average radio flux. For this reason, the radio flux expected from extrasolar planets under the influence of CMEs is calculated in this section. This is done by repeating the calculation of Section 6.1.2 with the stellar wind parameters $n$ and $v$ replaced by the values appropriate for a strong $\mathrm{CME}$, namely $n_{\mathrm{CME}}^{\mathrm{s}}$ and $v_{\mathrm{CME}}$. These values were derived in Section 5.2.1. The estimation of the parameters relevant for CMEs is based on the work of Khodachenko et al. (2006). The corresponding radio flux was calculated for the first time in the work of Grießmeier et al. (2006b), upon which this section is based.

As in Section 6.1.2 the power of the radio emission is taken to be proportional to the power input by the kinetic energy of the stellar wind particles. The kinetic energy model is modified for the situation where the energy input is determined by strong CMEs rather than the steady stellar wind. In that case, the total emitted radio power $P_{\text {rad }}$ and the resulting flux density $\Phi_{s}$ are given by:

$$
P_{\text {rad,CME }}=\left(\frac{R_{\mathrm{s}, \mathrm{CME}} / R_{\mathrm{p}}}{R_{\mathrm{s}, \mathrm{J}} / R_{\mathrm{J}}}\right)^{2}\left(\frac{R_{\mathrm{p}}}{R_{\mathrm{J}}}\right)^{2}\left(\frac{n_{\mathrm{CME}}^{\mathrm{s}}}{n_{\mathrm{J}}}\right)\left(\frac{v_{\text {eff,CME }}}{v_{\text {eff, } \mathrm{J}}}\right)^{3} P_{\text {rad, } \mathrm{J}}
$$

and

$$
\Phi=\frac{4 \pi^{2} m_{\mathrm{e}} R_{\mathrm{p}}^{3} P_{\mathrm{rad}, \mathrm{CME}}}{e \mu_{0} \Omega s^{2} \mathcal{M}} .
$$

Here, $R_{\mathrm{s}} / R_{\mathrm{p}}$ is the standoff distance given in planetary radii. This value was determined in Section 5.3.3. The values used for Jupiter are the following: $R_{\mathrm{s}, \mathrm{J}} / R_{\mathrm{J}}=40.1$ (see Section 5.3.3), $R_{\mathrm{J}}=71492 \mathrm{~km}$ (Cain et al. 1995, note, however, that usually $R_{\mathrm{p}}$ is already given in Jupiter radii), $n_{\mathrm{J}}=1.98 \cdot 10^{5} \mathrm{~m}^{-3}, v_{\text {eff,J }}=523 \mathrm{~km} / \mathrm{s}$. For the total radio flux emitted by Jupiter $P_{\text {rad, }}$, the average radio power during periods of high activity will be used $\left(P_{\text {rad,J }}=2.1 \cdot 10^{11} \mathrm{~W}\right.$, see Table 6.1). The values for $n_{\mathrm{CME}}^{\mathrm{s}}$ (the density of a strong $\mathrm{CME}$ ) and $v_{\mathrm{CME}}$ (the CME velocity) are obtained according to Section 5.2.1. Because these values are larger than the stellar wind parameters (Section 5.2.3), more intense radio emission can be expected.

The radio power and flux density are calculated for one typical example, namely the planet around the star $\tau$ Bootes. In Table 6.4 these results are compared to the values that were derived for a similar planet around a non-flaring star in Section 6.1.3. As before, the lower limit for the radio flux corresponds to the larger magnetic moment, and the upper limit is determined by the smaller magnetic moment. For the radio power, it is the other way round, so that the upper limit for the radio power corresponds to the lower 
6 Nonthermal radio emission from the magnetospheres of Hot Jupiters

\begin{tabular}{|c|c|c|c|c|c|}
\hline Planet & $\begin{array}{c}t \\
{[\mathrm{Gyr}]}\end{array}$ & $\begin{array}{c}P_{\mathrm{rad}} \\
{\left[10^{14} \mathrm{~W}\right]}\end{array}$ & $\begin{array}{c}\Phi_{\mathrm{AU}} \\
{\left[10^{10} \mathrm{Jy}\right]}\end{array}$ & $\begin{array}{c}\Phi_{s} \\
{[\mathrm{mJy}]}\end{array}$ & $\begin{array}{c}f_{\mathrm{c}}^{\max } \\
{[\mathrm{MHz}]}\end{array}$ \\
\hline$\tau$ Bootes b (light) & & $0.29 \ldots 0.54$ & $0.93 \ldots 1.3$ & $0.89 \ldots 1.2$ & $6.6 \ldots 16$ \\
\hline$\tau$ Bootes $\mathrm{b}$ (medium) & $4.6^{a}$ & $0.48 \ldots 0.80$ & $1.7 \ldots 2.2$ & $1.7 \ldots 2.2$ & $5.9 \ldots 13$ \\
\hline$\tau$ Bootes b (heavy) & & $0.52 \ldots 0.89$ & $1.4 \ldots 1.8$ & $1.3 \ldots 1.7$ & $8.3 \ldots 18$ \\
\hline$\tau$ Bootes b (light) & & $5.1 \ldots 9.3$ & $16 \ldots 22$ & $15 \ldots 21$ & $6.6 \ldots 16$ \\
\hline$\tau$ Bootes b (medium) & $1.0^{a}$ & $8.2 \ldots 13.8$ & $30 \ldots 39$ & $29 \ldots 37$ & $5.9 \ldots 13$ \\
\hline$\tau$ Bootes b (heavy) & & $9.0 \ldots 15.3$ & $23 \ldots 30$ & $23 \ldots 29$ & $8.3 \ldots 18$ \\
\hline$\tau$ Bootes b (light) & & $10.5 \ldots 19.1$ & $33 \ldots 44$ & $32 \ldots 43$ & $6.6 \ldots 16$ \\
\hline$\tau$ Bootes $\mathrm{b}$ (medium) & $0.7^{a}$ & $16.9 \ldots 28.4$ & $61 \ldots 80$ & $59 \ldots 77$ & $5.9 \ldots 13$ \\
\hline$\tau$ Bootes b (heavy) & & $18.5 \ldots 31.4$ & $48 \ldots 63$ & $46 \ldots 60$ & $8.3 \ldots 18$ \\
\hline$\tau$ Bootes b (light) & & $6.3 \ldots 11.5$ & $20 \ldots 27$ & $19 \ldots 26$ & $6.6 \ldots 16$ \\
\hline$\tau$ Bootes $\mathrm{b}$ (medium) & CME & $10.1 \ldots 17.1$ & $37 \ldots 48$ & $36 \ldots 46$ & $5.9 \ldots 13$ \\
\hline$\tau$ Bootes b (heavy) & & $11.1 \ldots 18.9$ & $29 \ldots 38$ & $28 \ldots 36$ & $8.3 \ldots 18$ \\
\hline
\end{tabular}

Table 6.4: Comparison of radio emission triggered by the stellar wind and by stellar coronal mass ejection. The following values are listed: total emitted radio power $P_{\text {rad }}$, radio flux at $1 \mathrm{AU}$ from the planet $\Phi_{\mathrm{AU}}$, radio flux at Earth (distance $s$ ) $\Phi_{s}$, and the maximum frequency of the emission $f_{\mathrm{c}}^{\max }$. The radio fluxes are given in units of Jansky $\left(1 \mathrm{Jy}=10^{-26} \mathrm{~W} \mathrm{~m}^{-2} \mathrm{~Hz}^{-1}\right)$. Sources for the different values: ${ }^{(a)}$ taken from Table 6.3 .

limit for the radio flux. One finds that the expected radio flux for radio emission driven by CMEs is comparable to planetary radio emission driven by the stellar wind of a young $\operatorname{star}\left(t_{\star} \approx 1.0 \mathrm{Gyr}\right)$. The radio flux connected to a solar-like stellar wind is lower by more than one order of magnitude.

It is instructive to compare the radio flux caused by stellar coronal mass ejections to the simplified stellar wind case with $k_{\mathrm{B}} T \ll m v_{\text {eff }}^{2}, v_{\text {eff }} \approx v$ and $n=n_{0}\left(d_{0} / d\right)^{2}$. In that case, $P_{\text {rad }}$ and $\Phi$ are given by eqs. (6.13) and (6.14) for stellar wind driven radio emission. This corresponds to a distance dependence according to

$$
\Phi \propto P_{\mathrm{rad}} \propto d^{-4 / 3}
$$

For radio emission driven by strong CMEs, the distance dependence of $n_{\mathrm{CME}}^{\mathrm{s}}$ and $v_{\mathrm{CME}}$ is given by eqs. (5.19) and (5.20), respectively. With eq. (6.12), this leads to the following dependence:

$$
\Phi_{\mathrm{CME}}^{\mathrm{s}} \propto P_{\text {rad,CME }}^{\mathrm{s}} \propto d^{-1.99} .
$$

For this reason, the difference between radio emission triggered by the stellar wind and by CMEs is largest for planets at close orbital distances.

In Figure 6.3, the lower limits for the expected planetary radio flux (corresponding to the upper limit for the planetary magnetic moment) are compared for: a) radio emission 


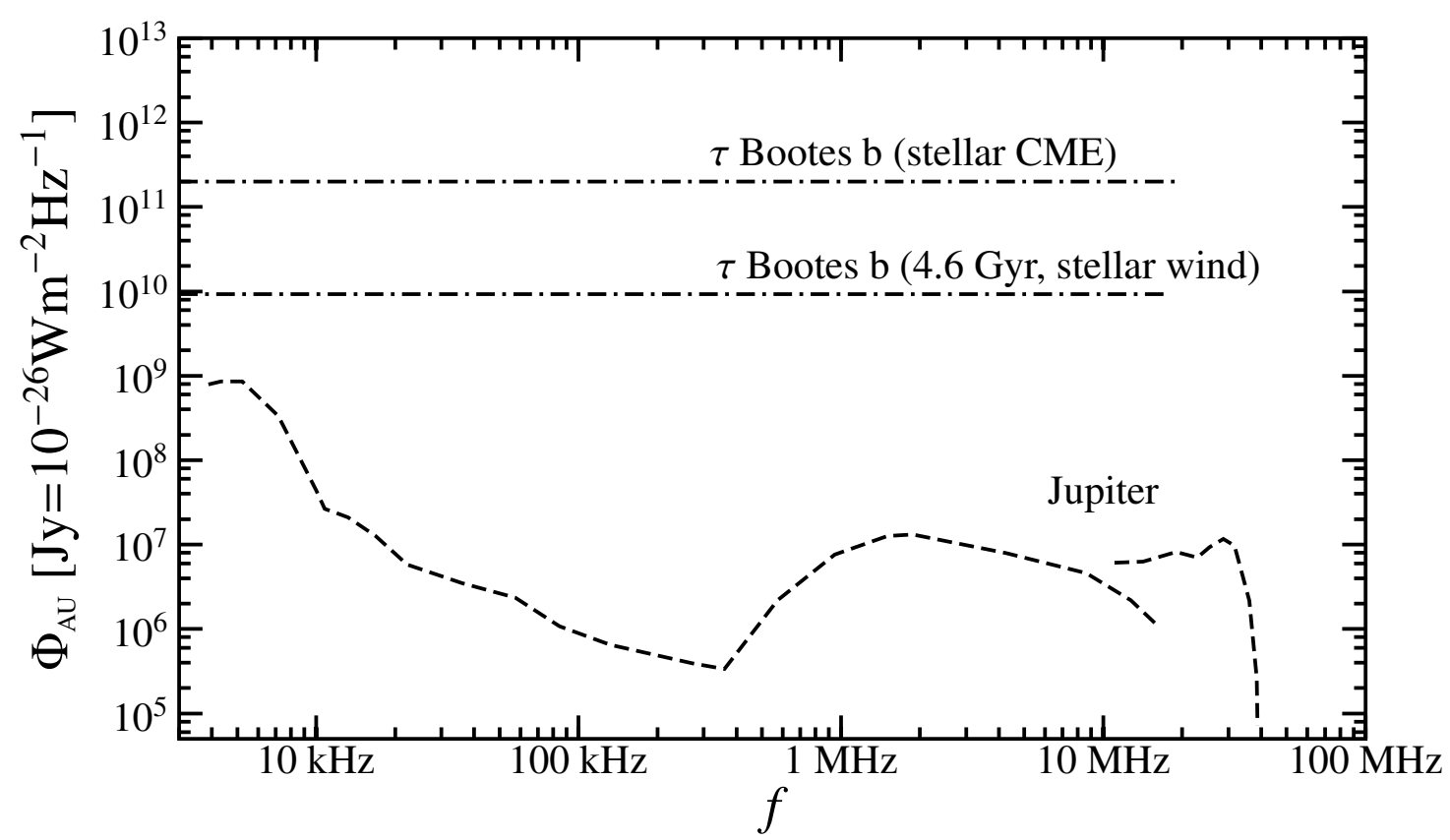

Figure 6.3: Radio flux expected for a planet similar to $\tau$ Bootes $\mathrm{b}$ around a $4.6 \mathrm{Gyr}$ old star. Both the radio flux energised by the steady stellar wind and that triggered by CME-like stellar coronal mass ejections are compared to the radio flux observed from Jupiter as given by Zarka et al. (1995, 2004). All values are taken at a distance of 1 AU.

driven by the stellar wind of a star of $4.6 \mathrm{Gyr}$ age, and b) radio emission driven by strong CMEs. One can clearly see that for Hot Jupiters around a star of $4.6 \mathrm{Gyr}$ age ${ }^{3}$, radio emission driven by a CME is much stronger than solar wind driven emission. In the case presented here, both types of emission differ by a factor of 20. The CME-driven radio flux is still considerably higher than the stellar wind-driven flux when $P_{\mathrm{rad}, \mathrm{J}}$ is set equal to $1.1 \cdot 10^{12} \mathrm{~W}$ instead of $2.1 \cdot 10^{11} \mathrm{~W}$ (i.e. using the peak flux instead of the average value for periods of high activity, see Table 6.1). This comparison shows that CMEs have to be taken into account when discussing exoplanetary radio emission.

Together with Section 6.1.3, these results show that when expected radio fluxes are calculated for different planets, not only the planetary parameters have to be considered. Stellar parameters like the age of the stellar system (Section 6.1.3) or the measured stellar coronal activity (Stevens 2005) can be used to deduce the stellar mass loss rate, which in turn has a strong influence on the expected radio flux. In addition to these parameters, the CME activity of the star is also an important parameter and should be considered when establishing target lists for observations of extrasolar planets in the radio frequency domain.

\footnotetext{
${ }^{3}$ This does not correspond to the age of $\tau$ Bootes, see Section 6.1.3 Thus, this section compares different radio fluxes of a 4.6 Gyr old $\tau$ Bootes-b-like planet.
} 


\subsection{Solar and stellar radio emission}

Besides the intensity of the planetary radio emission discussed in the previous section, the intensity of the emission by the planetary host star is an equally important criterion for the observation of planets by means of their radio emission. Similarly to measurements in the optical range, an observer will always see the combined signal of the star and its planet(s). This is true for observations of close-in planets $4^{4}$ in all spectral ranges, but the intensity ratio of stellar to planetary emission varies. From the calculation of theoretical spectra for wide-separation $(>0.2 \mathrm{AU})$ extrasolar giant planets, Burrows et al. (2004b) deduce a flux ratio of $\geq 10^{8}$ in the visible range and $\geq 10^{4}$ for infrared emission. For smaller separations, they find a flux ratio of $10^{3}$ in the mid-infrared. This is confirmed by infrared observations using the Spitzer Space Telescope, which found a drop of $7 \cdot 10^{-4}$ to $3 \cdot 10^{-3}$ in the measured intensity during secondary transits of HD $209458 \mathrm{~b}$ and TrES-1b (Deming et al.2005, Charbonneau et al. 2005). The situation is different for the low-frequency radio range, because planetary radio emission is dominated by powerful nonthermal emission generated by the cyclotron-maser-instability (CMI). The solar radio emission - which will serve as the main example for stellar radio emission in this section - consists of a quiet background (produced by thermal bremsstrahlung) plus a rich spectrum of radio bursts (caused by nonthermal electrons). The difference of the generation mechanisms is responsible for a much more favourable intensity ratio in the radio frequency range, thus making it easier to separate the stellar and the planetary radio emission, as will be shown below.

The following sections closely follow the discussion of Grießmeier et al. (2005a), in which the different contributions were systematically compared for the first time. The different types of solar radio emission are discussed in Section 6.2.1. In Section 6.2.2 a type of stellar radio emission is presented which does not occur on the Sun. These contributions to the stellar radio flux are compared to the planetary radio flux density in Section 6.2.3.

\subsubsection{Solar radio emission}

The solar radio flux is composed of different components, not all of which are always present. The components differ in intensity and rate of occurrence, and are caused by different emission mechanisms. According to Warmuth and Mann (2005), three different emission mechanisms are important:

- thermal bremsstrahlung generated by free electrons accelerated by the Coulomb fields of ions;

- gyro emission caused by electrons spiralling around magnetic field lines;

- coherent plasma emission close to the local electron plasma frequency or its harmonic excited by fast electrons.

In the following, the different components contributing to the solar radio flux are discussed in terms of their emission mechanism, commonness, peak intensity and relevance

\footnotetext{
${ }^{4}$ For planets with a large separation direct observation has recently become possible in the infrared, see Section 2.4
} 


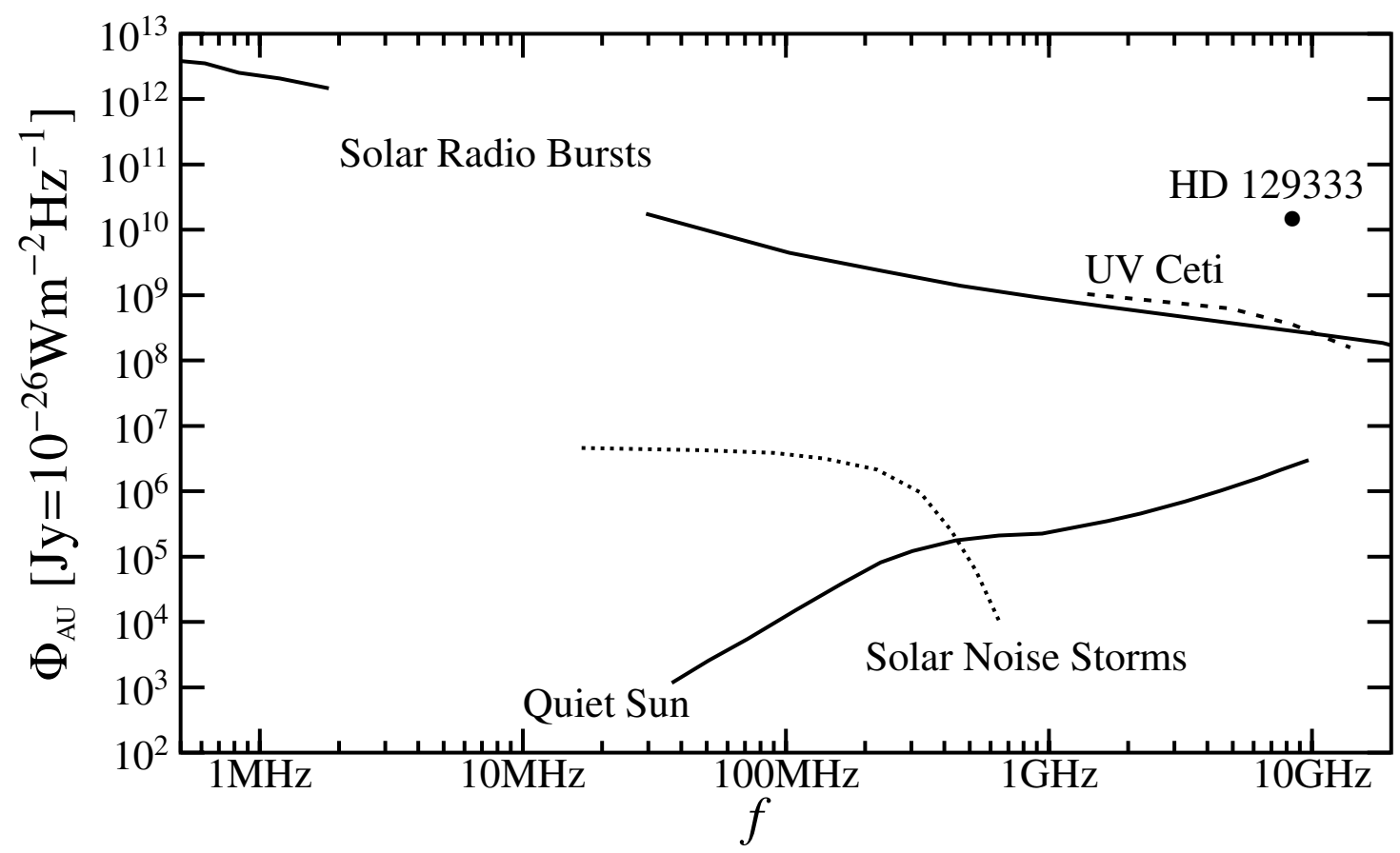

Figure 6.4: Components of the solar radio spectrum according to Boischot and Denisse (1964) (dotted line) and Nelson et al. (1985) (solid lines). The quiescent stellar emission of the dG0e star HD 129333 (EK Draconis) measured at $8.4 \mathrm{GHz}$ (Güdel et al. [1995) as well as that of the dM5.5e star UV Ceti (short dashed line, from Güdel and Benz 1996) are described in Section 6.2.2. All values are taken at a distance of $1 \mathrm{AU}$.

concerning the radio detection of extrasolar planets. Fig. 6.4 shows the intensity of the quiet sun and the observed maximum intensity of solar radio bursts as well as that of solar noise storms.

The quiet sun emission is caused by thermal bremsstrahlung due to electron-ion collisions in the ionised plasma. Observations at different frequencies typically yield information about different layers in the star. The higher the frequency $f$, the denser (and lower) the generating layer may have been. Most of the observed quiet sun radio emission originates from the solar atmosphere (and not from deep inside the Sun).

The slowly varying component (not shown in Fig. 6.4p is mainly due to gyrosynchrotron emission from regions of hot and dense plasma in the corona, e.g. over sunspots (Warmuth and Mann 2005). This leads to a flux density variation by a factor of about 2 at centimetre and decimetre wavelengths (Sheridan and McLean 1985). It has a periodicity of 27 days due to the solar rotation (Boischot and Denisse 1964) and varies with the sunspot cycle. While the quiet sun emission is randomly polarised (Sheridan and McLean 1985), the emission in the centimetric range is often circularly polarised, which can only be explained by the high magnetic fields of the sunspots (Boischot and Denisse 1964).

Solar radio bursts are generated by high-frequency plasma oscillations excited by suprathermal electrons. These plasma oscillations have to be converted into electromagnetic radiation. Solar radio bursts typically have much higher flux densities when compared to the quiet sun emission. They are observed in the whole frequency range, but they are more intense in the low frequency domain (see Fig. 6.4). The emission takes place 
close to the electron plasma frequency or its harmonics (Melrose 1985). Solar radio bursts are usually partially circularly polarised. Some types of solar radio bursts are briefly presented in the following; a more complete review is given by Warmuth and Mann (2005). Type I bursts only occur in large groups. These so-called "Noise Storms" are described below. Type II bursts are generated by magnetohydrodynamic shock waves caused by a disturbance moving with super-Alfvénic velocity. Suprathermal electrons in the shockfront region excite Langmuir waves which are converted to electromagnetic radiation. Type II bursts display a detailed fine structure (see e.g. Mel'nik et al. 2004). The polarisation is similar to that of type III bursts (see below). Type III bursts, characteristic for the impulsive phase of solar flares, are the most common flare-associated bursts. They are generated by relativistic electrons (typically $v \approx 0.3 c$, see Warmuth and Mann 2005). Because of the high particle velocity, a large frequency drift $d f / d t$ is characteristic for type III-bursts. For this type of emission, radio waves are emitted not only at the fundamental frequency of plasma waves, but also at their second harmonic (Bougeret et al. 1984). The polarisation degree ranges from weak $(<0.15)$ to moderately high $(\sim 0.5)$. Non-flare related type III bursts are found in type III storms (see below). The broadband emission of a type $I V$ burst is caused by energetic electrons trapped in a closed magnetic structure. Some of these structures are stationary, others move slowly upward, leading to a slow frequency drift. Type $V$ bursts are continuum emissions over a wide frequency range. They follow type III bursts and typically have the opposite circular polarisation with respect to the preceding type III burst.

Solar noise storms are frequently the dominating component of solar radio emission for wavelengths between 1 and $10 \mathrm{~m}$. Both flare and non-flare related noise storms exist. Two types of storms are distinguished, which are named type I storms and type III storms according to the type of associated radio bursts. Although the radio flux density associated with a type I noise storm is far below that of a radio burst, it can be 1000 times that of the quiet sun. Due to their occurrence rate and their duration, noise storms significantly contribute to the signal detected: Near solar maximum, noise storms occur about $10 \%$ of the time (Hjellming 1988). The typical duration of a noise storm is between a few hours and several days (Boischot and Denisse 1964, Warmuth and Mann 2005). Type I noise storms consist of a slowly varying broadband continuum plus short-lived bursts. The emission of type I storms is highly circularly polarised (Boischot and Denisse 1964 , Kai et al. 1985). Type III storms are not associated with flares; they are connected to type III bursts (see above). Type III storms can also include continuum emission in addition to bursts, but these continua only have a low intensity (Kai et al. 1985). Often, there is a temporal relationship between type I storms and type III storms, which is possibly due to a common exciting agent (Kai et al. 1985). Type III storms always have the same polarisation as type I storms, but their degree of polarisation is usually much lower.

\subsubsection{Stellar radio emission}

Some stars are continuously emitting much more energy at radio frequencies than the Sun. These radio luminosities can be 2-3 orders of magnitudes higher than the quiet sun (Benz 1993). This kind of emission is probably due to nonthermal processes (possibly gyrosynchrotron emission of energetic electrons); to emphasise the different generation mechanism of this emission with respect to the quiet sun emission, the term quiescent 
radio emission was introduced. There is no corresponding type of radiation on the Sun. The typical variation of the quiescent emission is about $50 \%$ on a time scale of hours, and it has a low degree of polarisation (Benz 1993). Unfortunately, measurements of stellar radio spectra are limited to only few frequencies. Also, no data are available for frequencies below $1 \mathrm{GHz}$. Fig. 6.4 shows a spectrum for the quiescent stellar emission of the dM5.5e star UV Ceti (Güdel and Benz 1996, dataset 4), calculated for a distance of 1 AU. The stellar distance was taken to be 2.627 pc (calculated from Gliese and Jahreiß 1991). Güdel and Zucker (2000) fitted four-point radio spectra observed by the VLA to the gyrosynchrotron model of White et al. (1989). This fit indicates that for UV Ceti, the maximum in the emission spectrum probably lies above $1 \mathrm{GHz}$, so that lower flux densities can be expected for lower frequencies. For frequencies below this maximum, optically thick emission with an intensity proportional to $f^{2.5}$ is expected. In reality, exponents between 0 and 10 can be found (Benz 1993).

Moreover, stars with even higher quiescent radio flux exist. The quiescent radio flux decreases with increasing stellar age (Güdel et al. 1998). For this reason, the emission of a young star can serve as an upper limit for quiescent emission. Fig. 6.4 shows the quiescent stellar emission of the young (approx. $70 \mathrm{Myr}$, see Dorren and Guinan 1994) dG0e star HD 129333 (EK Draconis) measured at the frequency of $8.4 \mathrm{GHz}$ (Güdel et al. 1995). The emission of HD 129333 will be used as the upper limit for the contribution of the quiescent emission.

It is known that stellar flares can be much more energetic than solar flares; stellar flares with $10^{4}$ times the radio flux of the largest solar radio burst have been observed. These flares are often completely circularly polarised (Güdel et al. 1989, Benz 1993). The influence of both stellar flares and quiescent radio emission on the detectability of planets is discussed in section 6.2.3.

Some very large flares (up to $10^{7}$ times more energetic than the largest solar flare) on solar-like stars could possibly be caused by the interaction of a normal F or G dwarf star and a magnetised close-in extrasolar planet (Rubenstein and Schaefer 2000). However, so far only nine of these transient extreme events were detected (Schaefer et al. 2000). For this reason, these so-called "super-flares" do not present a systematic problem for the discrimination of stellar and planetary radio emission. Note also that none of these stars have known close-in exoplanets.

\subsubsection{Comparison of solar, stellar and exoplanetary radio fluxes}

In this section, the flux densities of different radio sources are compared. Fig. 6.5 shows the flux densities of all contributions discussed in the previous section. To facilitate the comparison, all flux densities are given for a distance of 1 AU.

First, the contribution of the galactic background is considered. It is known that the galactic background depends on the viewing direction. It can be measured by a second measurement of the sky close to the extrasolar system and then be subtracted from the signal received from the system. For example, the UTR-2 radio telescope in Kharkov can be used in a two-beam mode with one of the beams directly on the radio source and the second beam pointing $1^{\circ}$ away from the first beam (Zarka et al. 1997). Another option would be to remove the sky background by means of interferometry, see e.g. Nelson et al. (1985). 
Comparing Jupiter's radio emission to the Sun's emission, one can clearly see that the planetary emission is far more powerful than the quiet sun emission. Thus, during quiet conditions, it would be difficult to detect radio emission emanating from a Sun-like star, but planetary radio emission would be detectable without confusion by stellar emission. The slowly varying component (not shown in Fig. 6.5) does not contribute much to the solar flux in the spectral range where planetary emission is expected.

The quiescent emission exhibited by some stars could in principle be problematic. Although no measurements are available for low frequencies $(\lesssim 1 \mathrm{GHz})$, it seems likely that the flux levels are much lower at frequencies relevant for planetary detection $(\lesssim 25$ $\mathrm{MHz}$, see Table 6.2). Also, the quiescent radio emission seems to be connected to stellar $\mathrm{X}$-ray emission (Güdel et al. 1995). Thus, the comparison of the radio emission and the $\mathrm{X}$-ray emission might serve as an indicator whether the source of the radio emission is likely to be the star or not. Furthermore, the low degree of polarisation of the quiescent emission (see Section 6.2.2) as opposed to the planetary radio emission will prove to be an important diagnostic tool.

Due to their relatively low occurrence-rate (about $10 \%$ of the time near solar maximum, see section 6.2.1, noise storms are not very important for the case where a Jupiterlike planet is to be detected around a Sun-like star. They may have an influence for systems where either the planetary radio emission is weaker than Jupiter's, or in cases of a star showing more activity than the Sun. In these cases, statistical considerations would be required, and the quiet star level would have to be evaluated carefully. In Section 6.1.2. it was shown that for close-in extrasolar planets much stronger emission is expected than for Jupiter. Thus the contribution of noise storms is probably negligible.

Stellar radio bursts are another matter. In the solar system, they are far more powerful than any planetary radio emission. The question arises as to whether the latter could be separated from such a bursty background. Fortunately, these radio bursts do not occur all the time. Although type IV emission can last for several days, they only occur with a rate of approximately 3 per month during sunspot maximum. Type III emission take place much more frequently (up to 1400 per month at sunspot maximum), but their duration is limited to a few seconds (Boischot and Denisse 1964). Thus it can be hoped that using statistical arguments the stellar bursts can be separated from planetary emission. If one admits the possibility that the beaming direction of extrasolar planetary radio emission could be very different from that observed in the solar system, it could be worthwhile to look at secondary eclipses of transiting planets. This technique is successfully employed in the infrared (see Section 2.4). Similarly, for radio frequencies, different spectra may be obtained during secondary transit ("star only"-spectra when the planet passes behind the star) and off-eclipse ("star plus planet"-spectra). The noisy background of the stellar radio bursts could then be reduced by statistics, i.e. by observing not one but many secondary transits of an appropriate planet. Of course, a star with little activity in the radio spectrum (like the Sun at solar minimum) is preferable.

The planetary radio emission of some extrasolar planets may be much stronger than Jupiter's emission. For example, for a system similar to $\tau$ Bootes, the radio emission will be several orders of magnitude stronger than Jupiter's emission (see Fig. 6.2). It can be seen that detection is more likely for young stellar systems, where the stellar wind is denser and faster than for the present day Sun. The radio emission from $\tau$ Bootes $\mathbf{b}$ at its present age $(1.0 \mathrm{Gyr})$ is much stronger than the contributions of the galactic background, 


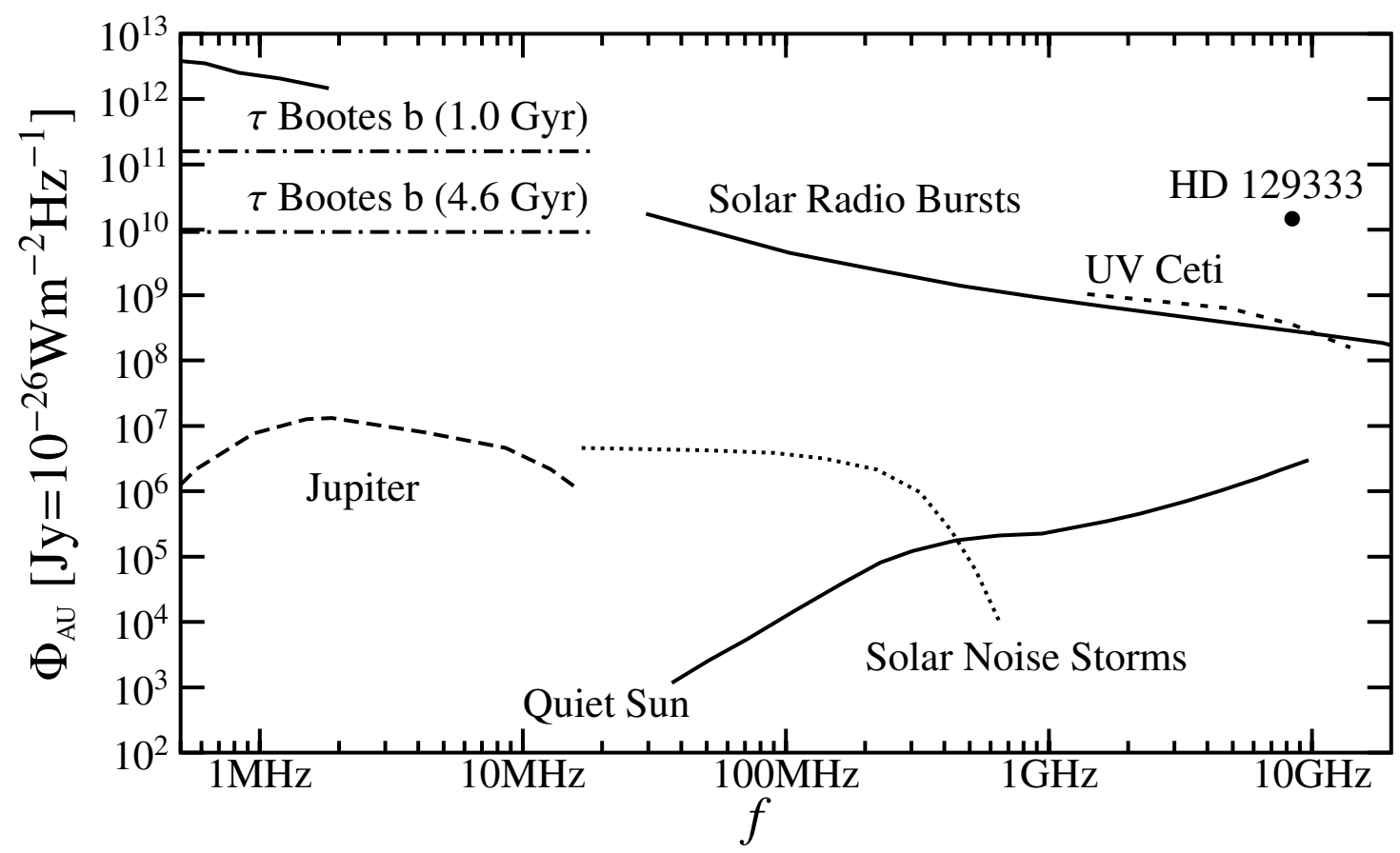

Figure 6.5: Solar radio data according to Boischot and Denisse (1964) (dotted line) and Nelson et al. (1985) (solid lines). Jupiter radio flux during periods of intense activity (cf. Fig. 6.1) according to Zarka et al. (2004) (dashed line). Also shown are the quiescent stellar emission from UV Ceti (short-dashed line) and HD 129333 (see Section 6.2.2) as well as the lower limit for the radio flux expected from $\tau$ Bootes (dash-dotted lines, see Section 6.1.3). All values are given for a distance of $1 \mathrm{AU}$.

the quiet sun emission, solar noise storms, and also the assumed maximum quiescent radio emission. Some stellar radio bursts will still be more intense than the planetary emission; depending on the occurrence rate of radio bursts on the star, this might be more or less problematic. This is especially true if the planets host star exhibits very strong stellar flares.

Even in cases where the combined stellar/planetary radio signal contains major contributions from the planet, one also requires some means to separate the two contributions. There are several ways how this can be achieved:

- It is known that planetary radio emission is highly polarised (Zarka 1998). This is not the case for the quiet sun radio emission (see Section 6.2.1) or the quiescent radio emission (see Section 6.2.2).

- The bursty component could possibly be discriminated by occultation during secondary eclipses.

- It is known from the solar system that planetary radio emission is modulated with the planetary rotation period, which is considerably shorter than the stellar rotation period. For this reason, rotational modulation of the observed radio emission could help to distinguish the stellar and the planetary contribution. Of course, this would work best for planets not subjected to tidal locking (i.e. far enough from their star, 
see Section 3.1). For tidally locked Hot Jupiters, the planetary rotation period is of the order of a few days, so that this method may still be applicable. It will fail in the few cases where the stellar and the planetary rotation are synchronised. As discussed by Pätzold and Rauer (2002), some measurements of the stellar rotation period of $\tau$ Bootes seem to indicate that this is the case (this is also suggested by Leigh et al. 2003), while other values suggest that the star is rotating more slowly. Even if the method is not applicable to $\tau$ Bootes, the modulation with the rotation period may be very useful for other stellar systems.

\subsection{Observation of exoplanetary radio emission}

Over the last decades, several attempts were undertaken to detect radio emission from extrasolar planets. So far, however, these efforts have been unsuccessful. The different attempts to observe exoplanetary radio emission are presented in Section 6.3.1. In Section 6.3.2, the observed frequencies and achieved radio flux sensitivities of these observations are compared to the predicted radio fluxes from Section 6.1. It will be shown that the reason for the current non-detection is the lack of sensitivity in the appropriate frequency range. At the same time, the prospects for future observations are analysed. It will be shown that the radio flux anticipated for certain planets is strong enough to allow groundbased detection in the near future.

\subsubsection{Observational attempts}

The first attempts to discover the radio emission of extrasolar planets date back to long before the announcement of the first extrasolar planet. In recent years, when targeted radio observations became possible due to the knowledge of the position of extrasolar planets, new efforts were undertaken to search for such radio emission. So far, however, all such efforts have been unsuccessful. This includes:

- Early observations by Yantis et al. (1977), where 22 nearby stars (i.e. within 5 parsec) were observed using the Clark Lake radio telescope at a frequency of 26.3 $\mathrm{MHz}$.

- The observation of 6 nearby stars (i.e. within 5 parsec) by Winglee et al. (1986) using the Very Large Array (VLA). Frequencies of $330 \mathrm{MHz}$ and $1460 \mathrm{MHz}$ were used, and the typical observation time was $3.5 \mathrm{~h}$. One star was additionally observed for 1 hour at $4.9 \mathrm{GHz}$.

- Observations made between 1994 and 1996 by the Ukrainian radio array UTR-2 in the decametric wavelength range (Zarka et al. 1997). 4 stars were observed for approximately 45 minutes each.

- VLA observations of 10 stars made between 1996 and 1998. The planet-hosting target stars had distances between 2.5 and 41 pc (Bastian et al. 2000). Observations were performed at $74 \mathrm{MHz}, 333 \mathrm{MHz}$ and $1465 \mathrm{MHz}$, with an observation time of $\lesssim 6$ hours per star. 
- Targeted observations of $\tau$ Bootes (1999 and 2002), using the VLA (Farrell et al. 2003). The time per measurement was $\lesssim 6$ hours.

- The analysis of the VLA Low-frequency Sky Survey (VLSS), an ongoing effort to survey the northern sky at $74 \mathrm{MHz}$ (Lazio et al. 2004). While not as sensitive as targeted observations, a much larger number of stars will be observed eventually.

- A search for decametric radio bursts using the UTR-2 radio array (Ryabov et al. 2004). Between 1999 and 2002, 19 stars were observed, with an observation time of typically several hours per star. For $\tau$ Bootes, the sensitivity reached $160 \mathrm{mJy}$.

- An observation of HD 209458 using the Effelsberg $100 \mathrm{~m}$ radio telescope. On March 31, 2004, the system was observed for 1 hour out of transit (E. Guenther, personal communication, 2005).

- Observations made with the $10 \mathrm{~m}$ Mizusawa telescope and $11 \mathrm{~m}$ Tokai university telescope since 1996 (Y. Shiratori, personal communication, 2005). 4 stars were observed at a frequency of $8.6 \mathrm{GHz}$ with a sensitivity of approximately $1 \mathrm{Jy}$. More than 100 hours of observational data were collected per target.

- The observation of two targets with the Giant Metrewave Radiotelescope (GMRT) in India (Majid et al.2006). The data of these observations are still under examination.

\begin{tabular}{|c|c|c|c|}
\hline Telescope & Frequency & Sensitivity & Reference \\
\hline Clark Lake & $26.3 \mathrm{MHz}$ & $1 \mathrm{Jy}$ & Yantis et al. (1977) \\
\hline VLA & $330 \mathrm{MHz}$ & $30 \mathrm{mJy}$ & Winglee et al. (1986) \\
\hline & $1460 \mathrm{MHz}$ & $0.3 \mathrm{mJy}$ & \\
\hline & $4900 \mathrm{MHz}$ & $0.15 \mathrm{mJy}$ & \\
\hline UTR-2 & 7-35 MHz & 2-4 Jy & Zarka et al. (1997) \\
\hline VLA & $74 \mathrm{MHz}$ & $50 \mathrm{mJy}$ & Bastian et al. (2000) \\
\hline & $333 \mathrm{MHz}$ & $1-10 \mathrm{mJy}$ & \\
\hline & $1465 \mathrm{MHz}$ & $0.02-0.07 \mathrm{mJy}$ & \\
\hline VLA & $74 \mathrm{MHz}$ & $120 \mathrm{mJy}$ & Farrell et al. (2003) \\
\hline VLA & $74 \mathrm{MHz}$ & $300 \mathrm{mJy}$ & Lazio et al. (2004) \\
\hline UTR-2 & $18-32 \mathrm{MHz}$ & 100-1600 mJy & Ryabov et al. (2004) \\
\hline Effelsberg & $4850 \mathrm{MHz}$ & $0.8 \mathrm{mJy}$ & E. Guenther, pers. communication, 2005 \\
\hline Mizusawa & $8600 \mathrm{MHz}$ & $1 \mathrm{Jy}$ & Y. Shiratori, pers. communication, 2005 \\
\hline GMRT & $153 \mathrm{MHz}$ & $2 \mathrm{mJy}$ & Majid et al. (2006) \\
\hline
\end{tabular}

Table 6.5: Past attempts to detect radio emission from extrasolar planets (taken from Grießmeier et al. 2006a).

Many reasons were suggested to explain why these searches for radio emission from Hot Jupiters have not yet proven successful (see, e.g. Bastian et al. 2000, Ryabov et al. 
2004). The most fundamental problem of these observations probably is the lack of sensitivity in the appropriate frequency range. This becomes clear when the frequencies and sensitivities of the observation attempts (compiled in Table 6.5) are compared to the values expected for exoplanetary radio emission, which is done in the next section.

\subsubsection{Estimated radio flux}

If one compares the different Hot Jupiters of Tables 6.2 and 6.3 with the frequency constraints imposed by the Earth's ionospheric cutoff ( . . 10 MHz, see Zarka et al. 1997) and with the observation campaigns of Table 6.5, one finds the following:

- Due to the Earth's ionosphere, radio emission from the planets HD 209458b and OGLE-TR-10b is not accessible for ground-based observations. The reason for this is the weak planetary magnetic field, which is caused by tidal locking (Section 3.1).

- The maximum emission frequency for the planets OGLE-TR-111b and TrES-1b is close to the ionospheric cutoff frequency, rendering observations very difficult. None of the past observation attempts included frequencies low enough to include the emission frequencies of these planets.

- For OGLE-TR-56b, OGLE-TR-113b, and OGLE-TR-132b, the ionospheric cutoff is not a problem, and observations at low enough frequencies were performed. The planets are expected to be powerful radio emitters. This is especially true for OGLE-TR-56b, which has the largest emitted radio power $P_{\text {rad }}$. However, because of the extremely large distance of these planets, the flux density at Earth is much too low to allow detection. Although these planets are no good candidates for radio observations, they can serve as representatives of a certain class of planets. Other planets very similar to these could exist at much closer distances to the stellar system. For such planets, radio detection would be possible.

- The planet around the star $\tau$ Bootes clearly is the most promising candidate of all planets included in this study. It has both the highest emission frequencies and the largest expected radio flux. The radio flux $\Phi_{s}$ of $\tau$ Bootes is compared to the sensitivities reached by different observation campaigns in Figure 6.6. Radio flux densities up to $40 \mathrm{mJy}$ are expected. In the worst case (i.e. the "light" model), the flux density is lower by a factor of three (see Table 6.3). Concerning the current non-detection of radio emission from $\tau$ Bootes $b$, the main problem is the relatively low maximum frequency of $19 \mathrm{MHz}$ of the emission as compared to the majority of measurements which took place at frequencies $f \geq 74 \mathrm{MHz}$ (see Table 6.5 and Figure 6.6). The observations of Zarka et al. (1997) and Ryabov et al. (2004) are in a more promising frequency range (between 7 and $35 \mathrm{MHz}$ ), but the sensitivity reached is not yet sufficient. The observations by Ryabov et al. (2004) come close to the parameter range where the detection of radio emission from $\tau$ Bootes is possible. For this reason, future UTR-2 observations will either lead to a positive detection or impose important constraints on the planetary magnetic moment.

The radio flux $\Phi_{s}$ from Table 6.3 can also be compared to the detection limit of the planned LOw Frequency ARray (LOFAR, described by Kassim et al. 2004), which will 


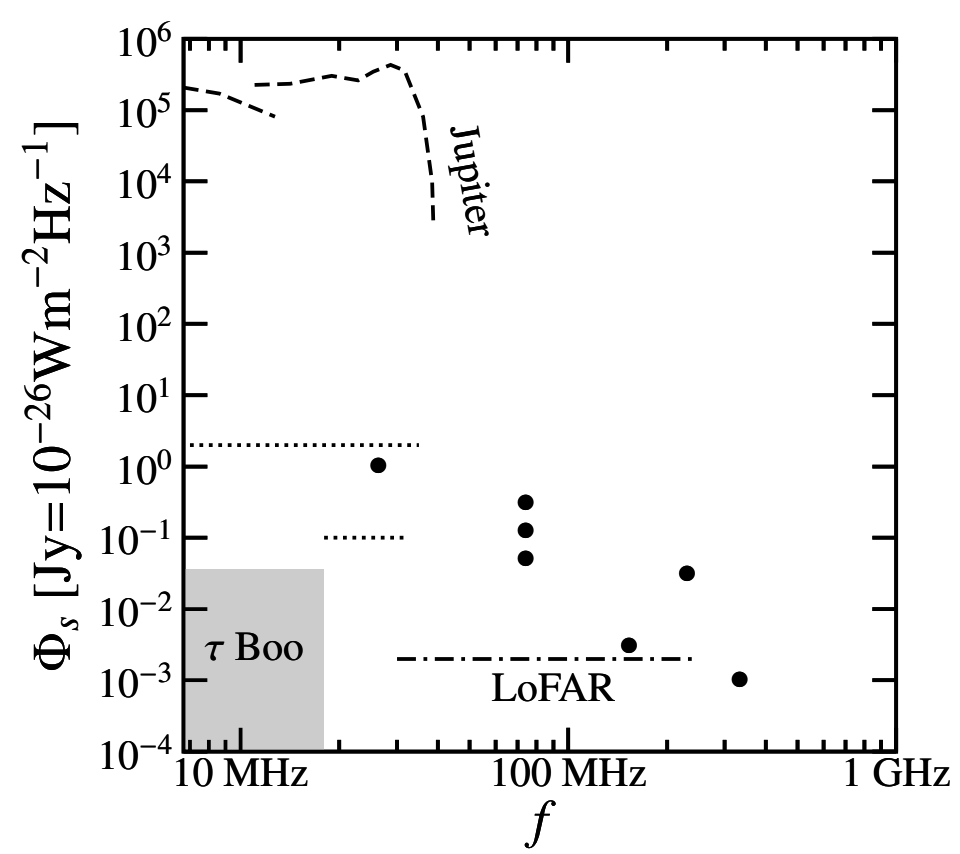

Figure 6.6: Comparison of the radio flux measured from Jupiter (cf. Fig. 6.1) according to Zarka et al. $(1995,2004)$ at periods of intense activity (dashed lines) and the lower limit for the radio flux emission from $\tau$ Bootes $\mathrm{b}$ (shaded area). Also shown: previous observations (dotted lines and points, see Table 6.5 and the planned LOFAR detector (dash-dotted line).

go into operation in 2007-2008. It will consist of an array of phased dipoles with separations of up to $400 \mathrm{~km}$ between different station, and is currently under construction in the Netherlands, which allows for an extremely low sensitivity. However, according to the modified instrument design plans, LOFAR will only include frequencies above $30 \mathrm{MHz}$ (instead of the previously planned $10 \mathrm{MHz}$ ) with a sensitivity of approximately $2 \mathrm{mJy}$ (Zaroubi and Silk 2005, and see also http: / / www . lofar.org). Because of the modified frequency range, the detection of planetary radio emission by this instrument is uncertain.

Ongoing efforts at the VLA and at the GMRT are limited to higher frequencies (i.e. to $74 \mathrm{MHz}$ and $153 \mathrm{MHz}$, respectively). At the GMRT, observations at $50 \mathrm{MHz}$ will be made possible by the installation of a new feed. While this frequency is still higher than those of Table 6.2, other planets may exist which emit at this frequency. At the UTR-2 radio array, the sensitivity of the observations will be improved using a new digital receiver in the near future. With the capability of that array to observe at low frequencies, it seems possible that this instrument will achieve the first detection of extrasolar planetary radio emission. 


\title{
7 Protection of terrestrial exoplanets against galactic cosmic rays
}

\author{
C'est une bien faible lumière qui nous vient du ciel étoilé. \\ Que serait pourtant la pensée humaine si nous ne pouvions \\ pas percevoir ces étoiles... \\ Jean Perrin, french physicist (1870-1942)
}

One of the most fascinating questions in astrobiology is the search for habitable worlds. Current studies on the surface habitability of extrasolar Earth-like planets mostly focus on the existence of liquid water on the planetary surface. While this is a necessary condition for life as we know it, this condition is far from being sufficient. Especially for weakly magnetised planets, additional aspects have to be studied, see Section 2.3. One of these aspects is the impact of galactic cosmic rays. Because of their relatively small mass, low luminosity and large abundance, $\mathrm{M}$ dwarfs are considered prime targets for searches for terrestrial habitable planets, like ESA's DARWIN or NASA's TPF-C mission. However, extrasolar planets in the habitable zone of $\mathrm{M}$ stars are in synchronous rotation around their host star because of tidal locking (Section 3.1.6). This leads to strongly reduced rotation rates, which in turn are responsible for relatively small magnetic moments (Section 4.6). For example, an Earth-like extrasolar planet which is tidally locked in an orbit of $0.2 \mathrm{AU}$ around a star of $0.5 M_{\odot}$ (i.e. within the liquid water habitable zone described in Section 2.3 has a magnetic moment of less than $15 \%$ of the Earth's current magnetic moment. Correspondingly, a much smaller magnetosphere is to be expected (Section 5.3.4). Therefore, such a close-in extrasolar planet is not well protected by an extended magnetosphere, and cosmic rays can reach almost the whole surface area of the upper atmosphere.

In this chapter, the resulting flux of galactic cosmic rays to extrasolar planets is discussed. A short introduction on cosmic rays is given in Section 7.1. Section 7.2 describes how the cosmic ray trajectories are calculated and defines the impact area and the energy spectrum of the particles, which are used to quantify the cosmic ray particle flux. The cosmic ray flux is analysed for different exoplanetary situations in Section 7.3. Potential biological effects and the influence on habitability are discussed in Section 7.4. This chapter is based on Grießmeier et al. (2005b) and Grießmeier et al. (2006d), but includes some more recent results. 


\subsection{Galactic cosmic rays}

Generally, the term "cosmic rays" denotes all energetic ionised particles originating from space. Usually, different classes of cosmic rays are distinguished:

- Galactic cosmic rays (GCR) are particles accelerated to high energies at distant astrophysical sources such as quasars, supernovae, neutron stars, star-forming regions, $\gamma$-ray bursts, and jets from black holes. At Earth, they present an approximately homogeneous background and reach the Earth with the same intensity from all directions (Meyer et al. 1974). Of all different types of cosmic rays, they have the highest energies. During the solar cycle the flux of GCR with energies around $100 \mathrm{MeV}$ varies by a factor of approximately two (Seo et al. 1994). While GCR comprise all nuclei of the periodic table of elements, protons are by far the most abundant species of GCR.

- Anomalous cosmic rays (ACR) are particles accelerated at the heliospheric shock. Close to the Sun, ACR do not contribute much to the total particle flux (Scherer et al.2002).

- Short phases with large fluxes of solar (or, by analogy, stellar) cosmic rays are associated to interplanetary shocks, e.g. those created by fast CMEs. However, not all interplanetary shocks lead to an enhancement of the solar cosmic ray flux (Smart) and Shea 2002). At low energies (i.e. below $130 \mathrm{MeV}$ ), the yearly average of the flux of solar protons is larger than that of GCR protons (Kuznetsov and Nymmik 2002).

- Planetary particles which originate from planetary magnetospheres (mostly from Jupiter's magnetosphere in the case of the solar system). Concerning the total particle flux, these particles only play a minor role.

General introductions to galactic cosmic rays are provided by Meyer et al. (1974), Kallenrode (2000), and McKibben (2001). The cosmic ray calculations of this chapter deal exclusively with GCR protons.

\subsection{Cosmic ray calculation}

In order to quantify the shielding effect of the planetary magnetosphere, the motion of galactic cosmic protons in the model magnetosphere described in Section 5.3.1 is investigated. The calculations were performed with the software package EPOM (Stadelmann 2005b). In this section, these calculations are briefly described. It goes beyond the scope of this work to explain all the details of the numerical calculation. The interested reader is referred to the descriptions of Stadelmann (2005a).

\subsubsection{Calculation of particle trajectories}

In order to determine the impact of GCR protons in the energy range $64 \mathrm{MeV}<E<$ $8 \mathrm{GeV}$ on the planetary atmosphere, particle trajectories in the magnetosphere are anal- 


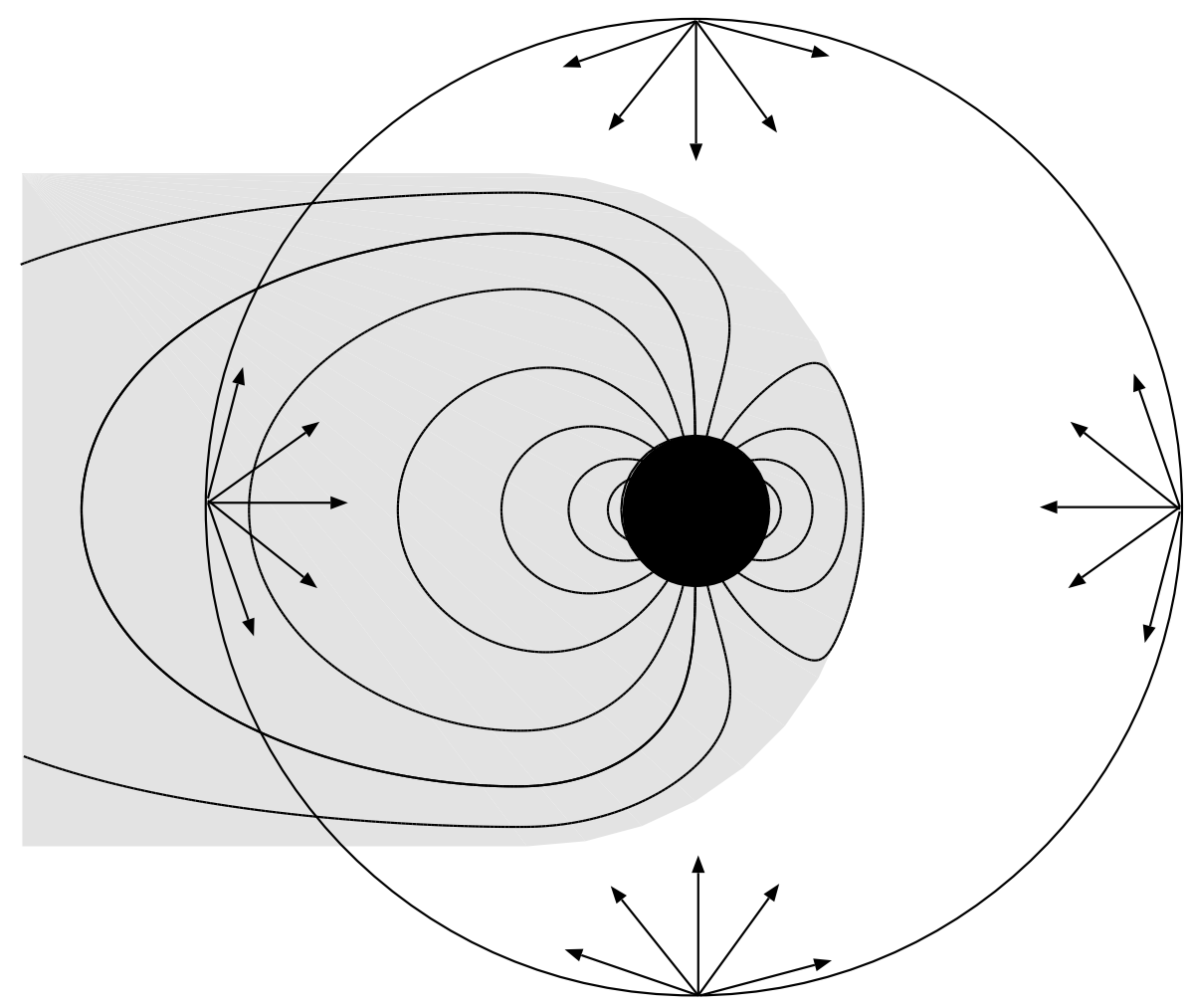

Figure 7.1: Distribution of the starting positions and velocity vectors for cosmic ray trajectories (schematic view).

ysed. Because no solution in closed form exists, this is only possible through the numerical integration of many individual trajectories (Smart et al. 2000). For each particle energy (64 MeV, $128 \mathrm{MeV}, 256 \mathrm{MeV}, 512 \mathrm{MeV}, 1024 \mathrm{MeV}, 2048 \mathrm{MeV}, 4096 \mathrm{MeV}$ and $8192 \mathrm{MeV}$ ) and for each magnetospheric configuration, over 7 million trajectories are calculated, which correspond to protons with different starting positions and starting velocity directions. The particles are launched from the surface of a sphere centred on the planet. The radius of this sphere satisfies the condition $r \geq R_{\mathrm{s}}$, so that the particles (except for those arriving from the tailward direction) are launched outside the magnetosphere, see Fig. 7.1.

Once the particles enter the magnetosphere, their motion is influenced by the magnetospheric magnetic field. This magnetic field is calculated with the closed magnetospheric model from Section 5.3.1, using the magnetic moment from Section 4.6 and the size of the magnetosphere from Section 5.3.4 as input. Note that the maximum magnetic moments from Table 4.7 are used. For this reason, the results represent the lower limit for the cosmic ray flux to the atmosphere. For a smaller magnetic moment, a larger cosmic ray flux is possible. The trajectories are calculated using the numerical Leapfrog method (Stadelmann 2005a). For each energy, all particles are counted which reach the atmosphere described by a spherical shell one hundred kilometres above the planetary surface, i.e. $R_{\mathrm{a}}=R_{\mathrm{p}}+100 \mathrm{~km}$. The impact of particles on the planetary atmosphere can be quantified by the impact area and the energy spectrum. These quantities are defined in the following section. 


\subsubsection{Cosmic ray impact area}

Because the strength and direction of the planetary magnetic field varies with latitude, the number of cosmic ray particles reaching the planetary atmosphere varies, too. The longitude, however, does not influence the influx of cosmic ray particles. In Figure 7.2, cosmic ray penetration through the magnetosphere is shown for the case of an Earth-like planet which is tidally locked at $0.2 \mathrm{AU}$ around a star with $M_{\star}=0.5 M_{\odot}$. This corresponds to the magnetic field configuration of Fig. 5.5. Obviously, the cosmic ray flux depends on the energy of the particles. High energy particles can penetrate the magnetosphere at any location, see Figure 7.2(c) and (d), while for lower energies, shown in Figure 7.2(a) and 7.2(b), less particles (or even no particles at all) reach the equatorial region. In the vicinity of the poles the magnetic field is almost radial, and particles of any energy can reach the top of the planetary atmosphere.

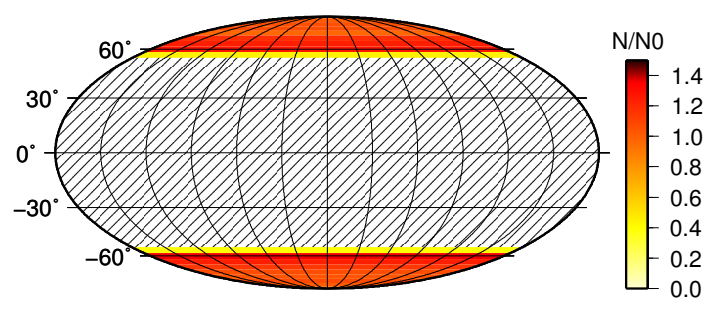

(a) Impact region for particles of $64 \mathrm{MeV}$.

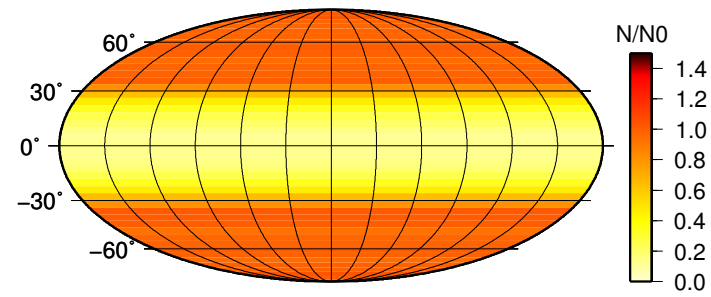

(c) Impact region for particles of $1024 \mathrm{MeV}$.

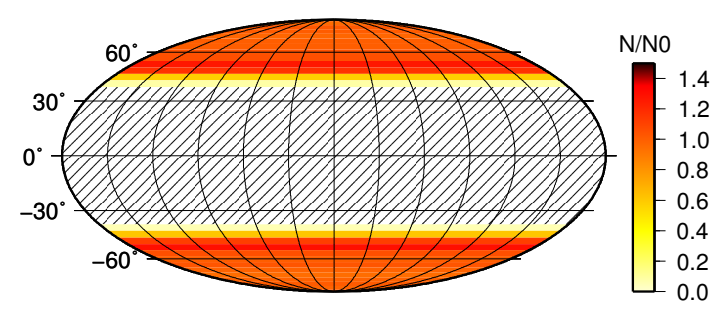

(b) Impact region for particles of $256 \mathrm{MeV}$.

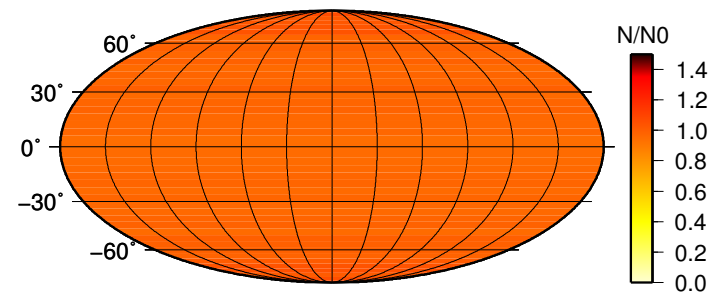

(d) Impact region for particles of $4096 \mathrm{MeV}$.

Figure 7.2: Particle impact for cosmic ray protons with an energy of $64 \mathrm{MeV}, 256 \mathrm{MeV}, 1024$ $\mathrm{MeV}$ and $4096 \mathrm{MeV}$ for an Earth-like planet which is tidally locked at $0.2 \mathrm{AU}$ around a star of $0.5 M_{\odot}$ (i.e. "locked case", see below). Colour-coded: Particle flux relative to a planet without magnetic field. In the hatched area, no particles reach the atmosphere.

For a given energy, the impact area is defined as the area where cosmic ray particles reach the planetary atmosphere, normalised to the planetary surface area. In Figure 7.2. for example, the impact area is equal to unity for energies above $1024 \mathrm{MeV}$. Protons with an energy of $256 \mathrm{MeV}$ can reach about a quarter of the atmospheric surface.

\subsubsection{Cosmic ray energy spectrum}

The cosmic ray energy spectrum is determined in the following way: for a given particle energy, the fraction of particles reaching the planetary atmosphere is registered. This 
value is compared to the fraction of particles reaching the atmosphere of an identical, but unmagnetised planet. The resulting magnetospheric filter function is multiplied with the cosmic ray energy spectrum outside the magnetosphere. This reference spectrum was taken from Seo et al. (1994).

\subsection{Cosmic rays in exomagnetospheres}

In the following, the flux of cosmic rays will be compared for different planets. The impact area and energy spectrum are determined for the following cases:

- "Earth case": Magnetosphere of the Earth. Here, the Earth's magnetic field is represented by a zonal dipole with a magnetic dipole moment of $\mathcal{M}=8 \cdot 10^{22} \mathrm{Am}^{2}$.

- "Locked case": Magnetosphere of an extrasolar planet which is tidally locked in an orbit of $0.2 \mathrm{AU}$ around a $0.5 M_{\odot} \mathrm{K} / \mathrm{M}$ star of $4.6 \mathrm{Gyr}$ age.

- "Unlocked case": Similar to the "locked case", but without tidal locking. Instead, the planet is assumed to have the magnetic moment of the Earth. Note that this case is not realistic (unless the planet was brought to this position only recently), but it is used to demonstrate the influence of tidal locking.

- "Unmagnetised case": For the cosmic ray energy spectrum, the case of a totally unmagnetised planet is shown for comparison. This corresponds to the cosmic ray energy spectrum outside a planetary magnetosphere.

- "Young star case": Similar to the "locked case", but for stellar wind conditions found around a star of 0.7 Gyr age.

- "Large Earth case": Similar to the "locked case", but for a large terrestrial planet (see Section 2.6.3). For distances of $d \geq 0.197$ AU this type of planet should be considered as "potentially locked" rather than "tidally locked" (cf. Table 5.5). In this Chapter, however, a tidally locked planet at $0.2 \mathrm{AU}$ is assumed.

- "Ocean Planet case": Similar to the "locked case", but for an Ocean Planet (see Section 2.6.3.

For the above cases, the magnetic moments and standoff distances are given in Table 7.1. Most of these values already appeared in Table 5.5. As noted before, the magnetic moments (and thus also the standoff distances) are assumed to have the maximum allowed value. Thus the values obtained in this chapter represent a lower limit for the flux of cosmic ray protons to the planetary atmosphere. Table 7.1 also includes the magnetic field strength at the magnetopause, which was obtained from eq. (5.43) and (5.44). This value is determined by the stellar wind pressure.

\subsubsection{Impact of cosmic rays on Earth-like exoplanets}

For the "locked case", the magnetosphere is much smaller than for the "Earth case", see Table 7.1. For this reason, a much higher flux of cosmic ray protons through the planetary magnetosphere can be expected. 
7 Protection of terrestrial exoplanets against galactic cosmic rays

\begin{tabular}{|l||c|c|c|}
\hline Planet & $\mathcal{M}\left[\mathcal{M}_{\mathrm{E}}\right]$ & $R_{\mathrm{s}}\left[R_{\mathrm{p}}\right]$ & $B\left(R_{\mathrm{s}}\right)[\mathrm{nT}]$ \\
\hline \hline Earth case & 1.0 & 9.91 & 73 \\
\hline Locked case & 0.15 & 4.12 & 150 \\
\hline Unlocked case & 1.0 & 7.81 & 150 \\
\hline Unmagnetised case & 0 & - & - \\
\hline Young star case & 0.15 & 2.31 & 1280 \\
\hline Large Earth case (locked) & 0.65 & 4.15 & 150 \\
\hline Ocean Planet & 0.37 & 2.80 & 150 \\
\hline
\end{tabular}

Table 7.1: Magnetospheric parameters assumed for the calculation of the cosmic ray flux.

Fig. 7.3 compares the impact area obtained for the "locked case" (dashed line) with the corresponding result for the "Earth case" (solid line). Note that the dashed curve corresponds to the situation of Fig. 7.2. The following statements can be made: The impact area is much larger for the "locked case" than for the "Earth case". Also, the minimum energy required to have cosmic ray particles at any latitude (corresponding to an impact area equal to unity) is strongly reduced in the "locked case".

Fig. 7.4 shows the cosmic ray energy spectrum calculated for the two planets. It can be seen that for all energies, the cosmic ray flux to the atmosphere of a tidally locked planet (dashed line) is higher than that at Earth (solid line) by at least a factor of two. At lower energies the difference is even more pronounced, and reaches values of one order of magnitude and more for energies below $200 \mathrm{MeV}$. Comparison with the energy spectrum outside the magnetosphere (dash-dotted line) shows that the magnetospheric protection is much weaker for the tidally locked planet than it is at Earth. For energies above $2 \mathrm{GeV}$ magnetospheric shielding is negligible for the "locked case" planets. At the same time, the dash-dotted curve can be regarded as the energy spectrum at the top of the atmosphere of a totally unmagnetised planet.

\subsubsection{Influence of tidal locking}

Both the reduced planetary magnetic dipole moment due to tidal locking (Section 4.6) and the enhanced stellar wind ram pressure at small orbital distances (Section 5.1) contribute to the magnetospheric compression. This, in turn, determines the flux of high energy cosmic ray particles into the planetary atmosphere. Here, the relative importance of these two effects is compared.

The dependence of the impact area on the particle energy is shown in Fig. 7.5. When comparing the "unlocked case" (triangles) and the "Earth case" (solid line), no significant difference can be found between the two datasets. This indicates that the compression of the magnetosphere by the increased stellar wind ram pressure does not influence the influx of galactic cosmic rays. Rather, the reduced magnetic moment seems to be the decisive factor.

To further verify this result, the cosmic ray energy spectra have to be compared. This is 
done in Fig.7.6. As for the impact area, the comparison of the "unlocked case" (triangles) with the "Earth case" (solid line) shows that the reduced magnetic moment (and not the stronger stellar wind) is the decisive factor for the increased influx of cosmic ray particles.

This behaviour can be partially explained by the magnetospheric compression. Table 7.1 shows that $R_{\mathrm{s}}$ is much smaller for the "locked case" than for the "unlocked case". When this is compared to the corresponding result for the "Earth case", it becomes clear that relatively similar results for the cosmic ray flux can be expected for the two latter cases. Figures 7.5 and 7.6 show that the results are not only similar, but virtually undistinguishable. The reason is that in addition to the size of the magnetosphere, also the magnetic field at the magnetopause is different for the respective cases, see Table 7.1. For the "unlocked case" the smaller size of the magnetosphere is compensated by a larger magnetic field at the magnetopause, which enhances the cosmic ray shielding.

\subsubsection{Influence of the stellar system age}

In Section 5.1, the dependence of the stellar wind parameters (density and velocity) on the age of the stellar system was discussed. The resulting compression of the magnetospheres of terrestrial exoplanets was described in Section 5.3.4. In this section, the influence this has on the cosmic ray distribution at the top of the planetary atmosphere will be studied.

First it should be noted that only time variations of the stellar wind are considered. Parameters that are kept constant include the planetary magnetic moment $\mathcal{M}$, the orbital distance $d$, the stellar mass $M_{\star}$, and the stellar radius $R_{\star}$. Also, the effect of the timedependent stellar wind on the size and shape of the astrosphere is not included. Especially at energies below $1 \mathrm{GeV}$ (Scherer et al. 2002), the cosmic ray flux is influenced by the astrosphere, so that the results given in this section represent only a first step towards a more complete and precise description.

Similarly to Fig. 7.3 , Figure 7.7 shows the impact area, comparing the results for Earth (solid line), a tidally locked planet at $0.2 \mathrm{AU}$ around a K/M star of an age of $4.6 \mathrm{Gyr}$ (dashed line), and the same planet but for a much younger star of $0.7 \mathrm{Gyr}$ (dashed line with symbols). Note that the first two cases were already presented in section 7.3.1. It can be seen that the magnetospheric compression resulting from the fast and dense stellar wind emanating from a young star leads to much higher impact areas for particle energies around $250 \mathrm{MeV}$.

Fig.7.8 again shows the cosmic ray spectrum. Here, the influence of the stellar system age can be seen, especially for energies below $1 \mathrm{GeV}$, where the number of particles reaching the atmosphere is doubled with respect to the $4.6 \mathrm{Gyr}$ old stellar system. For a stellar system of $0.7 \mathrm{Gyr}$ age, the comparison of the energy spectra outside (dash-dotted line) and inside (dashed line with symbols) the magnetosphere shows that magnetospheric shielding is virtually absent for tidally locked planets in the habitable zone of a young $\mathrm{K} / \mathrm{M}$ star of $0.5 M_{\odot}$.

\subsubsection{Influence of the type of planet}

For non Earth-like planets, it was shown that the planetary magnetic dipole moment and the size of the magnetosphere differ from the Earth-like case (see Table 7.1). For the "Large Earth case" the expected magnetic moment is larger than for an Earth-like planet. 
Because the planetary radius is larger, however, the standoff distance measured in planetary radii is comparable, so that the protection from galactic cosmic rays should not be expected to be much larger than for an Earth-like planet. For the "Ocean Planet case", the standoff distance measured in planetary radii is much smaller than for the Earth like "locked case", so that the cosmic ray flux through the magnetosphere can be expected to be considerably larger.

Both these expectations are confirmed. The impact area (shown in Figure 7.9) and the cosmic ray energy spectrum (Figure 7.10) demonstrate that more cosmic ray particles reach the atmosphere for the "Ocean Planet case" (dotted lines) than for the "locked case" (dashed lines), and more particles impact for the "locked case" than for the "Large Earth case" (double-dashed line). However, the differences are relatively small. At least for the planets chosen for this comparison the planetary type does not have a large influence on the efficiency of magnetospheric cosmic ray protection.

\subsection{Implications for habitability}

The results obtained in Section 7.3 indicate that a major part of the atmospheric surface area of a tidally locked extrasolar planet with an orbit inside the close-in habitable zone of a low mass star will be strongly affected by secondary cosmic rays. Both the surface fraction affected by galactic cosmic rays and the flux of energetic protons are much higher than those found at Earth. This result appears to be independent of the stellar system age (Section 7.3.3) and of the planetary composition (Section 7.3.4).

While a quantitative treatment is not yet available, it is clear that the increased flux of galactic cosmic rays at the top of the planetary atmosphere has implications for the flux of cosmic rays at the planetary surface. When galactic cosmic rays of sufficiently high energy reach the planetary atmosphere, they generate showers of secondary cosmic rays. Of the different components of these cosmic ray showers, slow neutrons have the strongest influence on biological systems. For Earth, the minimum energy which a proton must have to initiate a nuclear interaction that may be detectable at sea level is approximately $450 \mathrm{MeV}$ (Reeves et al. 1992, Shea and Smart 2000). For the planetary situations of Section 7.3, the large increase of secondary cosmic rays at the planetary surface corresponding to the increase of primary cosmic ray flux is expected to produce multiple distortions in living systems.

Biological effects, namely an increase of cell fusion indices in different cell-lines, were found to be significantly correlated with the neutron count rate at the Earth's surface (Belisheva et al. 2005). Because these experiments were undertaken during quiet solar conditions (August 1990), the change in the neutron count rate is either caused by a variation of the atmospheric pressure (which changes the efficiency of atmospheric shielding) or by a variation in the galactic cosmic ray flux outside the Earth's magnetosphere. Similar, but much stronger and more diverse effects were observed during large solar particle events, where solar cosmic rays dominate over galactic cosmic rays (see discussion in Grießmeier et al. 2005b, 2006d).

Because tidally locked Earth-like exoplanets inside the habitable zone of $\mathrm{K}$ and $\mathrm{M}$ stars are only weakly protected against high energetic primary and secondary cosmic rays, they can be expected to experience a higher surface neutron flux and stronger biological 
effects than Earth-like planets at orbital distances of about 1 AU. For this reason, it may be more difficult for life to develop on the surface of planets around $\mathrm{K}$ and $\mathrm{M}$ stars than frequently assumed.

Of course, the effect of high energy cosmic ray particles on biological systems also depends on the composition and density of the planetary atmosphere. Terrestrial planets with dense atmospheres like Venus (100 bar surface pressure) would be shielded by the planetary atmosphere, so that no secondary radiation can reach the surface. On the other hand, for planets with thin atmospheres like Mars ( 7 mbar surface pressure), the surface would probably be totally sterilised. 


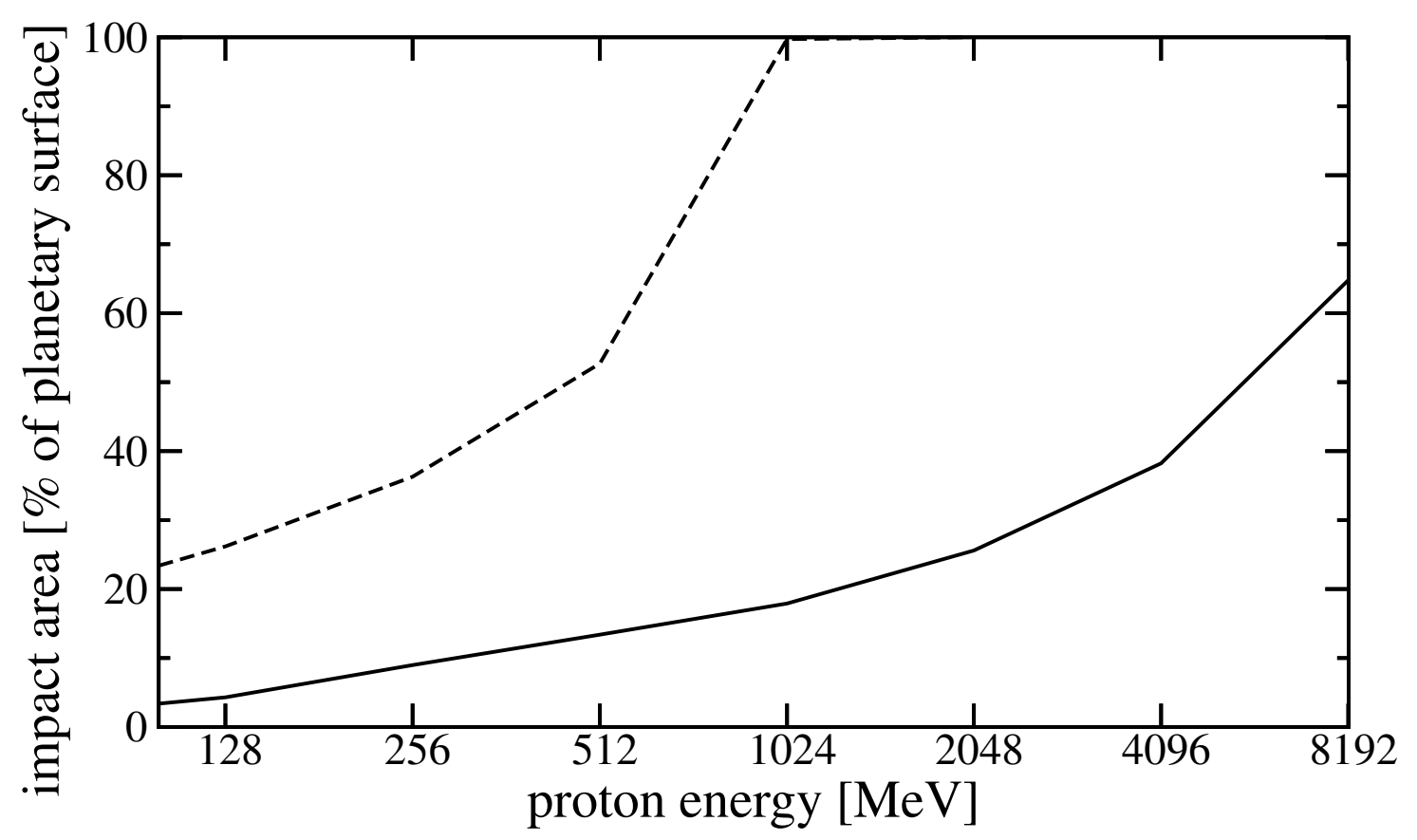

Figure 7.3: Dependence of the impact area on the energy of the cosmic ray particles. The impact area is defined as the fraction of the planetary surface where cosmic ray particles of a given energy may penetrate. Dashed line: Earth-like planet tidally locked at $0.2 \mathrm{AU}$ around a star with $M_{\star}=$ $0.5 M_{\odot}$ ("locked case"). Solid line: Earth.

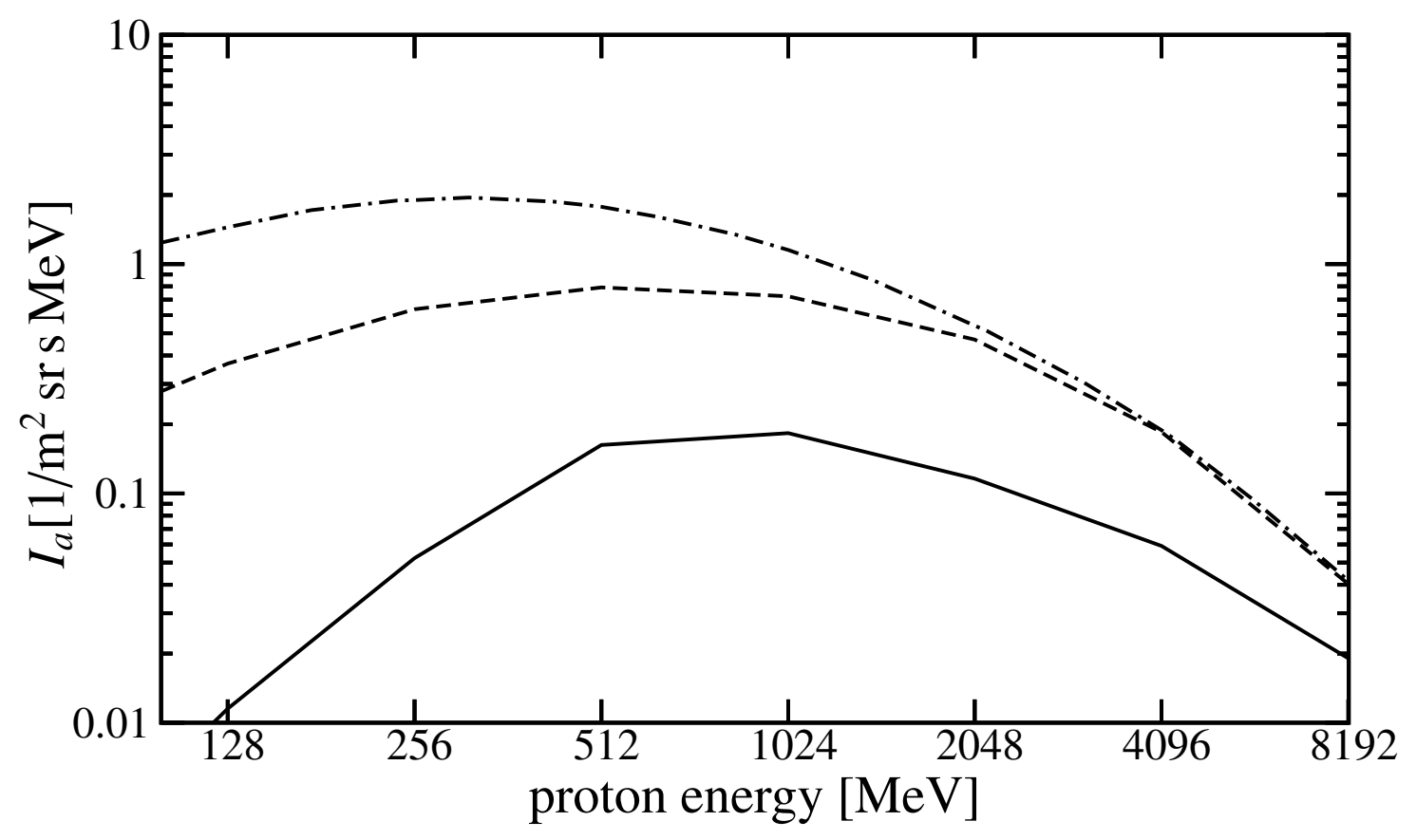

Figure 7.4: Cosmic ray energy spectrum. Dash-dotted line: energy spectrum outside the magnetosphere ("unmagnetised case"). Dashed line: Earth-like planet tidally locked at $0.2 \mathrm{AU}$ around a star with $M_{\star}=0.5 M_{\odot}$ ("locked case"). Solid line: Earth. 


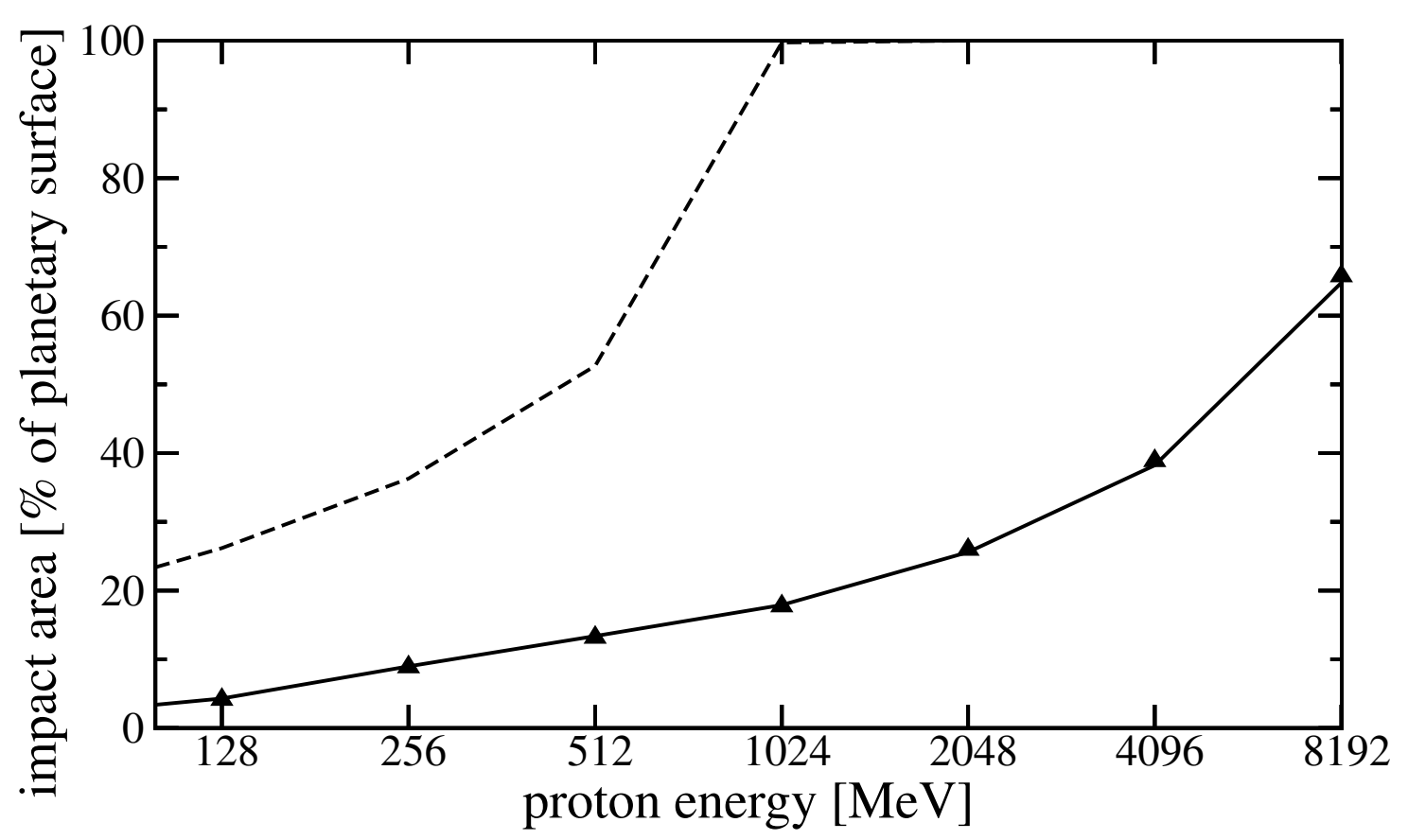

Figure 7.5: Impact area for different planets. Dashed line: "locked case". Solid line: Earth. Symbols: hypothetical "unlocked case".

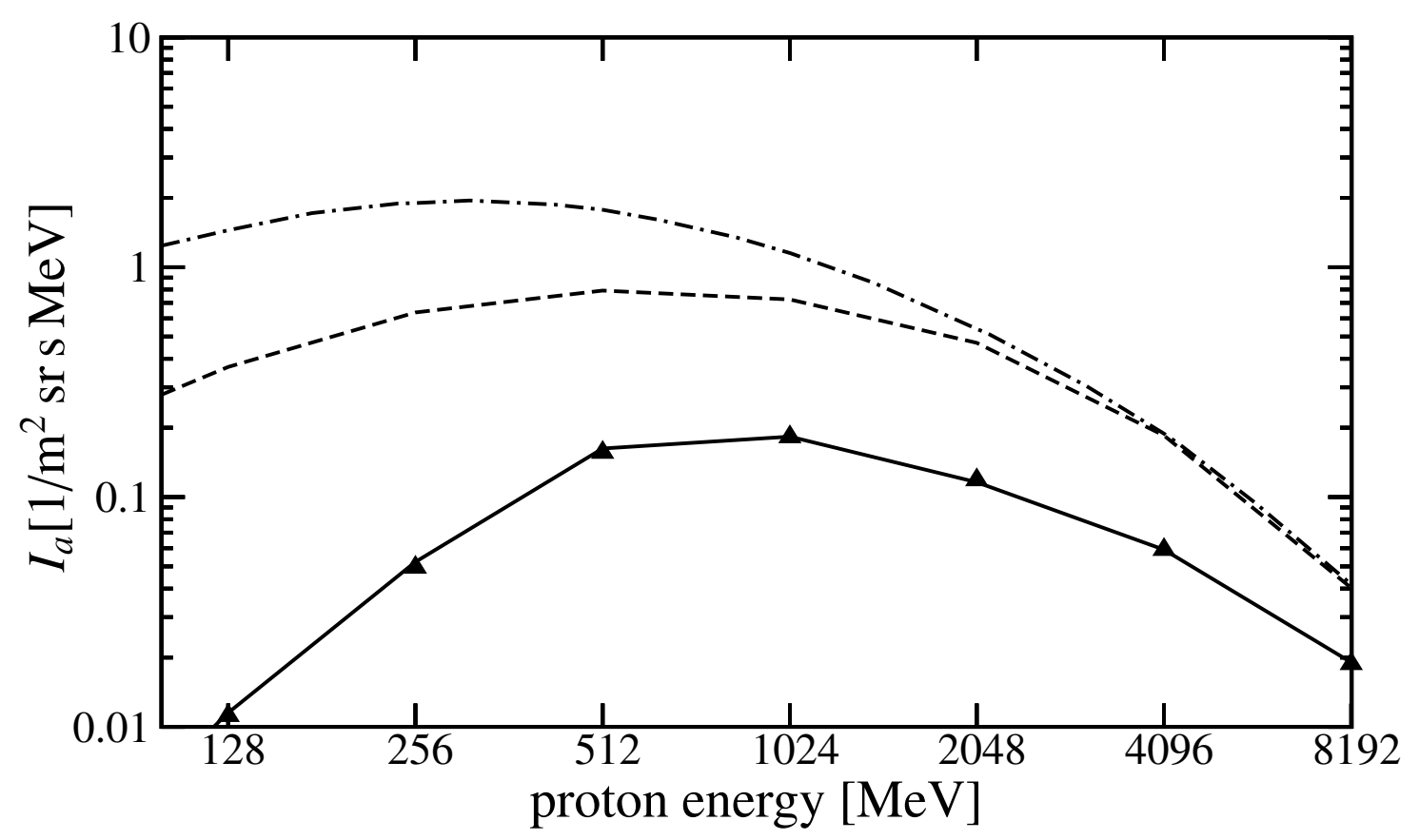

Figure 7.6: Cosmic ray energy spectrum. Dash-dotted line: energy spectrum outside the magnetosphere ("unmagnetised case"). Dashed line: "locked case". Solid line: Earth. Symbols: hypothetical "unlocked case". 


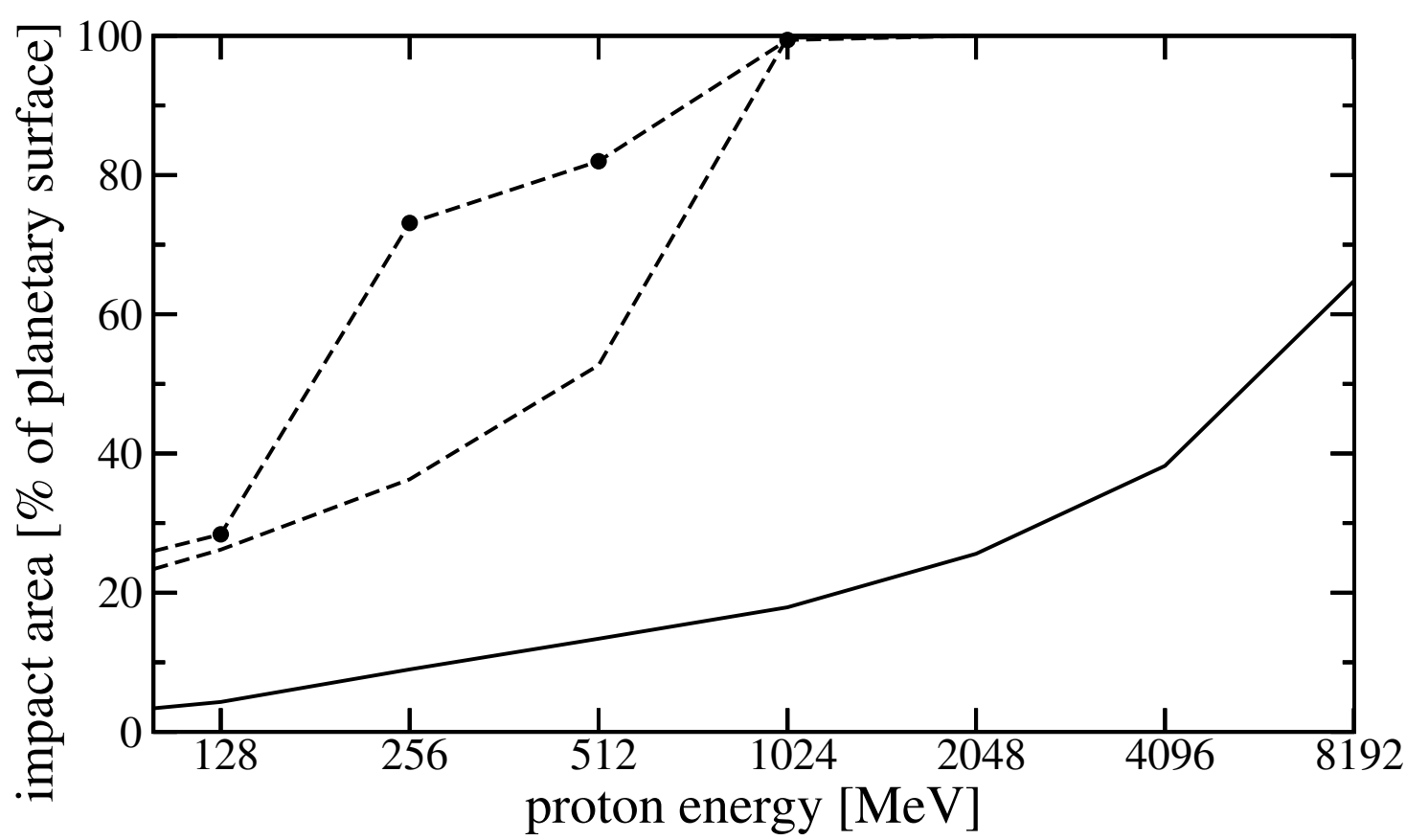

Figure 7.7: Impact area for different stellar system ages. Dashed line with symbols: planet around a star of 0.7 Gyr age ("young star case"). Dashed line without symbols: "locked case". Solid line: Earth.

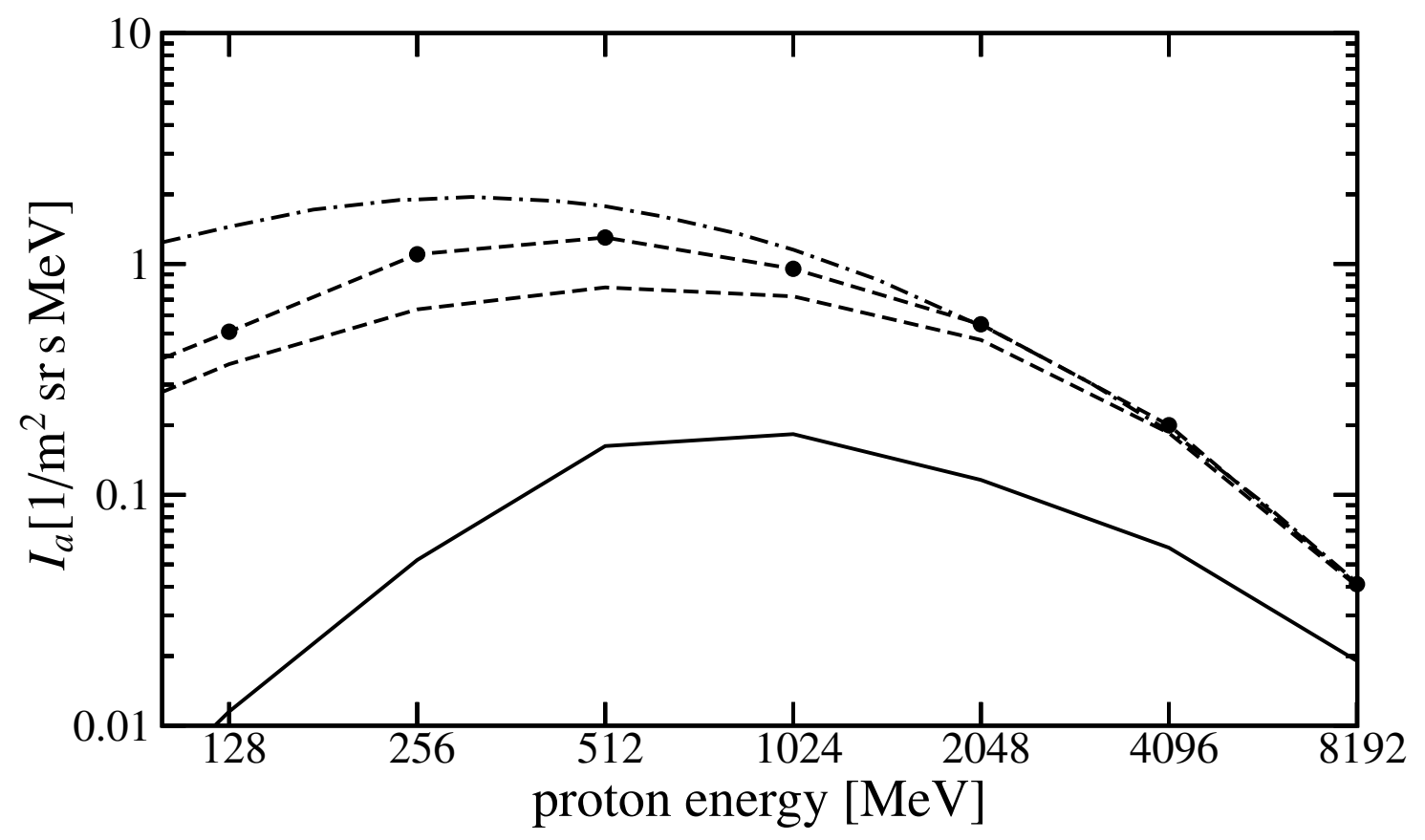

Figure 7.8: Cosmic ray energy spectrum for different stellar system ages. Dash-dotted line: energy spectrum outside the magnetosphere ("unmagnetised case"). Dashed line with symbols: planet around a star of 0.7 Gyr age ("young star case"). Dashed line without symbols: "locked case". Solid line: Earth. 


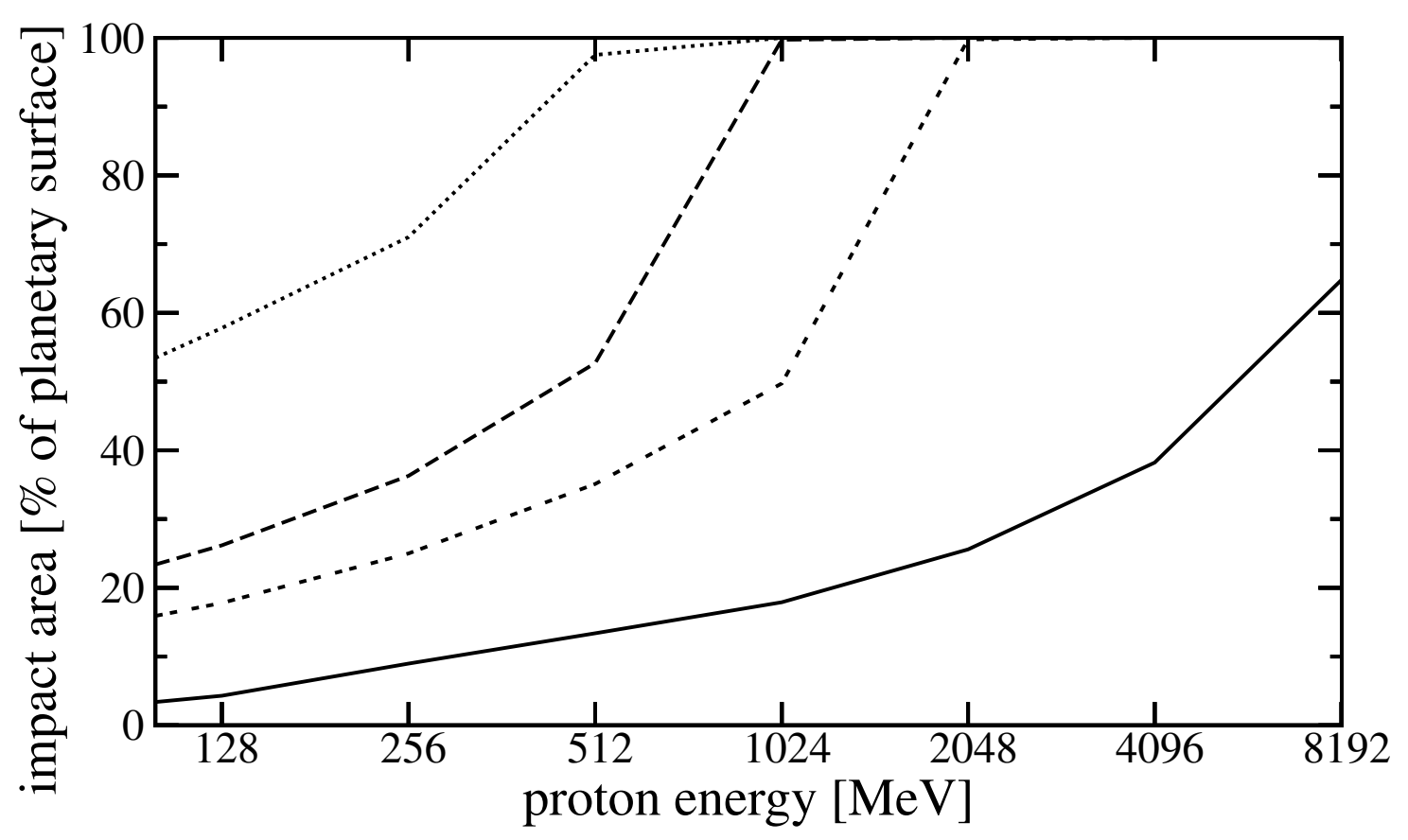

Figure 7.9: Impact area for different types of planets. Dotted line: "Ocean planet case". Dashed line: Earth-like planet ("locked case"). Double-dashed line: "Large Earth case". Solid line: Earth.

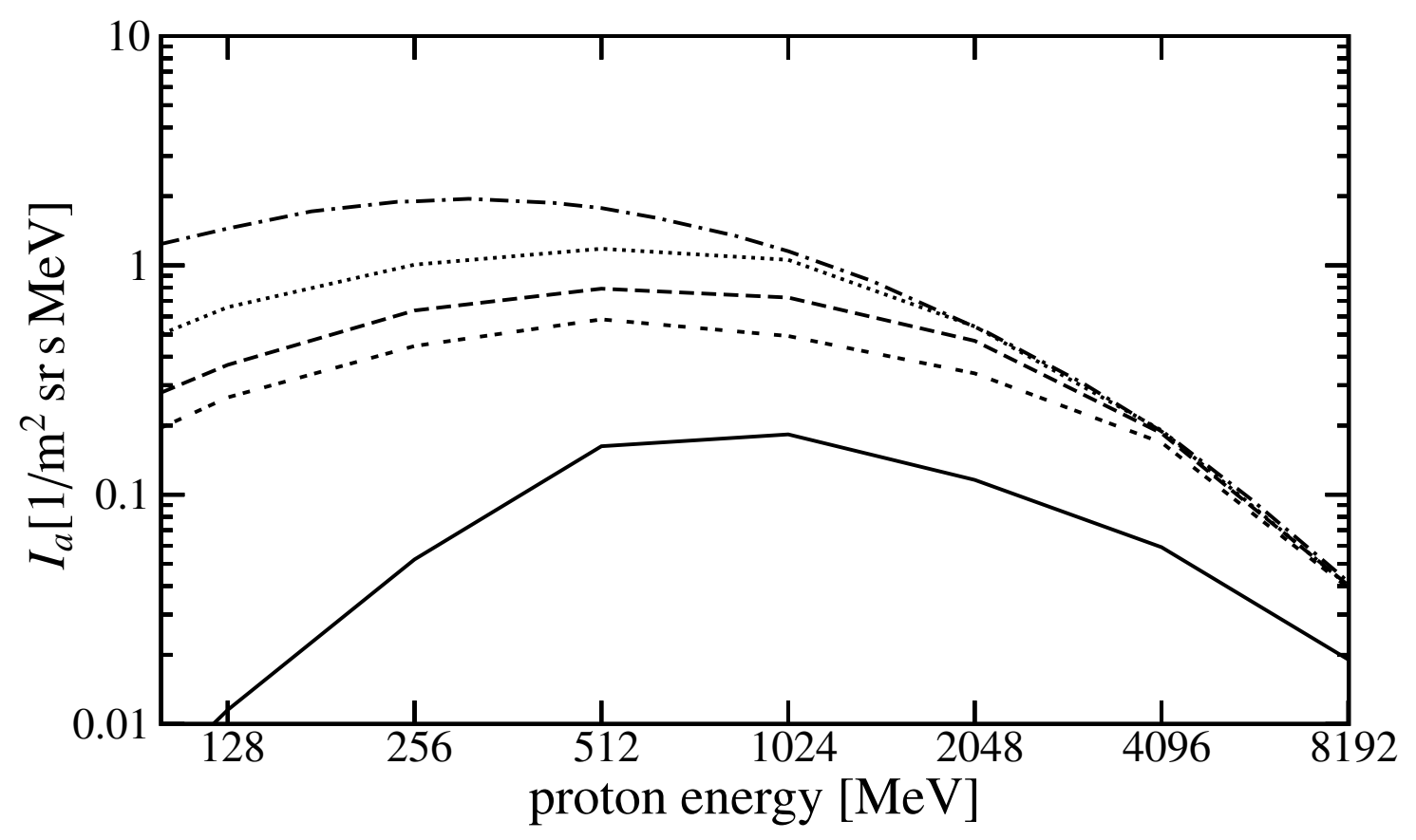

Figure 7.10: Cosmic ray energy spectrum for different for different types of planets. Dash-dotted line: energy spectrum outside the magnetosphere ("unmagnetised case"). Dotted line: "Ocean planet case". Dashed line: Earth-like planet ("locked case"). Double-dashed line: "Large Earth case". Solid line: Earth. 


\title{
8 Conclusions
}

\author{
So eine Arbeit wird eigentlich nie fertig, \\ man muß sie für fertig erklären, wenn man \\ nach Zeit und Umständen das möglichste \\ getan hat. \\ Johann Wolfgang von Goethe, \\ Italienische Reise. Caserta, den 16. März (1787)
}

In the last ten years, 170 extrasolar planets were detected. Many of them have orbital distances much smaller than any of the planets in our solar system. This results in a different stellar flux at the location of the planet, in a denser stellar wind environment, and leads to strong tidal interaction. This remarkable environment gives rise to several questions concerning, for example, the influence of tidal locking on the planetary magnetic moment, the interaction between the dense stellar wind and the planetary magnetic field, the magnitude of atmospheric escape, the intensity of magnetospheric radio emission, and the shielding of the planet against cosmic rays.

In this work, some of these questions are addressed, showing that small orbital distances have important consequences for the planets and for their magnetospheres. Fig. 1.1 schematically shows the interrelation between the most relevant effects.

At small enough orbital distances, tidal interaction leads to tidal locking. For example, all Hot Jupiters with orbital radii of $d \lesssim 0.08$ AU around solar-mass stars can be considered as tidally locked. Tidal locking is also important for most terrestrial exoplanets orbiting within the habitable zone of $\mathrm{M}$ stars. The critical distance for orbital circularisation is considerably smaller: For Hot Jupiters around Sun-like stars, circularised orbits can be assumed only if $d \leq 0.03$ AU. Hot Jupiters in circularised orbits also have zero obliquity. For Earth-like exoplanets, the critical distance for orbital circularisation is slightly larger than for Hot Jupiters, but planets within the habitable zone of M stars can still have eccentric orbits and nonzero obliquity.

Using different analytical scaling laws it is shown that, both for gaseous giant planets and for terrestrial planets, the planetary magnetic moment is strongly reduced by tidal locking. Even for planets considerably larger than Jupiter, magnetic moments are not likely to be much larger than that of Jupiter. For more Jupiter-like planets, the planetary magnetic moment can be much smaller (between 0.024 and 0.71 times the magnetic moment of Jupiter). Similarly, tidally locked terrestrial exoplanets in the habitable zone of M stars have much smaller magnetic moments than Earth. For example, for an Earth-like exoplanet at $0.2 \mathrm{AU}$ around an M star, the maximum magnetic moment is $15 \%$ of the magnetic moment of the Earth.

To evaluate the influence of the stellar wind, the stellar wind conditions at close orbital 
distances have to be calculated. For stars with ages of at least $0.7 \mathrm{Gyr}$, the stellar wind model of Parker (1958) can be used to determine the distance dependence of the stellar wind. Also, the temporal evolution of the stellar wind density, velocity and temperature is taken into account, so that stellar winds of stars with different ages ( $\geq 0.7 \mathrm{Gyr})$ can be modelled. For young stars, one finds that the stellar wind velocity is higher by a factor of two, and the stellar wind density is higher by one order of magnitude when compared to a star having the same age as the Sun. In addition, the role of stellar CMEs (as analogues to solar coronal mass ejections) is considered. For stars with high CME activity, the stellar wind is effectively replaced by a stream of CMEs, for which the density and velocity are approximately comparable to the stellar wind of a young star.

Because of the denser stellar wind and the reduction of the planetary magnetic moment by tidal locking, the magnetospheres of close-in exoplanets are much smaller than the magnetospheres of more distant planets. Depending on the planetary magnetic moment and the stellar wind parameters, magnetospheric standoff distances can be as large as 7 planetary radii, but in some cases, standoff distances comparable to the size of the planetary radius are found.

With the magnetosphere thus determined, two different applications are discussed. First, the increased stellar wind density is responsible for a high energy flux to planetary magnetospheres. This results in an enhancement of planetary radio emission from gaseous giant exoplanets. Compared to Jupiter, which is the strongest planetary radio emitter presently known, the radio power of the planet $\tau$ Bootes $\mathrm{b}$ is expected to be larger by four orders of magnitude. At the same time, such planetary emission is likely to be stronger than the radio emission of the host star, which will facilitate detection. For $\tau$ Bootes $\mathbf{b}$, the maximum emission frequency is $18 \mathrm{MHz}$, and the radio flux expected at Earth is of the order of $40 \mathrm{mJy}\left(1 \mathrm{Jy}=10^{-26} \mathrm{~W} \mathrm{~m}^{-2} \mathrm{~Hz}^{-1}\right)$. The frequency range and the sensitivity of current radio detectors are not sufficient to detect such emission, which is the reason for the current non-detections. With planned modifications of the existing instrumentation and with the construction of new radio arrays, however, it seems likely that the detection of exoplanetary radio emission can be used as an additional observation method in the near future.

While so far no Earth-sized exoplanets have been detected, such a detection is likely to occur in the near future. The Earth's magnetosphere is known to play an important role in the protection of the planetary surface against the impact of high-energy cosmic ray particles. Because all extrasolar planets with orbits inside the habitable zone of $\mathrm{M}$ stars are subject to tidal locking, the question arises as to whether the same degree of protection can be expected for terrestrial extrasolar planets. This question is studied for different types of terrestrial planets. It is shown that a strong magnetic moment and a large magnetosphere are important for the protection of the planet against galactic cosmic rays. Planets within the habitable zone of $\mathrm{M}$ stars do not fulfil these requirements. For this reason, it may be more difficult for life to develop on the surface of planets around M stars than previously assumed. More work is required to better evaluate whether $\mathrm{M}$ stars are good targets in the search for habitable planets. This is important, for example, when selecting targets for the DARWIN mission (Fridlund 2004), which will search for biosignatures in the spectra of terrestrial exoplanets. The weak protection of planets around $\mathrm{M}$ stars against galactic cosmic rays implies that it has to be assured that enough non-M stars are in the sample to maximise the likelihood of a positive detection. 


\section{Bibliography}

Alonso, R., Brown, T. M., Torres, G., Latham, D. W., Sozzetti, A., Mandushev, G., Belmonte, J. A., Charbonneau, D., Deeg, H. J., Dunham, E. W., O’Donovan, F. T., Stefanik, R. P., 2004, TrES-1: The transiting planet of a bright K0 V star, Astrophys. J., 613, L153-L156

Arge, C. N., Mullan, D. J., Dolginov, A. Z., 1995, Magnetic moments and angular momenta of stars and planets, Astrophys. J., 443, 795-803

Atobe, K., Ida, S., Ito, T., 2004, Obliquity variations of terrestrial planets in habitable zones, Icarus, 168, 223-236

Bains, W., 2004, Many chemistries could be used to build living systems, Astrobiology, 4, 137-167

Baliunas, S., Sokoloff, D., Soon, W., 1996, Magnetic field and rotation in lower mainsequence stars: an empirical time-dependent magnetic Bode's relation?, Astrophys. J., 457, L99-L102

Barnes, J. W., O’Brien, D. P., 2002, Stability of satellites around close-in extrasolar giant planets, Astrophys. J., 575, 1087-1093

Barrow, C. H., Desch, M. D., 1989, Solar wind control of Jupiter's hectometric radio emission, Astron. Astrophys., 213, 495-501

Basri, G., Brown, M. E., 2006, Planetesimals to brown dwarfs: What is a planet?, Annu. Rev. Earth. Planet. Sci., 34, 193-216

Bastian, T. S., Dulk, G. A., Leblanc, Y., 2000, A search for radio emission from extrasolar planets, Astrophys. J., 545, 1058-1063

Baumjohann, W., Treumann, R. A., 1999, Basic Space Plasma Physics, Imperial College Press, London

Belisheva, N. K., Kuzhevskii, B. M., Vashenyuk, E. V., Zhirov, V. K., 2005, Correlation between the fusion dynamics of cells growing in vitro and variations of neutron intensity near the Earth's surface, Doklady Biochemistry and Biophysics, 402, 254-257

Benedict, G. F., McArthur, B. E., Forveille, T., Delfosse, X., Nelan, E., Butler, R. P., Spiesman, W., Marcy, G., Goldman, B., Perrier, C., Jefferys, W. H., Mayor, M., 2002, A mass for the extrasolar planet Gliese $876 \mathrm{~b}$ determined from Hubble Space Telescope 
fine guidance sensor 3 astrometry and high-precision radial velocities, Astrophys. J., 581, L115-L118

Benz, A., 1993, Plasma Astrophysics: Kinetic Processes in Solar and Stellar Coronae, Kluwer Academic Publishers, Dordrecht

Blackett, P. M. S., 1947, The magnetic field of massive rotating bodies, Nature, 159, 658-666

Blackett, P. M. S., 1952, A negative experiment relating to magnetism and the Earth's rotation, Phil. Trans. R. Soc. A, 245, 309-370

Bodenheimer, P., Laughlin, G., Lin, D. N. C., 2003, On the radii of extrasolar giant planets, Astrophys. J., 592, 555-563

Boischot, A., Denisse, J. F., 1964, Solar radio astronomy, Adv. in Electronics and Electron. Phys., 20, 147-198

Bond, I. A., Udalski, A., Jaroszyński, M., Rattenbury, N. J., Paczyński, B., Soszyński, I., Wyrzykowski, L., Szymański, M. K., Kubiak, M., Szewczyk, O., Żebruń, K., Pietrzyński, G., Abe, F., Bennett, D. P., Eguchi, S., Furuta, Y., Hearnshaw, J. B., Kamiya, K., Kilmartin, P. M., Kurata, Y., Masuda, K., Matsubara, Y., Muraki, Y., Noda, S., Okajima, K., Sako, T., Sekiguchi, T., Sullivan, D. J., Sumi, T., Tristram, P. J., Yanagisawa, T., Yock, P. C. M., 2004, OGLE 2003-BLG-235/MOA 2003-BLG-53: A planetary microlensing event, Astrophys. J., 606, L155-L158

Bordé, P., Rouan, D., Léger, A., 2003, Exoplanet detection capability of the COROT space mission, Astron. Astrophys., 405, 1137-1144

Bouchy, F., Pont, F., Santos, N. C., Melo, C., Mayor, M., Queloz, D., Udry, S., 2004, Two new "very hot Jupiters" among the OGLE transiting candidates, Astron. Astrophys., 421, L13-L16

Bouchy, F., Pont, F., Melo, C., Santos, N. C., Mayor, M., Queloz, D., Udry, S., 2005, Doppler follow-up of OGLE transiting companions in the Galactic bulge, Astron. Astrophys., 431, 1105-1121

Bougeret, J.-L., King, J. H., Schwenn, R., 1984, Solar radio burst and in situ determination of interplanetary electron density, Solar Phys., 90, 401-412

Brandt, J. C., 1970, Introduction to the Solar Wind, W. H. Freeman and Company, San Francisco

Burke, B. F., 1992, Prospects for the study of planetary radio emission, in Planetary Radio Emissions III, (Eds.) H. O. Rucker, S. J. Bauer, M. L. Kaiser, pp. 485-488, Austrian Academy of Sciences Press, Vienna

Burke, B. F., Franklin, K. L., 1955, Observations of a variable radio source associated with the planet Jupiter, J. Geophys. Res., 60, 213-217 
Burkert, A., Lin, D. N. C., Bodenheimer, P. H., Jones, C. A., Yorke, H. W., 2005, On the surface heating of synchronously spinning short-period jovian planets, Astrophys. J., $618,512-523$

Burrows, A., Angel, R., 1999, Direct detection at last, Nature, 402, 732-733

Burrows, A., Marley, M., Hubbard, W. B., Lunine, J. I., Guillot, T., Saumon, D., Freedman, R., Sudarsky, D., Sharp, C., 1997, A nongray theory of extrasolar giant planets and brown dwarfs, Astrophys. J., 491, 856-875

Burrows, A., Guillot, T., Hubbard, W. B., Marley, M. S., Saumon, D., Lunine, J. I., Sudarsky, D., 2000, On the radii of close-in giant planets, Astrophys. J., 534, L97L100

Burrows, A., Sudarsky, D., Hubbard, W. B., 2003, A theory for the radius of the transiting giant planet HD 209458b, Astrophys. J., 594, 545-551

Burrows, A., Hubeny, I., Hubbard, W. B., Sudarsky, D., Fortney, I. J., 2004a, Theoretical radii of transiting giant planets: the case of OGLE-TR-56b, Astrophys. J., 610, L53L56

Burrows, A., Sudarsky, D., Hubeny, I., 2004b, Spectra and diagnostics for the direct detection of wide-separation extrasolar giant planets, Astrophys. J., 609, 407-416

Busse, F. H., 1976, Generation of planetary magnetism by convection, Phys. Earth Planet. Inter., 12, 350-358

Cain, J. C., Beaumont, P., Holter, W., Wang, Z., Nevanlinna, H., 1995, The magnetic bode fallacy, J. Geophys. Res., 100, 9439-9454

Carr, T. D., Desch, M. D., Alexander, J. K., 1983, Phenomenology of magnetospheric radio emissions, in Physics of the Jovian Magnetosphere, (Ed.) A. J. Dessler, chap. 7, pp. 226-284, Cambridge Univ. Press, Cambridge

Chabrier, G., Baraffe, I., 1997, Structure and evolution of low-mass stars, Astron. Astrophys., 327, 1039-1053

Chandrasekhar, S., 1957, An Introduction to the Study of Stellar Structure, Dover Books on Astronomy and Astrophysics, Dover Publications, Inc., New York

Charbonneau, D., 2004, A review of the current status of follow-up techniques to study known extrasolar planets, in Stars as Suns: Activity, Evolution, and Planets, (Eds.) A. K. Dupree, A. O. Benz, vol. 219 of IAU Symposium, pp. 367-378

Charbonneau, D., Brown, T. M., Latham, D. W., Mayor, M., 2000, Detection of planetary transits across a sun-like star, Astrophys. J., 529, L45-L48

Charbonneau, D., Brown, T. M., Noyes, R. W., Gilliland, R. L., 2002, Detection of an extrasolar planet atmosphere, Astrophys. J., 568, 377-384 
Charbonneau, D., Allen, L. E., Megeath, S. T., Torres, G., Alonso, R., Brown, T. M., Gilliland, R. L., Latham, D. W., Mandushev, G., O’Donovan, F. T., Sozzetti, A., 2005, Detection of thermal emission from an extrasolar planet, Astrophys. J., 626, 523-529

Chauvin, G., Lagrange, A.-M., Dumas, C., Zuckerman, B., Mouillet, D., Song, I., Beuzit, J.-L., Lowrance, P., 2004, A giant planet candidate near a young brown dwarf. Direct VLT/NACO observations using IR wavefront sensing, Astron. Astrophys., 425, L29L32

Chauvin, G., Lagrange, A.-M., Dumas, C., Zuckerman, B., Mouillet, D., Song, I., Beuzit, J.-L., Lowrance, P., 2005a, Giant planet companion to 2MASSW J1207334-393254, Astron. Astrophys., 438, L25-L28

Chauvin, G., Lagrange, A.-M., Lacombe, F., Dumas, C., Mouillet, D., Zuckerman, B., Gendron, E., Song, I., Beuzit, J.-L., Lowrance, P., Fusco, T., 2005b, Astrometric and spectroscopic confirmation of a brown dwarf companion to GSC 08047-00232, Astron. Astrophys., 430, 1027-1033

Christensen, U. R., Aubert, J., 2006, Scaling properties of convection-driven dynamos in rotating spherical shells and application to planetary magnetic fields, Geophys. J. Int., $166,97-114$

Cody, A. M., Sasselov, D. D., 2002, HD 209458: Physical parameters of the parent star and the transiting planet, Astrophys. J., 569, 451-458

Collier Cameron, A., 2002, What are hot Jupiters made of?, Astronomy and Geophysics, $43,4.21-4.25$

Connerney, J. E. P., 1993, Magnetic fields of the outer planets, J. Geophys. Res., 98, $18659-18679$

Correia, A. C. M., Laskar, J., 2001, The four final rotation states of Venus, Nature, 411, 767-770

Correia, A. C. M., Laskar, J., 2003a, Different tidal torques on a planet with a dense atmosphere and consequences to the spin dynamics, J. Geophys. Res., 108, 9-1-9-10

Correia, A. C. M., Laskar, J., 2003b, Long-term evolution of the spin of Venus II. Numerical simulations, Icarus, 163, 24-45

Correia, A. C. M., Laskar, J., de Surgy, O. N., 2003, Long-term evolution of the spin of Venus I. Theory, Icarus, 163, 1-23

Cuntz, M., Shkolnik, E., 2002, Chromospheres, flares and exoplanets, Astron. Nachr., $323,387-391$

Cuntz, M., Saar, S. H., Musielak, Z. E., 2000, On stellar activitity enhancement due to interactions with extrasolar giant planets, Astrophys. J., 533, L151-154

Curtis, S. A., Ness, N. F., 1986, Magnetostrophic balance in planetary dynamos: Predictions for Neptune's magnetosphere, J. Geophys. Res., 91, 11 003-11 008 
Deming, D., Seager, S., Richardson, L. J., Harrington, J., 2005, Infrared radiation from an extrasolar planet, Nature, 434, 740-743

Desch, M. D., 1988, Neptune radio emission: predictions based on planetary scaling laws, Geophys. Res. Lett., 15, 114-117

Desch, M. D., Barrow, C. H., 1984, Direct evidence for solar wind control of Jupiter's hectometer-wavelength radio emission, J. Geophys. Res., 89, 6819-6823

Desch, M. D., Kaiser, M. L., 1984, Predictions for Uranus from a radiometric Bode's law, Nature, 310, 755-757

Desch, M. D., Rucker, H. O., 1983, The relationship between Saturn kilometric radiation and the solar wind, J. Geophys. Res., 88, 8999-9006

Dobbs-Dixon, I., Lin, D. N. C., Mardling, R. A., 2004, Spin-orbit evolution of shortperiod planets, Astrophys. J., 610, 464-476

Dorren, J. D., Guinan, E. F., 1994, HD 129333: The sun in its infancy, Astrophys. J., 428, 805-818

Dougherty, M. K., Achilleos, N., Andre, N., Arridge, C. S., Balogh, A., Bertucci, C., Burton, M. E., Cowley, S. W. H., Erdos, G., Giampieri, G., Glassmeier, K.-H., Khurana, K. K., Leisner, J., Neubauer, F. M., Russell, C. T., Smith, E. J., Southwood, D. J., Tsurutani, B. T., 2005, Cassini magnetometer observations during Saturn orbit insertion, Science, 307, 1266-1270

Eltayeb, I. A., Roberts, P. H., 1970, On the hydromagnetics of rotating fluids, Astrophys. J., 162, 699-701

Ergun, R. E., Carlson, C. W., McFadden, J. P., Delory, G. T., Strangeway, R. J., Pritchett, P. L., 2000, Electron-cyclotron Maser driven by charged-particle acceleration from magnetic field-aligned electric fields, Astrophys. J., 538, 456-466

Erkaev, N. V., Penz, T., Lammer, H., Lichtenegger, H. I. M., Biernat, H. K., Wurz, P., Grießmeier, J.-M., Weiss, W. W., 2005, Plasma and magnetic field parameters in the vicinity of short-periodic giant exoplanets, Astrophys. J. Suppl. Ser., 157, 396-401

Farrell, W. M., Desch, M. D., Zarka, P., 1999, On the possibility of coherent cyclotron emission from extrasolar planets, J. Geophys. Res., 104, 14 025-14 032

Farrell, W. M., Desch, M. D., Lazio, T. J., Bastian, T., Zarka, P., 2003, Limits on the magnetosphere/stellar wind interactions for the extrasolar planet about Tau Bootes, in Scientific Frontiers in Research of Extrasolar Planets, (Eds.) D. Deming, S. Seager, vol. 294 of ASP Conference Series, pp. 151-155

Farrell, W. M., Lazio, T. J. W., Zarka, P., Bastian, T. J., Desch, M. D., Ryabov, B. P., 2004, The radio search for extrasolar planets with LOFAR, Planet. Space Sci., 52, 1469-1478 
Fortney, J. J., Marley, M. S., Lodders, K., Saumon, D., Freedman, R., 2005, Comparative planetary atmospheres: Models of TrES-1 and HD 209458B, Astrophys. J., 627, L69L72

Franck, S., von Bloh, W., Bounama, C., Steffen, M., Schönberner, D., Schellnhuber, H.J., 2000, Determination of habitable zones in extrasolar planetary systems: Where are Gaia's sisters?, J. Geophys. Res., 105, 1651-1658

Franck, S., Cuntz, M., von Bloh, W., Bounama, C., 2003, The habitable zone of Earthmass planets around 57 UMa: results for land and water worlds, Int. J. Astrobiol., 2, 35-39

Fridlund, C. V. M., 2004, The Darwin mission, Adv. Space Res., 34, 613-617

Fuhrmann, K., Pfeiffer, M. J., Bernkopf, J., 1998, F- and G-type stars with planetary companions: $\nu$ Andromedae, $\rho^{1}$ Cancri, $\tau$ Bootis, 16 Cygni and $\rho$ Coronae Borealis, Astron. Astrophys., 336, 942-952

Gallagher, D. L., D’Angelo, N., 1981, Correlations between solar wind parameters and auroral kilometric radiation intensity, Geophys. Res. Lett., 8, 1087-1089

Gaudi, B. S., Han, C., 2004, The many possible interpretations of microlensing event OGLE 2002-BLG-055, Astrophys. J., 611, 528-536

Glanz, J., 1997, Worlds around other stars shake planet birth theory, Science, 276, 13361339

Glaßmeier, K.-H., Vogt, J., 2001, Physik des Erdkörpers, Manuskript zur Vorlesung

Glassmeier, K.-H., Vogt, J., Stadelmann, A., Buchert, S., 2004, Concerning long-term geomagnetic variations and space climatology, Ann. Geophys., 22, 3669-3677

Gliese, W., Jahreiß, H., 1991, Preliminary version of the third catalogue of nearby stars, Tech. rep., On: The Astronomical Data Center CD-ROM: Selected Astronomical Catalogs, Vol. I; L.E. Brotzmann, S.E. Gesser (eds.), NASA/Astronomical Data Center, Goddard Space Flight Center, Greenbelt, MD

Goldreich, P., Soter, S., 1966, Q in the solar system, Icarus, 5, 375-389

Goukenleuque, C., Bézard, B., Joguet, B., Lellouch, E., Freedman, R., 2000, A radiative equilibrium model of 51 Peg b, Icarus, 143, 308-323

Grießmeier, J.-M., Stadelmann, A., Penz, T., Lammer, H., Selsis, F., Ribas, I., Guinan, E. F., Motschmann, U., Biernat, H. K., Weiss, W. W., 2004, The effect of tidal locking on the magnetospheric and atmospheric evolution of "Hot Jupiters", Astron. Astrophys., 425, 753-762

Grießmeier, J.-M., Motschmann, U., Mann, G., Rucker, H. O., 2005a, The influence of stellar wind conditions on the detectability of planetary radio emissions, Astron. Astrophys., 437, 717-726 
Grießmeier, J.-M., Stadelmann, A., Motschmann, U., Belisheva, N. K., Lammer, H., Biernat, H. K., 2005b, Cosmic ray impact on extrasolar Earth-like planets in close-in habitable zones, Astrobiology, 5, 587-603

Grießmeier, J.-M., Motschmann, U., Glassmeier, K.-H., Mann, G., Rucker, H. O., 2006a, The potential of exoplanetary radio emissions as an observation method, in Tenth Anniversary of 51 Peg-b : Status of and Prospects for hot Jupiter studies, (Eds.) L. Arnold, F. Bouchy, C. Moutou, pp. 259-266, Platypus Press, URL: http://www.obshp.fr/www/pubs/Coll51Peg/proceedings.html

Grießmeier, J.-M., Motschmann, U., Khodachenko, M., Rucker, H. O., 2006b, The influence of stellar coronal mass ejections on exoplanetary radio emission, in Planetary Radio Emissions VI, (Eds.) H. O. Rucker, W. S. Kurth, G. Mann, pp. 571-579, Austrian Academy of Sciences Press, Vienna

Grießmeier, J.-M., Preusse, S., Khodachenko, M., Motschmann, U., Mann, G., Rucker, H. O., 2006c, Exoplanetary radio emission under different stellar wind conditions, Planet. Space Sci., submitted

Grießmeier, J.-M., Stadelmann, A., Lammer, H., Belisheva, N., Motschmann, U., 2006d, The impact of galactic cosmic rays on extrasolar Earth-like planets in close-in habitable zones, in Proc. 39th ESLAB Symposium, (Eds.) F. Favata, A. Gimenez, pp. 305-309, ESA SP-588

Gu, P.-G., Lin, D. N. C., Bodenheimer, P. H., 2003, The effect of tidal inflation instability on the mass and dynamical evolution of extrasolar planets with ultrashort periods, Astrophys. J., 588, 509-534

Güdel, M., Benz, A. O., 1996, Radio spectra of dMe and dKe stars, in ASP Conf. Ser. 93: Radio Emission from the Stars and the Sun, (Eds.) A. R. Taylor, J. M. Paredes, pp. 303-305

Güdel, M., Zucker, A., 2000, Gyrosynchrotron emission from stellar coronae, in IAU Symp. 195: Highly Energetic Physical Processes and Mechanisms for Emission from Astrophysical Plasmas, (Eds.) P. C. H. Martens, S. Tsuruta, pp. 393-394

Güdel, M., Benz, A. O., Bastian, T. S., Fürst, E., Simnett, G. M., Davis, R. J., 1989, Broadband spectral observation of a dMe star radio flare, Astron. Astrophys., 220, L5L8

Güdel, M., Schmitt, J. H. M. M., Benz, A. O., 1995, Microwave emission from X-ray bright solar-like stars: the F-G main sequence and beyond, Astron. Astrophys., 302, 775-787

Güdel, M., Guinan, E. F., Skinner, S. L., 1998, The X-ray and radio Sun in time: Coronal evolution of solar-type stars with different ages, in ASP Conf. Ser. 154: Cool Stars, Stellar Systems and the Sun, (Eds.) R. A. Donahue, J. A. Bookbinder, pp. 1041-1050

Guillot, T., 1999, Interiors of giant planets inside and outside the solar system, Science, 286, 72-77 
Guillot, T., Showman, A. P., 2002, Evolution of "51 Pegasus b-like" planets, Astron. Astrophys., 385, 156-165

Guillot, T., Burrows, A., Hubbard, W. B., Lunine, J. I., Saumon, D., 1996, Giant planets at small orbital distances, Astrophys. J., 459, L35-L38

Guinan, E. F., Ribas, I., 2002, Our changing sun: The role of solar nuclear evolution and magnetic activity on Earth's atmosphere and climate, in The Evolving Sun and its Influence on Planetary Environments, (Eds.) B. Montesinos, A. Gimenez, E. F. Guinan, vol. 269 of ASP Conference Series, pp. 85-107

Gurnett, D. A., Kurth, W. S., Hospodarsky, G. B., Persoon, A. M., Zarka, P., Lecacheux, A., Bolton, S. J., Desch, M. D., Farrell, W. M., Kaiser, M. L., Ladreiter, H.-P., Rucker, H. O., Galopeau, P., Louarn, P., Young, D. T., Pryor, W. R., Dougherty, M. K., 2002, Control of Jupiter's radio emission and aurorae by the solar wind, Nature, 415, 985-987

Halbwachs, J. L., Mayor, M., Udry, S., 2005, Statistical properties of exoplanets IV. The period-eccentricity relations of exoplanets and of binary stars, Astron. Astrophys., 431, $1129-1137$

Heath, M. J., Doyle, L. R., Joshi, M. M., Haberle, R. M., 1999, Habitability of planets around red dwarf stars, Origins Life Evol. Biosphere, 29, 405-424

Henry, G. W., Marcy, G. W., Butler, R. P., Vogt, S. S., 2000, A transiting "51 Peg-like" planet, Astrophys. J., 529, L41-L44

Hill, T. W., 1979, Rates of mass, momentum, and energy transfer at the magnetopause, in Proceedings of Magnetospheric Boundary Layers Conference, Alpbach, pp. 325-332, ESA SP-148

Hjellming, R. M., 1988, Radio stars, in Galactic and Extragalactic Radio Astronomy, (Eds.) G. L. Verschuur, K. I. Kellermann, Astronomy and Astrophysics Library, chap. 9, pp. 381-438, Springer-Verlag, Berlin, second edn.

Holme, R., 1997, Three-dimensional kinematic dynamos with equatorial symmetry: Application to the magnetic fields of Uranus and Neptune, Phys. Earth Planet. Inter., 102, $105-122$

Hubbard, W. B., 1984, Planetary interiors, Van Nostrand Reinhold Co., New York

Hubbard, W. B., Marley, M. S., 1989, Optimized Jupiter, Saturn and Uranus interior models, Icarus, 78, 102-118

Ip, W.-H., Kopp, A., Hu, J.-H., 2004, On the magnetosphere interaction of close-in exoplanets, Astrophys. J., 602, L53-L56

Jacobs, J. A., 1979, Planetary magnetic fields, Geophys. Res. Lett., 6, 213-214

Jaritz, G. F., Endler, S., Langmayr, D., Lammer, H., Grießmeier, J.-M., Erkaev, N. V., Biernat, H. K., 2005, Roche lobe effects on expanded upper atmospheres of shortperiodic giant exoplanets, Astron. Astrophys., 439, 771-775 
Jenkins, J. M., 2002, The impact of solar-like variability on the detectability of transiting terrestrial planets, Astrophys. J., 575, 493-505

Jha, S., Charbonneau, D., Garnavich, P. M., Sullivan, D. J., Sullivan, T., Brown, T. M., Tonry, J. L., 2000, Multicolor observations of a planetary transit of HD 209458, Astrophys. J., 540, L45-L48

Jiang, I.-G., Ip, W.-H., Yeh, L.-C., 2003, On the fate of close-in extrasolar planets, Astrophys. J., 582, 449-454

Jordan, C. E., 1994, Empirical models of the magnetospheric magnetic field, Rev. Geophys., 32, 139-157

Joshi, M. M., Haberle, R. M., Reynolds, R. T., 1997, Simulations of the atmospheres of synchronously rotating terrestrial planets orbiting $\mathrm{M}$ dwarfs: Conditions for atmospheric collapse and the implications for habitability, Icarus, 129, 450-465

Joy, S. P., Kivelson, M. G., Walker, R. J., Khurana, K. K., Russell, C. T., Ogino, T., 2002, Probabilistic models of the Jovian magnetopause and bow shock locations, J. Geophys. Res., 107, SMP 17-1-17-17

Kai, K., Melrose, D. B., Suzuki, S., 1985, Storms, in Solar Radiophysics, (Eds.) D. J. McLean, N. R. Labrum, chap. 16, pp. 415-441, Cambridge University Press, Cambridge

Kallenrode, M.-B., 2000, Galactic cosmic rays, in The Outer Heliosphere: Beyond the Planets, (Eds.) K. Scherer, H. Fichtner, E. Marsch, chap. 7, pp. 165-190, Copernicus Ges., Katlenburg-Lindau

Kassim, N. E., Lazio, T. J. W., Ray, P. S., Crane, P. C., Hicks, B. C., Stewart, K. P., Cohen, A. S., Lane, W. M., 2004, The low frequency array (LOFAR): opening a new window on the universe, Planet. Space Sci., 52, 1343-1349

Kasting, J. F., 1997, Habitable zones around low mass stars and the search for extraterrestrial life, Origins Life Evol. Biosphere, 27, 291-307

Kasting, J. F., Whitmire, D. P., Reynolds, R. T., 1993, Habitable zones around main sequence stars, Icarus, 101, 108-128

Kertz, W., 1995, Einführung in die Geophysik, vol. II, Spektrum Akademischer Verlag, Heidelberg

Khodachenko, M., Ribas, I., Lammer, H., Grießmeier, J.-M., Leitner, M., Selsis, F., Eiroa, C., Hanslmeier, A., Biernat, H. K., Farrugia, C. J., Rucker, H. O., 2006, CME activity of low mass $\mathrm{M}$ stars as an important factor for the habitability of terrestrial exoplanets. Part I: CME impact on expected magnetospheres of Earth-like exoplanets in close-in habitable zones, Astrobiology, submitted

Konacki, M., Torres, G., Jha, S., Sasselov, D. D., 2003a, An extrasolar planet that transits the disk of its parent star, Nature, 421, 507-509 
Konacki, M., Torres, G., Sasselov, D. D., Jha, S., 2003b, High-resolution spectroscopic follow-up of OGLE planetary transit candidates in the galactic bulge: Two possible Jupiter-mass planets and two blends, Astrophys. J., 597, 1076-1091

Konacki, M., Torres, G., Sasselov, D. D., Pietrzyński, G., Udalski, A., Jha, S., Ruiz, M. T., Gieren, W., Minniti, D., 2004, The transiting extrasolar giant planet around the star OGLE-TR-113, Astrophys. J., 609, L37-L40

Konacki, M., Torres, G., Sasselov, D. D., Jha, S., 2005, A transiting extrasolar giant planet around the star OGLE-TR-10, Astrophys. J., 624, 372-377

Kuznetsov, K. V., Nymmik, R. A., 2002, The dependence of solar energetic particle fluxes in the Earth-Mars-Earth route on solar activity period, Adv. Space Res., 30, 981-984

Lammer, H., Selsis, F., Ribas, I., Guinan, E. F., Bauer, S. J., Weiss, W. W., 2003, Atmospheric loss of exoplanets resulting from stellar X-ray and extreme-ultraviolet heating, Astrophys. J., 598, L121-L124

Lammer, H., Ribas, I., Grießmeier, J.-M., Penz, T., Hanslmeier, A., Biernat, H. K., 2004, A brief history of the solar radiation and particle flux evolution, Hvar Obs. Bull., 28, $139-155$

Lammer, H., Lichtenegger, H. I. M., Kulikov, Y. N., Grießmeier, J.-M., Terada, N., Erkaev, N. V., Biernat, H. K., Khodachenko, M. L., Ribas, I., Penz, T., Selsis, F., 2006, CME activity of low mass $M$ stars as an important factor for the habitability of terrestrial exoplanets. Part II: CME induced ion pick up of Earth-like exoplanets in close-in habitable zones, Astrobiology, submitted

Lanzerotti, L. J., Krimigis, S. M., 1985, Comparative magnetospheres, Physics Today, 38, 24-37

Lara, A., González-Esparza, J. A., Gopalswamy, N., 2004, Characteristics of coronal mass ejections in the near Sun interplanetary space, Geofísica Internacional, 43, 75-82

Laskar, J., Correia, A. C. M., 2004, The rotation of extra-solar planets, in Extrasolar Planets: Today and Tomorrow, (Eds.) J.-P. Beaulieu, A. Lecavelier des Etangs, C. Terquem, vol. 321 of ASP Conference Series, pp. 401-410

Laskar, J., Robutel, P., 1993, The chaotic obliquity of the planets, Nature, 361, 608-612

Latham, D. W., Mazeh, T., Stefanik, R. P., Mayor, M., Burki, G., 1989, The unseen companion of HD114762: a probable brown dwarf, Nature, 339, 38-40

Lazio, T. J. W., Farrell, W. M., Dietrick, J., Greenless, E., Hogan, E., Jones, C., Hennig, L. A., 2004, The radiometric Bode's law and extrasolar planets, Astrophys. J., 612, $511-518$

Lecavelier des Etangs, A., Vidal-Madjar, A., McConnell, J. C., Hébrard, G., 2004, Atmospheric escape from hot Jupiters, Astron. Astrophys., 418, L1-L4 
Léger, A., Selsis, F., Sotin, C., Guillot, T., Despois, D., Mawet, D., Ollivier, M., Labèque, F. A., Brachet, C. V., Chazelas, B., Lammer, H., 2004, A new family of planetes? "Ocean-Planets", Icarus, 169, 499-504

Leigh, C., Collier Cameron, A., Horne, K., Penny, A., James, D., 2003, A new upper limit on the reflected starlight from $\tau$ Bootis b, Mon. Not. R. Astron. Soc., 344, 1271-1282

Lissauer, J. J., 2002, Extrasolar planets, Nature, 419, 355-358

Lubow, S. H., Tout, C. A., Livio, M., 1997, Resonant tides in close orbiting planets, Astrophys. J., 484, 866-870

MacDonald, G. J. F., 1964, Tidal friction, Rev. Geophys., 2, 467-541

Majid, W., Winterhalter, D., Chandra, I., Kuiper, T., Lazio, J., Naudet, C., Zarka, P., 2006, Search for radio emission from extrasolar planets: Preliminary analysis of GMRT data, in Planetary Radio Emissions VI, (Eds.) H. O. Rucker, W. S. Kurth, G. Mann, pp. 589-594, Austrian Academy of Sciences Press, Vienna

Mann, G., Jansen, F., MacDowall, R. J., Kaiser, M. L., Stone, R. G., 1999, A heliospheric model and type III radio bursts, Astron. Astrophys., 348, 614-620

Mao, S., Paczyński, B., 1991, Gravitational microlensing by double stars and planetary systems, Astrophys. J., 374, L37-L40

Marcy, G. W., Butler, R. P., Williams, E., Bildsten, L., Graham, J. R., Ghez, A. M., Jernigan, J. G., 1997, The planet aroung 51 Pegasi, Astrophys. J., 481, 926-935

Mariani, F., Neubauer, F. M., 1990, The interplanetary magnetic field, in Physics of the Inner Heliosphere, (Eds.) R. Schwenn, E. Marsch, vol. 1, chap. 4, pp. 183-206, SpringerVerlag, Berlin

Marsch, E., 1991, Kinetic physics of the solar wind plasma, in Physics of the Inner Heliosphere, (Eds.) R. Schwenn, E. Marsch, vol. 2, chap. 8, pp. 45-133, Springer-Verlag, Berlin

Mayor, M., Queloz, D., 1995, A Jupiter-mass companion to a solar-type star, Nature, 378, 355-359

Mazeh, T., Naef, D., Torres, G., Latham, D. W., Mayor, M., Beuzit, J.-L., Brown, T. M., Buchhave, L., Burnet, M., Carney, B. W., Charbonneau, D., Druiker, G. A., Laird, J. B., Pepe, F., Perrier, C., Queloz, D., Santos, N. C., Sivan, J.-P., Udry, S., Zucker, S., 2000, The spectroscopic orbit of the planetary companion transiting HD 209458, Astrophys. J., 532, L55-L58

McKibben, R. B., 2001, Cosmic rays at all latitudes in the inner heliosphere, in The Heliosphere Near Solar Minimum, (Eds.) A. Balogh, R. G. Marsden, E. J. Smith, chap. 8, pp. 327-371, Springer, London 
Mel'nik, V. N., Konovalenko, A. A., Rucker, H. O., Stanislavsky, A. A., Abranin, E. P., Lecacheux, A., Mann, G., Warmuth, A., Zaitsev, V. V., Boudjada, M. Y., Dorovskii, V. V., Zaharenko, V. V., Lisachenko, V. N., Rosolen, C., 2004, Observations of solar type II bursts at frequencies 10-30 MHz, Solar Phys., 222, 151-166

Melo, C., Bouchy, F., Pont, F., Santos, N. C., Mayor, M., Queloz, D., Udry, S., 2004, Two new very hot Jupiters in the FLAMES spotlight, The ESO Messenger, 116, 32-34

Melrose, D. B., 1985, Plasma emission mechanisms, in Solar Radiophysics, (Eds.) D. J. McLean, N. R. Labrum, chap. 8, pp. 177-210, Cambridge University Press, Cambridge

Meyer, P., Ramaty, R., Webber, W. R., 1974, Cosmic rays - astronomy with energetic particles, Physics Today, 27, 23-32

Mignard, F., 2005, Overall science goals of the Gaia mission, in The Three-Dimensional Universe with Gaia, vol. SP-576 of ESA, pp. 5-14

Mizutani, H., Yamamoto, T., Fujimura, A., 1992, A new scaling law of the planetary magnetic fields, Adv. Space Res., 12, 265-279

Moutou, C., Coustenis, A., Schneider, J., Queloz, D., Mayor, M., 2003, Searching for helium in the exosphere of HD 209458b, Astron. Astrophys., 405, 341-348

Moutou, C., Pont, F., Bouchy, F., Mayor, M., 2004, Accurate radius and mass of the transiting exoplanet OGLE-TR-132b, Astron. Astrophys., 424, L31-L34

Murray, C. D., Dermott, S. F., 1999, Solar System Dynamics, Cambridge University Press, Cambridge

Narita, N., Suto, Y., Winn, J. N., Turner, E. L., Aoki, W., Leigh, C. J., Sato, B., Tamura, M., Yamada, T., 2005, Subaru HDS transmission spectroscopy of the transiting extrasolar planet HD 209458b, Publ. Astron. Soc. Japan, 57, 471-480

Nellis, W. J., 2000, Metallization of fluid hydrogen at $140 \mathrm{GPa}$ (1.4 Mbar): implications for Jupiter, Planet. Space Sci., 48, 671-677

Nelson, G. J., Sheridan, K. V., Suzuki, S., 1985, Measurement of solar flux density and polarization, in Solar Radiophysics, (Eds.) D. J. McLean, N. R. Labrum, chap. 6, pp. 113-154, Cambridge University Press, Cambridge

Neubauer, F. M., 1978, Possible strengths of dynamo magnetic fields of the Galilean satellites and of Titan, Geophys. Res. Lett., 5, 905-908

Newkirk, Jr., G., 1980, Solar variability on time scales of $10^{5}$ years to $10^{9.6}$ years, in The Ancient Sun: Fossil Record in the Earth, Moon and Meteorites, (Eds.) R. O. Pepin, J. A. Eddy, R. B. Merrill, pp. 293-320

Ogilvie, G. I., Lin, D. N. C., 2004, Tidal dissipation in rotating giant planets, Astrophys. J., 610, 477-509 
Parker, E. N., 1958, Dynamics of the interplanetary gas and magnetic fields, Astrophys. J., 128, 664-676

Pätzold, M., Rauer, H., 2002, Where are the massive close-in extrasolar planets?, Astrophys. J., 568, L117-L120

Pätzold, M., Carone, L., Rauer, H., 2004, Tidal interactions of close-in extrasolar planets: the OGLE cases, Astron. Astrophys., 427, 1075-1080

Peale, S. J., 1999, Origin and evolution of the natural satellites, Ann. Rev. Astron. Astrophys., 37, 533-602

Perryman, M., Heinaut, O., 2005, Report by the ESA-ESO working group on extrasolar planets, Tech. Rep. 1, ESA-ESO Working Groups

Perryman, M. A. C., 2000, Extra-solar planets, Rep. Prog. Phys., 63, 1209-1272

Petrinec, S. M., Russell, C. T., 1997, Hydrodynamic and MHD equations across the bow shock and along the surfaces of planetary obstacles, Space Sci. Rev., 79, 757-791

Pont, F., Bouchy, F., Queloz, D., Santos, N. C., Melo, C., Mayor, M., Udry, S., 2004, The "missing link": a 4-day period transiting exoplanet around OGLE-TR-111, Astron. Astrophys., 426, L15-L18

Pont, F., Melo, C. H. F., Bouchy, F., Udry, S., Queloz, D., Mayor, M., Santos, N. C., 2005, A planet-sized transiting star around OGLE-TR-122. Accurate mass and radius near the hydrogen-burning limit, Astron. Astrophys., 433, L21-L24

Prangé, R., Zarka, P., Ballester, G. E., Livengood, T. A., Denis, L., Carr, T., Reyes, F., Bame, S. J., Moos, H. W., 1993, Correlated variations of UV and radio emissions during an outstanding Jovian auroral event, J. Geophys. Res., 98, 18 779-18 791

Preusse, S., 2006, Szenarien der Plasmawechselwirkung in kurzperiodischen extrasolaren Planetensystemen, Ph.D. thesis, Technische Universität Braunschweig, ISBN 3936586-48-9, Copernicus-GmbH Katlenburg-Lindau

Preusse, S., Kopp, A., Büchner, J., Motschmann, U., 2005, Stellar wind regimes of closein extrasolar planets, Astron. Astrophys., 434, 1191-1200

Prölss, G. W., 2001, Physik des erdnahen Weltraums, Springer-Verlag, Berlin

Raith, W. (Ed.), 1997, Erde und Planeten, vol. 7 of Bergmann Schäfer Lehrbuch der Experimentalphysik, Walter de Gruyter, Berlin

Reeves, G. D., Cayton, T. E., Gary, S. P., Belian, R. D., 1992, The great solar energetic particle events of 1989 observed from geosynchronous orbit, J. Geophys. Res., 97, 6219-6226

Richardson, L. J., Deming, D., Wiedemann, G., Goukenleuque, C., Steyert, D., Harrington, J., Esposito, L. W., 2003, Infrared observations during the secondary eclipse of HD 209458b. I. 3.6 micron occultation spectroscopy using the very large telescope, Astrophys. J., 584, 1053-1062 
Rivera, E. J., Lissauer, J. J., Butler, R. P., Marcy, G. W., Vogt, S. S., Fischer, D. A., Brown, T. M., Laughlin, G., Henry, G. W., 2005, A $~ 7.5 M_{\oplus}$ planet orbiting the nearby star, GJ 876, Astrophys. J., 634, 625-640

Roberts, P. H., Glatzmaier, G. A., 2000, Geodynamo theory and simulations, Rev. Mod. Phys., 72, 1081-1123

Rosenblatt, F., 1971, A two-color photometric method for detection of extra-solar planetary systems, Icarus, 14, 71-93

Rubenstein, E. P., Schaefer, B. E., 2000, Are superflares on solar analogues caused by extrasolar planets?, Astrophys. J., 529, 1031-1033

Rucker, H. O., 1987, Solar wind influence on non-thermal planetary radio emission, Ann. Geophys., 5A, 1-11

Rucker, H. O., 2002, Radioastronomical aspects in the search for extrasolar planets, ESA, SP-518, 421

Russell, C. T., 1978, Re-evaluating Bode's law of planetary magnetism, Nature, 272, $147-148$

Russell, C. T., 1979, Scaling law test and two predictions of planetary magnetic moments, Nature, 281, 552-553

Russell, C. T., 1993, Magnetic fields of the terrestrial planets, J. Geophys. Res., 98, $18681-18695$

Russell, C. T., Walker, R. J., 1995, The magnetospheres of the outer planets, in Introduction to space physics, (Eds.) M. G. Kivelson, C. T. Russell, chap. 15, pp. 503-520, Cambridge University Press, Cambridge

Ryabov, V. B., Zarka, P., Ryabov, B. P., 2004, Search of exoplanetary radio signals in the presence of strong interference: enhancing sensitivity by data accumulation, Planet. Space Sci., 52, 1479-1491

Saar, S. H., Cuntz, M., 2001, A search for Ca II emission enhancement in stars resulting from nearby giant planets, Mon. Not. R. Astron. Soc., 325, 55-59

Saar, S. H., Cuntz, M., Shkolnik, E., 2004, Stellar activity enhancement by planets: Theory and observations, in Stars as Suns: Activity, Evolution, and Planets, (Eds.) A. K. Dupree, A. O. Benz, vol. 219 of IAU Symposium, pp. 355-366

Saffe, C., Gómez, M., Chavero, C., 2005, On the ages of exoplanet host stars, Astron. Astrophys., 443, 609-626

Sánchez-Lavega, A., 2004, The magnetic field in giant extrasolar planets, Astrophys. J., 609, L87-L90

Sano, Y., 1993, The magnetic fields of the planets: A new scaling law of the dipole moments of the planetary magnetism, J. Geomag. Geoelectr., 45, 65-77 
Santos, N. C., Bouchy, F., Mayor, M., Pepe, F., Queloz, D., Udry, S., Lovis, C., Bazot, M., Benz, W., Bertaux, J.-L., Curto, G. L., Delfosse, X., Mordasini, C., Naef, D., Sivan, J.-P., Vauclair, S., 2004, The HARPS survey for southern extra-solar planets II. A 14 Earth-masses exoplanet around $\mu$ Arae, Astron. Astrophys., 426, L19-L23

Sasselov, D. D., 2003, The new transiting planet OGLE-TR-56b: Orbit and atmosphere, Astrophys. J., 596, 1327-1331

Sato, B., Fischer, D. A., Henry, G. W., Laughlin, G., Butler, R. P., Marcy, G. W., Vogt, S. S., Bodenheimer, P., Ida, S., Toyota, E., Wolf, A., Valenti, J. A., Boyd, L. J., Johnson, J. A., Wright, J. T., Ammons, M., Robinson, S., Strader, J., McCarthy, C., Tah, K. L., Minniti, D., 2005, The N2K consortium. II. A transiting hot Saturn around HD 149026 with a large dense core, Astrophys. J., 633, 465-473

Schaefer, B. E., King, J. R., Deliyannis, C. P., 2000, Superflares on ordinary solar-type stars, Astrophys. J., 529, 1026-1030

Scheffler, H., 1997, Sterne und interstellare Materie, in Sterne und Weltraum, (Ed.) W. Raith, vol. 8 of Bergmann Schäfer Lehrbuch der Experimentalphysik, chap. 2, pp. 59-171, Walter de Gruyter, Berlin

Scherer, K., Fichtner, H., Stawicki, O., 2002, Shielded by the wind: the influence of the interstellar medium on the environment of Earth, Journal of Atmospheric and SolarTerrestrial Physics, 64, 795-804

Schilling, G., 1996, 'Hot Jupiters' leave theorists in the cold, Science, 273, 429

Schwenn, R., 1990, Large-scale structure of the interplanetary medium, in Physics of the Inner Heliosphere, (Eds.) R. Schwenn, E. Marsch, vol. 1, chap. 3, pp. 99-181, Springer-Verlag, Berlin

Seager, S., Hui, L., 2002, Constraining the rotation rate of transiting extrasolar planets by oblateness measurements, Astrophys. J., 574, 1004-1010

Seager, S., Sasselov, D. D., 1998, Extrasolar giant planets under strong stellar irradiation, Astrophys. J., 502, L157-L161

Selsis, F., 2004, The atmosphere of terrestrial exoplanets: Detection and characterization, in ASP Conf. Ser. 321: Extrasolar Planets: Today and Tomorrow, (Eds.) J.-P. Beaulieu, A. Lecavelier des Etangs, C. Terquem, pp. 170-181

Selsis, F., Despois, D., Parisot, J.-P., 2002, Signature of life on exoplanets: Can Darwin produce false positive detections?, Astron. Astrophys., 388, 985-1003

Seo, E. S., McDonald, F. B., Lal, N., Webber, W. R., 1994, Study of cosmic-ray H and He isotopes at $23 \mathrm{AU}$, Astrophys. J., 432, 656-664

Shea, M. A., Smart, D. F., 2000, Cosmic ray implications for human health, Space Sci. Rev., 93, 187-205 
Sheridan, K. V., McLean, D. J., 1985, The quiet sun at metre wavelengths, in Solar Radiophysics, (Eds.) D. J. McLean, N. R. Labrum, chap. 17, pp. 443-466, Cambridge University Press, Cambridge

Shkolnik, E., Walker, G. A. H., Bohlender, D. A., 2003, Evidence for planet-induced chromospheric activity on HD 179949, Astrophys. J., 597, 1092-1096

Shkolnik, E., Walker, G. A. H., Bohlender, D. A., 2004, Erratum: "Evidence for planetinduced chromospheric activity on HD 179949”, Astrophys. J., 609, 1197

Shkolnik, E., Walker, G. A. H., Bohlender, D. A., Gu, P.-G., Kürster, M., 2005, Hot Jupiters and hot spots: The short- and long-term chromospheric activity on stars with giant plants, Astrophys. J., 622, 1075-1090

Showman, A. P., Guillot, T., 2002, Atmospheric circulation and tides of "51 Pegasus b-like" planets, Astron. Astrophys., 385, 166-180

Siscoe, G. L., 1979, Towards a comparative theory of magnetospheres, in Solar System Plasma Physics, (Eds.) C. F. Kennel, L. J. Lanzerotti, E. N. Parker, vol. II, chap. II.8, pp. 319-402, North-Holland Publishing Company

Smart, D. F., Shea, M. A., 2002, A review of solar proton events during the 22nd solar cycle, Adv. Space Res., 30, 1033-1044

Smart, D. F., Shea, M. A., Flückiger, E. O., 2000, Magnetospheric models and trajectory computations, Space Sci. Rev., 93, 305-333

Smoluchowski, R., 1967, Internal structure and energy emission of Jupiter, Nature, 215, 691-695

Smoluchowski, R., 1975, Jupiter's molecular hydrogen layer and the magnetic field, Astrophys. J., 200, L119-L121

Sozzetti, A., Yong, D., Torres, G., Charbonneau, D., Latham, D. W., Allende Prieto, C., Brown, T. M., Carney, B. W., Laird, J. B., 2004, High-resolution spectroscopy of the transiting planet host star TrES-1, Astrophys. J., 616, L167-L170

Stadelmann, A., 2005a, Globale Effekte einer Erdmagnetfeldumkehrung: Magnetosphärenstruktur und kosmische Teilchen, Ph.D. thesis, Technische Universität Braunschweig, ISBN 3-936586-42-X, Copernicus-GmbH Katlenburg-Lindau, URL: http://www.digibib.tu-bs.de/?docid=00000002

Stadelmann, A., 2005b, Energetic Particle Orbits in Magnetospheres (EPOM), Technische Universität Braunschweig, Version 1.1

Stern, D. P., 1985, Parabolic harmonics in magnetospheric modeling: The main dipole and the ring current, J. Geophys. Res., 90, 10 851-10 863

Stevens, I. R., 2005, Magnetospheric radio emission from extrasolar giant planets: the role of the host stars, Mon. Not. R. Astron. Soc., 356, 1053-1063 
Stevenson, D. J., 1979, Turbulent thermal convection in the presence of rotation and a magnetic field: A heuristic theory, Geophys. Astrophys. Fluid Dynamics, 12, 139-169

Stevenson, D. J., 1982, Interiors of the giant planets, Annu. Rev. Earth. Planet. Sci., 10, 257-295

Stevenson, D. J., 1983, Planetary magnetic fields, Rep. Prog. Phys., 46, 555-620

Stevenson, D. J., 2003, Planetary magnetic fields, Earth Planet. Sci. Lett., 208, 1-11

Stevenson, D. J., Spohn, T., Schubert, G., 1983, Magnetism and thermal evolution of the terrestrial planets, Icarus, 54, 466-489

Stix, M., 1977, Planetarische Dynamos, J. Geophys., 43, 695-717

Stracke, B., 2004, Wechselwirkung von Sternenwinden mit extrasolaren Planeten, Diplomarbeit, Universität zu Köln

Struve, O., 1952, Proposal for a project of high-precision stellar radial velocity work, Observatory, 72, 199-200

Torres, G., Konacki, M., Sasselov, D. D., Jha, S., 2004, New data and improved parameters for the extrasolar transiting planet OGLE-TR-56b, Astrophys. J., 609, 1071-1075

Tout, C. A., Pols, O. R., Eggleton, P. P., Han, Z., 1996, Zero-age main-sequence radii and luminosities as analytic functions of mass and metallicity, Mon. Not. R. Astron. Soc., $281,257-262$

Trimble, V., 2004, The quest for other worlds, 350 BCE to 1995 CE, in AIP Conf. Proc. 713: The Search for Other Worlds, (Eds.) S. S. Holt, D. Deming, pp. 3-10, American Institute of Physics

Udry, S., Mayor, M., Santos, N. C., 2003, Statistical properties of exoplanets I. The period distribution: Constraints for the migration scenario, Astron. Astrophys., 407, 369-376

Vidal-Madjar, A., Lecavelier des Etangs, A., Désert, J.-M., Ballester, G. E., Ferlet, R., Hébrard, G., Mayor, M., 2003, An extended upper atmosphere around the extrasolar planet HD209458b, Nature, 422, 143-146

Vidal-Madjar, A., Désert, J.-M., Lecavelier des Etangs, A., Hébrard, G., Ballester, G. E., Ehrenreich, D., Ferlet, R., McConnell, J. C., Mayor, M., Parkinson, C. D., 2004, Detection of oxygen and carbon in the hydrodynamically escaping atmosphere of the extrasolar planet HD 209458b, Astrophys. J., 604, L69-L72

Vogt, J., Glassmeier, K.-H., 2001, Modelling the paleomagnetosphere: strategy and first results, Adv. Space Res., 28, 863-868

Voigt, G. H., 1979, Influence of the interplanetary magnetic field on the position of the dayside magnetopause, in Proceedings of Magnetospheric Boundary Layers Conference, Alpach, pp. 315-321, ESA SP-148 
Voigt, G.-H., 1981, A mathematical magnetospheric field model with independent physical parameters, Planet. Space Sci., 29, 1-20

Voigt, G.-H., 1995, Magnetospheric configuration, in Handbook of atmospheric electrodynamics, (Ed.) H. Volland, vol. II, chap. 11, pp. 333-388, CRC Press

Walker, R. J., Russell, C. T., 1995, Solar-wind interactions with magnetized planets, in Introduction to space physics, (Eds.) M. G. Kivelson, C. T. Russell, chap. 6, pp. 164182, Cambridge University Press, Cambridge

Warmuth, A., Mann, G., 2005, The application of radio diagnostics to the study of the solar drivers of space weather, in Space Weather: The physics behind the slogan, vol. 656 of Lecture Notes in Physics, pp. 49-70, Springer

Weber, E. J., Davis, Jr., L., 1967, The angular momentum of the solar wind, Astrophys. J., 148, 217-227

White, S. M., Kundu, M. R., Jackson, P. D., 1989, Simple non-thermal models for the quiescent radio emission of dMe flare stars, Astrophys. J., 225, 112-124

Williams, D. M., Kasting, J. F., 1997, Habitable planets with high obliquities, Icarus, 129, 254-267

Winglee, R. M., Dulk, G. A., Bastian, T. S., 1986, A search for cyclotron Maser radiation from substellar and planet-like companions of nearby stars, Astrophys. J., 209, L59L62

Wolf, R. A., 1995, Magnetospheric configuration, in Introduction to space physics, (Eds.) M. G. Kivelson, C. T. Russell, chap. 10, pp. 288-329, Cambridge University Press, Cambridge

Wolszczan, A., Frail, D. A., 1992, A planetary system around the millisecond pulsar PSR1257+12, Nature, 355, 145-147

Wood, B. E., 2004, Astrospheres and solar-like stellar winds, Living Rev. Solar Phys., 1, 2, URL: http://www.livingreviews.org/lrsp-2004-2, accessed on 11 October 2005

Wood, B. E., Müller, H.-R., Zank, G. P., Linsky, J. L., 2002, Measured mass-loss rates of solar-like stars as a function of age and activity, Astrophys. J., 574, 412-425

Wood, B. E., Müller, H.-R., Zank, G. P., Linsky, J. L., Redfield, S., 2005a, New mass-loss measurements from astrospheric Ly $\alpha$ absorption, Astrophys. J., 628, L143-L146

Wood, B. E., Redfield, S., Linsky, J. L., Müller, H.-R., Zank, G. P., 2005b, Stellar Ly $\alpha$ emission lines in the Hubble Space Telecope archive: Intrinsic line fluxes and absorption from the heliosphere and astrospheres, Astrophys. J. Suppl. Ser., 159, 118-140

Wu, C. S., Lee, L. C., 1979, A theory of the terrestrial kilometric radiation, Astrophys. J., $230,621-626$ 
Yantis, W. F., Sullivan, III., W. T., Erickson, W. C., 1977, A search for extra-solar jovian planets by radio techniques, Bull. Am. Astron. Soc., 9, 453

Zarka, P., 1992, The auroral radio emissions from planetary magnetospheres: What do we know, what don't we know, what do we learn from them?, Adv. Space Res., 12, (8)99-(8)115

Zarka, P., 1998, Auroral radio emissions at the outer planets: Observations and theories, J. Geophys. Res., 103, 20 159-20 194

Zarka, P., 2004, Non-thermal radio emissions from extrasolar planets, in Extrasolar planets: Today and Tomorrow, (Eds.) J.-P. Beaulieu, A. Lecavelier des Etangs, C. Terquem, vol. 321 of ASP Conference Series, pp. 160-169

Zarka, P., Pedersen, B. M., Lecacheux, A., Kaiser, M. L., Desch, M. D., Farrell, W. M., Kurth, W. S., 1995, Radio emissions from Neptune, in Neptune and Triton, (Ed.) D. P. Cruikshank, pp. 341-387, Univ. of Ariz. Press, Tuscon

Zarka, P., Queinnec, J., Ryabov, B. P., Ryabov, V. B., Shevchenko, V. A., Arkhipov, A. V., Rucker, H. O., Denis, L., Gerbault, A., Dierich, P., Rosolen, C., 1997, Groundbased high sensitivity radio astronomy at decameter wavelengths, in Planetary Radio Emissions IV, (Eds.) H. O. Rucker, S. J. Bauer, A. Lecacheux, pp. 101-127, Austrian Academy of Sciences Press, Vienna

Zarka, P., Treumann, R. A., Ryabov, B. P., Ryabov, V. B., 2001, Magnetically-driven planetary radio emissions and application to extrasolar planets, Astrophys. Space Sci., 277, 293-300

Zarka, P., Cecconi, B., Kurth, W. S., 2004, Jupiter's low frequency radio spectrum from Cassini/Radio and Plasma Wave Science (RPWS) absolute flux density measurements, J. Geophys. Res., 109, A09S15

Zaroubi, S., Silk, J., 2005, LOFAR as a probe of the sources of cosmological reionization, Mon. Not. R. Astron. Soc., 360, L64-L67

Zhang, M. H. G., Luhmann, J. G., Bougher, S. W., Nagy, A. F., 1993, The ancient oxygen exosphere of Mars: Implications for atmosphere evolution, J. Geophys. Res., 98, $10915-10923$

Zuchowski, L., 2005, Investigation of Exomagnetospheres by Use of Numerical Simulations, Bachelor thesis, International University Bremen 


\title{
Acknowledgements
}

\author{
Anar kaluva tielyanna
}

J. R. R. Tolkien

It is my duty and pleasure to acknowledge advice and support I received during the work on this thesis.

First of all, I would like to thank Prof. Dr. U. Motschmann for suggesting the fascinating subject of extrasolar planets, for his support during the last years, and for giving me the opportunity to present my results on various conferences.

From the beginning, Prof. Dr. K.-H. Glassmeier followed the progress of this work with interest. I would also like to thank him for his support.

I had the privilege to enjoy the hospitality of the Institute for Space Research of the Austrian Academy of Sciences in Graz. For this, for his continued interest, and for taking over the task of reviewing this thesis, I would like to thank Prof. Dr. H. O. Rucker.

As a member of the International Max Planck Research School on Physical Processes in the Solar System and Beyond I had the opportunity to obtain insight into different subjects within the field of astrophysics, which I gratefully acknowledge. I would also like to thank the coordinator of the research school, Dr. Dieter Schmitt.

There are a number of scientific collaborations from which this project has strongly benefitted. I would like to thank Prof. Dr. G. Mann for giving me an introduction to solar radio emission, Dr. Helmut Lammer for our cooperation with respect to atmospheric escape and planetary habitability, Dr. Maxim Khodachenko for our collaboration on the subject of stellar coronal mass ejections, Dr. Franck Selsis for discussions about habitable zones, Anja Stadelmann for the fruitful cooperation concerning galactic cosmic rays, and Sabine Preusse for many discussions on stellar winds and on planetary magnetospheres.

I would also like to thank the many colleagues who were responsible for a pleasant working atmosphere at the Technical University of Braunschweig: Thorsten Bagdonat, Ingo von Borstel, Alexander Bößwetter, Andreas Friedrich, Simon Grossjohann, Verena Grützun, Matthias Grzeschik, Andreas Honecker, Fabian Heidrich-Meisner, Joachim Müller, Matthias Neef, Marcus Renner, Michael Rost, Anja Stadelmann, and Sven Simon. Special thanks go to Thorsten Badonat and Marcus Renner, who taught me how to administrate a computer network of Linux PCs and to Renate Strassek, who helped me with the administrative duties.

I am very grateful to Anja Stadelmann, Thorsten Bagdonat, Sabine Preusse, Jan-Oliver Kliemann, Barbara Stracke, Gerhart Grießmeier and Jérôme Grießmeier for thousands of valuable suggestions made during the stage of proof-reading.

Finally, I would like to thank all my friends and my family. One way or another, they all supported me throughout this work, and I would like to thank all of them. 